\title{
Redox regulation in pulmonary fibrosis
}

Citation for published version (APA):

Veith, C. (2019). Redox regulation in pulmonary fibrosis: towards therapeutic targets. [Doctoral Thesis, Maastricht University]. Gildeprint Drukkerijen. https://doi.org/10.26481/dis.20190524cv

Document status and date:

Published: 01/01/2019

DOI:

$10.26481 /$ dis.20190524cv

Document Version:

Publisher's PDF, also known as Version of record

\section{Please check the document version of this publication:}

- A submitted manuscript is the version of the article upon submission and before peer-review. There can be important differences between the submitted version and the official published version of record.

People interested in the research are advised to contact the author for the final version of the publication, or visit the DOI to the publisher's website.

- The final author version and the galley proof are versions of the publication after peer review.

- The final published version features the final layout of the paper including the volume, issue and page numbers.

Link to publication

\footnotetext{
General rights rights.

- You may freely distribute the URL identifying the publication in the public portal. please follow below link for the End User Agreement:

www.umlib.nl/taverne-license

Take down policy

If you believe that this document breaches copyright please contact us at:

repository@maastrichtuniversity.nl

providing details and we will investigate your claim.
}

Copyright and moral rights for the publications made accessible in the public portal are retained by the authors and/or other copyright owners and it is a condition of accessing publications that users recognise and abide by the legal requirements associated with these

- Users may download and print one copy of any publication from the public portal for the purpose of private study or research.

- You may not further distribute the material or use it for any profit-making activity or commercial gain

If the publication is distributed under the terms of Article $25 \mathrm{fa}$ of the Dutch Copyright Act, indicated by the "Taverne" license above, 


\section{Redox regulation in pulmonary fibrosis}

towards therapeutic targets

Carmen Veith 
The studies presented in this thesis were conducted within NUTRIM School of Nutrition and Translational Research in Metabolism of Maastricht University.
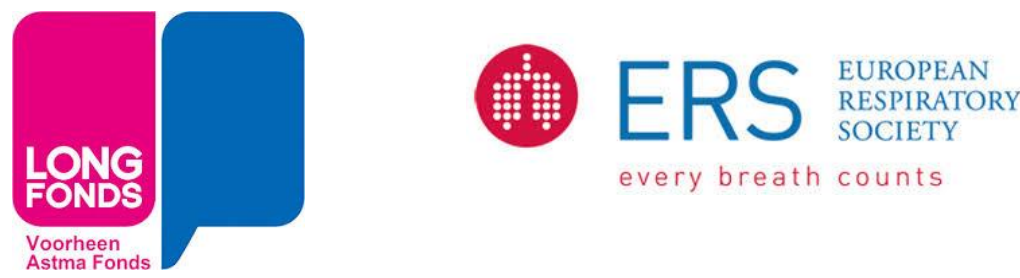

every breath counts

The work described in this thesis was financially supported by the NUTRIM graduate program, the Kootstra Talent Fellowship, the Longfonds (9.2.17.214FE) and the European Respiratory Society (STRTF201804-00377) granted to C. Veith.

(c) Carmen Veith, 2019

Redox regulation in pulmonary fibrosis: towards therapeutic targets

Layout: $\quad$ Carmen Veith

Cover design: Carmen Veith, Birgit Hettler

Printed by: Gildeprint, Enschede

ISBN:

978-94-6323-636-2

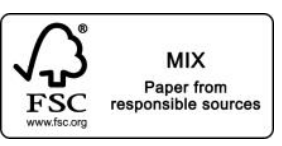

All rights are reserved. For articles published or accepted for publication, the copyright has been transferred to the respective publisher. No parts of this thesis may be reproduced, stored in a retrieval system or transmitted in any form or by any means, without prior permission from the author, or, when appropriate, from the publisher of the publication. 


\title{
Redox regulation in pulmonary fibrosis
}

\author{
towards therapeutic targets
}

\section{Dissertation}

To obtain the degree of Doctor at Maastricht University

on the authority of the Rector Magnificus, Prof. Dr. Rianne M. Letschert

in accordance with the decision of the board of Deans to be defended in public on Friday, the $24^{\text {th }}$ of May 2019 at 10.00 hours

by

\section{Carmen Veith}

Born on February $3^{\text {rd }} 1990$

in Bühl, Germany 


\section{Promotors}

Prof. Dr. Frederik-Jan van Schooten

Prof. Dr. Albert van der Vliet (University of Vermont, USA)

\section{Co-promotor}

Dr. Agnes W. Boots

\section{Assessment Committee}

Prof. Dr. Annemie Schols (voorzitter)

Dr. Matthijs Blankesteijn

Dr. Wilfred Germeraad

Prof. Dr. Jan Grutters (Utrecht University)

Prof. Dr. Pieter Hiemstra (Leiden University) 
Für Mama und Papa 



\section{Table of contents}

Chapter 1 General introduction and aim of the thesis

Chapter 2 Redox imbalance in IPF: a role for oxidant crosstalk between

NOX enzymes and mitochondria

Chapter 3 The redox balance in pulmonary fibrosis is modulated by the plant flavonoid quercetin

Chapter 4 The dietary antioxidant quercetin reduces hallmarks of bleomycin-induced fibrogenesis in mice

Chapter 5 Profibrotic signaling by TGF- $\beta 1$ involves NADPH oxidase 4 dependent activation of the tyrosine kinase FYN

Chapter 6 SRC family kinases mediates TGF- $\beta 1$ induced

myofibroblast activation

Chapter 7 Inhibition of SRC family kinases alleviates impaired expression

levels of key regulators of mitochondrial biogenesis and mitophagy in bronchial epithelial cells of IPF patients

Chapter 8 Differences in treatment response in bronchial epithelial cells from Idiopathic Pulmonary Fibrosis patients: a first step towards personalized medicine?

Chapter 9 Summary and Discussion

Addendum Samenvatting

Zusammenfassung

Valorization

Acknowledgements

271

List of publications

275

Curriculum Vitae 



\section{Chapter 1}

General introduction and outline of the thesis 


\section{Summary}

The lungs are continuously exposed to high concentrations of oxygen, which makes them especially vulnerable to oxidative damage. In addition to exogenous sources of oxidants, various lung cell types can also generate reactive oxygen species (ROS) themselves, either as byproducts of cellular metabolism or by enzyme systems such as the NADPH oxidases (NOX). ROS have various physiological functions in host defense, immune regulation, cell proliferation and differentiation but can also be harmful when produced in excessive amounts. To effectively regulate the biological actions of exogenous and endogenous ROS, various non-enzymatic and enzymatic antioxidants defense systems are present in lung cells to provide protection and to regulate redox-sensitive cell signaling. Chronic lung diseases such as idiopathic pulmonary fibrosis (IPF) are associated with an increased oxidant burden and IPF patients display various markers of oxidative stress such as oxidation of DNA and proteins. At the same time, IPF is also characterized by an increased expression of NOX4 and mitochondrial dysfunction as well as decreased antioxidant systems, which all further contribute to a disturbed antioxidant balance. Intriguingly, antioxidant treatment strategies have not been fully effective in the clinic so far. Recent studies have identified that SRC family kinases (SFK) contribute to the development of IPF and their activity can be regulated through redox-signaling. Consequently, inhibition of SFK has been suggested as treatment strategy in IPF. 


\section{The lungs}

The lungs represent the human body's largest interface with the external environment and are responsible for breathing. The lungs facilitate gas exchange by taking up oxygen from the atmosphere and transporting it into the bloodstream. Additionally, the lungs release carbon dioxide, a waste product from metabolism, from the bloodstream back into the atmosphere. The lungs are on either side of the chest and are part of the lower respiratory system that begins at the trachea and branches into the primary bronchi (Figure 1.1). The bronchi then divide into smaller branches, called bronchioles that eventually terminate in clusters of microscopic small air sacs called alveoli where the actual gas exchange takes place (1). The lungs contain approximately 2.400 kilometer of airways and 300-500 million alveoli providing an extremely large surface area of approximately $150 \mathrm{~m}^{2}$.
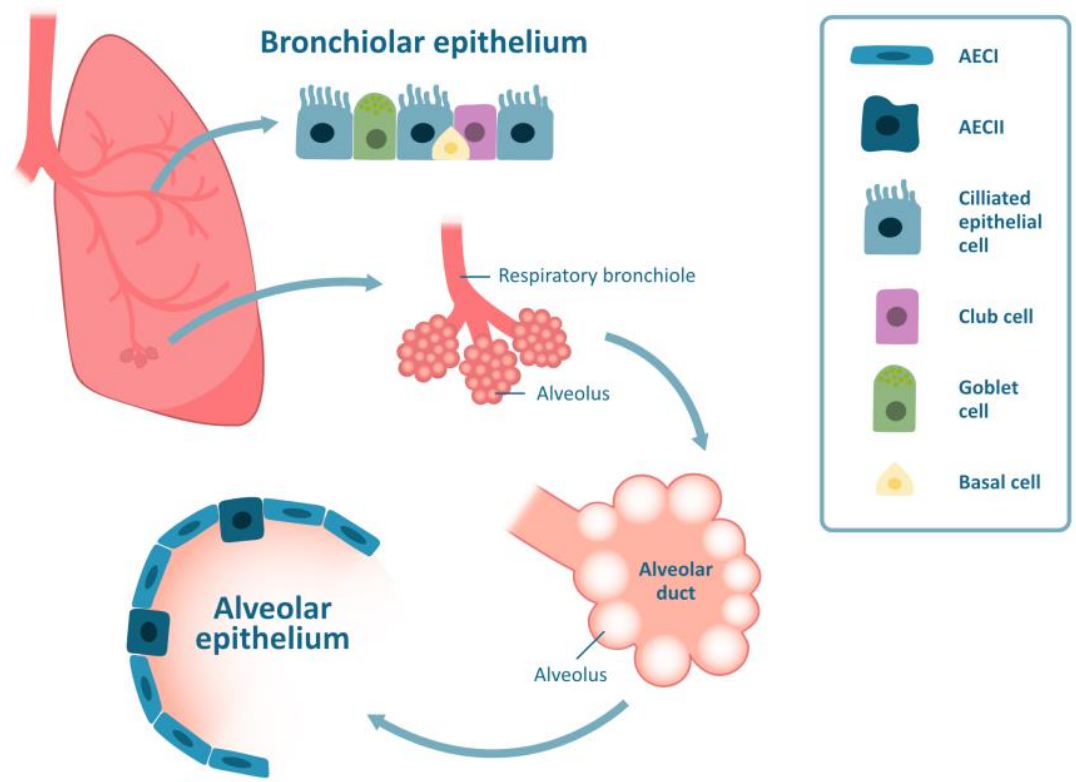

Figure 1.1: The anatomy of the lung. The trachea carries the air into the lungs and divides into airways, called bronchi. The bronchiolar epithelium consists of ciliated epithelial, club, goblet and basal cells. The bronchi divide into smaller airways that terminate in alveoli which is lined with the alveolar epithelium consisting of alveolar epithelial type I and II cells (AECI and AEC II).

Because of their direct contact with the outside work, the lungs are vulnerable to airborne pathogens and pollutants. Many commonly encountered environmental pollutants such as tobacco smoke, particulate matter and smog are thought to cause injury to the lungs via toxic chemicals and oxidant production. The respiratory tract is therefore equipped with detoxifying antimicrobial and antioxidant defense systems to minimize the risk of 
infection and injury while maintaining proper lung functions. The lower respiratory tract including the trachea, bronchi and bronchioles, is lined with the respiratory epithelium that forms the first line of defense preventing the entry of inhaled chemicals and pathogens. It provides a physical and mechanical barrier to impede the entry of airborne invaders, including bacteria and particles, and is also capable of initiating an immune response (2).

The cellular composition of the pulmonary epithelium changes along the proximal-todistal axis to fulfill different regional functions. The epithelium lining the bronchi and bronchioles consists of ciliated epithelial cells next to goblet cells, club cells and basal cells. In a healthy lung, ciliated epithelial cells have hair-like projections that coordinate the removal of pathogens to clear inhaled particles and pathogens from the tracheobronchial epithelium. These inhaled particles and pathogens are absorbed by mucus which is secreted by goblet cells and afterwards swept away by ciliated epithelial cells to be removed from the airways (3). Club cells protect the bronchiolar epithelium through the secretion of various proteins, glycoproteins and lipids which provide chemical and physical protection against inhaled compounds (4). Basal cells are located beneath the surface epithelium and maintain pulmonary integrity by differentiating into other cell types in response to damage. In response to injury, basal progenitor cells get activated and subsequently differentiate into secretory (e.g. goblet and club cells) and ciliated epithelial cells (5).

The alveolar epithelium, where the gas exchange takes place, is mainly composed of alveolar epithelial type I (AECI) and type II (AECII) cells (6). AECl cells are squamous epithelial cells responsible for gas exchange and occupy about $95 \%$ of the alveolar surface area (7). The cuboidal secretory AECII cells are mainly responsible for surfactant secretion which enables the gas exchange by lowering the surface tension within the alveoli (1). In addition, AECIl cells have important functions in lung defense mechanisms by releasing cytokines and producing oxidants to respectively induce an inflammatory response and kill pathogens (8). AECII cells also initiate the restoration of the alveolar epithelium after injury by either promoting the proliferation of new AECII cells or by differentiating into $\mathrm{AECl}$ cells, thereby maintaining the integrity of the epithelium (9). Type I cells are not able to differentiate and thus rely on differentiation from type II cells.

The lung epithelium in chronic lung diseases, such as pulmonary fibrosis, shows signatures of chronic injury, which results in an aberrant wound healing responses and remodeling of the lung tissue.

\section{Idiopathic pulmonary fibrosis - an overview}

Pulmonary fibrosis is a progressive interstitial lung disease in which the interstitium (walls between alveoli) becomes scarred, resulting in the destruction of the lung architecture. The lungs become stiffer and lose their elasticity causing symptoms such as non- 
productive dry cough and breathlessness, ultimately leading to death from respiratory failure. In many cases, the cause of the disease is not clear, leading to the diagnosis idiopathic pulmonary fibrosis (IPF). IPF is the most common form of idiopathic interstitial lung diseases, affecting approximately 5 million people worldwide with a median survival of only 2-4 years after diagnosis (10). The clinical course of IPF is very heterogeneous; some patients have a slower progression while the remainder have a faster progression with a rapid loss in lung function associated with shorter survival (Figure 1.2).

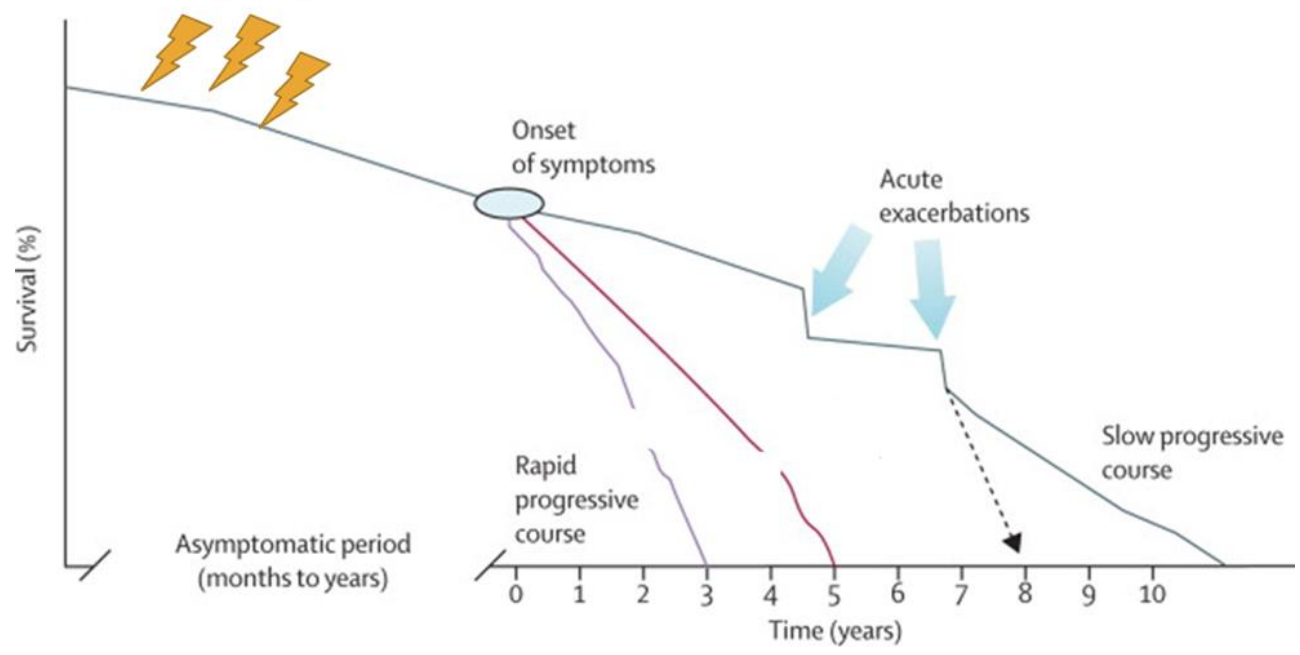

Figure 1.2: Clinical course of patients with IPF. The disease develops asymptomatically for months to years. Most patients have a slow progressive course of the disease, whereas other patients have acute exacerbations of the disease. A few patients have a rapid progressive course resulting in decreased survival. Figure adapted from (11).

Two thirds of IPF patients are older than 60 years at time of diagnosis (12). The prevalence of IPF is rising with increased age and estimated to be 227 per 100.000 in people older than 75 years, whereas only 4 per 100.000 people under 35 are affected (12). As the average life expectancy continues to increase worldwide, the incidence of age-related lung diseases such as IPF is also expected to increase at a rapid pace. Indeed, the overall incidence rate is already increasing every year $(13,14)$. The scarring of the lungs in IPF cannot be reversed indicating that there is no cure yet for IPF. Current treatments can slow down the progression but they cannot stop the disease.

IPF has several risk factors including aging (15) but other intrinsic/endogenous factors such as genomic instability, mitochondria dysfunction and altered intracellular signaling are also associated with IPF (16). Some people are also genetically predisposed to develop IPF due to genetic abnormalities in surfactant protein $C$ and $A 2$, genes involved in maintaining telomere length (TERT, TERC) as well as Mucin (Muc) 5B (17). An alteration in the Muc5B promoter sequence ( $r$ 35705950) has been identified as the strongest genetic risk factor for IPF and accounts for $30-35 \%$ of individuals developing IPF (18). Additionally, 
exposure to environmental compounds, e.g. cigarette smoke and asbestos, is another trigger for the development of IPF (19). Intriguingly, most of these compounds including cigarette smoke and asbestos also cause AEC apoptosis $(20,21)$ and promote mitochondrial dysfunction (22) as well as genomic instability (23).

\section{Disease mechanism}

During IPF manifestation, the lung homeostasis is disturbed resulting in a profibrotic milieu that affects both the survival and death of lung cells. IPS is characterized by impaired epithelial regeneration, accumulation of myofibroblasts producing collagen and chronic low-grade inflammation (Figure 1.3).

\section{Disease initiation: a key role for the lung epithelium}

It is generally accepted that the lung epithelium plays a crucial role in the initiation of the disease. Type II AECs proliferate and differentiate into type I AECs in response to injury and re-epithelialize the site where the barrier was broken. However, AECs in fibrotic repair are more prone to undergo apoptosis which is a prominent feature during IPF initiation (24). The lung epithelium of IPF patients shows a loss of cell integrity of the alveolar epithelium, illustrated by disruption of the basement membrane and collapse of the alveolar structure. Furhtermore, the number of $\mathrm{AECl}$ cells is reduced whereas hyperplasia of AECII cells develops, thus replacing type I AEC and causing ineffective reepithelialization. Additionally, senescence of epithelial cells occurs which results in a diminished regenerative capacity of AECs (25). Interestingly, epithelial cells can also undergo so-called epithelial-to-mesenchymal transition (EMT) which makes them lose their epithelial phenotype and acquire more mesenchymal/fibroblastic characteristics such as expression of $\alpha$-smooth muscle actin and fibronectin. EMT is considered to contribute to pulmonary fibrosis pathology by restructuring the lung due to the accumulation of fibroblasts which then differentiate into myofibroblasts, but its exact significance in IPF development is under debate (26).

\section{Disease progression: fibroblasts - the effector cells in IPF}

AECs secrete a variety of fibrotic mediators in response to injury, including plateletderived growth factor (PDGF), endothelin-1, angiotensin II, connective tissue growth factor (CTGF), and transforming-growth factor (TGF) $\beta$ which can induce differentiation of fibroblasts into myofibroblasts in vitro (27). Myofibroblasts are considered key effector cells in IPF as they actively participate in pulmonary remodeling by secreting extracellular matrix (ECM) proteins into the lung alveolar structure. During normal wound repair, myofibroblasts undergo apoptosis and clearance of the ECM occurs, whereas in IPF myofibroblasts are overactivated causing aberrant wound repair and excessive collagen deposition. Fibroblastic foci, an aggregation of myofibroblasts, are a hallmark of IPF (28) that correlates with decreased pulmonary function (29). Extracellular generation of $\mathrm{H}_{2} \mathrm{O}_{2}$ 
by lung myofibroblasts may mediate additional fibrogenic effects by inducing apoptosis of adjacent lung epithelial cells (30). Interestingly, fibroblasts from IPF patients have an apoptosis resistant phenotype and are associated with higher levels of senescence markers compared to fibroblasts from age-matched controls (31).

The role of inflammation in IPF progression

Although lungs from IPF patients display mild chronic inflammation (32), the precise role of inflammation in IPF is not clear yet. However, since immunosuppressants are not effective in treating the disease, inflammation has been suggested to play a secondary role in IPF progression (33), with a more specific role in rapid progressive IPF as well as in its exacerbations. Damaged epithelial cells release a variety of chemokines and cytokines including interleukin(IL)-8, tumor-necrosis factor (TNF)- $\alpha$ and interferon (IFN)- $\gamma$ that recruit inflammatory monocytes and neutrophils to the injured site. Neutrophils are part of the innate immune system and their recruitment and activation to the bronchoalveolar space have been shown to correlate with disease progression (34). Neutrophil recruitment is also suggested as a predictor of early mortality in IPF (35). Patients with rapid progressive IPF display an increased number of neutrophils and macrophages in their lungs (36) and the levels of the neutrophil chemoattractant, IL-8 is increased in IPF patients suffering from exacerbations compared to stable IPF patients (37). Macrophages are not only important in apoptotic cell clearance but they also participate in ECM remodeling through the secretion of matrix metalloproteinases (MMP) (38) as well as through ingestion of collagen (39).

Normally, monocytes differentiate into phagocytic macrophages that phagocytose dead cells and neutrophils. However, because of the continuous injury to the lung epithelium in IPF, neutrophils and macrophages are not eliminated quickly enough, thereby exacerbating the ongoing inflammation and fibrotic cycle (7). Additionally, alveolar neutrophils and macrophages undergo a respiratory burst upon phagocytosis leading to ROS production that also injures alveolar epithelial cells thereby promoting fibrosis (40). 


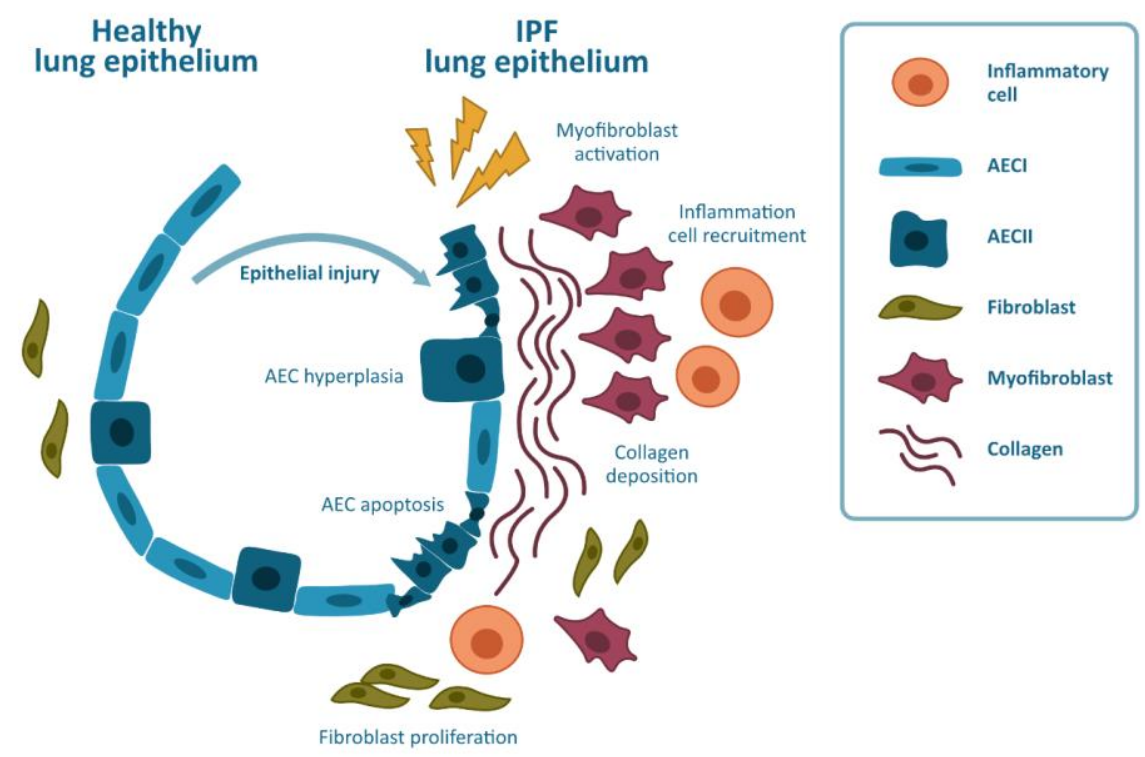

Figure 1.3: Summary of the pathologic features in IPF disease initiation and manifestation. A variety of genetic and environmental factors have been implicated as sources of injury to the lung and/or alveolar epithelium. Following epithelial injury, over-activation of repair pathways leads to AECII death, fibroblast recruitment and myofibroblast activation which causes excessive production of collagen and ECM deposition, and inflammatory cell recruitment.

\section{The role of TGF- $\beta 1$ in the pathogenesis of IPF}

The profibrotic cytokine transforming growth factors (TGF) $\beta$ is involved in various fibrotic processes and IPF patients have increased levels of TGF- $\beta$ in their lungs (41) that is mainly produced by alveolar macrophages, neutrophils, AEC as well as (myo)fibroblasts (42). TGF$\beta 1$ is secreted as inactive precursor protein bound to a latency-associated protein (43) that can be released upon oxidation (44) or cleavage by metallo-proteinases (45) resulting in a biologically active form of TGF- $\beta 1$. Active TGF- $\beta$ binds to type II receptor (TGF $\beta R-I I)$ on the cell membrane, which activates type I receptor (TGFBR-I), leading to phosphorylation of Smad2 and Smad3. Phosphorylated Smad2 and Smad3 form a complex with Smad4, which then translocates to the nucleus to regulate gene transcription through binding to the promoter region of several target genes such as collagen and fibronectin (46). This eventually initiates several pro-fibrotic mediated pathways including fibroblast activation, proliferation and differentiation into myofibroblasts as well as AEC death (7). TGF- $\beta$ is also an inducer of EMT (47) and initiates the recruitment of circulating fibrocytes and progenitor cells to the lung as well as the activation of resident fibroblasts (48) thereby promoting excessive collagen deposition in the lungs of IPF patients. 
TGF- $\beta$ also initiates oxidant production from mitochondria (49) as well as NADPH oxidases (50), and contributes to a disturbed redox homeostasis in IPF.

\section{The disturbed redox-balance in IPF}

\section{Reactive oxygen species and antioxidants - the balance is important}

Reactive oxygen species (ROS), including hydrogen peroxide $\left(\mathrm{H}_{2} \mathrm{O}_{2}\right)$, superoxide anion $\left(\mathrm{O}_{2}{ }^{\circ}\right)$ and hydroxyl radical $(\mathrm{OH} \bullet)$, are derived from oxygen $\left(\mathrm{O}_{2}\right)$ and formed during the partial reduction of $\mathrm{O}_{2} . \mathrm{O}_{2}{ }^{\circ-}$ is generated by one-electron reduction of $\mathrm{O}_{2}$, which can be converted into $\mathrm{H}_{2} \mathrm{O}_{2}$ by superoxide dismutase (SOD) 1 in the cytosol and SOD3 extracellularly. $\mathrm{O}_{2}{ }^{-{ }^{-}}$ generated in the mitochondria can be converted by SOD2. $\mathrm{OH} \bullet$ is produced when $\mathrm{H}_{2} \mathrm{O}_{2}$ reacts with metal cations $\left(\mathrm{Fe}^{2+}\right.$ or $\mathrm{Cu}^{2+}$ ) via the Fenton reaction or is spontaneously formed and can cause irreversible damage to DNA and lipids. To prevent the formation of $\mathrm{OH} \bullet$, $\mathrm{H}_{2} \mathrm{O}_{2}$ is converted to $\mathrm{H}_{2} \mathrm{O}$ by various enzymatic systems, including glutathione peroxidase (GPX), peroxiredoxin (PRX) and catalase (CAT) (Figure 1.4).

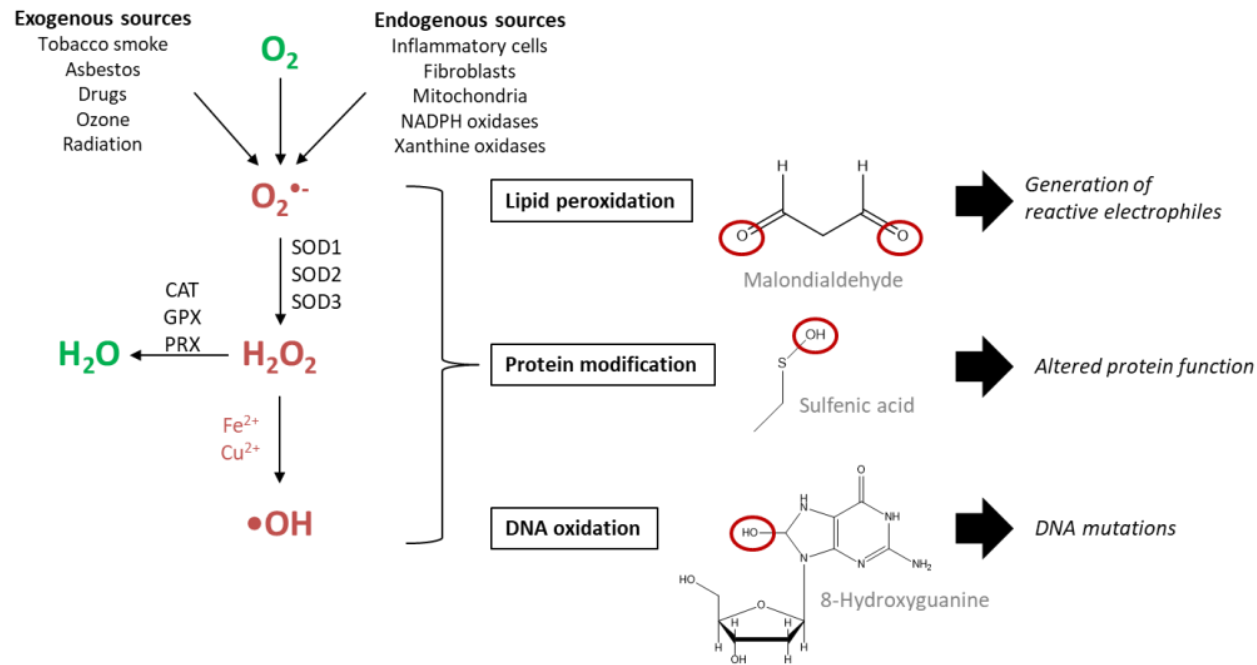

Figure 1.4: Involvement of ROS in lipid peroxidation, DNA oxidation and protein modification. Exogenous and Endogenous sources of ROS can generate superoxide $\left(\mathrm{O}_{2}{ }^{--}\right)$which can be converted into hydrogen peroxide $\left(\mathrm{H}_{2} \mathrm{O}_{2}\right)$ by superoxide dismutase (SOD). $\mathrm{H}_{2} \mathrm{O}_{2}$ can detoxified to water $\left(\mathrm{H}_{2} \mathrm{O}\right)$ by peroxiredoxins (PRX), glutathione peroxidases (GPX) and catalase (CAT). In addition, $\mathrm{H}_{2} \mathrm{O}_{2}$ can react with metal ions to generate a hydroxyl radical. The produced ROS can cause (ir)reversible damage to lipids, proteins and DNA thereby affecting cell survival and cell signaling.

Since the lungs are continuously exposed to high concentrations of $\mathrm{O}_{2}$, they are especially vulnerable to ROS-induced damage. ROS can be formed by several exogenous sources such as environmental pollutants, including tobacco smoke, asbestos fibers, drugs and radiation and are also associated with the development of interstitial lung diseases. 
Additionally, several reactive oxygen species (ROS) can be generated endogenously by various lung cell types themselves either as byproducts of cellular metabolism or by several enzyme systems such as the NADPH oxidases (NOXes).

Endogenous ROS are important in normal physiological cellular signaling and function, whereas high ROS accumulation can have deleterious biological consequences (51). Consequently, if ROS are not scavenged and neutralized, they can cause damage to biomolecules and alter protein function (Figure 1.4). For instance, ROS can contribute to genomic instability by inducing direct oxidative damage to DNA thereby potentially introducing mutations. ROS can also promote lipid peroxidation to generate mutagenic byproducts such as malondialdehyde or 4-hydroxynonenal which can adduct DNA (40). Increased ROS production can not only cause damage to macromolecules but can also lead to a redox imbalance, also called oxidative stress which is defined as an imbalance between the generation of ROS and the capacity of the cells to remove or neutralize them in favor of the first (52).

Several studies have reported that IPF patients display a higher oxidant burden compared to healthy controls. Indeed, it has been shown that pulmonary inflammatory cells in epithelial lining fluid of IPF patients generate higher levels of oxidants compared to those of healthy controls (53). Additionally, IPF patients have increased levels of $\mathrm{H}_{2} \mathrm{O}_{2}$ in exhaled breath condensate (54) as well as increased markers of lipid peroxidation such as MDA in plasma and 8-isoprostane in bronchoalveolar lavage fluid (BALF) compared to healthy non-smokers $(55,56)$. BALF from IPF patients also contains a higher amount of oxidized proteins, e.g. carbonylated proteins $(57,58)$, and epithelial cells show increased levels of DNA oxidation, illustrated by 8-hydroxy-deoxyguanosine (8-OHdG) (59).

Since ROS regulate a wide variety of physiological cell signaling, it is important they are tightly regulated by antioxidant systems to prevent oxidative stress. To combat the deleterious effects of high ROS levels, the lung has specialized antioxidant systems to protect against ROS-induced cellular damage (60). Such antioxidant defense systems include high-molecular weight antioxidants (mucins), small molecular weight antioxidants (vitamins and glutathione (GSH)), metal binding proteins (transferring, lactoferrin), thiolcontaining proteins (thioredoxin (TRX), glutaredoxin (GRX)), enzymes that degrade ROS (CAT, SOD) and phase 2 detoxifying enzymes are secreted into the airways (60). Impaired induction of these antioxidant systems can result in an overload of ROS. In IPF, there is emerging evidence that alterations in the antioxidant defense system contribute to the increased oxidant burden. Patients with IPF have a decreased level of GSH in the epithelial lining fluid (ELF) $(61,62)$ as well as in blood $(63,64)$ and $\operatorname{BALF}(55)$. It has also been shown that IPF patients have a decreased expression of SOD3 (65) and that TRX and PRXII are decreased in the lungs of patients with fibrosis compared to controls $(66,67)$. Recently, it has been shown that GRX1 mRNA expression as well as enzymatic activity are decreased in patients with IPF compared to non-IPF individuals (68). 
Many of these antioxidative enzymes are regulated through redox-sensitive transcription factors such as nuclear factor eryhroid 2 releated factor 2 (Nrf2) (69). Under normal conditions, Nrf2 is bound to its inhibitory part Kelch-like ECH-associated protein (Keap) 1 in the cytoplasm. However, in the presence of oxidative stress, the redox state of Keap1 is changed which induces the phosphorylation and subsequent dissociation of Nrf2 (70). Next, Nrf2 translocates into the nucleus where it will bind to the antioxidant response elements (ARE) that will mediate the transcription of antioxidant genes such as hemeoxygenase 1 (HO-1), glutathione-S-transferase (GST), NADP(H) quinone oxidoreductase 1 (NQO1), thioredoxin and $\gamma$ - glutamylcysteine synthetase (GCS) as compensatory mechanism for the altered redox balance (60). Animal studies support the importance of Nrf2 in IPF development, since Nrf2-deficient mice develop more fibrosis in response to bleomycin (71) and AECs isolated from Nrf2-deficient mice are more prone to oxidantinduced cell death (72).

Taken together, it can be concluded that IPF patients have decreased antioxidant levels and diminished expression of various antioxidant enzymes. These lowered antioxidative defense levels indicate that the compensation mechanisms effective are not enough to compensate for the already higher oxidative burden, making the lungs susceptible towards more ROS damage.

\section{Intracellular ROS sources}

It has been indicated that the increased oxidative stress in IPF is due to two critical players within the pathology of the disease, namely mitochondrial dysfunction, accompanied by increased mitochondrial ROS (mtROS) generation and the enhanced expression or activation of NOXes $(40,73)$.

\section{Mitochondria and mitochondrial dysfunction in IPF}

Mitochondria, the powerhouses of cells, generate ROS as byproduct in the electron transport chain (ETC), during which $\mathrm{O}_{2}$ is reduced to $\mathrm{H}_{2} \mathrm{O}$ to produce ATP, also called oxidative phosphorylation. Electrons can leak at 11 sites in the ETC mainly in complex I,II or III (74). Lung cells contain varying mitochondrial densities according to their energy needs. Mitochondria are between 0.75 and $3 \mu \mathrm{M}$ in diameter, however, cells that are metabolically more active such as ciliated epithelial cells, club cells and AECII involved in surfactant production have bigger and more mitochondria. More specifically, mitochondria are approximately three times greater in $\mathrm{AECIl}$ compared to $\mathrm{AECl}(75,76)$. In response to cellular stress or injury, mitochondria can rapidly adapt their behavior by changing mitochondrial fusion (merging) and fission (division) dynamics which will alter the ATP production demand of the cell (77). Increased fusion promotes the formation of elongated mitochondria and is a stress-resolving mechanism, occurring in response to cell injury, in which the fused mitochondria are protected from degradation to respond to the cells higher energy demand to repair the damage (78). Increased fission or reduced fusion 
on the other hand increases mitochondrial fragmentation. During differentiation from type II to type I AECs, as it occurs during wound repair, type II AECs reduce the number and size of mitochondria to adapt to cellular stress, which results in lower energy demanding type I AECs (75). If this adaption fails to be effective, lung cells become more vulnerable to injury and oxidative stress thereby promoting pulmonary fibrosis. Intriguingly, various lines of evidence have suggested that IPF patients have an increased number of dysfunctional mitochondria characterized by a decreased efficiency of the ETC, increased mtROS production, decreased mitochondrial biogenesis and impaired breakdown of dysfunctional mitochondria (mitophagy) (79).

Although enhanced generation of mtROS is considered a feature of mitochondrial dysfunction, there is growing evidence supporting a role for mtROS as signaling molecules. Recent studies indicate oxidant cross-talk between mitochondria and NOX4, possibly leading to altered activity of both players through ROS-induced ROS release (80). Such interactions between NOX and mitochondria are reciprocal, i.e. activation of NOX can increase mtROS production but mitochondria (perhaps via mtROS) can also contribute to activation of NOX, indicating a complex feedback mechanism.

The NOX family and their involvement in IPF

Recent evidence suggests a role for ROS-producing NOX enzymes in the oxidative stress observed in IPF pathogenesis (81). The NOX family consists of seven NOX homologs, NOX1-5 and Dual Oxidase (DUOX) 1 and DUOX2. NOX proteins differ from each other regarding their activation and subcellular localization, but they also produce distinct ROS and regulate different processes $(82,83)$. Activation of NOX enzymes generates $\mathrm{O}_{2}{ }^{\circ-}$ or $\mathrm{H}_{2} \mathrm{O}_{2}$ as primary products that can react with redox-sensitive proteins, thereby regulating various cellular processes including cell proliferation, differentiation, inflammatory signaling and host defense.

NOX enzymes that have been reported to contribute to pulmonary fibrosis are NOX1 (84, 85), NOX2 $(86,87)$ and NOX4 $(50,88-90)$. NOX4 is the only isoform that is highly upregulated in IPF lungs, mainly in fibroblasts and epithelial cells, but also in endothelial and smooth muscle cells, NOX4-derived ROS, mainly $\mathrm{H}_{2} \mathrm{O}_{2}$ (69), are required to induce AEC death, differentiation of fibroblasts into myofibroblasts and activation of myofibroblasts, ultimately leading to collagen deposition in the lungs. NOX4 needs association with $\mathrm{p} 22^{\text {phox }}$ but does not require any other cofactors for its activation and is thought to be constitutively active (91) (Figure 1.5). 


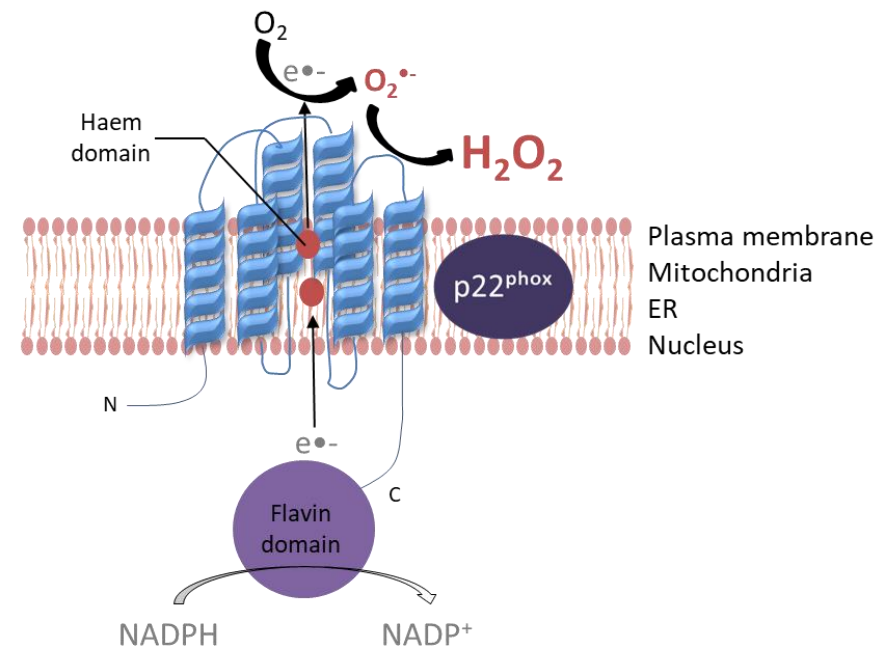

Figure 1.5: Structural overview of NOX4. NOX4 has six trans-membrane spanning alpha helices with cytosolic N and C-termini. The C-terminal flavoprotein domain contains an NADPH-binding region and a flavin adenine dinucleotide binding region whereas the $\mathrm{N}$-terminal hydrophobic domain contains two heme-binding sites (92). Through the membrane-associated flavocytochrome $\mathrm{p} 22^{\mathrm{phox}}$, transmembrane electron transfer takes place from $\mathrm{NADPH}$ to reduce $\mathrm{O}_{2}$ to superoxide $\left(\mathrm{O}^{2 \bullet-}\right)$ and hydrogen peroxide $\left(\mathrm{H}_{2} \mathrm{O}_{2}\right)$.

Induction of NOX4 is primarily mediated by the profibrotic cytokine TGF- $\beta 1$ which is also upregulated in IPF. Interestingly, NOX4-generated ROS promote AEC death on the one hand but induce senescence of myofibroblasts on the other hand leading to an apoptosis resistant myofibroblast phenotype. The molecular mechanisms by which NOX4 promotes these pro-fibrotic outcomes are not clear yet, but various studies indicate that NOX4dependent production of $\mathrm{H}_{2} \mathrm{O}_{2}$ can regulate cell signaling pathways that control proliferation, migration, differentiation, and or survival, by inducing specific redoxmediated post-translational modifications to redox-sensitive proteins such as protein kinases (93).

\section{Redox regulation in IPF}

\section{Redox signaling - an overview}

During the past two decades, it has become clear that ROS, when present in nM range, also act as messenger in cell signaling. The reversible oxidation of protein cysteine residues, also called redox switches, is widely recognized as an important mechanism in redox signaling (94). Both superoxide and hydrogen peroxide have been implicated as potential messengers, however, $\mathrm{H}_{2} \mathrm{O}_{2}$ it is more likely involved because of its stability $(95,96)$.

Cysteine residues exist as thiolate anions (Cys- $\left.\mathrm{S}^{-}\right)$at physiological $\mathrm{pH}$ and are more susceptible to oxidation than the protonated cysteine thiols (Cys-SH). During redox 
signaling, $\mathrm{H}_{2} \mathrm{O}_{2}$ can oxidize thiol groups in cysteine residues within redox-sensitive proteins, thereby affecting protein function and cellular signaling (95). $\mathrm{H}_{2} \mathrm{O}_{2}$ oxidation at the thiolate anion to form sulfenic acid $(-\mathrm{SOH})$ affects cellular signaling by altering protein confirmation as well as protein activity if the oxidized cysteine is located in its catalytic domain. When two or more cysteine residues within the same protein are oxidized, they may form an intramolecular disulfide bridge, thereby altering the confirmation and thus activity of the protein. Sulfenic acid can also react with GSH, forming a gluthathione protein disulfide, which is called glutathionylation (97). The sulfenic form can be reduced to thiolate anions by thioredoxin ( $T r x)$ and glutaredoxin (Grx), thereby changing the confirmation of the protein back to its original state (98). Sulfenic acid can further be oxidized, in the presence of high $\mathrm{H}_{2} \mathrm{O}_{2}$ concentration, to form sulfinic $\left(-\mathrm{SO}_{2} \mathrm{H}\right)$ and sulfonic acid $\left(-\mathrm{SO}_{3} \mathrm{H}\right)$ in an irreversible reaction. Hence, first-degree oxidation of cysteine residues within proteins serves as a reversible signal transduction mechanism whereas the formation of sulfinic and sulfonic acid is irreversible and results in permanent protein damage (Figure 1.6).

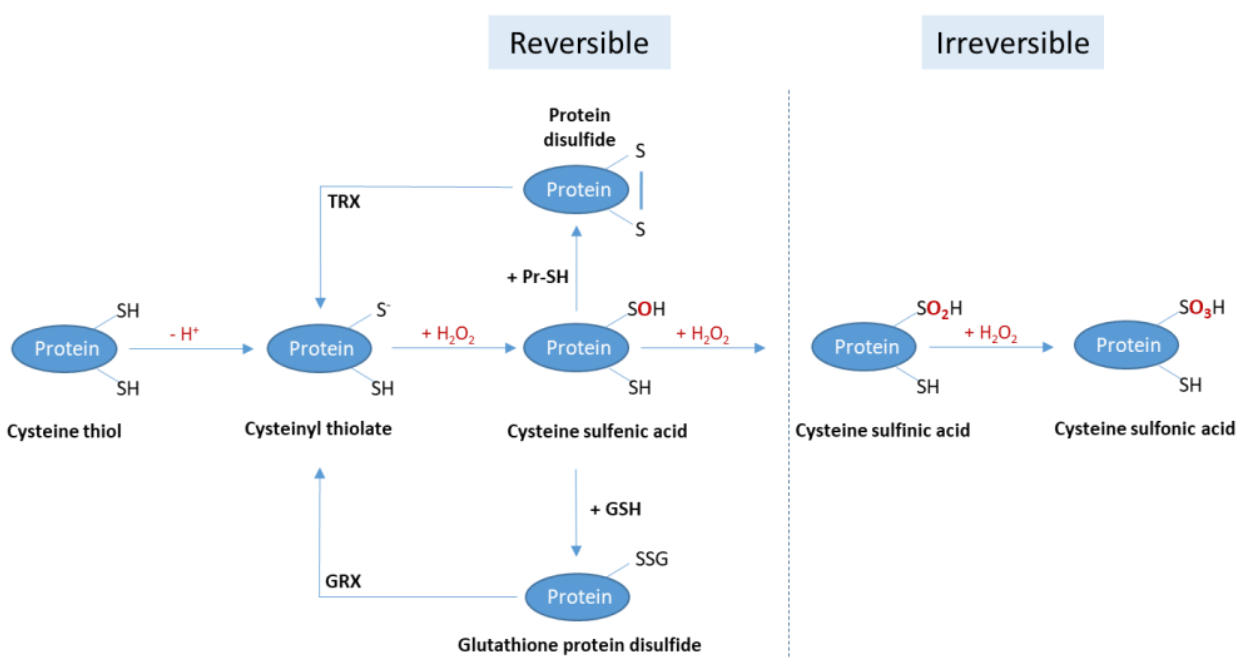

Figure 1.6: Oxidative modification of thiol groups within cysteine residues. ROS can oxidize cysteine thiols (-SH) to sulfenic acid (-SOH) which can react with reduced thiols ( $\mathrm{Pr}-\mathrm{SH}$ ) or GSH to form protein disulfides. This process is reversible, and the protein can be reduced back by thioredoxin (TRX) or glutaredoxin (GRX) respectively. Moreover, in the presence of excess ROS, sulfenic acid can be further oxidized into sulfinic $\left(-\mathrm{SO}_{2} \mathrm{H}\right)$ or sulfonic acid $\left(-\mathrm{SO}_{3} \mathrm{H}\right)$.

Taken together, oxidant signaling is important for protein activity and increasing evidence suggests that IPF is associated with dysregulated redox signaling rather than solely oxidative stress. One example of proteins regulated by redox signaling is the family of SRC kinases that have recently also been implicated in the development of pulmonary fibrosis. 


\section{Redox sensitive proteins: SRC family kinase}

SRC family kinases (SFK) are a family of non-receptor tyrosine kinases with nine members, namely: SRC, YES, FYN, FGR, LCK, HCK, BLK, FRK and LYN, of which SRC, FYN and Yes are ubiquitously expressed $(99,100)$. They are involved in various signaling pathways important for cellular homeostasis, including cell differentiation and proliferation (101, 102) and are dysregulated in the pathogenesis of IPF (103). It has been shown that SFKs are activated in response to TGF- $\beta$ and recent studies have indicated, that SFKs are important players in the development of lung fibrosis (104). It has also been reported that SFKs regulate EMT, which is important for wound healing, a process that is often disturbed in IPF $(105,106)$. Intriguingly, inhibition of SRC kinases by AZD0530 in an experimental model of pulmonary fibrosis reduced the fibrotic lesions as well as collagen deposition, two pathological hallmarks of IPF, in the lungs of mice after bleomycin instillation (104, 107).

All members of the SFK share a similar domain structure consisting of a SH3, SH2 and kinase domain that harbors the activation loop and is important for the full activity of the kinase (108). The activity of SFKs is regulated through its phosphorylation status. In an inactive state, SFKs are phosphorylated at Tyr527 (in SRC) in the C-terminal region and dephosphorylated at Tyr416 (in SRC) in the kinase domain, resulting in a close interaction between the $\mathrm{SH} 2$ domain and tail region. Upon stimulation, Tyr527 becomes dephosphorylated causing a conformational change and autophosphorylation of Tyr416, a combination that ultimately leads to full activity of the kinase (109) (Figure 1.7).
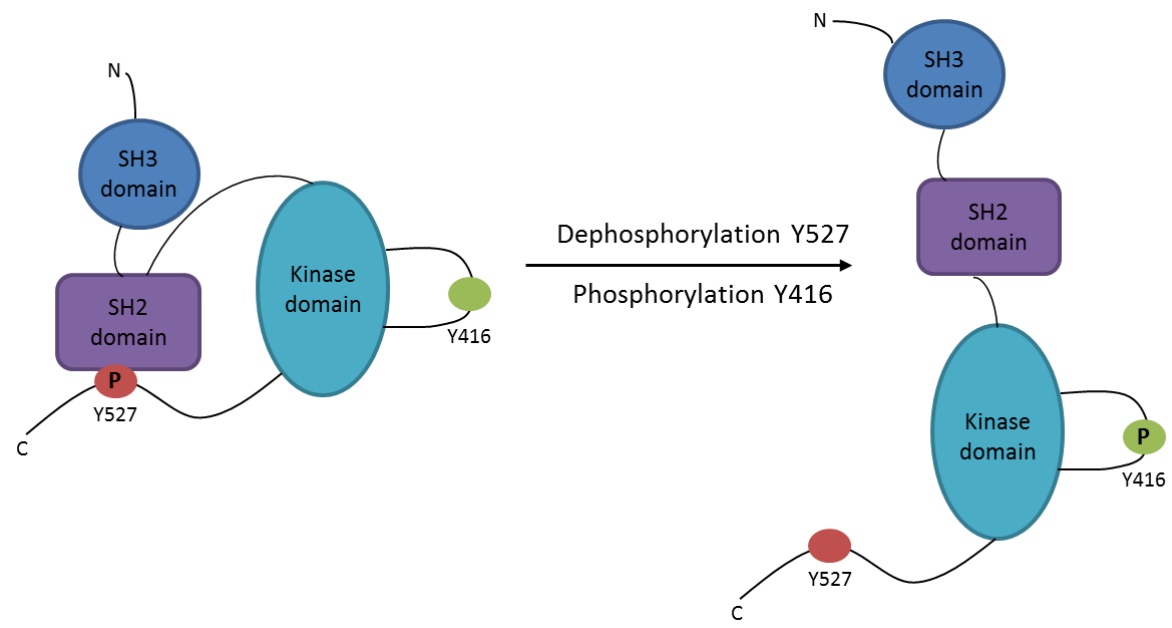

Figure 1.7: Canonical activation mechanism of SRC kinases. The activity of SRC kinases is regulated through control of the phosphorylation state. In an active state tyrosine 527 is dephosphorylated leading to "unclamping" of the protein and autophosphorylation of tyrosine 416 in the activation loop. Amino acid numbering of SRC is based on the avian SRC sequence. 
Additionally, studies have shown that SFKs can also be activated through a redoxdependent, non-canonical pathway that is not dependent on the dephosphorylation of Tyr530 $(100,110)$. Here, it is speculated that one or more conserved cysteine residues within the $\mathrm{SH} 2$ or kinase domain become oxidized by $\mathrm{O}_{2}{ }^{-}$or $\mathrm{H}_{2} \mathrm{O}_{2}$, which are also produced in response to TGF- $\beta 1$. Oxidation of these cysteine residues forms reactive sulfenic acid $(-\mathrm{SOH})$ that can form disulfide bonds with nearby cysteines (-S-S-). These oxidative modifications can facilitate autophosphorylation of Tyr416, leading to full activation of SFKs thereby affecting the function of the protein (109) (Figure 1.8). As stated earlier, intracellular ROS sources include NOX enzymes as well as the mitochondria.

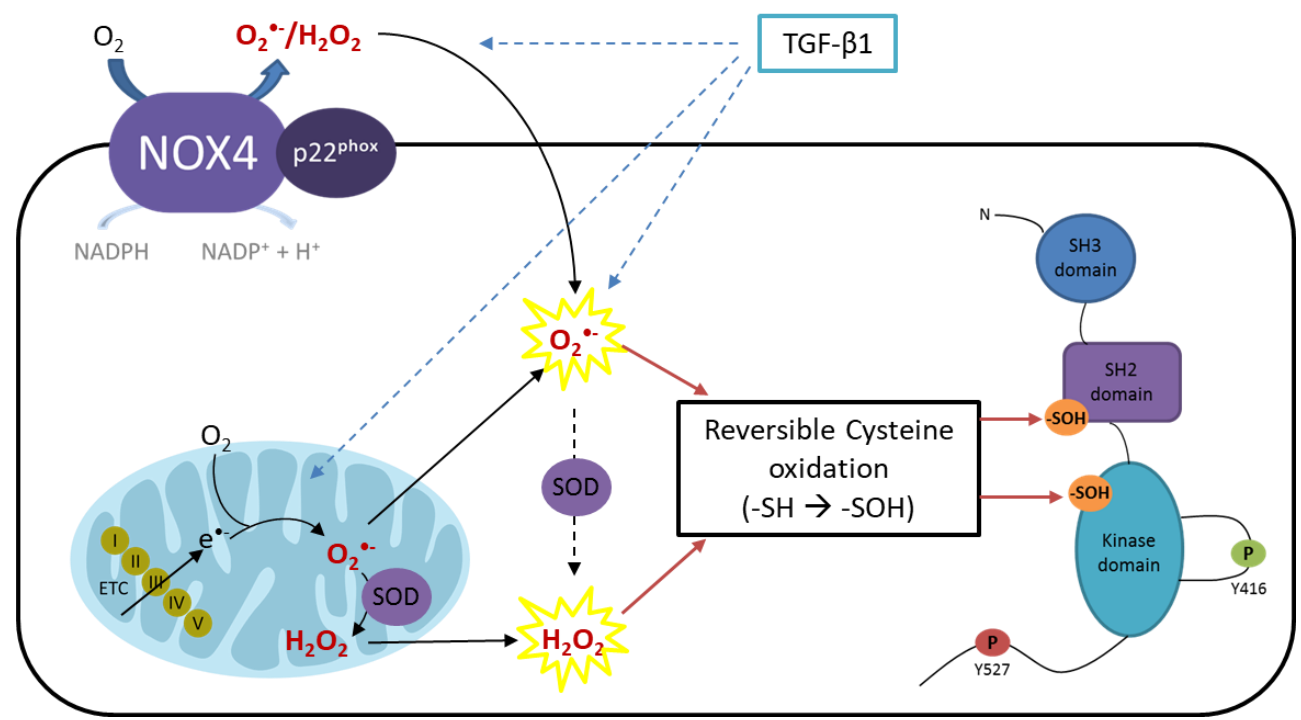

Figure 1.8: Non-canonical activation mechanism of SRC kinases. SRC kinases can also get activated independent of Y527 phosphorylation through oxidation of cysteine residues. TGF- $\beta 1$ induces ROS production by activation of NOX enzymes as well as upregulation of mitochondrial ROS leading to the oxidation of thiol groups on cysteine residues forming sulfenic acid thereby affecting the activity of the protein.

Interestingly, the SFK members SRC and FYN have been shown to translocate to mitochondria where they can regulate their metabolism through phosphorylation of the mitochondrial subunits I,II and IV and pyruvate dehydrogenase (111-113). Recent evidence also indicates that FYN-dependent phosphorylation is involved in mitochondrial protein synthesis and regulation of oxidative phosphorylation (114). Moreover, FYN is also known to interact with NOX4 in the mitochondria of cardiomyocytes where it acts as a ROS sensor and regulates cell death (115). However, the specific role of members of the SFK in the development of IPF is still not clear. 


\section{Treatment strategies in IPF}

\section{Anti-fibrotic therapies}

Until now, there are only two U.S. Food and Drug Administration (FDA)-approved drugs available for IPF treatment, pirfenidone (Esbriet ${ }^{\circledR}$; Roche/Genentech USA) and nintedanib (Ofev ; Boehringer Ingelheim). Next to pirfenidone and nintedanib to slow down disease progression, several symptomatic therapies are also implemented in IPF treatment such as oxygen therapy (to maintain oxygen saturation above 90\%), anti-reflux therapy and treating obstructive sleep apnea as this is a risk factor for IPF. Despite all these additional options, there are no therapies that can currently prolong life other than lung transplantation (116).

Pirfenidone (Esbriet ${ }^{\oplus}$; Roche/Genentech USA) has anti-oxidative, anti-inflammatory and anti-fibrotic effects although the exact working mechanisms underlying these effects are not clear yet. Nintedanib (Ofev ; Boehringer Ingelheim) is an intracellular tyrosine kinase inhibitor, which mainly inhibits the platelet-derived growth factor receptor (PDGFR), the fibroblast growth factor receptor (FGFR) and vascular endothelial growth factor receptor (VEGFR) as well as non-receptor tyrosine kinases of the SRC family (117). Both of them mainly target the activation of fibroblasts to reduce collagen deposition in the lungs. Although both drugs can slow down the progression of IPF to some extent, they cannot reverse the scarring of the lungs or stop the disease deterioration, implying that there is no effective cure until now. Moreover, it is still not understood why these drugs do not work in all patients and which drug could exert the most health beneficial effects in which patients. Since the effects markedly differ between patients, treatment strategies should focus on personalized treatment (118).

\section{Proposed redox-modulatory therapies}

\section{$\mathrm{N}$-acetyl cysteine}

Following the important role of oxidant regulation in IPF, several therapeutic strategies focused at directly inhibiting oxidant generation as well as stimulating antioxidant production to regulate the disturbed redox-balance. Various treatments such as the GSH precursor $\mathrm{N}$-acetyl-cysteine (NAC) have demonstrated promising results in various in vitro studies (119) as well as in experimental models of pulmonary fibrosis $(120,121)$. However it failed to be fully effective in the clinic (122-124) because of variable effects in patients with IPF. Interestingly, NAC has also been shown to inhibit SFK activity (125).

\section{Dietary antioxidant supplements}

Antioxidant food supplements including quercetin and resveratrol, which are also Nrf2 activators, reduce oxidative stress in profibrotic responses in vitro $(63,126-128)$ as well as in animal models (129-132) of pulmonary fibrosis highlighting the important role of a the dysregulated redox homeostasis in the development of IPF. 
Quercetin is one of the most abundant flavonoids in nature, mainly present in onions and apples. The average intake of quercetin is approximately $16 \mathrm{mg} /$ day in the Netherlands (133). Flavonoids are polyphenolic compounds and known because of their antioxidant properties indicated by a trolox equivalent antioxidant capacity of $4.7 \mathrm{nM}$ (134). This antioxidant capacity is mainly due to the presence of hydroxyl groups at 3' and 4' position of the $B$ ring as well as hydroxyl groups in the $3^{\prime}$ position in the $C$ ring and $5^{\prime}$ and $7^{\prime}$ position of the A ring. Quercetin is a potent scavenger of especially superoxide (135) due to the presence of these hydroxyl groups. Upon the oxidation of one or more of the hydroxyl groups, quercetin quinones are formed. Quercetin has four tautomeric forms of its oxidation product; an ortho-quinone and three different quinone-methides (Figure 1.9).
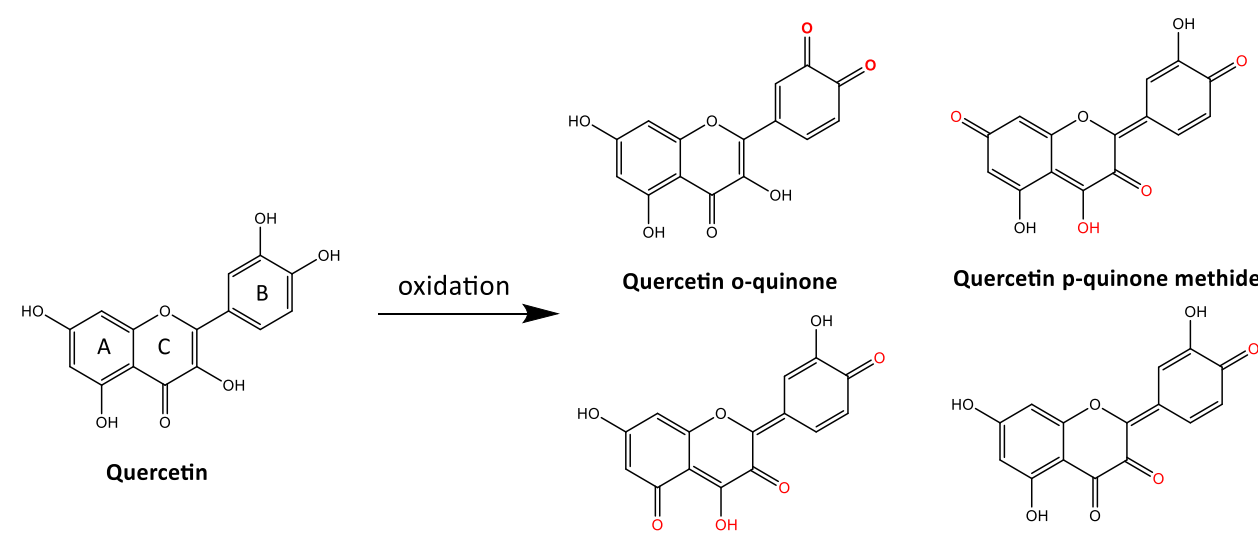

Quercetin p-quinone methide I

Quercetin p-quinone methide II

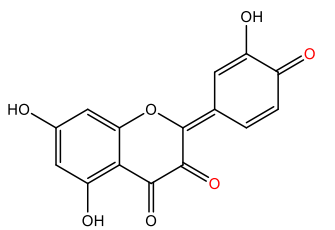

Quercetin p-quinone methide III

Figure 1.9: Chemical structure of quercetin and tautomeric forms of the quinone structure of quercetin.

Quercetin quinones can have toxic effects due to their reactivity towards thiol groups. Indeed, quercetin can react with GSH and protein thiols which may lead to changes in protein confirmation and thus have consequences for their activity (136).

Next to its antioxidant capacities, quercetin has also been shown to possess antiinflammatory (137), anti-proliferative (138), immune-suppressive (139), anti-fibrotic (140) as well as Nrf2-inducing properties (141). Very recently, it has also been shown that quercetin can increase apoptosis in senescent fibroblasts from IPF patients (142). However, several studies have speculated that quercetin is not only exerting its effects because of its antioxidant capacity, but also as the flavonoid interferes with cell signaling cascades that may be of even more importance (143). More specifically, it has been shown that quercetin can inhibit PI3K as well as SRC kinase (144) through binding to their ATP binding sites. However, their precise mechanism of action is still unclear.

A better understanding of the mechanisms mediating the oxidant-antioxidant imbalance in IPF is the first critical step towards the development of more effective redox- 
modulatory therapeutic strategies. Rather than the systemically scavenging of ROS that has been tried so far without much success, it may be more effective to specifically target the dysregulated redox homeostasis. 


\section{Aims and outline of the thesis}

An imbalance between oxidants and antioxidants has been implicated in many diseases, including pulmonary fibrosis. However, it is still not completely clear how redox regulation contributes to the progression of this disease.

To obtain better insights in the role of oxidant dysregulation in the pathology of idiopathic pulmonary fibrosis, several studies were performed and described in this thesis. The overall aim of this thesis was to explore the importance of oxidant regulation in pulmonary fibrosis. The main focus was to study the role of reactive oxygen species (ROS) in general and oxidants produced by the NADPH oxidase NOX4 specifically in various in vitro and in vivo models of pulmonary fibrosis. Furthermore, various redox-modulatory approaches were explored to mitigate specific features of IPF.

Following this introduction and overall aim of the thesis, an introduction on redoxsignaling in IPF, accompanied by an overview of current and possible future treatment strategies, is given in Chapter 2. In this review, the various sources of ROS and their proposed roles in the development of IPF are discussed as well as the oxidant crosstalk between NOX and mitochondria that may give rise to potential future treatment strategies for IPF.

In Chapter 3 we examine the potentially protective effects of the dietary antioxidant quercetin on markers of oxidative stress and inflammation in human bronchial epithelial cells and in blood of IPF patients. Our findings implicate that IPF patients may benefit from the use of quercetin to restore the disturbed redox balance and reduce the occurring inflammation. Chapter $\mathbf{4}$ describes the effects of quercetin in a bleomycin-induced murine model of pulmonary fibrosis in Nrf2 wildtype and Nrf2 knockout mice. Our results show that quercetin induces pulmonary gene expression of $\mathrm{Nrf2}$ and Nrf2-responsive genes compared to non-quercetin fed mice. Upon bleomycin treatment, quercetin-fed mice display reduced expression of collagen and fibronectin and as well as reduced pulmonary inflammatory lesions, which was dependent on the presence of Nrf2. Furthermore, quercetin reduces pulmonary gene expression of TNF $\alpha$ and KC, but not their plasma levels, and diminishes malondialdehyde-dG DNA adducts.

NADPH oxidase NOX4 is an important source of ROS in IPF we investigate the role of NOX4 in the activation of SRC family kinases in Chapter 5. The SRC family kinase member FYN is activated through NOX4-dependent oxidant signaling and inhibition of FYN reduces mitochondrial ROS production, leading to reduction of profibrotic markers in human bronchial epithelial cells. Chapter 6 describes the role of SFK in human fibroblasts upon stimulation with the profibrotic cytokine TGF- $\beta 1$. Silencing of the SFK member YES reduces activation of SFK and TGF- $\beta 1$ induced extracellular matrix genes as well as gene expression of NOX4. 
Bronchial epithelial cell from IPF patients demonstrate an increased activation of SFK as well as decreased expression of genes associated with mitochondrial health, which is depicted in Chapter 7. Inhibition of SFK by the pharmacological inhibitor AZD reduced the increased activation of SFK and increased expression of genes related to mitochondrial biogenesis and mitophagy. In Chapter $\mathbf{8}$ we compare pirfenidone and nintedanib to the SFK inhibitor AZD in differentiated human bronchial epithelial cells from IPF patients. Our data suggest that responsiveness to treatment varies substantially between individual IPF patients, thereby indicating that IPF patients might benefit from personalized treatment. Finally, the results as well as the implications and limitations of our findings are discussed in Chapter 9. 


\section{References}

1. Silverthorn DU. Human Physiology: an integrated approach. Mechanics of Breathing 5: Pearson; 2010. p. 569-74.

2. Chuquimia OD, Petursdottir DH, Periolo N, Fernandez C. Alveolar epithelial cells are critical in protection of the respiratory tract by secretion of factors able to modulate the activity of pulmonary macrophages and directly control bacterial growth. Infection and immunity. 2013;81(1):381-9.

3. McCauley HA, Guasch G. Three cheers for the goblet cell: maintaining homeostasis in mucosal epithelia. Trends Mol Med. 2015;21(8):492-503.

4. Rokicki W, Rokicki M, Wojtacha J, Dzeljijli A. The role and importance of club cells (Clara cells) in the pathogenesis of some respiratory diseases. Kardiochir Torakochirurgia Pol. 2016;13(1):26-30.

5. Whitsett JA, Alenghat T. Respiratory epithelial cells orchestrate pulmonary innate immunity. Nat Immunol. 2015;16(1):27-35.

6. Effros RM. Anatomy, development, and physiology of the lungs. GI Motility online 2006.

7. Camelo A, Dunmore R, Sleeman MA, Clarke DL. The epithelium in idiopathic pulmonary fibrosis: breaking the barrier. Front Pharmacol. 2014;4:173.

8. Konigshoff M. Lung cancer in pulmonary fibrosis: tales of epithelial cell plasticity. Respiration; international review of thoracic diseases. 2011;81(5):353-8.

9. Ghosh MC, Gorantla V, Makena PS, Luellen C, Sinclair SE, Schwingshackl A, et al. Insulinlike growth factor-I stimulates differentiation of ATII cells to ATI-like cells through activation of Wnt5a. American journal of physiology Lung cellular and molecular physiology. 2013;305(3):L222-8. 10. American Thoracic Society. Idiopathic pulmonary fibrosis: diagnosis and treatment. International consensus statement. American Thoracic Society (ATS), and the European Respiratory Society (ERS). Am J Respir Crit Care Med. 2000;161(2 Pt 1):646-64.

11. King TE, Jr., Pardo A, Selman M. Idiopathic pulmonary fibrosis. Lancet. 2011;378(9807):1949-61.

12. Raghu G, Weycker D, Edelsberg J, Bradford WZ, Oster G. Incidence and prevalence of idiopathic pulmonary fibrosis. Am J Respir Crit Care Med. 2006;174(7):810-6.

13. Nalysnyk L, Cid-Ruzafa J, Rotella P, Esser D. Incidence and prevalence of idiopathic pulmonary fibrosis: review of the literature. Eur Respir Rev. 2012;21(126):355-61.

14. Navaratnam V, Fleming KM, West J, Smith CJ, Jenkins RG, Fogarty A, et al. The rising incidence of idiopathic pulmonary fibrosis in the U.K. Thorax. 2011;66(6):462-7.

15. Collard HR. The age of idiopathic pulmonary fibrosis. Am J Respir Crit Care Med. 2010;181(8):771-2.

16. Lopez-Otin C, Blasco MA, Partridge L, Serrano M, Kroemer G. The hallmarks of aging. Cell. 2013;153(6):1194-217.

17. Evans CM, Fingerlin TE, Schwarz MI, Lynch D, Kurche J, Warg L, et al. Idiopathic Pulmonary Fibrosis: A Genetic Disease That Involves Mucociliary Dysfunction of the Peripheral Airways. Physiol Rev. 2016;96(4):1567-91.

18. Seibold MA, Wise AL, Speer MC, Steele MP, Brown KK, Loyd JE, et al. A common MUC5B promoter polymorphism and pulmonary fibrosis. N Engl J Med. 2011;364(16):1503-12.

19. Mora AL, Rojas M, Pardo A, Selman M. Emerging therapies for idiopathic pulmonary fibrosis, a progressive age-related disease. Nat Rev Drug Discov. 2017;16(11):810.

20. Kosmider B, Messier EM, Chu HW, Mason RJ. Human alveolar epithelial cell injury induced by cigarette smoke. PLoS One. 2011;6(12):e26059.

21. Liu G, Beri R, Mueller A, Kamp DW. Molecular mechanisms of asbestos-induced lung epithelial cell apoptosis. Chem Biol Interact. 2010;188(2):309-18. 
22. Kamp DW, Panduri V, Weitzman SA, Chandel N. Asbestos-induced alveolar epithelial cell apoptosis: role of mitochondrial dysfunction caused by iron-derived free radicals. Mol Cell Biochem. 2002;234-235(1-2):153-60.

23. Hays LE, Zodrow DM, Yates JE, Deffebach ME, Jacoby DB, Olson SB, et al. Cigarette smoke induces genetic instability in airway epithelial cells by suppressing FANCD2 expression. Br J Cancer. 2008;98(10):1653-61.

24. Selman M, King TE, Pardo A, American Thoracic S, European Respiratory S, American College of Chest P. Idiopathic pulmonary fibrosis: prevailing and evolving hypotheses about its pathogenesis and implications for therapy. Ann Intern Med. 2001;134(2):136-51.

25. Kurundkar A, Thannickal VJ. Redox mechanisms in age-related lung fibrosis. Redox Biol. 2016;9:67-76.

26. Jolly MK, Ward C, Eapen MS, Myers S, Hallgren O, Levine H, et al. Epithelial-mesenchymal transition, a spectrum of states: Role in lung development, homeostasis, and disease. Dev Dyn. 2018;247(3):346-58.

27. Minagawa S, Araya J, Numata T, Nojiri S, Hara H, Yumino Y, et al. Accelerated epithelial cell senescence in IPF and the inhibitory role of SIRT6 in TGF-beta-induced senescence of human bronchial epithelial cells. Am J Physiol Lung Cell Mol Physiol. 2011;300(3):L391-401.

28. Pardo A, Selman M. Idiopathic pulmonary fibrosis: new insights in its pathogenesis. Int J Biochem Cell Biol. 2002;34(12):1534-8.

29. Nicholson AG, Fulford LG, Colby TV, du Bois RM, Hansell DM, Wells AU. The relationship between individual histologic features and disease progression in idiopathic pulmonary fibrosis. Am J Respir Crit Care Med. 2002;166(2):173-7.

30. Waghray M, Cui Z, Horowitz JC, Subramanian IM, Martinez FJ, Toews GB, et al. Hydrogen peroxide is a diffusible paracrine signal for the induction of epithelial cell death by activated myofibroblasts. FASEB J. 2005;19(7):854-6.

31. Alvarez D, Cardenes N, Sellares J, Bueno M, Corey C, Hanumanthu VS, et al. IPF lung fibroblasts have a senescent phenotype. Am J Physiol Lung Cell Mol Physiol. 2017;313(6):L1164-L73. 32. Nuovo GJ, Hagood JS, Magro CM, Chin N, Kapil R, Davis L, et al. The distribution of immunomodulatory cells in the lungs of patients with idiopathic pulmonary fibrosis. Mod Pathol. 2012;25(3):416-33.

33. Idiopathic Pulmonary Fibrosis Clinical Research N, Raghu G, Anstrom KJ, King TE, Jr., Lasky JA, Martinez FJ. Prednisone, azathioprine, and N-acetylcysteine for pulmonary fibrosis. N Engl J Med. 2012;366(21):1968-77.

34. Jones HA, Schofield JB, Krausz T, Boobis AR, Haslett C. Pulmonary fibrosis correlates with duration of tissue neutrophil activation. Am J Respir Crit Care Med. 1998;158(2):620-8.

35. Kinder BW, Brown KK, Schwarz MI, Ix JH, Kervitsky A, King TE, Jr. Baseline BAL neutrophilia predicts early mortality in idiopathic pulmonary fibrosis. Chest. 2008;133(1):226-32.

36. Balestro E, Calabrese F, Turato G, Lunardi F, Bazzan E, Marulli G, et al. Immune Inflammation and Disease Progression in Idiopathic Pulmonary Fibrosis. PLoS One. 2016;11(5):e0154516.

37. Papiris SA, Tomos IP, Karakatsani A, Spathis A, Korbila I, Analitis A, et al. High levels of IL-6 and IL-8 characterize early-on idiopathic pulmonary fibrosis acute exacerbations. Cytokine. 2018;102:168-72.

38. Rohani MG, McMahan RS, Razumova MV, Hertz AL, Cieslewicz M, Pun SH, et al. MMP-10 Regulates Collagenolytic Activity of Alternatively Activated Resident Macrophages. J Invest Dermatol. 2015;135(10):2377-84.

39. Madsen DH, Leonard D, Masedunskas A, Moyer A, Jurgensen HJ, Peters DE, et al. M2-like macrophages are responsible for collagen degradation through a mannose receptor-mediated pathway. J Cell Biol. 2013;202(6):951-66. 
40. Bargagli E, Olivieri C, Bennett D, Prasse A, Muller-Quernheim J, Rottoli P. Oxidative stress in the pathogenesis of diffuse lung diseases: a review. Respir Med. 2009;103(9):1245-56.

41. Bergeron A, Soler P, Kambouchner M, Loiseau P, Milleron B, Valeyre D, et al. Cytokine profiles in idiopathic pulmonary fibrosis suggest an important role for TGF-beta and IL-10. Eur Respir J. 2003;22(1):69-76.

42. Fernandez IE, Eickelberg $\mathrm{O}$. The impact of TGF-beta on lung fibrosis: from targeting to biomarkers. Proc Am Thorac Soc. 2012;9(3):111-6.

43. Munger JS, Harpel JG, Gleizes PE, Mazzieri R, Nunes I, Rifkin DB. Latent transforming growth factor-beta: structural features and mechanisms of activation. Kidney Int. 1997;51(5):1376-

82.

44. Barcellos-Hoff MH, Dix TA. Redox-mediated activation of latent transforming growth factor-beta 1. Mol Endocrinol. 1996;10(9):1077-83.

45. Kobayashi T, Kim H, Liu X, Sugiura H, Kohyama T, Fang Q, et al. Matrix metalloproteinase-9 activates TGF-beta and stimulates fibroblast contraction of collagen gels. Am J Physiol Lung Cell Mol Physiol. 2014;306(11):L1006-15.

46. Derynck R, Zhang YE. Smad-dependent and Smad-independent pathways in TGF-beta family signalling. Nature. 2003;425(6958):577-84.

47. Willis BC, Liebler JM, Luby-Phelps K, Nicholson AG, Crandall ED, du Bois RM, et al. Induction of epithelial-mesenchymal transition in alveolar epithelial cells by transforming growth factor-beta1: potential role in idiopathic pulmonary fibrosis. Am J Pathol. 2005;166(5):1321-32. 48. Hong KM, Belperio JA, Keane MP, Burdick MD, Strieter RM. Differentiation of human circulating fibrocytes as mediated by transforming growth factor-beta and peroxisome proliferatoractivated receptor gamma. J Biol Chem. 2007;282(31):22910-20.

49. Jain M, Rivera S, Monclus EA, Synenki L, Zirk A, Eisenbart J, et al. Mitochondrial reactive oxygen species regulate transforming growth factor-beta signaling. J Biol Chem. 2013;288(2):770-7.

50. Amara N, Goven D, Prost F, Muloway R, Crestani B, Boczkowski J. NOX4/NADPH oxidase expression is increased in pulmonary fibroblasts from patients with idiopathic pulmonary fibrosis and mediates TGFbeta1-induced fibroblast differentiation into myofibroblasts. Thorax. 2010;65(8):733-8.

51. Emerit J, Michelson AM. [Free radicals in medicine and biology]. Sem Hop. 1982;58(45):2670-5.

52. Betteridge DJ. What is oxidative stress? Metabolism. 2000;49(2 Suppl 1):3-8.

53. Cantin AM, North SL, Fells GA, Hubbard RC, Crystal RG. Oxidant-mediated epithelial cell injury in idiopathic pulmonary fibrosis. The Journal of clinical investigation. 1987;79(6):1665-73.

54. Psathakis K, Mermigkis D, Papatheodorou G, Loukides S, Panagou P, Polychronopoulos V, et al. Exhaled markers of oxidative stress in idiopathic pulmonary fibrosis. Eur J Clin Invest. 2006;36(5):362-7.

55. Rahman I, Skwarska E, Henry M, Davis M, O'Connor CM, FitzGerald MX, et al. Systemic and pulmonary oxidative stress in idiopathic pulmonary fibrosis. Free Radic Biol Med. 1999;27(1-2):60-8.

56. Montuschi P, Ciabattoni G, Paredi P, Pantelidis P, du Bois RM, Kharitonov SA, et al. 8Isoprostane as a biomarker of oxidative stress in interstitial lung diseases. American journal of respiratory and critical care medicine. 1998;158(5 Pt 1):1524-7.

57. Lenz AG, Costabel U, Maier KL. Oxidized BAL fluid proteins in patients with interstitial lung diseases. The European respiratory journal. 1996;9(2):307-12.

58. Rottoli P, Magi B, Cianti R, Bargagli E, Vagaggini C, Nikiforakis N, et al. Carbonylated proteins in bronchoalveolar lavage of patients with sarcoidosis, pulmonary fibrosis associated with systemic sclerosis and idiopathic pulmonary fibrosis. Proteomics. 2005;5(10):2612-8.

59. Kuwano K, Nakashima N, Inoshima I, Hagimoto N, Fujita M, Yoshimi M, et al. Oxidative stress in lung epithelial cells from patients with idiopathic interstitial pneumonias. The European respiratory journal. 2003;21(2):232-40. 
60. Cho HY, Reddy SP, Kleeberger SR. Nrf2 defends the lung from oxidative stress.

Antioxidants \& redox signaling. 2006;8(1-2):76-87.

61. Cantin AM, Hubbard RC, Crystal RG. Glutathione deficiency in the epithelial lining fluid of the lower respiratory tract in idiopathic pulmonary fibrosis. Am Rev Respir Dis. 1989;139(2):370-2.

62. Meyer A, Buhl R, Magnussen $\mathrm{H}$. The effect of oral $\mathrm{N}$-acetylcysteine on lung glutathione levels in idiopathic pulmonary fibrosis. The European respiratory journal. 1994;7(3):431-6.

63. Veith C, Drent M, Bast A, van Schooten FJ, Boots AW. The disturbed redox-balance in pulmonary fibrosis is modulated by the plant flavonoid quercetin. Toxicol Appl Pharmacol. 2017;336:40-8.

64. Muramatsu Y, Sugino K, Ishida F, Tatebe J, Morita T, Homma S. Effect of inhaled Nacetylcysteine monotherapy on lung function and redox balance in idiopathic pulmonary fibrosis. Respir Investig. 2016;54(3):170-8.

65. Kinnula VL, Hodgson UA, Lakari EK, Tan RJ, Sormunen RT, Soini YM, et al. Extracellular superoxide dismutase has a highly specific localization in idiopathic pulmonary fibrosis/usual interstitial pneumonia. Histopathology. 2006;49(1):66-74.

66. Tiitto L, Kaarteenaho-Wiik R, Sormunen R, Holmgren A, Paakko P, Soini Y, et al. Expression of the thioredoxin system in interstitial lung disease. J Pathol. 2003;201(3):363-70.

67. Vuorinen K, Ohlmeier S, Lepparanta O, Salmenkivi K, Myllarniemi M, Kinnula VL. Peroxiredoxin II expression and its association with oxidative stress and cell proliferation in human idiopathic pulmonary fibrosis. J Histochem Cytochem. 2008;56(10):951-9.

68. Anathy V, Lahue KG, Chapman DG, Chia SB, Casey DT, Aboushousha R, et al. Reducing protein oxidation reverses lung fibrosis. Nature medicine. 2018.

69. Cheresh P, Kim SJ, Tulasiram S, Kamp DW. Oxidative stress and pulmonary fibrosis. Biochim Biophys Acta. 2013;1832(7):1028-40.

70. Singh S, Vrishni S, Singh BK, Rahman I, Kakkar P. Nrf2-ARE stress response mechanism: a control point in oxidative stress-mediated dysfunctions and chronic inflammatory diseases. Free radical research. 2010;44(11):1267-88.

71. Kikuchi N, Ishii Y, Morishima Y, Yageta Y, Haraguchi N, Itoh K, et al. Nrf2 protects against pulmonary fibrosis by regulating the lung oxidant level and Th1/Th2 balance. Respir Res. 2010;11:31. 72. Reddy NM, Kleeberger SR, Cho HY, Yamamoto M, Kensler TW, Biswal S, et al. Deficiency in Nrf2-GSH signaling impairs type II cell growth and enhances sensitivity to oxidants. Am J Respir Cell Mol Biol. 2007;37(1):3-8.

73. Gonzalez-Gonzalez FJ, Chandel NS, Jain M, Budinger GRS. Reactive oxygen species as signaling molecules in the development of lung fibrosis. Transl Res. 2017;190:61-8.

74. Brand MD. Mitochondrial generation of superoxide and hydrogen peroxide as the source of mitochondrial redox signaling. Free Radic Biol Med. 2016;100:14-31.

75. Cloonan SM, Choi AM. Mitochondria in lung disease. J Clin Invest. 2016;126(3):809-20.

76. Liu X, Chen Z. The pathophysiological role of mitochondrial oxidative stress in lung diseases. J TransI Med. 2017;15(1):207.

77. Chauhan A, Vera J, Wolkenhauer O. The systems biology of mitochondrial fission and fusion and implications for disease and aging. Biogerontology. 2014;15(1):1-12.

78. Gomes LC, Di Benedetto G, Scorrano L. During autophagy mitochondria elongate, are spared from degradation and sustain cell viability. Nat Cell Biol. 2011;13(5):589-98.

79. Zank DC, Bueno M, Mora AL, Rojas M. Idiopathic Pulmonary Fibrosis: Aging, Mitochondrial Dysfunction, and Cellular Bioenergetics. Front Med (Lausanne). 2018;5:10.

80. Zandalinas SI, Mittler R. ROS-induced ROS release in plant and animal cells. Free Radic Biol Med. 2017.

81. Hecker L, Cheng J, Thannickal VJ. Targeting NOX enzymes in pulmonary fibrosis. Cell Mol Life Sci. 2012;69(14):2365-71. 
82. van der Vliet A. NADPH oxidases in lung biology and pathology: host defense enzymes, and more. Free Radic Biol Med. 2008;44(6):938-55.

83. Bernard K, Hecker L, Luckhardt TR, Cheng G, Thannickal VJ. NADPH oxidases in lung health and disease. Antioxidants \& redox signaling. 2014;20(17):2838-53.

84. Choi SH, Kim M, Lee HJ, Kim EH, Kim CH, Lee YJ. Effects of NOX1 on fibroblastic changes of endothelial cells in radiationinduced pulmonary fibrosis. Mol Med Rep. 2016;13(5):4135-42.

85. Lin C, Zhao X, Sun D, Zhang L, Fang W, Zhu T, et al. Transcriptional activation of follistatin by Nrf2 protects pulmonary epithelial cells against silica nanoparticle-induced oxidative stress. Sci Rep. 2016;6:21133.

86. Sun $B$, Wang $X$, Ji Z, Wang $M$, Liao YP, Chang CH, et al. NADPH Oxidase-Dependent NLRP3 Inflammasome Activation and its Important Role in Lung Fibrosis by Multiwalled Carbon Nanotubes. Small. 2015;11(17):2087-97.

87. Manoury B, Nenan S, Leclerc O, Guenon I, Boichot E, Planquois JM, et al. The absence of reactive oxygen species production protects mice against bleomycin-induced pulmonary fibrosis. Respir Res. 2005;6:11.

88. Carnesecchi S, Deffert C, Donati Y, Basset O, Hinz B, Preynat-Seauve O, et al. A key role for NOX4 in epithelial cell death during development of lung fibrosis. Antioxidants \& redox signaling. 2011;15(3):607-19.

89. Hecker L, Vittal R, Jones T, Jagirdar R, Luckhardt TR, Horowitz JC, et al. NADPH oxidase-4 mediates myofibroblast activation and fibrogenic responses to lung injury. Nat Med. 2009;15(9):1077-81.

90. Pache JC, Carnesecchi S, Deffert C, Donati Y, Herrmann FR, Barazzone-Argiroffo C, et al. NOX-4 is expressed in thickened pulmonary arteries in idiopathic pulmonary fibrosis. Nat Med. 2011;17(1):31-2; author reply 2-3.

91. Martyn KD, Frederick LM, von Loehneysen K, Dinauer MC, Knaus UG. Functional analysis of Nox4 reveals unique characteristics compared to other NADPH oxidases. Cell Signal. 2006;18(1):69-

82.

92. Lambeth JD. NOX enzymes and the biology of reactive oxygen. Nat Rev Immunol. 2004;4(3):181-9.

93. Ray PD, Huang BW, Tsuji Y. Reactive oxygen species (ROS) homeostasis and redox regulation in cellular signaling. Cell Signal. 2012;24(5):981-90.

94. Jones DP, Sies H. The Redox Code. Antioxidants \& redox signaling. 2015;23(9):734-46.

95. Holmstrom KM, Finkel T. Cellular mechanisms and physiological consequences of redoxdependent signalling. Nat Rev Mol Cell Biol. 2014;15(6):411-21.

96. Gupta V, Carroll KS. Sulfenic acid chemistry, detection and cellular lifetime. Biochim Biophys Acta. 2014;1840(2):847-75.

97. Hill BG, Bhatnagar A. Protein S-glutathiolation: redox-sensitive regulation of protein function. J Mol Cell Cardiol. 2012;52(3):559-67.

98. Holmgren A, Johansson C, Berndt C, Lonn ME, Hudemann C, Lillig CH. Thiol redox control via thioredoxin and glutaredoxin systems. Biochem Soc Trans. 2005;33(Pt 6):1375-7.

99. Thomas SM, Brugge JS. Cellular functions regulated by Src family kinases. Annu Rev Cell Dev Biol. 1997;13:513-609.

100. Giannoni E, Taddei ML, Chiarugi P. Src redox regulation: again in the front line. Free Radic Biol Med. 2010;49(4):516-27.

101. Roskoski R, Jr. Src protein-tyrosine kinase structure, mechanism, and small molecule inhibitors. Pharmacol Res. 2015;94:9-25.

102. Thomas SM, Brugge JS. Cellular functions regulated by Src family kinases. Annu Rev Cell Dev Biol. 1997;13:513-609.

103. Grimminger F, Gunther A, Vancheri C. The role of tyrosine kinases in the pathogenesis of idiopathic pulmonary fibrosis. Eur Respir J. 2015;45(5):1426-33. 
104. Hu M, Che P, Han X, Cai GQ, Liu G, Antony V, et al. Therapeutic targeting of SRC kinase in myofibroblast differentiation and pulmonary fibrosis. J Pharmacol Exp Ther. 2014;351(1):87-95. 105. Xie YG, Yu Y, Hou LK, Wang X, Zhang B, Cao XC. FYN promotes breast cancer progression through epithelial-mesenchymal transition. Oncol Rep. 2016;36(2):1000-6.

106. Cicchini C, Laudadio I, Citarella F, Corazzari M, Steindler C, Conigliaro A, et al. TGFbetainduced EMT requires focal adhesion kinase (FAK) signaling. Exp Cell Res. 2008;314(1):143-52.

107. Lu YY, Zhao XK, Yu L, Qi F, Zhai B, Gao CQ, et al. Interaction of Src and Alpha-V Integrin Regulates Fibroblast Migration and Modulates Lung Fibrosis in A Preclinical Model of Lung Fibrosis. Sci Rep. 2017;7:46357.

108. Boggon TJ, Eck MJ. Structure and regulation of Src family kinases. Oncogene. 2004;23(48):7918-27.

109. Zhang H, Davies KJ, Forman HJ. TGFbeta1 rapidly activates Src through a non-canonical redox signaling mechanism. Arch Biochem Biophys. 2015;568:1-7.

110. Harrison SC. Variation on an Src-like theme. Cell. 2003;112(6):737-40.

111. Lewandrowski U, Tibaldi E, Cesaro L, Brunati AM, Toninello A, Sickmann A, et al. Analysis of tyrosine-phosphorylated proteins in rat brain mitochondria. Methods Enzymol. 2009;457:117-36.

112. Salvi M, Brunati AM, Toninello A. Tyrosine phosphorylation in mitochondria: a new frontier in mitochondrial signaling. Free Radic Biol Med. 2005;38(10):1267-77.

113. Hofer A, Wenz T. Post-translational modification of mitochondria as a novel mode of regulation. Exp Gerontol. 2014;56:202-20.

114. Koc EC, Miller-Lee JL, Koc H. Fyn kinase regulates translation in mammalian mitochondria. Biochim Biophys Acta Gen Subj. 2017;1861(3):533-40.

115. Matsushima S, Kuroda J, Zhai P, Liu T, Ikeda S, Nagarajan N, et al. Tyrosine kinase FYN negatively regulates NOX4 in cardiac remodeling. The Journal of clinical investigation. 2016;126(9):3403-16.

116. MedicalEncyclopedia. Interstitial lung disease Atlanta (GA): A.D.A.M., Inc.; 2005 [updated 10-01-2018. Available from: https://medlineplus.gov/ency/article/000128.htm.

117. Kolb M, Bonella F, Wollin L. Therapeutic targets in idiopathic pulmonary fibrosis. Respiratory medicine. 2017;131:49-57.

118. Molina-Molina M, Agusti A, Crestani B, Schwartz DA, Konigshoff M, Chambers RC, et al. Towards a global initiative for fibrosis treatment (GIFT). ERJ Open Res. 2017;3(4).

119. Sugiura H, Ichikawa T, Liu X, Kobayashi T, Wang XQ, Kawasaki S, et al. N-acetyl-L-cysteine inhibits TGF-beta1-induced profibrotic responses in fibroblasts. Pulm Pharmacol Ther.

2009;22(6):487-91.

120. Hagiwara SI, Ishii Y, Kitamura S. Aerosolized administration of $\mathrm{N}$-acetylcysteine attenuates lung fibrosis induced by bleomycin in mice. Am J Respir Crit Care Med. 2000;162(1):225-31.

121. Cortijo J, Cerda-Nicolas M, Serrano A, Bioque G, Estrela JM, Santangelo F, et al. Attenuation by oral $\mathrm{N}$-acetylcysteine of bleomycin-induced lung injury in rats. Eur Respir J. 2001;17(6):1228-35.

122. Idiopathic Pulmonary Fibrosis Clinical Research N, Martinez FJ, de Andrade JA, Anstrom KJ, King TE, Jr., Raghu G. Randomized trial of acetylcysteine in idiopathic pulmonary fibrosis. N Engl J Med. 2014;370(22):2093-101.

123. Rogliani P, Calzetta L, Cavalli F, Matera MG, Cazzola M. Pirfenidone, nintedanib and Nacetylcysteine for the treatment of idiopathic pulmonary fibrosis: A systematic review and metaanalysis. Pulm Pharmacol Ther. 2016;40:95-103.

124. Myllarniemi M, Kaarteenaho R. Pharmacological treatment of idiopathic pulmonary fibrosis - preclinical and clinical studies of pirfenidone, nintedanib, and $\mathrm{N}$-acetylcysteine. Eur Clin Respir J. 2015;2. 
125. Parasassi T, Brunelli R, Costa G, De Spirito M, Krasnowska E, Lundeberg T, et al. Thiol redox transitions in cell signaling: a lesson from N-acetylcysteine. ScientificWorldJournal. 2010;10:1192-

202.

126. Gharaee-Kermani M, Moore BB, Macoska JA. Resveratrol-Mediated Repression and Reversion of Prostatic Myofibroblast Phenoconversion. PLoS One. 2016;11(7):e0158357.

127. Giovannelli L, Pitozzi V, Jacomelli M, Mulinacci N, Laurenzana A, Dolara P, et al. Protective effects of resveratrol against senescence-associated changes in cultured human fibroblasts. J Gerontol A Biol Sci Med Sci. 2011;66(1):9-18.

128. Nakamura T, Matsushima M, Hayashi Y, Shibasaki M, Imaizumi K, Hashimoto N, et al. Attenuation of transforming growth factor-beta-stimulated collagen production in fibroblasts by quercetin-induced heme oxygenase-1. Am J Respir Cell Mol Biol. 2011;44(5):614-20.

129. Verma R, Kushwah L, Gohel D, Patel M, Marvania T, Balakrishnan S. Evaluating the Ameliorative Potential of Quercetin against the Bleomycin-Induced Pulmonary Fibrosis in Wistar Rats. Pulm Med. 2013;2013:921724.

130. He X, Wang L, Szklarz G, Bi Y, Ma Q. Resveratrol inhibits paraquat-induced oxidative stress and fibrogenic response by activating the nuclear factor erythroid 2-related factor 2 pathway. J Pharmacol Exp Ther. 2012;342(1):81-90.

131. Sener G, Topaloglu N, Sehirli AO, Ercan F, Gedik N. Resveratrol alleviates bleomycininduced lung injury in rats. Pulm Pharmacol Ther. 2007;20(6):642-9.

132. Akgedik R, Akgedik S, Karamanli H, Uysal S, Bozkurt B, Ozol D, et al. Effect of resveratrol on treatment of bleomycin-induced pulmonary fibrosis in rats. Inflammation. 2012;35(5):1732-41.

133. Hertog MG, Hollman PC, Katan MB, Kromhout D. Intake of potentially anticarcinogenic flavonoids and their determinants in adults in The Netherlands. Nutr Cancer. 1993;20(1):21-9. 134. Heim KE, Tagliaferro AR, Bobilya DJ. Flavonoid antioxidants: chemistry, metabolism and structure-activity relationships. J Nutr Biochem. 2002;13(10):572-84.

135. Chen YT, Zheng RL, Jia ZJ, Ju Y. Flavonoids as superoxide scavengers and antioxidants. Free Radic Biol Med. 1990;9(1):19-21.

136. Boots AW, Haenen GR, Bast A. Health effects of quercetin: from antioxidant to nutraceutical. Eur J Pharmacol. 2008;585(2-3):325-37.

137. Nair MP, Mahajan S, Reynolds JL, Aalinkeel R, Nair H, Schwartz SA, et al. The flavonoid quercetin inhibits proinflammatory cytokine (tumor necrosis factor alpha) gene expression in normal peripheral blood mononuclear cells via modulation of the NF-kappa beta system. Clin Vaccine Immunol. 2006;13(3):319-28.

138. Gulati N, Laudet B, Zohrabian VM, Murali R, Jhanwar-Uniyal M. The antiproliferative effect of Quercetin in cancer cells is mediated via inhibition of the PI3K-Akt/PKB pathway. Anticancer Res. 2006;26(2A):1177-81.

139. Huang RY, Yu YL, Cheng WC, OuYang CN, Fu E, Chu CL. Immunosuppressive effect of quercetin on dendritic cell activation and function. J Immunol. 2010;184(12):6815-21.

140. Cao Y, Hu J, Sui J, Jiang L, Cong Y, Ren G. Quercetin is able to alleviate TGF-beta-induced fibrosis in renal tubular epithelial cells by suppressing miR-21. Exp Ther Med. 2018;16(3):2442-8.

141. Arredondo F, Echeverry C, Abin-Carriquiry JA, Blasina F, Antunez K, Jones DP, et al. After cellular internalization, quercetin causes Nrf2 nuclear translocation, increases glutathione levels, and prevents neuronal death against an oxidative insult. Free Radic Biol Med. 2010;49(5):738-47. 142. Hohmann MS, Habiel DM, Coelho AL, Verri WA, Jr., Hogaboam CM. Quercetin Enhances Ligand-induced Apoptosis in Senescent Idiopathic Pulmonary Fibrosis Fibroblasts and Reduces Lung Fibrosis In Vivo. Am J Respir Cell Mol Biol. 2019;60(1):28-40.

143. Williams RJ, Spencer JP, Rice-Evans C. Flavonoids: antioxidants or signalling molecules? Free Radic Biol Med. 2004;36(7):838-49. 
144. Navarro-Nunez L, Lozano ML, Martinez C, Vicente V, Rivera J. Effect of quercetin on platelet spreading on collagen and fibrinogen and on multiple platelet kinases. Fitoterapia. 2010;81(2):75-80. 



\section{Chapter 2 \\ Redox imbalance in IPF: a role for oxidant crosstalk between NOX enzymes and mitochondria}

Carmen Veith, Agnes W. Boots, Musa Idris, Frederik-Jan van Schooten and Albert van der Vliet.

Antioxidant Redox Signaling 2019, doi: 10.1089/ars.2019.7742. 


\section{Abstract}

Significance: Idiopathic pulmonary fibrosis (IPF) is a progressive age-related lung disease with a median survival of only 3 years after diagnosis. The pathogenic mechanisms behind IPF are not clearly understood and current therapeutic approaches have not been successful in improving disease outcomes. Recent advances: IPF is characterized by increased production of reactive oxygen species (ROS), primarily by NADPH oxidases (NOXes) and mitochondria, as well as altered antioxidant defenses. Recent studies have identified the NOX isoform NOX4 as a key player in various important aspects of IPF pathology. In addition, mitochondrial dysfunction is thought to enhance pathological features of IPF, in part by increasing mitochondrial ROS production and altering cellular metabolism. Recent findings indicate reciprocal interactions between NOX enzymes and mitochondria, which affect regulation of NOX activity as well as mitochondrial function and $\mathrm{mtROS}$ production, and collectively promote epithelial injury and profibrotic signaling. Critical Issues and Future Directions: The precise molecular mechanisms by which ROS from NOX or mitochondria contribute to IPF pathology are not known. This review will summarize current knowledge with respect to the various aspects of ROS imbalance in the context of IPF and its proposed roles in disease development, with specific emphasis on the importance of inappropriate NOX activation, mitochondrial dysfunction, and the emerging evidence of NOX-mitochondria crosstalk as important drivers in IPF pathobiology.

Keywords: IPF; reactive oxygen species; aging; lung; NOX4; mitochondria 


\section{Introduction}

Idiopathic pulmonary fibrosis (IPF) is a progressive and irreversible lung disease of unknown etiology, and represents a specific form of chronic fibrosing interstitial pneumonia that occurs primarily in the lung. The pathogenic mechanisms of IPF are still largely unclear, but over the last decade, the paradigm of IPF pathogenesis has shifted from a generally inflammation-driven disease to an epithelial-fibroblastic one, resulting from a disrupted homeostasis of epithelial cells upon damage by various triggers $(1,2)$. Continuous epithelial injury results in aberrant wound healing responses and eventually causes excessive collagen deposition in the alveolar epithelium and remodeling of the lung structure (1). IPF is generally diagnosed in the sixth decade of life, and aging is now recognized as one of the strongest risk factors for IPF (3). As the average life expectancy continues to increase worldwide, the incidence of age-related lung diseases such as IPF will also increase at a rapid pace. It is estimated that the number of people over 60 years of age will increase by almost 50\% between 2015 and 2050, and account for 22\% of the total world population (4). As the elderly population is growing, there is a growing need to understand the underlying mechanisms of aging and age-associated phenomena and their contribution to disease pathogenesis. Aging results in decreased resistance to multiple forms of stress and progressive loss of regenerative capacity, and thereby increases susceptibility to chronic lung diseases including IPF, chronic obstructive pulmonary disease (COPD), and lung cancer (5). Indeed, common hallmarks of aging, including genomic instability, telomere shortening, epigenetic alterations, loss of proteostasis, dysregulated nutrient sensing, mitochondrial dysfunction, cellular senescence/apoptosis, stem cell exhaustion, and distorted intercellular communication (6), are often also seen in IPF and frequently occur prematurely (5, 7-13).

Both aging and fibrotic diseases are associated with an increased oxidant burden (14-16) and lung tissues as well as breath condensates from IPF patients show increased levels of oxidative damage markers such as 8-isoprostane and carbonylated proteins (17-19). Studies in mice indicate that aging increases susceptibility to pulmonary fibrosis, likely due to decreased resistance to oxidative stress (20). The main cellular sources of reactive oxygen species (ROS) are NADPH oxidases (NOXes) and mitochondria (21), and while ROS production from both sources serves various biological roles in e.g. host defense, cell differentiation, or cellular responses to injury $(22,23)$, both have also been implicated in IPF pathology. For example, various lines of evidence indicate that mitochondria are dysfunctional in IPF (24), resulting in increased production of mitochondrial ROS (mtROS) (12), and that approaches to attenuate mtROS may be beneficial (25). In addition, recent studies have implicated NOX4 as an important player in the development of IPF (26), based on its ability to induce alveolar epithelial cell (AEC) death, (myo)fibroblast differentiation and collagen deposition (27). More recently, NOX4 has also been 
implicated as a mediator of mitochondrial dysfunction (28), suggesting cross-talk between these two ROS-generating systems. This review will summarize our current knowledge with respect to the presence of a redox imbalance in IPF, and the importance of ROS in the development and progression of IPF. Specifically, we will highlight the importance of NOX4 and the emerging crosstalk between NOX4 and mitochondria. Finally, we will discuss important gaps of knowledge and potential future approaches for development of more effective treatment strategies. 


\section{Idiopathic pulmonary fibrosis - pathology and affected cell types}

\subsection{General pathology}

IPF is the most common form of idiopathic interstitial lung diseases and is characterized by scarring of the lung tissue causing symptoms such as non-productive cough and breathlessness. As a chronic progressive lung disease, IPF has a median survival of 2-4 years after diagnosis due to respiratory failure and hypoxemia (29). Two thirds of IPF patients are older than 60 years at time of diagnosis (30), and aging is the most wellknown risk factor for developing this debilitating disease. Although it is a relatively rare disease that currently affects approximately 5 million people worldwide, its overall incidence increases every year probably due to the increasing number of elderly individuals $(31,32)$. In the United States, the incidence of IPF varies between 1.1 new cases per 100.000 person-years in 18-34 old individuals up to 19.3 in individuals older than 55 of age (33). Besides aging, IPF has several other risk factors including exposure to environmental factors, e.g. cigarette smoke and asbestos (34). Additionally, the pathogenesis of the disease is associated with genomic instability, mitochondrial dysfunction and altered intracellular signaling (6), all hallmarks of normal aging but also all processes compromised by environmental factors such as tobacco smoking.

Histologically, the most prominent morphological hallmark of IPF is usual interstitial pneumonia (UIP). One histologic feature of UIP is honeycombing characterized by clusters of cysts, often filled with mucus, in a subpleural location (35). An additional feature of UIP is the presence of fibroblastic foci. Fibroblastic foci contain granuloma of activated myofibroblasts producing extracellular matrix (ECM) proteins and are mainly found in dense fibrotic areas of the lungs (36). UIP is further characterized by hyperplastic type II AECs and reduced type I AECs. During IPF development, a disturbed lung homeostasis results in a profibrotic milieu thereby affecting the survival and death of both fibroblasts and AECs. AECs become more prone to undergo apoptosis (37) while fibroblasts and myofibroblasts become more resistant against this process (38). Also, distinct alterations in immune cells are reported in IPF as the lungs of these patients exhibit chronic inflammation associated with immunosenescence (39).

\subsection{Cell-specific aspects of IPF}

IPF is a complex disease which involves the contribution of many different cell types. The following paragraphs will summarize the specific involvement of distinct lung cell types, such as epithelial cells, fibroblasts, immune cells and endothelial cells. 


\section{Epithelial cells}

The alveolar lining of the lungs is covered by $95 \%$ type I AECs, important in facilitating gas exchange, and $5 \%$ type II AECs that are involved in surfactant production. In IPF pathogenesis, repetitive injury to the alveolar epithelium leads to abnormal reepithelialization and repair of AECs (40). Lung tissue from IPF patients typically shows loss of integrity of the alveolar epithelium with disruption of basement membrane integrity and collapse of the alveolar structure. While type II AECs normally proliferate and differentiate into type I AECs in response to injury to promote re-epithelialization, in the context of fibrosis, AECs are more prone to undergo apoptosis which is a prominent feature during IPF manifestation. Indeed, AEC type II cells stain positive for apoptotic markers in patients with IPF compared to controls (41). Moreover, experimental induction of epithelial cell apoptosis is sufficient to promote features of fibrosis (37), and inhibition of apoptosis attenuates bleomycin-induced pulmonary fibrosis $(42,43)$. In addition to evidence for AEC type II apoptosis in IPF, numbers of type I AEC in the lung of IPF patients are reduced causing $A E C$ type II to undergo hyperplasia thereby replacing type I AEC and inducing ineffective re-epithelialization (44). Additionally, AEC secrete a variety of profibrotic mediators, such as platelet-derived growth factor (PDGF), endothelin-1, angiotensin II, connective tissue growth factor (CTGF) and transforming-growth factor (TGF) $\beta$, which can induce fibroblasts to differentiate into myofibroblasts (13). Furthermore, increased epithelial senescence results in enhanced secretion of growth factors, cytokines, chemokines and matrix metalloproteins (MMP) that stimulate continuous fibroblast activity (45). Additionally, these factors promote a senescenceassociated secretory phenotype (SASP) which is a key event in IPF possibly leading to diminished capacity of AEC regeneration (13). Finally, AECs cells have also suggested to contribute to fibroblast development in IPF due to epithelial-to-mesenchymal transition (EMT), a process in which epithelial cells lose their epithelial phenotype and acquire more mesenchymal/fibroblastic characteristics such as $\alpha$-smooth muscle actin and fibronectin expression. Indeed, epithelial cells isolated from IPF patients have been found to express not only epithelial but also mesenchymal markers (46). However, the overall significance of EMT to IPF development/manifestation is still not completely clear (47). Lineage tracing strategies have suggested a contribution for EMT to increased presence of fibroblasts in IPF lungs $(46,48,49)$, but other studies have also disputed that epithelial cells are indeed the source of fibroblasts (50). In summary, repetitive epithelial injury and dysregulated repair are critical features in IPF development and progression as they lead to increased apoptosis, abnormal regeneration, and EMT-like features. Moreover, TGF- $\beta$, an important growth factor in IPF, can promote epithelial apoptosis as well as EMT (51) further indicating the important role of the lung epithelium in IPF. 


\section{Fibroblasts}

Epithelial cells within the airways and alveoli are in close proximity to and communication with mesenchymal cells such as pulmonary fibroblasts, referred to as the epithelial mesenchymal trophic unit. Consequently, epithelial damage impacts on fibroblasts by promoting their differentiation into myofibroblasts which is mediated by TGF- $\beta 1$ (52). Myofibroblasts actively participate in remodeling of the lung by secreting extracellular matrix (ECM) proteins into the lung alveolar structure also upon induction by TGF- $\beta 1$. Various sources of myofibroblasts have been suggested to be recruited to the site of injury; including proliferation and differentiation of resident lung fibroblasts, epithelial and endothelial cells that transform into fibroblasts and transition of fibrocytes or other circulating progenitor cells (53). During normal wound repair, myofibroblasts undergo apoptosis and clearance of the ECM occurs upon closing the wound, thus avoiding the formation of scar tissue. In IPF, however, overactivation of myofibroblasts due to persistent epithelial injury, leads to aberrant wound repair (54) and increased scarring occurs which is characterized by the formation of fibroblastic foci, a major hallmark of IPF (55) and cause of decreased pulmonary function $(56,57)$. Additionally, fibroblasts and myofibroblasts from IPF patients have an apoptosis-resistant phenotype which is also associated with higher levels of senescence markers, such as p16, compared to fibroblasts from age-matched controls $(11,58)$. In summary these studies suggest that fibroblasts from patients with IPF are characterized by a senescent apoptosis-resistant phenotype and are thereby actively involved in the progression of the disease by remodeling of the lung structure.

\section{Inflammatory cells}

During the last decades, a common view has been that chronic inflammation underlies the pathogenic sequence in IPF as a main cause of lung injury and fibrogenesis. Although this "inflammatory fibrosis" hypothesis has been somewhat invalidated $(40,59)$, chronic inflammation is still considered a common feature of IPF yet most likely as a secondary event instead (40). Damaged epithelial cells release a variety of chemokines and cytokines that recruit inflammatory monocytes and neutrophils to the injured site. Recruitment and activation of neutrophils to the bronchoalveolar space have been shown to correlate with disease progression (60) and are also a predictor of early mortality in IPF (61). Normally, monocytes differentiate into phagocytic macrophages that phagocytose dead cells and neutrophils. However, because of the continuous injury in IPF, neutrophils and macrophages are not eliminated quickly enough. Consequently, they can develop resistance to apoptosis which will further exacerbate the ongoing inflammation and fibrotic cycle (51). Macrophages can mediate antifibrotic as well as profibrotic effects depending on their phenotype. Classically activated macrophages (M1) are activated by the T-helper $\left(T_{H}\right)$ cell 1 cytokine interferon (IFN) $-\gamma$ and mainly involved in the initial 
inflammatory response, whereas alternatively activated macrophages (M2) are activated by $\mathrm{T}_{\mathrm{H}} 2$ cytokines including interleukin (IL)- 4 and IL-13 and involved in tissue remodeling and resolution of inflammation (62). Furthermore, studies also have demonstrated that the $T_{H} 1 / T_{H} 2$ balance is disturbed in favor of $T_{H} 2$ cytokines $(63,64)$ further contributing to a M2 phenotype. M2 polarized macrophages promote profibrotic effects through the section of profibrotic mediators including TGF- $\beta$ (65). Additionally, alveolar neutrophils and macrophages will undergo a respiratory burst upon phagocytosis which in turn also promotes alveolar epithelial cell injury thereby inducing a vicious circle (17). Taken together, studies have proven that inflammatory cells participate in the profibrotic circle by secreting profibrotic and proinflammatory cytokines although they are not the prominent cell type involved in the development and initial manifestation of IPF.

\section{Endothelial cells}

Endothelial cells are important in maintaining the alveolar-capillary barrier for facilitating gas exchange (66). Pulmonary endothelial cells are close to the interstitium, the primary site of injury during IPF, and cover the intravascular lumen making them vulnerable to injuries. Endothelial cells from IPF patients $(67,68)$, and pulmonary endothelial cells from mice with bleomycin-induced pulmonary fibrosis, display increased markers of endothelial cell injury (69) and endothelial cell apoptosis (70), which result in loss of integrity of the alveolar-capillary barrier. Moreover, endothelial progenitor cells are reduced in IPF patients (71), which impairs effective re-endothelialization and vascular repair upon injury. Additionally, the damaged endothelium secretes a variety of factors that activate circulating platelets and von Willebrand factor, which are involved in angiogenesis and recruitment of inflammatory cells (72). Finally, endothelial cells have also been implicated as source of fibroblasts through endothelial-to-mesenchymal transition during fibrogenesis $(73,74)$. Endothelial dysfunction in patients with IPF has also been correlated with pulmonary hypertension, a common comorbidity in IPF (75).

Taken together, AEC apoptosis and (myo)fibroblast activation are now considered the primary responsible events contributing to excessive lung remodeling in IPF, whereas the specific role of endothelial cells and inflammation is less well established and still needs to be further elucidated.

\subsection{Current treatment strategies for IPF}

Currently, there are only two FDA-approved drugs available for patients with IPF. Both pirfenidone and nintedanib can slow down the progression of the disease (76), although they cannot reverse existing pulmonary fibrotic injury. Pirfenidone (Esbriet ${ }^{\oplus}$; Roche/Genentech USA,) is thought to have antioxidative, anti-inflammatory, and antifibrotic effects, although the exact working mechanisms are not clear as confirmatory data from IPF patients are lacking (76). In vitro studies and studies in bleomycin-induced 
murine pulmonary fibrosis indicate that it reduces markers of oxidative stress $(77,78)$, decreases the secretion of pro-inflammatory cytokines (e.g. TNF- $\alpha$, IL-1 $\beta$, IL-6) (79), and inhibits fibroblast proliferation, myofibroblast differentiation, and TGF- $\beta$-induced collagen production (80). Nintedanib (Ofev; Boehringer Ingelheim) is an intracellular tyrosine kinase inhibitor, which mainly inhibits the platelet-derived growth factor receptor (PDGFR), the fibroblast growth factor receptor (FGFR) and vascular endothelial growth factor receptor (VEGFR) as well as non-receptor tyrosine kinases of the SRC family (76), which mediate its antifibrotic effects through its inhibitory action on fibroblasts (81). 


\section{The disturbed redox balance in IPF}

\subsection{ROS in biology and disease}

All cells in the body consume and metabolize oxygen and as a result produce ROS, which include superoxide anion $\left(\mathrm{O}_{2}{ }^{\circ}\right)$, hydrogen peroxide $\left(\mathrm{H}_{2} \mathrm{O}_{2}\right)$, and hydroxyl radical $\left(\mathrm{OH}^{\circ}\right)$, as well as other, secondary ROS. ROS are produced as a result of aerobic metabolism within the mitochondrial electron transport chain (ETC), but can also be generated by various enzyme systems, including xanthine oxidase, lipid peroxidases, uncoupled endothelial NO synthase, cytochrome P450 enzymes, and NADPH oxidases (NOX) (82). The latter group of enzymes are the only enzymes that are considered to generate ROS as their primary product, and serve essential functions as mediators of host defense against diverse pathogens, and in various other aspects of cell biology through redox-based cell signaling $(23,83)$. Inappropriate or uncontrolled ROS production is generally thought to contribute to disease pathology, due to overproduction of ROS production by e.g. increased NOX activation or by mitochondrial dysfunction, or due to compromised metabolism of ROS by antioxidant systems (84). Such an increased oxidant burden can lead to extensive molecular damage to macromolecules including DNA, lipids and proteins (85), thereby causing protein dysfunction and altered proteostasis, genomic instability, or production of secondary reactive lipid-derived species (e.g. electrophiles such as malondialdehyde (MDA) or 4-hydroxynonenal).

Specific to IPF, several studies reported that IPF patients have a higher oxidant burden compared to healthy controls. Indeed, ROS production is dysregulated due to enhanced NOX expression and activation, as well as mitochondrial dysfunction and increased mitochondrial ROS (mtROS) generation $(17,86)$. Pulmonary inflammatory cells obtained from epithelial lining fluid (ELF) of IPF patients generate higher levels of ROS compared to those of healthy controls (87) and IPF patients have increased levels of $\mathrm{H}_{2} \mathrm{O}_{2}$ within their exhaled breath condensates (EBC) (18). IPF patients also demonstrate increased circulating markers of lipid peroxidation compared to healthy non-smokers (88), and increased levels of the lipid oxidation product 8-isoprostane within bronchoalveolar lavage fluid (BALF) and EBC (15). BALF or lung tissue from IPF patients also contains higher amounts of irreversibly oxidized proteins (e.g. carbonylation, nitration) $(19,89,90)$, and epithelial cells of patients with usual interstitial pneumonia (IP) show increased levels of DNA oxidation, illustrated by 8-hydroxy-deoxyguanosine (8-OHdG) (91). In summary, IPF patients show increased markers of oxidative damage (92). However, evidence for their causal contribution to the IPF disease is less prominent, and will be discussed in the following sections. 


\subsection{Pulmonary antioxidant defense system in IPF}

All organs, including the lung, contain a variety of antioxidant systems to prevent inappropriate ROS production or unwanted actions of cellular ROS. These include enzymes that metabolize ROS (superoxide dismutases, catalase, peroxiredoxins, glutathione peroxidases), thiol reductases that reverse oxidative cysteine modifications (thioredoxin, glutaredoxin), phase 2 detoxifying enzymes (e.g. glutathione-S-transferases), metal binding proteins (transferring, lactoferrin) and small molecular weight antioxidants (vitamins and glutathione) (84). A number of studies have demonstrated that several of these antioxidant systems are altered or impaired in IPF (Table 2.1).

Table 2.1: Alterations in antioxidant status in IPF patients.

\begin{tabular}{|c|c|c|c|c|}
\hline Antioxidant & Sample & Groups (n) & Change & Reference \\
\hline \multicolumn{5}{|l|}{$\begin{array}{l}\text { Non-enzymatic } \\
\text { antioxidants }\end{array}$} \\
\hline GSH & $\begin{array}{l}\text { ELF } \\
\text { BALF } \\
\text { Blood } \\
\text { Blood } \\
\text { Sputum/plasma } \\
\text { ELF } \\
\text { BALF } \\
\text { Plasma/BALF }\end{array}$ & $\begin{array}{l}\text { IPF (15) vs Ctrl (19) } \\
\text { IPF (23) vs Ctrl (17) } \\
\text { [smokers excluded] } \\
\text { IPF (11) vs Ctrl (9) } \\
\text { IPF (22) vs Ctrl (29) } \\
\text { IPF (16) vs Ctrl (15) } \\
\text { IPF (17) vs Ctrl (14) } \\
\text { IPF (17) vs Ctrl (14) } \\
\text { IPF (16) vs Ctrl (20) }\end{array}$ & $\begin{array}{l}\text { Decreased } \\
\text { Decreased } \\
\text { Decreased } \\
\text { Decreased } \\
\text { Decreased } \\
\text { Decreased } \\
\text { No change } \\
\text { No change }\end{array}$ & $\begin{array}{l}(93) \\
(88) \\
(95) \\
(96) \\
(97) \\
(94) \\
(94) \\
(112)\end{array}$ \\
\hline GSSG & $\begin{array}{l}\text { BALF } \\
\text { Blood } \\
\text { Blood } \\
\text { BALF } \\
\text { Plasma }\end{array}$ & $\begin{array}{l}\text { IPF (23) vs Ctrl (17) } \\
\text { [smokers excluded] } \\
\text { IPF (11) vs Ctrl (9) } \\
\text { IPF (22) vs Ctrl (29) } \\
\text { IPF (16) vs Ctrl (20) } \\
\text { IPF (16) vs Ctrl (20) }\end{array}$ & $\begin{array}{l}\text { No change } \\
\text { No change } \\
\text { Increased } \\
\text { Increased } \\
\text { No change }\end{array}$ & $\begin{array}{l}(88) \\
(95) \\
(96) \\
(112) \\
(112)\end{array}$ \\
\hline $\begin{array}{l}\text { Trolox } \\
\text { equivalent } \\
\text { antioxidant } \\
\text { capacity (TEAC) }\end{array}$ & $\begin{array}{l}\text { Plasma/BALF } \\
\text { Plasma }\end{array}$ & $\begin{array}{l}\text { IPF (23) vs Ctrl (17) } \\
\text { [smokers excluded] } \\
\text { IPF (11) vs Ctrl (9) }\end{array}$ & $\begin{array}{l}\text { Decreased } \\
\text { No change }\end{array}$ & $\begin{array}{l}(88) \\
(95)\end{array}$ \\
\hline Uric acid & $\begin{array}{l}\text { Plasma } \\
\text { Plasma/BALF }\end{array}$ & $\begin{array}{l}\text { IPF (11) vs Ctrl (9) } \\
\text { IPF (16) vs Ctrl (20) }\end{array}$ & $\begin{array}{l}\text { No change } \\
\text { Increased }\end{array}$ & $\begin{array}{l}(95) \\
(112)\end{array}$ \\
\hline Vitamin A & Plasma/BALF & IPF (16) vs Ctrl (20) & Increased & $(112)$ \\
\hline Vitamin C & $\begin{array}{l}\text { Blood } \\
\text { Plasma/BALF }\end{array}$ & $\begin{array}{l}\text { IPF (11) vs Ctrl (9) } \\
\text { IPF (16) vs Ctrl (20) }\end{array}$ & $\begin{array}{l}\text { No change } \\
\text { Increased }\end{array}$ & $\begin{array}{l}(95) \\
(112)\end{array}$ \\
\hline Vitamin E & Plasma/BALF & IPF (16) vs Ctrl (20) & Increased & (112) \\
\hline \multicolumn{5}{|l|}{$\begin{array}{l}\text { Enzymatic } \\
\text { antioxidants }\end{array}$} \\
\hline CAT & Lung tissue & IPF (12) vs Ctrl (10) & Decreased & $(106)$ \\
\hline SOD1 & Serum & IPF (25) vs Ctrl (40) & Increased & $(103)$ \\
\hline
\end{tabular}




\begin{tabular}{|l|l|l|l|l|}
\hline SOD3 & Lung tissue & $\begin{array}{l}\text { IPF (10) (fibrotic vs } \\
\text { non-fibrotic tissue) }\end{array}$ & Decreased & (99) \\
\hline GRX1 & $\begin{array}{l}\text { Lung tissue } \\
\text { Lung tissue }\end{array}$ & $\begin{array}{l}\text { IPF (160) vs Ctrl (132) } \\
\text { IPF (5) vs Ctrl (5) }\end{array}$ & $\begin{array}{l}\text { Decreased } \\
\text { Decreased }\end{array}$ & $\begin{array}{l}(110) \\
(109)\end{array}$ \\
\hline PRXII & Lung tissue & IPF (10) vs Ctrl (10) & Decreased & $(108)$ \\
\hline TRX & Lung biopsies & UIP (15) vs Ctrl (6) & Decreased & $(107)$ \\
\hline $\begin{array}{l}\text { Redox-sensitive } \\
\text { transcription } \\
\text { factor }\end{array}$ & & & & \\
\hline Nrf2 & & & & \\
& $\begin{array}{l}\text { Lung tissue } \\
\text { Lung tissue } \\
\text { [epithelium] }\end{array}$ & $\begin{array}{l}\text { IPF (16) vs Ctrl (20) } \\
\text { IPF (7) vs Ctrl (7) }\end{array}$ & $\begin{array}{l}\text { Increased } \\
\text { Increased } \\
\text { Increased }\end{array}$ & $\begin{array}{l}(112) \\
\text { (7) vs Ctrl (7) }\end{array}$ \\
\hline
\end{tabular}

Among the earliest lines of evidence of altered antioxidant status in IPF are findings of reduced levels of the cellular antioxidant thiol-containing tripeptide glutathione (GSH) within the epithelial lining fluid (ELF) of the lower respiratory tract $(93,94)$ as well as in blood $(95,96)$ and sputum $(97)$. In some cases, these decreases were accompanied by increased levels of the oxidized form of GSH, glutathione disulfide (GSSG), thus illustrating an altered redox balance in the alveolar lumen of IPF patients. Of the three known isoforms of superoxide dismutase (SOD), which catalyzes the dismutation of $\mathrm{O}_{2}{ }^{-{ }^{-}}$into $\mathrm{H}_{2} \mathrm{O}_{2}$ and $\mathrm{O}_{2}$ (98), some studies suggest that lung tissue expression of extracellular SOD (SOD3) is reduced in IPF (Table 1) (99). The importance for IPF pathology is supported by studies using SOD3 knockout mice, which were found to have greater pulmonary fibrosis in response to bleomycin compared to wildtype littermates (100) and, conversely, overexpression of SOD3 protects mice from developing pulmonary fibrosis (101). Most likely SOD3 prevents ROS-induced ECM degradation (102). The role of SOD1 and SOD2 in the progression of IPF is less well understood. In fact, SOD1 has been reported to be increased in IPF patients (103) and SOD1 knockout mice developed less oxidative stress and were protected from asbestos-induced pulmonary fibrosis compared to wildtype littermates (104). The expression of catalase, an important scavenger of $\mathrm{H}_{2} \mathrm{O}_{2}$ that is widely expressed within the alveolar epithelium as well as the inflammatory cells in the lung, was found to be attenuated by TGF- $\beta 1$ (105) and is also decreased in lungs of IPF patients (106). Since catalase is capable of inhibiting $\mathrm{H}_{2} \mathrm{O}_{2}$-mediated activation of fibroblasts (102), such a decrease in catalase may contribute to $\mathrm{H}_{2} \mathrm{O}_{2}$-mediated fibroblast activation in IPF. Additional studies also indicate alterations in other redox proteins in IPF, such as thioredoxins (TRX), peroxiredoxins (PRX) and glutaredoxins (GRX). For example, thioredoxin (TRX) is decreased in the alveolar epithelium of patients with UIP compared to controls but is increased in the metaplastic alveolar epithelium (107). Also, PRXII is increased in the hyperplastic epithelium of IPF patients but is decreased in IPF lung tissue compared to controls (108). Moreover, it has been shown that GRX1 mRNA expression as 
well as enzymatic activity is decreased in patients with IPF compared to non-IPF individuals, and recent studies indicate that GRX1 activity is lost in patients with IPF primarily by oxidative inactivation $(109,110)$. Moreover, consistent with a role for GRX1 in reversing protein S-glutationylation, observed decreases in GRX are accompanied with an increase in protein S-glutathionylation (PSSG), and were found to correlate significantly with reduced lung function in IPF patients (110). Taken together, while several studies highlighted reduced levels of activity of antioxidant systems in IPF, such changes appear to be highly variable due to the heterogeneous nature of IPF pathology. Hence, the specific contributions of such alterations to IPF development are not always clear.

Many antioxidant defense systems are under transcriptional control by the redox sensitive transcription factor nuclear factor erythroid 2 related factor 2 (Nrf2) (21). This factor binds to antioxidant response element (ARE) in the nucleus to induce expression of e.g. hemeoxygenase 1 (HO-1), glutathione-S-transferase (GST), NADP(H) quinone oxidoreductase 1 (NQO1), thioredoxin and $\gamma$-glutamylcysteine synthetase (GCS), indicating that these systems are often induced in response to oxidative stress (111). Two clinical studies have shown increased Nrf2 expression in lungs of IPF patients $(112,113)$ with one of them displaying increased levels in the hyperplastic alveolar epithelium, yet not fibroblastic foci, of IPF patients compared to their normal epithelium as well as that of healthy controls (113). Animal studies support the importance of Nrf2 in IPF development, since AECs isolated from Nrf2-deficient mice are more prone to oxidant-induced cell death (114) and Nrf2-deficient mice develop more fibrosis in response to bleomycin (115). It is important to recognize, however, that Nrf2 upregulation does not necessarily lead to Nrf2 activation and induction of antioxidant gene expression. Indeed activation of Nrf2-induced antioxidant responses are declined with increasing age $(116,117)$, which is associated with simultaneous upregulation of negative Nrf2 regulators such as $\mathrm{c}-\mathrm{MYC}$ and $\mathrm{BACH}-1$ (118). Hence, upregulation of Nrf2 in the context of IPF may not necessarily lead to enhanced activation, and therefore enhance susceptibility to oxidative stress.

Taken together, these results suggest that the activation of Nrf2 is involved in fibrosis, but its upregulation alone is not enough to counteract the increased ROS associated with the pathophysiology of this disease.

\subsection{Contributions of disturbed redox homeostasis to IPF pathology}

The combination of increased ROS production and compromised antioxidant mechanisms in IPF would suggest that dysregulated redox processes may contribute to the pathology of IPF. However, the molecular mechanisms by which such dysregulated redox processes contribute to IPF pathology are not fully elucidated. Over the past decade, it has become widely appreciated that biological ROS production serves a broad range of physiological functions through redox-dependent signaling processes that control proliferation, migration, differentiation or survival, by inducing specific and reversible redox-mediated 
post-translational modifications on redox-sensitive proteins $(119,120)$. Consequently, IPF pathology may be mediated by dysregulated redox processes rather than "oxidative stress" per se (121).

One commonly accepted mechanism by which increased ROS production in IPF contributes to disease pathology is by promoting AEC death (91) and the highly aberrant wound healing response after chronic repetitive injury to the lung epithelium (122). Extracellular generation of $\mathrm{H}_{2} \mathrm{O}_{2}$ by lung myofibroblasts may mediate additional fibrogenic effects by inducing apoptosis of adjacent lung epithelial cells (123). One mechanism of apoptotic cell death involves activation of the death receptor Fas by Fas ligand (FasL), which contributes to cell death by caspase activation (124). Myofibroblasts derived from IPF patients are capable of inducing apoptosis of alveolar epithelial cells through Fasdependent mechanisms, which is enhanced by oxidative modification of the Fas-receptor through S-glutathionylation on cysteine residue $294(125,126)$. The increased oxidant burden also results in myofibroblast accumulation with an apoptosis resistant phenotype (58), which is linked with impaired induction of $\mathrm{Nrf2}$ and increased $\mathrm{H}_{2} \mathrm{O}_{2}$ production (20). ROS play a critical role in the activation of the profibrotic cytokine TGF- $\beta$. TGF- $\beta 1$ is involved in epithelial cell apoptosis, EMT, epithelial cell migration, fibroblast proliferation and differentiation as well as myofibroblast activation (127). TGF- $\beta$ is synthesized as an inactive precursor bound to latency-binding peptide (LAP) and secreted as a latent form, which can be activated by ROS through disruption of its interaction with LAP (128). In vitro studies have shown that ROS can increase the release of TGF- $\beta$ from alveolar epithelial cells (129). In turn, TGF- $\beta$ also increases ROS production through mitochondria and NOXes in addition to suppressing antioxidant systems (130), introducing a vicious cycle between ROS and TGF- $\beta$ further contributing to a fibrotic milieu. ROS production also contributes to chronic inflammation through the activation of nuclear-factor-kappa B (NF-kB), which in turn induces expression of various pro-inflammatory cytokines (102).

In summary, the dysregulated redox balance in IPF may contributes to disease progression by diverse and interrelated mechanisms, including apoptotic AECI cell death, myofibroblast activation, and inflammation (Figure 2.1). To appreciate the specific roles of ROS-based mechanisms in IPF, it is important to consider the major sources of dysregulated ROS production, which may include inappropriate activation of NOX enzymes or mitochondrial ROS production (82). The remainder of this review will discuss current knowledge with respect to their specific roles in IPF pathobiology. 

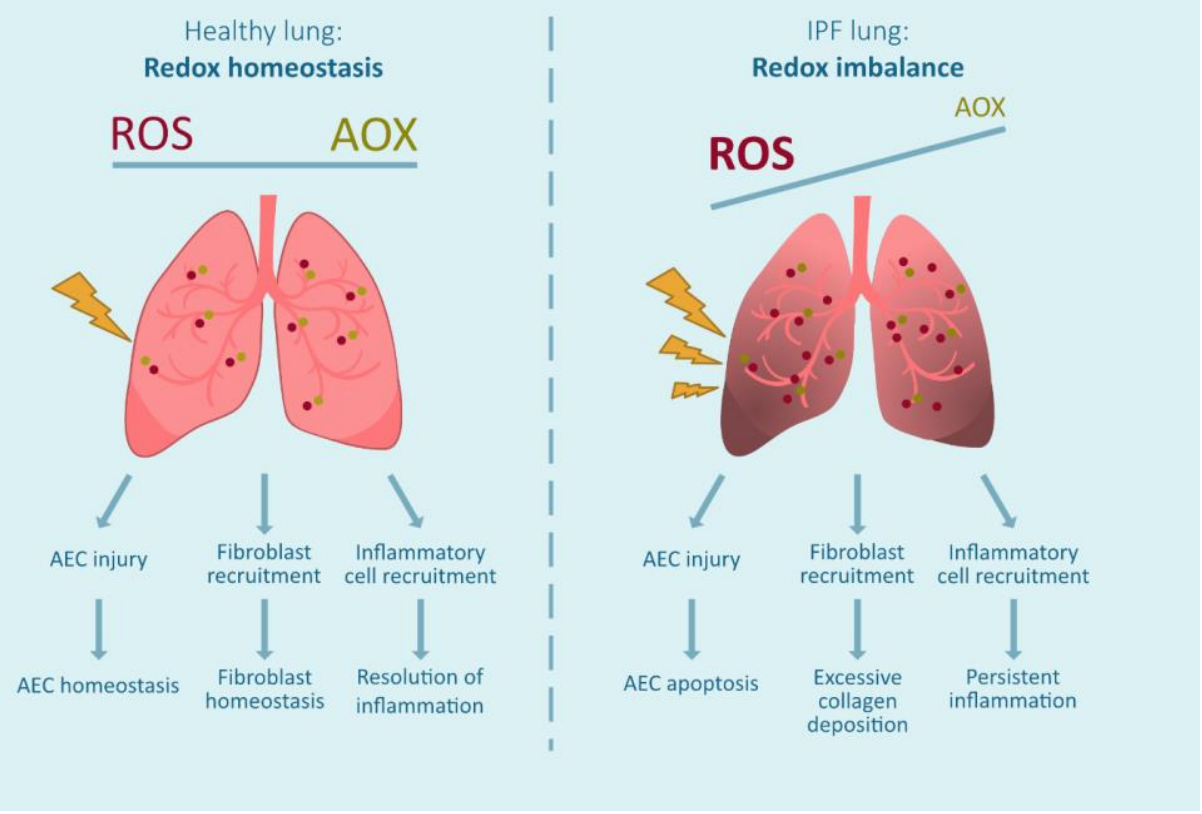

Figure 2.1: Altered lung redox homeostasis in IPF. In a healthy lung there is a redox homeostasis, e.g. ROS produced by exogenous or endogenous sources (mitochondria, NADPH oxidases, inflammatory cells) are appropriately countered by antioxidants (AOX). In IPF, there is a redox imbalance as ROS-generating processes are enhanced (increased NOX, mitochondrial dysfunction) and some antioxidant systems are compromised. This redox imbalance is thought to contribute to epithelial cell death, excessive collagen deposition, and persistent inflammation, resulting in pulmonary fibrosis and tissue scarring. 


\section{NOX enzymes in the pathophysiology of IPF}

\subsection{The NOX family}

The NADPH-oxidase (NOX) family consists of seven NOX homologs, NOX1-5 and the dual oxidases DUOX1 and 2 (Figure 2.2). All NOX isoforms have six trans-membrane spanning alpha helices with cytosolic $\mathrm{N}$ and $\mathrm{C}$-termini. The $\mathrm{C}$-terminal flavoprotein domain contains an NADPH-binding region and a flavin adenine dinucleotide binding region, whereas the $\mathrm{N}$-terminal hydrophobic domain consists of six transmembrane alpha helices that contain two heme-binding sites (131). Through the membrane-associated flavocytochrome $b_{558}$ (gp91 ${ }^{\text {phox }}$ ) and $\mathrm{p} 22^{\text {phox }}$ as well as various cytosolic cofactors, active NOX enzymes promote transmembrane electron transfer from NADPH to $\mathrm{O}_{2}$ thereby reducing it to superoxide $\left(\mathrm{O}_{2}{ }^{\circ-}\right)$ and hydrogen peroxide $\left(\mathrm{H}_{2} \mathrm{O}_{2}\right)$ (83). Activation of NOX1-3 requires association with p22 $2^{\text {phox }}$ as well as assembly with Rac-GTPase subunits and cytosolic activation proteins, for instance $\mathrm{p} 47^{\text {phox }}$ and $\mathrm{p} 67^{\text {phox }}$ or their homologs NOX organizer 1 (NOXO1) and NOX activator 1 (NOXA1) (83). NOX4 also needs association with p22 $2^{\text {phox }}$ but does not require any other cofactors for its activation and is thought to be constitutively active (132). In contrast, NOX5, DUOX1 and DUOX2 are activated by calcium signaling and binding to their calcium-binding EF-hand domains and do not need $\mathrm{p} 22^{\text {phox }}$ or other co-factors for their activation (133). DUOX1 and DUOX2 also contain an extracellular peroxidase domain but its exact function in mammalian enzymes is still unclear (134). The NOX homologs are differentially expressed and regulated in various tissues and have different subcellular localizations and even produce distinct ROS. NOX1-3 and NOX5 primarily produce $\mathrm{O}_{2}{ }^{*}$ whereas NOX4, DUOX1 and DUOX2 mainly produce $\mathrm{H}_{2} \mathrm{O}_{2}$ (135), and through their production of ROS, the various NOX enzymes regulate host defense, but also cell proliferation, differentiation and migration, by redox-dependent signaling pathways (136). 

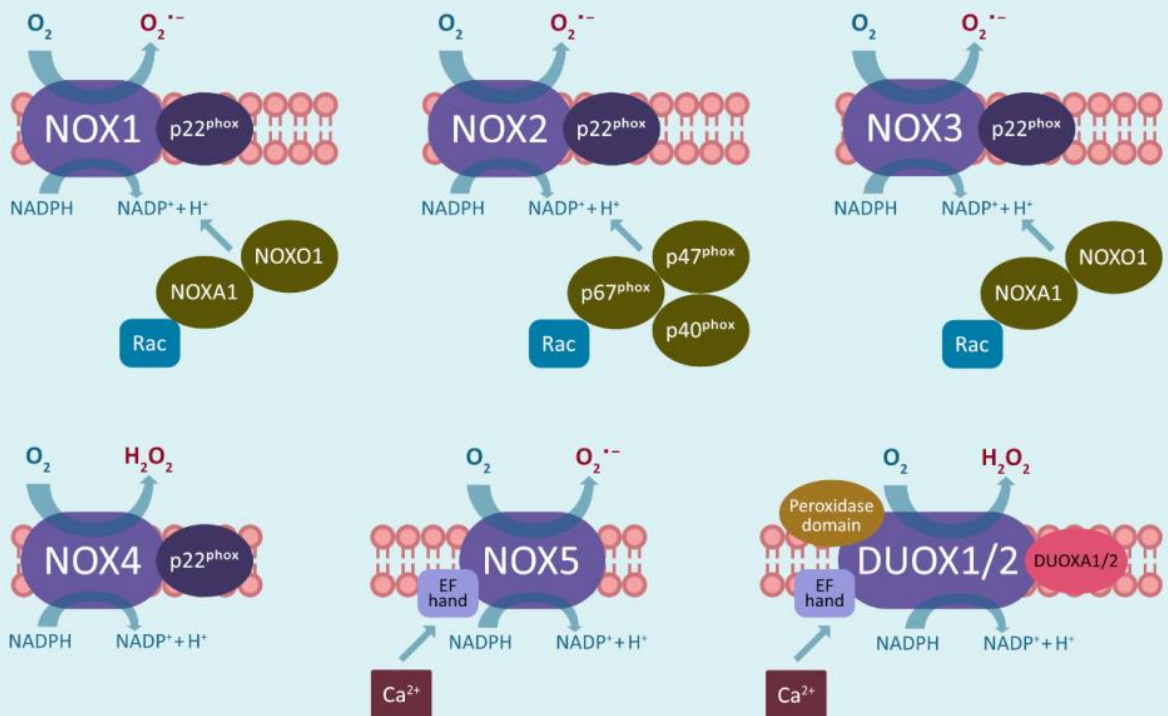

Figure 2.2: Structural overview of NOX family enzymes. NOX enzymes consist of 6 transmembrane domains with a NADPH-binding cytoplasmic C-terminal. NOX1, NOX2, NOX3 and NOX4 share the same structure and require association with p22 $2^{\text {phox }}$ as well as other cytosolic factors. NOX5 has an $\mathrm{N}$-terminal which contains EF hand $\mathrm{Ca}^{2+}$ binding sites. DUOX1 and DUOX2 share the same structure as NOX5 but also contain an extracellular peroxidase domain and require the cofactor DUOXA1/2. 


\subsection{Specific roles of NOX enzymes in IPF}

Recent studies indicate that expression or activation of several NOX enzymes is altered in the lungs of IPF patients, and may contribute to disease pathogenesis (2) (Figure 2.3).
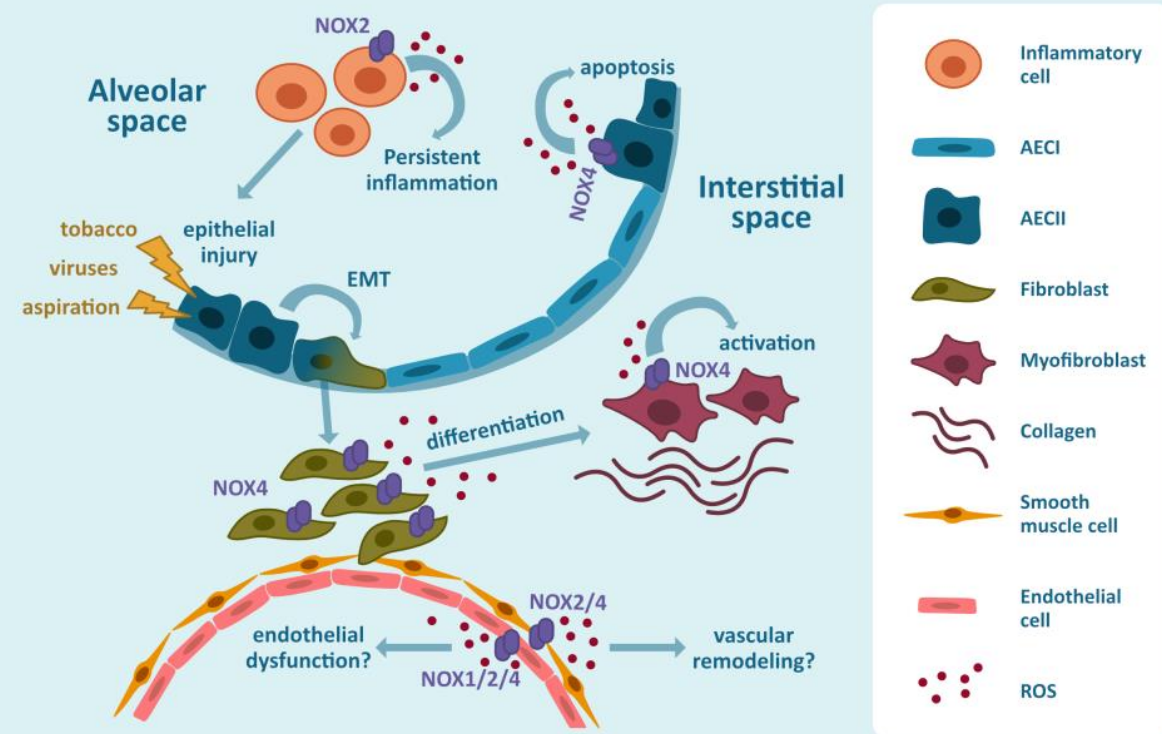

Figure 2.3: Proposed involvement of NOX enzymes in fibrotic responses. NOX-derived ROS facilitate pulmonary fibrosis by inducing apoptosis of AECII, EMT, proliferation and differentiation of fibroblasts, activation of myofibroblasts as well as proliferation of endothelial cells in response to injury to the lung epithelium.

Current evidence indicating alterations in NOX expression/activation in IPF, and their functional contribution to pulmonary fibrosis in animal models are summarized in Table 2.2 , and will be discussed in the following paragraphs. 
Table 2.2: Involvement of NOX enzymes in profibrotic processes in vitro, in vivo and in IPF patients.

\begin{tabular}{|c|c|c|c|c|c|}
\hline $\begin{array}{l}\text { NADPH } \\
\text { oxidase }\end{array}$ & $\begin{array}{l}\text { Cell/tissue } \\
\text { type }\end{array}$ & Model & $\begin{array}{l}\text { DUOX/NOX } \\
\text { activity }\end{array}$ & Key finding & Reference \\
\hline NOX1 & $\begin{array}{l}\text { Human } \\
\text { pulmonary } \\
\text { artery } \\
\text { endothelial } \\
\text { cells } \\
\text { C57BL/6 mice }\end{array}$ & $\begin{array}{l}\text { Radiation } \\
\text { Radiation }\end{array}$ & $\begin{array}{l}\text { Increased } \\
\text { Increased }\end{array}$ & $\begin{array}{l}\text { NOX1 inhibition by } \\
\text { shRNA reduces } \\
\text { intracellular ROS } \\
\text { and reduced } \\
\text { phenotypic changes } \\
\text { NOX1 is associated } \\
\text { with profibrotic } \\
\text { gene expression }\end{array}$ & $\begin{array}{l}\text { (137) } \\
\\
(137)\end{array}$ \\
\hline NOX2 & $\begin{array}{l}\text { BALF mice } \\
\text { C57BL/6 } \\
\text { gp91phox -/- } \\
\text { mice }\end{array}$ & $\begin{array}{l}\text { BLM } \\
\text { Carbon } \\
\text { nanotubes }\end{array}$ & $\begin{array}{l}\text { Increased } \\
\text { Increased }\end{array}$ & $\begin{array}{l}\text { NOX2-deficient mice } \\
\text { show a moderate } \\
\text { protection from } \\
\text { bleomycin-induced } \\
\text { lung fibrosis } \\
\text { NOX2 deficiency is } \\
\text { associated with the } \\
\text { suppression of the } \\
\text { pro-fibrotic } \\
\text { response, with } \\
\text { decreased TGF- } \beta \\
\text { and lower levels of } \\
\text { collagen deposition }\end{array}$ & $\begin{array}{l}(142) \\
(143)\end{array}$ \\
\hline NOX4 & $\begin{array}{l}\text { Human lung } \\
\text { fibroblasts } \\
\text { Human } \\
\text { alveolar } \\
\text { epithelial cells } \\
\text { Mouse } \\
\text { fibroblasts } \\
\text { Human } \\
\text { pulmonary } \\
\text { smooth } \\
\text { muscle cells }\end{array}$ & $\begin{array}{l}\text { TGF } \\
\text { BLM } \\
\text { BLM } \\
\text { IPF } \\
\text { patients }\end{array}$ & $\begin{array}{l}\text { Increased } \\
\text { Increased } \\
\text { Increased } \\
\text { Increased }\end{array}$ & $\begin{array}{l}\text { NOX4 regulates } \\
\text { myofibroblast } \\
\text { differentiation } \\
\text { NOX4 is a key player } \\
\text { in epithelial cell } \\
\text { death } \\
\text { NOX4 mediates } \\
\text { senescence and } \\
\text { apoptosis resistance } \\
\text { NOX4 is expressed } \\
\text { in thickened } \\
\text { pulmonary arteries }\end{array}$ & $\begin{array}{l}(26) \\
(146) \\
(20) \\
(154)\end{array}$ \\
\hline
\end{tabular}




\begin{tabular}{|l|l|l|l|l|l|}
\hline $\begin{array}{l}\text { Mouse } \\
\text { fibroblasts } \\
\text { and human } \\
\text { fibroblasts }\end{array}$ & BLM & Increased & $\begin{array}{l}\text { NOX4 is increased in } \\
\text { senescent } \\
\text { fibroblasts and } \\
\text { patients } \\
\text { contributes to } \\
\text { apoptosis resistance }\end{array}$ & (20) \\
$\begin{array}{l}\text { Human lung } \\
\text { fibroblasts }\end{array}$ & $\begin{array}{l}\text { IPF } \\
\text { patients }\end{array}$ & Increased & $\begin{array}{l}\text { NOX4 mediates } \\
\text { differentiation into } \\
\text { myofibroblasts }\end{array}$ & (147) \\
\hline
\end{tabular}

$\underline{N O X 1}$

NOX1 is expressed in epithelial and endothelial cells and in smooth muscle cells, with various described functions, but the specific contribution of NOX1 to IPF pathology is still largely unclear. Human pulmonary artery endothelial cells (HPAEC) transfected with NOX1-targeted shRNA show decreased levels of intracellular ROS as well as reduced fibrotic markers such as $\alpha$-SMA, vimentin and CD31, and NOX inhibition using VAS2870 was found to reduce collagen deposition in mouse lungs during radiation-induced fibrosis (137), even though the latter doesn't necessarily implicate NOX1 since VAS2870 is a nonselective NOX inhibitor. NOX1-mediated ROS by endothelial and epithelial cells have also been implicated in the induction of cell death in response to acute lung injury (138).

$\underline{N O X 2}$

NOX2 has been studied primarily in the context of phagocytic cells as a critical component of the innate immune response (139). Although the exact role of inflammation in the development in IPF is not completely clear, studies in patients with IPF have shown that ROS production from alveolar macrophages and neutrophils contributes to AEC death (87, 140) which could be regulated through NOX2 activity. Neutrophils isolated from BALF of IPF patients have higher $\mathrm{p} 47^{\text {phox }}$ and $\mathrm{p} 67^{\text {phox }}$ expression compared to healthy controls suggesting a specific role for NOX2 in alveolar neutrophils (141). Studies have shown that NOX2-deficient mice are protected from bleomycin (142) or carbon nanotube (143) induced pulmonary fibrosis but this may also involve non-immune cells that express NOX2. However, translation to IPF patients is rather difficult since initiation of fibrosis is driven through inflammation in these animal models which differs from the initiation process in patients where inflammation is seen as a secondary event instead (40). Conversely, it has been shown that NOX2 is important in the resolution of inflammation (144). NOX2 is also expressed in endothelial cells promoting endothelial proliferation (145); however, the specific role of NOX2 in the development of IPF still has to be elucidated.

$\underline{\text { NOX4 }}$

Among the seven members of the NOX family, NOX4 has been most commonly implicated in a variety of fibrotic diseases, including the liver, skin, kidney, heart, and lung. NOX4 is 58 
the only isoform that is highly upregulated in the lungs of IPF patients, mainly within epithelial cells (146) and (myo)fibroblasts (147), and is involved in several profibrotic processes.

NOX4 has been shown to contribute to cell death in hyperplastic type II AECs (146), and NOX4-deficient mice demonstrate significant less bleomycin-induced pulmonary fibrosis and associated AEC death (146), indicating that NOX4 contributes at least partially by inducing epithelial cell death $(26,146)$. As discussed above, EMT has been implicated as a feature of IPF, and the process of EMT may involve ROS-mediated mechanisms $(47,148$ 150). Studies in cancer cells indicate that EMT is largely driven by NOX4 $(151,152)$. NOX4 is highly expressed in pulmonary fibroblasts isolated from patients with IPF, and mediates fibroblast differentiation into myofibroblasts (147) and is also involved in the TGF- $\beta 1$ induced activation of myofibroblasts and collagen deposition (26). IPF lung fibroblasts display a senescent phenotype, and recent studies indicate that bleomycin-induced pulmonary fibrosis in aged mice (18 months) is characterized by the accumulation of senescent myofibroblasts, which involves a role for NOX4-induced ROS (20). Furthermore, NOX4 expression can also be regulated through histone modifications (153), which may be linked to the upregulation of NOX4 in senescent fibroblasts. Patients with IPF often acquire pulmonary hypertension, which could also be mediated by increased activity of NOX4, and NOX4 is expressed in thickened arteries of IPF patients (154). Indeed, smooth muscle cells (SMC) which are important in the regulation of pulmonary perfusion are activated by TGF- $\beta 1$ to induce NOX4 via a SMAD2/3-dependent pathway, leading to increased SMC proliferation (155). Endothelial cells lining pulmonary vessels also express more NOX4 at sites of angiogenesis within fibrotic regions and adjacent to fibrotic foci (156). TGF- $\beta$ has been implicated in endothelial cell death and linked to generation of NOX4-dependent ROS, although overexpression NOX4 was protective against TGF-induced endothelial cell apoptosis (157), indicating that the precise role for NOX4 in endothelial cells still remains unclear. In spite of the widespread evidence for a role of NOX4 in IPF pathology, with specific actions in different cell types, the precise downstream mechanisms by which NOX4-derived $\mathrm{H}_{2} \mathrm{O}_{2}$ mediates these responses are not known.

\section{Other NOXes}

Almost nothing is known with respect to other NOX enzymes in IPF. Interestingly, the process of EMT might also involve DUOX2, based on studies in human colon cancer cells (158). Conversely, our group recently observed that a loss of lung epithelial DUOX1, commonly observed in various cancers, can promote EMT (159). However, the relevance for DUOX in IPF or other fibrotic diseases has to date not been addressed. 


\section{Mitochondrial dysfunction as driver in IPF}

Mitochondrial dysfunction is a hallmark of age-related lung diseases (6) and has been associated with chronic lung diseases including IPF (160). Mitochondrial dysfunction is characterized by a loss of efficiency in the ETC resulting in an increased ROS generation in addition to a reduced membrane potential and altered mitochondrial function (161). IPF is also associated with changes in mitochondrial homeostasis, which is important for the maintenance of the redox-balance, mitochondrial DNA protection as well as AEC apoptosis and senescence, making cells more vulnerable to cellular stress (25).

\subsection{Mitochondria in cell homeostasis}

Mitochondria are double membrane-containing organelles that are typically between 0.75 and $3 \mu \mathrm{m}$ in diameter, although they are approximately three times larger in type II AECs compared to other lung cells such as type I AECs $(162,163)$. Their main function is to produce cellular energy in the form of ATP through oxidative phosphorylation (OXPHOS), thereby generating more than $90 \%$ of the required metabolic energy in most cells (164). In addition, mitochondria are also important other cell signaling processes involved in e.g. differentiation or apoptosis, and appropriate mitochondrial function is tightly controlled by mitochondrial biogenesis, fission/fusion dynamics and mitophagy (25). In response to cellular stress or injury, mitochondria can rapidly adapt their behavior by changing mitochondrial fusion (merging) and fission (division) dynamics, which will alter the ATP production in line with cellular demand (25).

During differentiation from type II to type I AECs, type II AECs reduce the number and size of mitochondria to adapt to cellular stress, which results in lower energy demanding type I AECs (162). Increased fusion promotes the formation of elongated mitochondria and is a stress-resolving mechanism in which the fused mitochondria are protected from degradation to respond to the cells higher energy demand to repair cellular damage (165). Conversely, increased fission or reduced fusion increases mitochondrial fragmentation, which under severe stress promotes mitochondrial autophagy, also called mitophagy, a process in which damaged mitochondria are removed to maintain mitochondrial homeostasis (166). Similarly, mitophagy also regulates mtROS production indirectly by removing dysfunctional mitochondria with high mtROS production (167). The intrinsic mitochondria-regulated cell apoptosis pathway is activated by various fibrotic stimuli, including hypoxia, oxidative stress and DNA damage, that in turn stimulate the proapoptotic $\mathrm{Bcl}-2$ family members in the mitochondria increasing mitochondrial membrane permeability which causes the release of cytotoxic proteins into the cytosol such as cytochrome $C$ thereby activating caspase 3 and $9(168,169)$. These regulatory mechanisms are important for mitochondrial homeostasis, and if dysregulated mitochondrial dysfunction can occur predisposing to disease. 


\subsection{Mitochondrial dysfunction in IPF}

Mitochondrial dysregulation of mitochondrial dynamics disrupts adaption to cellular stress, making lung cells more vulnerable to (oxidative) injury thereby promoting pulmonary fibrosis. Recently, it has been identified that epithelial cells as well as fibroblasts of IPF patients show signatures of dysfunctional mitochondria and disturbed mitochondrial dynamics $(12,25)$.

\section{Dysregulated fission and fusion dynamics}

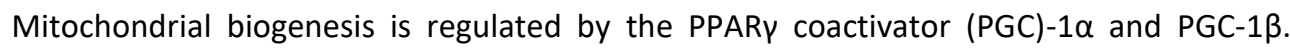
PGC-1 is capable of activating nuclear respiratory factors (NRF-1 and NRF-2), which are essential for mitochondrial biogenesis (170). Of note, these nuclear respiratory factors are distinct from nuclear factor erythroid 2-related factors, which are commonly denoted as Nrf1 and Nrf2, a confusion made even worse by observations that PGC-1 $\alpha$ can also upregulate Nrf2 (171). Additionally, mitochondrial biogenesis requires the replication and synthesis of mitochondrial DNA which is regulated by the mitochondrial transcription factor A (TFAM) (172). With aging, the capacity for mitochondrial biogenesis declines through reduction in upstream activators of PGC-1 (173), thereby slowing down mitochondrial turnover. Additionally, expression of PGC-1 $\alpha$ is reduced in IPF patients and in murine models of IPF $(25,174)$. AEC type II isolated from aged mice show an increase of mitochondrial fusion markers but a decrease in mitochondrial fission (24) resulting in an increased mitochondrial area. Increased fusion can suggest a need for more energy under stress conditions thereby promoting cell survival (175).

\section{Alterations in mitophagy}

Mitophagy is essential to maintain normal cellular function by regulating the number of mitochondria and to prevent apoptosis through the removal of dysfunctional mitochondria by autophagy (176). Deficiency in mitophagy has been associated with the development of pulmonary fibrosis in response to injury (177). Selective mitophagy of damaged mitochondria occurs through the PTEN-induced putative kinase 1 (PINK1) that accumulates on defective mitochondria acting as a marker for mitochondrial damage (178). Subsequently, PINK1 activates the E3-ubiquitin ligase Parkin in the cytosol by phosphorylation, which labels the outer membrane of dysfunctional mitochondria for trafficking to the autophagosome (24). With aging and also in lungs of IPF patients, a reduction in autophagic activity, indicated by a reduced number of autophagosomes and expression of LC3 and p62, has been described (179). This decrease in mitophagy has been associated with a decrease in PINK1 expression in IPF lungs, thereby promoting the accumulation of damaged mitochondria in IPF (24). However, short-term stimulation with TGF- $\beta 1$ induces PINK1 expression in BEAS-2B cells thereby protecting epithelial cells by removal of damaged mitochondria and evading apoptosis whereas long-term TGF- $\beta 1$ stimulation (24 hrs) reduces PINK1 in lung fibroblasts (177). Interestingly, type II AECS 
from lungs of IPF patients express less PINK1 suggesting that PINK1 deficiency impairs mitochondrial function of AECs (24). Furthermore, PINK1 deficiency results in the accumulation of dysfunctional mitochondria and exacerbates bleomycin-induced pulmonary fibrosis which is also associated with increased apoptosis (180). In fibroblasts, TGF- $\beta 1$ inhibits autophagy leading to an increase in profibrotic gene expression such as $\alpha$ smooth muscle actin and fibronectin contributing to myofibroblast differentiation (179). Taken together, short-term exposure of TGF- $\beta 1$ leads to the initial stabilization of PINK1 thereby inducing mitophagy, whereas long-term exposure results in impaired mitophagy through downregulation of various proteins involved in PINK1 activation promoting AEC apoptosis.

\section{Mitochondria-mediated AEC death}

Mitochondrial dysfunction is suggested to represent a key mechanism for epithelial cell apoptosis in pulmonary fibrosis $(160,181)$ as type II AECs are more susceptible to apoptosis when mitochondrial function is impaired (24). Additionally, mitochondrial DNA (mtDNA) damage is linked to oxidative injury and cellular stress driving apoptotic and senescence pathways and thereby contributing to pulmonary fibrosis (38). As mtDNA is more susceptible to oxidative damage compared to nuclear DNA due to its lack of histones and its proximity to the ETC (163), mtDNA repair is essential for mitochondrial dynamics. Experimental models of pulmonary fibrosis contain a higher amount of damaged mtDNA $(182,183)$ which is linked to insufficient mtDNA repair (25). Eight-oxoguanine DNA glycosylase 1 (8-OGG1) is an important base excision repair enzyme in mtDNA repair and deficiency promotes pulmonary fibrosis (184). Knockout of OGG1 has been shown to augment (185) whereas overexpression of mitochondria-targeted OGG1 prevents oxidantinduced AEC apoptosis (186) in asbestos-induced models of pulmonary fibrosis.

Mitochondrial damage associated molecular patterns (mtDAMPs) have also been linked to mitochondria-mediated apoptosis (187). MtDAMPs are mitochondrial-derived molecules which are important for proper cell signaling but can also behave as damage signal in response to tissue injury, thereby activating pathogen recognition receptors (PRR) (162). One of the most important mtDAMPs is the release of oxidized and /or fragmented mtDNA from damaged mitochondria which can trigger apoptosis (162) and inflammation (188). Indeed, mtDNA is increased in the lungs and blood of patients with IPF (189). Additionally, it has been shown that mtDNA release can trigger TGF- $\beta 1$ release from AECs in paraquat-induced pulmonary fibrosis (190) and inflammasome activation in macrophages (188). Excessive ATP release by damaged and apoptotic AECs can also act as mtDAMP, activating the purinergic receptor P2X7 (162), and is increased in bleomycininduced pulmonary fibrosis (191). 


\section{Dysregulated mtROS production}

During OXPHOS, $\mathrm{O}_{2}$ is reduced to $\mathrm{H}_{2} \mathrm{O}$ which leads to the formation of $\mathrm{O}_{2}{ }^{--}$and/or $\mathrm{H}_{2} \mathrm{O}_{2}$ due to electron leakage in the mitochondrial ETC and these mitochondrial ROS (mtROS) are thought to promote pulmonary fibrosis (192). The mitochondrial ETC comprises five large protein complexes: complex 1 (NADH-coenzyme Q oxidoreductase), complex II (succinate/coenzyme Q oxidoreductase), complex III (Q-cytochrome c oxidoreductase), complex IV (cytochrome c oxidase) and complex V (ATP synthase) (193). MtROS are generated at eleven distinct mitochondrial sites within complex I, II and III (194) and are the main sites of electron leakage to oxygen to produce $\mathrm{O}_{2}{ }^{-}$and $\mathrm{H}_{2} \mathrm{O}_{2}$ (195). Complex III and mitochondrial glycerol 3-phosphate dehydrogenase generate $\mathrm{O}_{2}{ }^{\circ-}$ on the cytosolic side of the mitochondrial inner membrane as well as the matrix whereas the other sites generate $\mathrm{O}_{2}{ }^{\circ-}$ and/or $\mathrm{H}_{2} \mathrm{O}_{2}$ exclusively in the matrix (195). Effective regulation of $\mathrm{mtROS}$ as well as the capacity to scavenge them by antioxidants declines with age making the lungs more susceptible towards oxidative damage (196). Normally, mtROS production is tightly regulated, however when dysregulated they promote mitochondrial dysfunction, apoptosis and cellular (mitochondrial) DNA damage (25).

TGF- $\beta$ stimulation of fibroblasts from IPF patients results in increased mtROS compared to similar stimulation of fibroblasts from control subjects (192). Similarly, mtROS generation is also increased in bleomycin-induced pulmonary fibrosis (182). The profibrotic cytokine TGF- $\beta 1$ enhances mtROS through the inhibition of mitochondrial complex IV in lung epithelial cells (197). Genetic disruption of mitochondrial complex III-generated ROS attenuates TGF- $\beta 1$-induced profibrotic gene expression during myofibroblast differentiation (192), further indicating the important role of mtROS in the development of pulmonary fibrosis. Moreover, studies have suggested a key role for TGF-induced mtROS production in the induction of senescence in epithelial cells (197). Dysregulated mtROS generation is not only a key player in profibrotic signaling but also plays a role in mitochondrial DNA damage leading to apoptosis. Persistent mitochondrial DNA damage can result in the activation of apoptosis (198). Furthermore, oxidative damage to mitochondrial DNA can lead to mutations resulting in the synthesis of defective ETC components, thereby further enhancing the production of mtROS (184). In agreement with these observations, mice that overexpress mitochondrial catalase show lower level of mtROS-induced mitochondrial DNA damage than their wildtype littermates and are protected from bleomycin-induced pulmonary fibrosis (182). Interestingly, it has been shown that mtROS can also damage nuclear DNA which in turn causes mitochondrial dysfunction by the activation of nucleus-to-mitochondria-signaling pathways (199). However, more in vivo studies are needed to elucidate the exact role for mtROS in the development of IPF. In addition to interference with normal cellular function, mitochondrial dysfunction has also been implicated as a driver for EMT in cancer cells (200). 
In summary, redox alterations induced by mitochondrial dysfunction contribute to IPF pathophysiology, which may also be associated with their interactions with NOX activation $(192,201)$, as will be discussed in the next section. 


\section{Cross-talk between NOX enzymes and mitochondria}

Since most cell types express multiple NOX isoforms and all mammalian cells contain various amounts of mitochondria, it is often difficult to determine the precise cellular source of ROS, and this may in fact concerted input from various sources. Indeed, over the past several years, various lines of evidence support the existence of cross-talk mechanisms between different NOX isoforms, and also between NOX enzymes and mitochondria, thereby potentially altering mitochondrial function or NOX activity (202). Such interactions between NOX and mitochondria are also reciprocal, i.e. activation of NOX can increase mtROS production but mitochondria (via mtROS) can also contribute to activation of NOX. The significance of such NOX-mitochondria interactions and their potential relevance for IPF will be discussed in the following sections.

\subsection{Stimulation of mtROS production by NOX enzymes}

The first studies indicating a role for NOXes in mitochondrial dysfunction have been in the context angiotensin II (AngII) signaling in vascular biology (203). Angll is an important hormone in the renin-angiotensin-system and induces vascular dysfunction in part by activation of vascular NOX1 and NOX2. AngIl also leads to increased vascular endothelial mtROS production, which was decreased by deletion of the NOX cofactor $\mathrm{p} 22^{\text {phox }}$ or by preincubation with the NOX inhibitor apocynin (204, 205). Additionally, Angll-induced NOX activation was associated with opening of redox-sensitive mitoK ATP channels, leading to depolarization of mitochondrial membrane potential and subsequent mitochondrial ROS generation $(204,206)$. More recent studies of VEGF-induced angiogenesis indicated a sequential mechanism involving successive activation NOX2, NOX4, and mtROS production (205), and VEGF-induced endothelial cell migration and proliferation could be inhibited by silencing either NOX2 or NOX4 or by mitochondrial targeting of catalase (205). Crosstalk between mitochondria and NOX has also been implicated in the development of nitrate tolerance and associated endothelial dysfunction $(207,208)$, which could be prevented by selective inhibition of $\mathrm{mtROS}$ production using rotenone or the mitochondrial pore blocker cyclosporine A, or by inhibition of NOX activity by chimeric peptide that interferes with assembly of p47 phox and NOX2 (209). Taken together, these studies indicate that NOX activity increases mtROS production, although the exact mechanisms still remain to be elucidated.

\subsection{Activation of NOX enzymes by mtROS}

ROS production by pulmonary artery smooth muscle during acute hypoxia was found to originate from mitochondria, and subsequent NOX activation, based on inhibition of increase in NOX activity, measured by $\mathrm{O}_{2}{ }^{-*}$-dependent cytochrome $\mathrm{C}$ reduction, by the complex I inhibitor rotenone (210). A study using human leukocytes indicated that NOX2 activation depends on mtROS formation, as it was enhanced by deletion of mitochondrial 
SOD2 (211). Similarly, Angll-induced endothelial dysfunction, which involves NOX2, was more pronounced in SOD2 knockout mice (211). NOX activation by Angll-induced mtROS production was also demonstrated by inhibitory effects of mitochondrial-targeted antioxidant mitoTEMPO (212). Studies with the human embryonic kidney cell line 293T indicated that mtROS can also activate NOX1, under conditions of serum starvation, by promoting phosphoinositide 3-kinase (PI3K) and Rac1 activation, events that were inhibited by rotenone (213). Separate studies indicate that mitochondrial dysfunction (perhaps through mtROS) can also regulate NOX1 expression (203). Hence, emerging lines of evidence indicate a contribution of mtROS in activation of various NOX enzymes, including NOX1 and NOX2. More relevant to IPF, mitochondria also display cross-talk with NOX4, as will be discussed in the next section.

\subsection{Crosstalk between NOX4 and mitochondria - potential role in IPF?}

As mentioned earlier, NOX4 contributes to critical features of pulmonary fibrosis. Various lines of evidence indicate that NOX4 can be localized to mitochondria since NOX4 contains a 73-amino-acid long mitochondrial targeting signal in its $\mathrm{N}$-terminus allowing it to localize to the inner mitochondrial membrane $(136,214)$. As such, NOX4 has been reported to be expressed in the mitochondria of rat kidney cortex (215), in cardiac myocytes (216), and in cancer cells (214), and it has been suggested that mitochondrial NOX4-produced ROS are implicated in several disease pathologies through modulation of senescence, apoptosis and carcinogenesis. Indeed, NOX4-induced mtROS production in cardiac myocytes has been implicated in cell apoptosis and cardiac hypertrophy (217). Furthermore, cardiac NOX4 contributes to cardiac failure through the generation of ROS (216), and NOX4deficient mice have attenuated mtROS generation and mitochondrial dysfunction indicating interplay between NOX4 and mtROS in the failing heart (216). It is also worth noting that inhibition of NOX4 may also lead to a more basal reduced state (based on $N A D(P)+/ N A D(P) H$ ratio) and can paradoxically enhance mtROS production under conditions of e.g. ischemia in the heart (218).

Mitochondrial activity is regulated by various extracellular and intracellular signaling pathways, including SRC kinases (219), which can be subject to NOX-dependent redox modulation (220). Conversely, the SRC family kinase member FYN was identified as a negative regulator of NOX4, by promoting NOX4 phosphorylation on tyrosine 566 (217). Studies in human endothelial cells indicate that NOX4 can also directly interact with mtROS production through inhibition of mitochondrial complex $\mathrm{I}$, thereby reducing the mitochondrial respiratory capacity and contributing to mitochondrial dysfunction (221), although the specific subcellular localization of NOX4 was not determined in this study. In fact, NOX4-depleted endothelial cells showed a reduction in $\mathrm{H}_{2} \mathrm{O}_{2}$ production in the mitochondria as well as in the cytosol. Another study identified mitochondrial NOX4dependent ROS production as a key mediator in apoptosis of kidney tubular cells in 
response to ANGII (222). Most examples of connections between NOX4 and mitochondria stem from cardiovascular studies, but recent studies also highlight the presence of NOX4mitochondria crosstalk in IPF, and suggest a role for NOX4 in mitochondrial dysfunction, associated with altered biogenesis, increased glycolysis and increased ATP degradation $(25,223)$.

It was recently reported that NOX4 is able to repress mitochondrial biogenesis in lung fibroblasts through direct inhibition of Nrf2 and TFAM independent of PGC-1 $\alpha$ (28). Indeed, inhibition of NOX4 by genetic or pharmaceutical approaches resulted in increased mitochondrial biogenesis, illustrated by increased mitochondrial-to-nuclear DNA ratio (28). Unfortunately, these studies only evaluated effects of NOX4 deletion and not of NOX4 overexpression, as it would be observed in IPF. NOX4 has been shown to influence mitochondrial biogenesis through its interaction with PDIP38, also known as DNA polymerase delta interacting protein 2 (POLDIP2), which is involved in DNA replication but is also localized to mitochondria where it associates with mitochondrial nucleotides (224, 225). This interaction could indicate that NOX4 is involved in mtDNA replication and repair via its interaction with PDIP38 or via the production of ROS thereby modifying proteins involved in mtDNA maintenance. NOX4 can contribute to the increase of mtROS via redox signaling, thereby modulating mitochondrial functions including the activation of PKC, mitoKATP and TRX2 (206). Intriguingly, recent studies also implicated mitochondrial NOX4 as an energetic sensor in cancer cells (226). During normal respiration, OXPHOS-driven ATP production binds NOX4 through an ATP-binding motif, thereby minimizing constitutive NOX4 activity. During transition of OXPHOS to aerobic glycolysis, as it is observed in cancer, mitochondrial ATP production is reduced and results in increased NOX4 activity (226). Metabolic alterations in IPF indicated increased ATP degradation (223) which would similarly result in enhanced NOX4 activity. Interestingly, it has been suggested that inhibition of aerobic glycolysis might be a beneficial target in IPF as well $(227,228)$. Administration of the mitochondria-targeted antioxidant mitoQ was found to attenuate NOX4 expression in fibroblasts from IPF patients (192), further highlighting the important role of mitochondria-produced ROS in NOX4 activation.

In summary, the various forms of crosstalk between NOX4 and mitochondria could have important consequences for IPF manifestation/progression (Figure 4). Increased NOX4 activity in IPF promotes AEC cell death as well as (myo)fibroblast activation causing excessive collagen deposition $(146,147)$, and could be mediated by NOX4-dependent mtROS production. Mitochondrial ROS promotes mitochondrial DNA damage and could also directly contribute to NOX4 activation, thereby promoting a fibrotic vicious circle (192). Moreover, negative effects of NOX4 on mitochondrial biogenesis could further drive mitochondrial dysfunction. While these NOX4-mitochondria interactions may be related to localization of NOX4 in mitochondria, evidence for such localization rest primarily on 
detection with antibodies with unknown specificity, and it typically not associated with costainings of $\mathrm{p} 22^{\text {phox }}$, which is important for its activity. Hence, the exact role of NOX4 in mitochondria still remains unclear, and further research is needed to more firmly establish NOX4 as a mitochondrial protein, and its reciprocal interactions with mitochondrial processes.

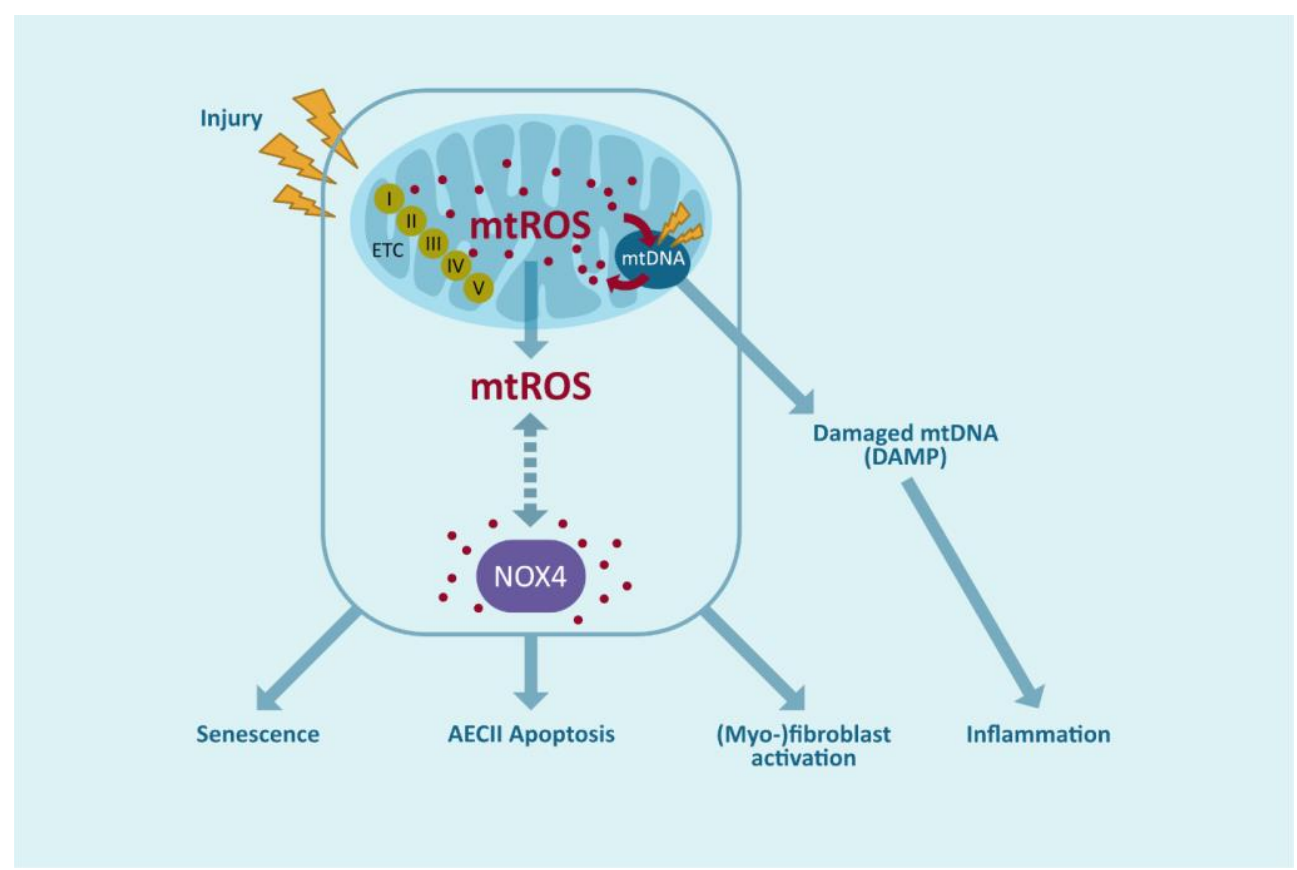

Figure 2.4: Interplay between mitochondria and NOX4. Release of ROS by mitochondria can trigger ROS production by NOX4 outside the mitochondria and possibly also within the mitochondria. Mitochondrial ROS leads to mtDNA damage which can contribute to mitochondrial dysfunction leading to AEC death. Additionally, mtDNA damage further increases mtROS. Oxidized mtDNA can also act as a mtDAMP outside the mitochondria and inducing inflammatory processes. These signaling cascades may further amplify NOX4-mediated effects including fibroblast differentiation, myofibroblast activation and AEC death. 


\section{Redox-modulatory therapeutic strategies}

Based on the various lines of evidence implicating a redox imbalance in IPF, several redoxbased therapeutic strategies have been proposed focusing at quenching ROS or restoring the disturbed redox-balance. For example, several studies have addressed the ability of $\mathrm{N}$ acetyl-cysteine (NAC), a precursor of GSH, to mitigate IPF. However, in spite of demonstrated encouraging results in various in vitro studies (229) as well as experimental fibrosis models $(230,231)$, NAC supplementation has failed to be fully effective in the clinic (232-234) because of variable effects in patients with IPF. These variable effects may be due to differences in dose of drug administration, delivery method as well as the patient population. Several studies have suggested that inhaled NAC (352.4 mg, two times daily) improves lung function in early stage IPF $(96,235,236)$, indicating that local antioxidant therapy might be more beneficial in an early stage of IPF to counteract ROSinduced damage and the underlying inflammation. Oral administration of NAC (600 mg, 3 times daily) on the other hand had variable outcomes. In the PANTHER trial no significant improvement of lung function could be observed in mild-to-moderate IPF compared to the placebo (237) whereas it was been reported before in the IFIGENIA trial that the same dose of NAC in combination with prednisone and azathioprine reduced decline of lung function (238). In advanced IPF, NAC monotherapy showed no beneficial effect, however, combination therapy has been suggested to improve lung function (239). Consequently, it is important to investigate specific subgroups in IPF who could benefit from NAC as monotherapy or in combination therapy that has been suggested to be more beneficial in an advanced stage.

Other studies have explored the ability of antioxidant food supplements, such as quercetin and resveratrol, to reduce oxidative stress in profibrotic responses in vitro (95, 240-242) and in animal models of pulmonary fibrosis (243-246). While these compounds have reported antioxidant activity in vitro, they might act by alternative mechanisms and are also capable of activating Nrf2 (247). Intriguingly, a recent study described that quercetin reverses bleomycin-induced pulmonary fibrosis in aged mice through the reduction of various senescence markers and SASP (248). Furthermore, transcriptome analysis of pathways involved in senescence and drugs that interfere with these pathways yielded dasatinib (a SRC/ABL protein kinase inhibitor) and quercetin (a putative antioxidant but also an inhibitor of various kinases) as potent senolytic drugs (249). Indeed, the combination of dasatinib with quercetin $(D+Q)$ was found to kill senescent fibroblasts in a mouse model of bleomycin-induced pulmonary fibrosis and thereby improve lung function (38). While these findings do not directly indicate antioxidant-based mechanisms, such a mechanism could contribute to the observed inhibitory effects, especially in combination with other antifibrotic drugs. The combination of $D+Q$ is 
currently investigated in a clinical trial (NCT02874989) to determine the effects on proinflammatory cells in IPF.

In addition to approaches using general scavengers of ROS, alternative strategies have focused on specifically modulating the activity of redox systems. For example, based on observed loss of GRX1 activity in IPF, and corresponding increases in S-glutathionylation of Fas and caspase activation, administration of recombinant GRX1 has been explored as a therapeutic strategy, and preclinical studies in mice indicate the ability of GRX1 to inhibit and even promote reversal of pulmonary fibrosis in experimental models in mice (110).

Based on the diverse and specific actions of ROS produced by specific NOX enzymes or by mitochondria, as discussed in this review, it would seem more fruitful to specifically target (enzymatic) sources of ROS that are dysregulated in IPF, rather than more generic approaches to neutralize ROS or affect redox systems, as these latter could also interfere with normal physiological functions of ROS, and thus exert unwanted effects $(250,251)$. Indeed, recent approaches have focused primarily on the specific roles of NOX enzymes and mitochondria in ROS production (82). Pharmacological approaches to inhibit NOX4 are being developed as potential therapeutic strategies in IPF treatment. Indeed, a smallmolecular inhibitor that selectively targets NOX4 as well as NOX1 (GKT137831) was found to minimize bleomycin-induced fibrosis in a mouse model, by reducing fibroblast activation and collagen deposition as well as epithelial cell death $(2,156)$. Moreover, GKT137831 treatment in IPF lung fibroblasts results in reduced markers of senescence and increased the susceptibility to apoptosis suggesting that NOX4 contributes to senescence (20) but further studies with specific inhibitors are needed to establish this hypothesis.

Based on the accumulating evidence that associates mtROS with IPF development, various mitochondria-targeted therapies have been developed to improve mitochondrial biogenesis, activate mitophagy, inhibit mtROS regulated apoptosis and scavenge mtROS in general. The mitochondrial antioxidant mitoQ, a ubiquinone conjugated to a lipophilic triphenylphosphonium cation that accumulates within mitochondria and is reduced to the antioxidant ubiquinol, has been shown to attenuate liver fibrosis (252) and reduce TGFinduced profibrotic gene expression in human fibroblasts (192). Another mitochondrialtargeted small molecule, XBJ-5-131, was shown to improve the survival of neurons in a mouse model of Huntington disease through inhibition of oxidative (mitochondrial) DNA damage $(253,254)$. Another example is the antidiabetic drug, metformin, which inhibits the activity of complex I, thereby reducing mtROS. Metformin has been suggested as antifibrotic drug as it interferes with TGF- $\beta 1$ signaling, inhibits myofibroblast differentiation (255), and reduces collagen deposition in BLM-induced pulmonary fibrosis (256). A recent review, however, indicated no beneficial effect on lung function in patients with IPF (257). Overall, these various findings suggest that selective targeting of specific NOX isoforms or mitochondrial ROS production may be beneficial in management of IPF, especially in light 
of studies indicating that redox alterations induced by mitochondrial dysfunction may also be associated with their interactions with NOX activation $(192,201)$. 


\section{Conclusion and future directions}

Although IPF is well-recognized for its devastating nature and poor prognosis, only minimal advances have been made in the last years to effectively treat patients with IPF. Recent FDA-approved drugs pirfenidone and nintedanib have only elicited modest improvements in disease progression and cannot ameliorate the poor survival associated with IPF (258). Moreover, they do not work for all IPF patients, indicating the need to investigate more treatment strategies (259). As discussed in this review, IPF is characterized by a dysregulated redox homeostasis and mitochondrial dysfunction. Yet, attempts to reduce ROS with general antioxidants such as $\mathrm{N}$-acetyl cysteine have largely been unsuccessful in the clinic, which is likely due to our incomplete understanding of specific redox-based mechanisms that contribute to IPF development, and the fact that such general antioxidant strategies would also interfere with beneficial homeostatic functions of ROS. However, we would caution against negative conclusions based on unsuccessful studies and dismiss the potential importance of ROS-based mechanisms in IPF pathology, because of accumulating evidence implicating specific redox-based mechanisms (increased NOX activation, mitochondrial dysfunction) that ought to be targeted more directly. In this regard, it would be prudent to explore the efficacy of selective targeting of NOX4, based on compelling preclinical studies, or approaches to specifically inhibit mtROS such as MitoQ and MitoTEMPO, which have shown some success in experimental mouse models of pulmonary fibrosis. Another common challenge with such pharmaceutical approaches is successful targeting of fibroblast foci, which may be poorly accessible to systemically administered drugs.

Another reason for our relatively limited understanding of ROS in IPF is the fact that detection of specific ROS pathways in clinical specimens is exceedingly difficult (especially reversible modifications that may be more important in dysregulated cell signaling than stable protein oxidation products). In this regard, recent studies of protein $S$ glutathionylation in IPF in association with clinical outcomes (110) are particularly enlightening. Moreover, addressing the causality of specific ROS pathways would require appropriate animal models, and currently used models of pulmonary fibrosis do not adequately model the pathophysiology of human IPF and its progression. Also, while IPF is well-known to be age-associated, most animal studies are still using young animals and thus do not recapitulate the contribution of age-associated alterations on specific redoxbased pathways. Although costly, future studies should address pharmacological strategies in age-appropriate models. Another problem with studies of ROS in disease models such as IPF is that they typically address the importance of a specific NOX enzyme or antioxidant system throughout the disease model, ie. during the initiation and development of the disease, which may not necessarily translate into clinical practice if pre-existing fibrosis is not reversed. In this light, some recent studies suggesting that 
antioxidant-based approached can actually reverse fibrosis, at least in animal models (110, 256), are particularly encouraging.

Although evidence is growing for a role of specific ROS pathways in IPF (e.g. NOX4, mtROS), we still know relatively little with respect to the precise molecular mechanisms involved (ie. what are the critical cellular targets for e.g. NOX4-derived ROS that facilitate AEC apoptosis or fibroblast activation). In light of emerging evidence that NOX4 may be localized to mitochondria and that mitochondria and NOX4 exert reciprocal interactions, future studies should aim at unraveling the molecular redox mechanisms underlying such interactions, for example by using approaches to interfere with mitochondrial NOX4 signaling. Successful identification of specific cellular targets for NOX4-derived ROS or for mtROS in IPF may reveal alternative and potentially more attractive therapeutic targets for treatment of IPF. Given the rapid expansion of redox-based research in recent years, and development of novel research tools to assess site-specific redox events in subcellular compartments or in specific proteins, it is anticipated that our understanding of such NOX4-mitochondria interactions will soon increase and may indeed lead to novel redoxbased therapeutic strategies that may prove to be beneficial in improving the survival and the quality of life in patients suffering from IPF. 


\section{Acknowledgements}

$\mathrm{CV}$ is supported by the Nutrim Graduate Programme (Maastricht University). AvdV gratefully acknowledges research support from the National Heart Lung and Blood Institute (R01 grants HL085646 and HL138708) and the National Institute on Aging (R21 AG055325). Additionally, the authors would like to thank Birgit Hettler for her assistance in designing the figures. 


\section{References}

1. du Bois RM. Strategies for treating idiopathic pulmonary fibrosis. Nature reviews Drug discovery. 2010;9(2):129-40.

2. Hecker L, Cheng J, Thannickal VJ. Targeting NOX enzymes in pulmonary fibrosis. Cell Mol Life Sci. 2012;69(14):2365-71.

3. Pardo A, Selman M. Lung Fibroblasts, Aging, and Idiopathic Pulmonary Fibrosis. Annals of the American Thoracic Society. 2016;13(Supplement_5):S417-S21.

4. World health organization. Ageing and health. 2018. http://www.who.int/newsroom/fact-sheets/detail/ageing-and-health (accessed 26 Nov 2018).

5. Meiners S, Eickelberg O, Konigshoff M. Hallmarks of the ageing lung. Eur Respir J. 2015;45(3):807-27.

6. Lopez-Otin C, Blasco MA, Partridge L, Serrano M, Kroemer G. The hallmarks of aging. Cell. 2013;153(6):1194-217.

7. Yang IV, Schwartz DA. Epigenetics of idiopathic pulmonary fibrosis. Transl Res. 2015;165(1):48-60.

8. $\quad$ Alder JK, Chen JJ, Lancaster L, Danoff S, Su SC, Cogan JD, et al. Short telomeres are a risk factor for idiopathic pulmonary fibrosis. Proc Natl Acad Sci U S A. 2008;105(35):13051-6.

9. Armanios M. Telomerase and idiopathic pulmonary fibrosis. Mutat Res. 2012;730(1-2):52-

8.

10. Naikawadi RP, Disayabutr S, Mallavia B, Donne ML, Green G, La JL, et al. Telomere dysfunction in alveolar epithelial cells causes lung remodeling and fibrosis. JCl Insight. 2016;1(14):e86704.

11. Alvarez D, Cardenes N, Sellares J, Bueno M, Corey C, Hanumanthu VS, et al. IPF lung fibroblasts have a senescent phenotype. Am J Physiol Lung Cell Mol Physiol. 2017;313(6):L1164-L73. 12. Mora AL, Bueno M, Rojas M. Mitochondria in the spotlight of aging and idiopathic pulmonary fibrosis. J Clin Invest. 2017;127(2):405-14.

13. Minagawa S, Araya J, Numata T, Nojiri S, Hara H, Yumino Y, et al. Accelerated epithelial cell senescence in IPF and the inhibitory role of SIRT6 in TGF-beta-induced senescence of human bronchial epithelial cells. Am J Physiol Lung Cell Mol Physiol. 2011;300(3):L391-401.

14. Kinnula VL, Fattman CL, Tan RJ, Oury TD. Oxidative stress in pulmonary fibrosis: a possible role for redox modulatory therapy. Am J Respir Crit Care Med. 2005;172(4):417-22.

15. Montuschi P, Ciabattoni G, Paredi P, Pantelidis P, du Bois RM, Kharitonov SA, et al. 8Isoprostane as a biomarker of oxidative stress in interstitial lung diseases. American journal of respiratory and critical care medicine. 1998;158(5 Pt 1):1524-7.

16. Kurundkar A, Thannickal VJ. Redox mechanisms in age-related lung fibrosis. Redox biology. 2016;9:67-76.

17. Bargagli E, Olivieri C, Bennett D, Prasse A, Muller-Quernheim J, Rottoli P. Oxidative stress in the pathogenesis of diffuse lung diseases: a review. Respir Med. 2009;103(9):1245-56.

18. Psathakis K, Mermigkis D, Papatheodorou G, Loukides S, Panagou P, Polychronopoulos V, et al. Exhaled markers of oxidative stress in idiopathic pulmonary fibrosis. Eur J Clin Invest. 2006;36(5):362-7.

19. Lenz AG, Costabel U, Maier KL. Oxidized BAL fluid proteins in patients with interstitial lung diseases. The European respiratory journal. 1996;9(2):307-12.

20. Hecker L, Logsdon NJ, Kurundkar D, Kurundkar A, Bernard K, Hock T, et al. Reversal of persistent fibrosis in aging by targeting Nox4-Nrf2 redox imbalance. Sci Transl Med. 2014;6(231):231ra47.

21. Cheresh P, Kim SJ, Tulasiram S, Kamp DW. Oxidative stress and pulmonary fibrosis. Biochim Biophys Acta. 2013;1832(7):1028-40. 
22. Sena LA, Chandel NS. Physiological roles of mitochondrial reactive oxygen species. Mol Cell. 2012;48(2):158-67.

23. van der Vliet A. NADPH oxidases in lung biology and pathology: host defense enzymes, and more. Free Radic Biol Med. 2008;44(6):938-55.

24. Bueno M, Lai YC, Romero Y, Brands J, St Croix CM, Kamga C, et al. PINK1 deficiency impairs mitochondrial homeostasis and promotes lung fibrosis. J Clin Invest. 2015;125(2):521-38.

25. Zank DC, Bueno M, Mora AL, Rojas M. Idiopathic Pulmonary Fibrosis: Aging, Mitochondrial Dysfunction, and Cellular Bioenergetics. Front Med (Lausanne). 2018;5:10.

26. Hecker L, Vittal R, Jones T, Jagirdar R, Luckhardt TR, Horowitz JC, et al. NADPH oxidase-4 mediates myofibroblast activation and fibrogenic responses to lung injury. Nat Med. 2009;15(9):1077-81.

27. Jiang F, Liu GS, Dusting GJ, Chan EC. NADPH oxidase-dependent redox signaling in TGFbeta-mediated fibrotic responses. Redox Biol. 2014;2:267-72.

28. Bernard K, Logsdon NJ, Miguel V, Benavides GA, Zhang J, Carter AB, et al. NADPH Oxidase 4 (Nox4) Suppresses Mitochondrial Biogenesis and Bioenergetics in Lung Fibroblasts via a Nuclear Factor Erythroid-derived 2-like 2 (Nrf2)-dependent Pathway. J Biol Chem. 2017;292(7):3029-38. 29. American Thoracic Society. Idiopathic pulmonary fibrosis: diagnosis and treatment. International consensus statement. American Thoracic Society (ATS), and the European Respiratory Society (ERS). Am J Respir Crit Care Med. 2000;161(2 Pt 1):646-64.

30. Raghu G, Weycker D, Edelsberg J, Bradford WZ, Oster G. Incidence and prevalence of idiopathic pulmonary fibrosis. Am J Respir Crit Care Med. 2006;174(7):810-6.

31. Nalysnyk L, Cid-Ruzafa J, Rotella P, Esser D. Incidence and prevalence of idiopathic pulmonary fibrosis: review of the literature. Eur Respir Rev. 2012;21(126):355-61.

32. Navaratnam V, Fleming KM, West J, Smith CJ, Jenkins RG, Fogarty A, et al. The rising incidence of idiopathic pulmonary fibrosis in the U.K. Thorax. 2011;66(6):462-7.

33. Raghu G, Chen SY, Hou Q, Yeh WS, Collard HR. Incidence and prevalence of idiopathic pulmonary fibrosis in US adults 18-64 years old. The European respiratory journal. 2016;48(1):17986.

34. Mora AL, Rojas M, Pardo A, Selman M. Emerging therapies for idiopathic pulmonary fibrosis, a progressive age-related disease. Nature reviews Drug discovery. 2017;16(11):810.

35. Evans CM, Fingerlin TE, Schwarz MI, Lynch D, Kurche J, Warg L, et al. Idiopathic Pulmonary Fibrosis: A Genetic Disease That Involves Mucociliary Dysfunction of the Peripheral Airways. Physiol Rev. 2016;96(4):1567-91.

36. Cool CD, Groshong SD, Rai PR, Henson PM, Stewart JS, Brown KK. Fibroblast foci are not discrete sites of lung injury or repair: the fibroblast reticulum. Am J Respir Crit Care Med. 2006;174(6):654-8.

37. Sisson TH, Mendez M, Choi K, Subbotina N, Courey A, Cunningham A, et al. Targeted injury of type II alveolar epithelial cells induces pulmonary fibrosis. American journal of respiratory and critical care medicine. 2010;181(3):254-63.

38. Schafer MJ, White TA, lijima K, Haak AJ, Ligresti G, Atkinson EJ, et al. Cellular senescence mediates fibrotic pulmonary disease. Nat Commun. 2017;8:14532.

39. Selman M, Pardo A. Revealing the pathogenic and aging-related mechanisms of the enigmatic idiopathic pulmonary fibrosis. an integral model. American journal of respiratory and critical care medicine. 2014;189(10):1161-72.

40. Selman M, King TE, Pardo A, American Thoracic S, European Respiratory S, American College of Chest P. Idiopathic pulmonary fibrosis: prevailing and evolving hypotheses about its pathogenesis and implications for therapy. Ann Intern Med. 2001;134(2):136-51.

41. Plataki M, Koutsopoulos AV, Darivianaki K, Delides G, Siafakas NM, Bouros D. Expression of apoptotic and antiapoptotic markers in epithelial cells in idiopathic pulmonary fibrosis. Chest. 2005;127(1):266-74. 
42. Kuwano K, Kunitake R, Maeyama T, Hagimoto N, Kawasaki M, Matsuba T, et al. Attenuation of bleomycin-induced pneumopathy in mice by a caspase inhibitor. Am J Physiol Lung Cell Mol Physiol. 2001;280(2):L316-25.

43. Uhal BD, Joshi I, Hughes WF, Ramos C, Pardo A, Selman M. Alveolar epithelial cell death adjacent to underlying myofibroblasts in advanced fibrotic human lung. Am J Physiol. 1998;275(6 Pt 1):L1192-9.

44. Kasper M, Barth K. Potential contribution of alveolar epithelial type I cells to pulmonary fibrosis. Biosci Rep. 2017;37(6).

45. Abbadie $C$, Pluquet $O$, Pourtier A. Epithelial cell senescence: an adaptive response to precarcinogenic stresses? Cell Mol Life Sci. 2017;74(24):4471-509.

46. Kage $\mathrm{H}$, Borok Z. EMT and interstitial lung disease: a mysterious relationship. Curr Opin Pulm Med. 2012;18(5):517-23.

47. Jolly MK, Ward C, Eapen MS, Myers S, Hallgren O, Levine H, et al. Epithelial-mesenchymal transition, a spectrum of states: Role in lung development, homeostasis, and disease. Dev Dyn. 2018;247(3):346-58.

48. Kim KK, Kugler MC, Wolters PJ, Robillard L, Galvez MG, Brumwell AN, et al. Alveolar epithelial cell mesenchymal transition develops in vivo during pulmonary fibrosis and is regulated by the extracellular matrix. Proc Natl Acad Sci U S A. 2006;103(35):13180-5.

49. Willis BC, Liebler JM, Luby-Phelps K, Nicholson AG, Crandall ED, du Bois RM, et al. Induction of epithelial-mesenchymal transition in alveolar epithelial cells by transforming growth factor-beta1: potential role in idiopathic pulmonary fibrosis. The American journal of pathology. 2005;166(5):1321-32.

50. Rock JR, Barkauskas CE, Cronce MJ, Xue Y, Harris JR, Liang J, et al. Multiple stromal populations contribute to pulmonary fibrosis without evidence for epithelial to mesenchymal transition. Proc Natl Acad Sci U S A. 2011;108(52):E1475-83.

51. Camelo A, Dunmore R, Sleeman MA, Clarke DL. The epithelium in idiopathic pulmonary fibrosis: breaking the barrier. Frontiers in pharmacology. 2014;4:173.

52. Thannickal VJ, Lee DY, White ES, Cui Z, Larios JM, Chacon R, et al. Myofibroblast differentiation by transforming growth factor-beta1 is dependent on cell adhesion and integrin signaling via focal adhesion kinase. The Journal of biological chemistry. 2003;278(14):12384-9.

53. Hinz B, Phan SH, Thannickal VJ, Galli A, Bochaton-Piallat ML, Gabbiani G. The myofibroblast: one function, multiple origins. The American journal of pathology. 2007;170(6):180716.

54. Prasad S, Hogaboam CM, Jarai G. Deficient repair response of IPF fibroblasts in a coculture model of epithelial injury and repair. Fibrogenesis Tissue Repair. 2014;7:7.

55. Pardo A, Selman M. Idiopathic pulmonary fibrosis: new insights in its pathogenesis. Int J Biochem Cell Biol. 2002;34(12):1534-8.

56. Nicholson AG, Fulford LG, Colby TV, du Bois RM, Hansell DM, Wells AU. The relationship between individual histologic features and disease progression in idiopathic pulmonary fibrosis. Am J Respir Crit Care Med. 2002;166(2):173-7.

57. King TE, Jr., Schwarz MI, Brown K, Tooze JA, Colby TV, Waldron JA, Jr., et al. Idiopathic pulmonary fibrosis: relationship between histopathologic features and mortality. American journal of respiratory and critical care medicine. 2001;164(6):1025-32.

58. Yanai H, Shteinberg A, Porat Z, Budovsky A, Braiman A, Ziesche R, et al. Cellular senescence-like features of lung fibroblasts derived from idiopathic pulmonary fibrosis patients. Aging (Albany NY). 2015;7(9):664-72.

59. Gauldie J, Kolb M, Sime PJ. A new direction in the pathogenesis of idiopathic pulmonary fibrosis? Respir Res. 2002;3:1.

60. Jones HA, Schofield JB, Krausz T, Boobis AR, Haslett C. Pulmonary fibrosis correlates with duration of tissue neutrophil activation. Am J Respir Crit Care Med. 1998;158(2):620-8. 
61. Kinder BW, Brown KK, Schwarz MI, Ix JH, Kervitsky A, King TE, Jr. Baseline BAL neutrophilia predicts early mortality in idiopathic pulmonary fibrosis. Chest. 2008;133(1):226-32.

62. Wynn TA, Vannella KM. Macrophages in Tissue Repair, Regeneration, and Fibrosis. Immunity. 2016;44(3):450-62.

63. Xu X, Dai H, Wang C. Epithelium-dependent profibrotic milieu in the pathogenesis of idiopathic pulmonary fibrosis: current status and future directions. Clin Respir J. 2016;10(2):133-41. 64. Furuie $H$, Yamasaki $H$, Suga $M$, Ando M. Altered accessory cell function of alveolar macrophages: a possible mechanism for induction of Th2 secretory profile in idiopathic pulmonary fibrosis. Eur Respir J. 1997;10(4):787-94.

65. Barron L, Wynn TA. Fibrosis is regulated by Th2 and Th17 responses and by dynamic interactions between fibroblasts and macrophages. Am J Physiol Gastrointest Liver Physiol. 2011;300(5):G723-8.

66. Ryan US, Ryan JW. Cell biology of pulmonary endothelium. Circulation. 1984;70(5 Pt 2):III46-62.

67. Magro CM, Waldman WJ, Knight DA, Allen JN, Nadasdy T, Frambach GE, et al. Idiopathic pulmonary fibrosis related to endothelial injury and antiendothelial cell antibodies. Hum Immunol. 2006;67(4-5):284-97.

68. Takabatake N, Arao T, Sata M, Abe S, Inoue S, Shibata Y, et al. Involvement of pulmonary endothelial cell injury in the pathogenesis of pulmonary fibrosis: clinical assessment by 123I-MIBG lung scintigraphy. Eur J Nucl Med Mol Imaging. 2005;32(2):221-8.

69. Kato S, Inui N, Hakamata A, Suzuki Y, Enomoto N, Fujisawa T, et al. Changes in pulmonary endothelial cell properties during bleomycin-induced pulmonary fibrosis. Respir Res. 2018;19(1):127.

70. Farkas L, Farkas D, Ask K, Moller A, Gauldie J, Margetts P, et al. VEGF ameliorates pulmonary hypertension through inhibition of endothelial apoptosis in experimental lung fibrosis in rats. J Clin Invest. 2009;119(5):1298-311.

71. Malli F, Koutsokera A, Paraskeva E, Zakynthinos E, Papagianni M, Makris D, et al. Endothelial progenitor cells in the pathogenesis of idiopathic pulmonary fibrosis: an evolving concept. PLoS One. 2013;8(1):e53658.

72. Leach HG, Chrobak I, Han R, Trojanowska M. Endothelial cells recruit macrophages and contribute to a fibrotic milieu in bleomycin lung injury. Am J Respir Cell Mol Biol. 2013;49(6):1093101.

73. Piera-Velazquez S, Li Z, Jimenez SA. Role of endothelial-mesenchymal transition (EndoMT) in the pathogenesis of fibrotic disorders. The American journal of pathology. 2011;179(3):1074-80.

74. Hashimoto N, Phan SH, Imaizumi K, Matsuo M, Nakashima H, Kawabe T, et al. Endothelialmesenchymal transition in bleomycin-induced pulmonary fibrosis. Am J Respir Cell Mol Biol. 2010;43(2):161-72.

75. Lettieri CJ, Nathan SD, Barnett SD, Ahmad S, Shorr AF. Prevalence and outcomes of pulmonary arterial hypertension in advanced idiopathic pulmonary fibrosis. Chest. 2006;129(3):74652.

76. Kolb M, Bonella F, Wollin L. Therapeutic targets in idiopathic pulmonary fibrosis. Respiratory medicine. 2017;131:49-57.

77. Misra HP, Rabideau C. Pirfenidone inhibits NADPH-dependent microsomal lipid peroxidation and scavenges hydroxyl radicals. Mol Cell Biochem. 2000;204(1-2):119-26.

78. Giri SN, Leonard S, Shi X, Margolin SB, Vallyathan V. Effects of pirfenidone on the generation of reactive oxygen species in vitro. J Environ Pathol Toxicol Oncol. 1999;18(3):169-77. 79. Oku H, Shimizu T, Kawabata T, Nagira M, Hikita I, Ueyama A, et al. Antifibrotic action of pirfenidone and prednisolone: different effects on pulmonary cytokines and growth factors in bleomycin-induced murine pulmonary fibrosis. Eur J Pharmacol. 2008;590(1-3):400-8. 
80. Conte $\mathrm{E}$, Gili E, Fagone $\mathrm{E}$, Fruciano M, lemmolo M, Vancheri C. Effect of pirfenidone on proliferation, TGF-beta-induced myofibroblast differentiation and fibrogenic activity of primary human lung fibroblasts. Eur J Pharm Sci. 2014;58:13-9.

81. Hostettler KE, Zhong J, Papakonstantinou E, Karakiulakis G, Tamm M, Seidel P, et al. Antifibrotic effects of nintedanib in lung fibroblasts derived from patients with idiopathic pulmonary fibrosis. Respir Res. 2014;15:157.

82. Ciencewicki J, Trivedi S, Kleeberger SR. Oxidants and the pathogenesis of lung diseases. J Allergy Clin Immunol. 2008;122(3):456-68; quiz 69-70.

83. Bedard K, Krause KH. The NOX family of ROS-generating NADPH oxidases: physiology and pathophysiology. Physiol Rev. 2007;87(1):245-313.

84. Comhair SA, Erzurum SC. Antioxidant responses to oxidant-mediated lung diseases. Am J Physiol Lung Cell Mol Physiol. 2002;283(2):L246-55.

85. Valko M, Leibfritz D, Moncol J, Cronin MT, Mazur M, Telser J. Free radicals and antioxidants in normal physiological functions and human disease. The international journal of biochemistry \& cell biology. 2007;39(1):44-84.

86. Gonzalez-Gonzalez FJ, Chandel NS, Jain M, Budinger GRS. Reactive oxygen species as signaling molecules in the development of lung fibrosis. Transl Res. 2017;190:61-8.

87. Cantin AM, North SL, Fells GA, Hubbard RC, Crystal RG. Oxidant-mediated epithelial cell injury in idiopathic pulmonary fibrosis. The Journal of clinical investigation. 1987;79(6):1665-73.

88. Rahman I, Skwarska E, Henry M, Davis M, O'Connor CM, FitzGerald MX, et al. Systemic and pulmonary oxidative stress in idiopathic pulmonary fibrosis. Free Radic Biol Med. 1999;27(1-2):60-8.

89. Rottoli P, Magi B, Cianti R, Bargagli E, Vagaggini C, Nikiforakis N, et al. Carbonylated proteins in bronchoalveolar lavage of patients with sarcoidosis, pulmonary fibrosis associated with systemic sclerosis and idiopathic pulmonary fibrosis. Proteomics. 2005;5(10):2612-8.

90. Saleh D, Barnes PJ, Giaid A. Increased production of the potent oxidant peroxynitrite in the lungs of patients with idiopathic pulmonary fibrosis. American journal of respiratory and critical care medicine. 1997;155(5):1763-9.

91. Kuwano K, Nakashima N, Inoshima I, Hagimoto N, Fujita M, Yoshimi M, et al. Oxidative stress in lung epithelial cells from patients with idiopathic interstitial pneumonias. The European respiratory journal. 2003;21(2):232-40.

92. Fois AG, Paliogiannis $P$, Sotgia S, Mangoni AA, Zinellu E, Pirina $P$, et al. Evaluation of oxidative stress biomarkers in idiopathic pulmonary fibrosis and therapeutic applications: a systematic review. Respir Res. 2018;19(1):51.

93. Cantin AM, Hubbard RC, Crystal RG. Glutathione deficiency in the epithelial lining fluid of the lower respiratory tract in idiopathic pulmonary fibrosis. Am Rev Respir Dis. 1989;139(2):370-2.

94. Meyer A, Buhl R, Magnussen $\mathrm{H}$. The effect of oral N-acetylcysteine on lung glutathione levels in idiopathic pulmonary fibrosis. The European respiratory journal. 1994;7(3):431-6.

95. Veith C, Drent M, Bast A, van Schooten FJ, Boots AW. The disturbed redox-balance in pulmonary fibrosis is modulated by the plant flavonoid quercetin. Toxicol Appl Pharmacol. 2017;336:40-8.

96. Muramatsu Y, Sugino K, Ishida F, Tatebe J, Morita T, Homma S. Effect of inhaled Nacetylcysteine monotherapy on lung function and redox balance in idiopathic pulmonary fibrosis. Respir Investig. 2016;54(3):170-8.

97. Beeh KM, Beier J, Haas IC, Kornmann O, Micke P, Buhl R. Glutathione deficiency of the lower respiratory tract in patients with idiopathic pulmonary fibrosis. Eur Respir J. 2002;19(6):111923.

98. Kinnula VL, Crapo JD. Superoxide dismutases in the lung and human lung diseases. Am J Respir Crit Care Med. 2003;167(12):1600-19. 
99. Kinnula VL, Hodgson UA, Lakari EK, Tan RJ, Sormunen RT, Soini YM, et al. Extracellular superoxide dismutase has a highly specific localization in idiopathic pulmonary fibrosis/usual interstitial pneumonia. Histopathology. 2006;49(1):66-74.

100. Fattman CL, Chang LY, Termin TA, Petersen L, Enghild JJ, Oury TD. Enhanced bleomycininduced pulmonary damage in mice lacking extracellular superoxide dismutase. Free Radic Biol Med. 2003;35(7):763-71.

101. Bowler RP, Nicks M, Warnick K, Crapo JD. Role of extracellular superoxide dismutase in bleomycin-induced pulmonary fibrosis. Am J Physiol Lung Cell Mol Physiol. 2002;282(4):L719-26.

102. Kliment CR, Oury TD. Oxidative stress, extracellular matrix targets, and idiopathic pulmonary fibrosis. Free Radic Biol Med. 2010;49(5):707-17.

103. Borzi RM, Grigolo B, Meliconi R, Fasano L, Sturani C, Fabbri M, et al. Elevated serum superoxide dismutase levels correlate with disease severity and neutrophil degranulation in idiopathic pulmonary fibrosis. Clin Sci (Lond). 1993;85(3):353-9.

104. He C, Murthy S, McCormick ML, Spitz DR, Ryan AJ, Carter AB. Mitochondrial Cu,Znsuperoxide dismutase mediates pulmonary fibrosis by augmenting $\mathrm{H} 2 \mathrm{O} 2$ generation. The Journal of biological chemistry. 2011;286(17):15597-607.

105. Herrera B, Murillo MM, Alvarez-Barrientos A, Beltran J, Fernandez M, Fabregat I. Source of early reactive oxygen species in the apoptosis induced by transforming growth factor-beta in fetal rat hepatocytes. Free Radic Biol Med. 2004;36(1):16-26.

106. Odajima N, Betsuyaku T, Nagai K, Moriyama C, Wang DH, Takigawa T, et al. The role of catalase in pulmonary fibrosis. Respir Res. 2010;11:183.

107. Tiitto L, Kaarteenaho-Wiik R, Sormunen R, Holmgren A, Paakko P, Soini $Y$, et al. Expression of the thioredoxin system in interstitial lung disease. J Pathol. 2003;201(3):363-70.

108. Vuorinen K, Ohlmeier S, Lepparanta O, Salmenkivi K, Myllarniemi M, Kinnula VL. Peroxiredoxin II expression and its association with oxidative stress and cell proliferation in human idiopathic pulmonary fibrosis. J Histochem Cytochem. 2008;56(10):951-9.

109. Peltoniemi M, Kaarteenaho-Wiik R, Saily M, Sormunen R, Paakko P, Holmgren A, et al. Expression of glutaredoxin is highly cell specific in human lung and is decreased by transforming growth factor-beta in vitro and in interstitial lung diseases in vivo. Hum Pathol. 2004;35(8):1000-7. 110. Anathy V, Lahue KG, Chapman DG, Chia SB, Casey DT, Aboushousha R, et al. Reducing protein oxidation reverses lung fibrosis. Nat Med. 2018;24(8):1128-35.

111. Cho HY, Reddy SP, Kleeberger SR. Nrf2 defends the lung from oxidative stress. Antioxidants \& redox signaling. 2006;8(1-2):76-87.

112. Markart $\mathrm{P}$, Luboeinski T, Korfei M, Schmidt R, Wygrecka M, Mahavadi P, et al. Alveolar oxidative stress is associated with elevated levels of nonenzymatic low-molecular-weight antioxidants in patients with different forms of chronic fibrosing interstitial lung diseases. Antioxidants \& redox signaling. 2009;11(2):227-40.

113. Mazur W, Lindholm P, Vuorinen K, Myllarniemi M, Salmenkivi K, Kinnula VL. Cell-specific elevation of NRF2 and sulfiredoxin-1 as markers of oxidative stress in the lungs of idiopathic pulmonary fibrosis and non-specific interstitial pneumonia. Apmis. 2010;118(9):703-12.

114. Reddy NM, Kleeberger SR, Cho HY, Yamamoto M, Kensler TW, Biswal S, et al. Deficiency in Nrf2-GSH signaling impairs type II cell growth and enhances sensitivity to oxidants. Am J Respir Cell Mol Biol. 2007;37(1):3-8.

115. Kikuchi N, Ishii $Y$, Morishima $Y$, Yageta $Y$, Haraguchi N, Itoh K, et al. Nrf2 protects against pulmonary fibrosis by regulating the lung oxidant level and Th1/Th2 balance. Respir Res. 2010;11:31. 116. Zhang H, Davies KJA, Forman HJ. Oxidative stress response and Nrf2 signaling in aging. Free Radic Biol Med. 2015;88(Pt B):314-36.

117. Swamy SM, Rajasekaran NS, Thannickal VJ. Nuclear Factor-Erythroid-2-Related Factor 2 in Aging and Lung Fibrosis. Am J Pathol. 2016;186(7):1712-23. 
118. Zhou L, Zhang H, Davies KJA, Forman HJ. Aging-related decline in the induction of Nrf2regulated antioxidant genes in human bronchial epithelial cells. Redox biology. 2018;14:35-40.

119. Ray PD, Huang BW, Tsuji Y. Reactive oxygen species (ROS) homeostasis and redox regulation in cellular signaling. Cell Signal. 2012;24(5):981-90.

120. Droge W. Free radicals in the physiological control of cell function. Physiological reviews. 2002;82(1):47-95.

121. van der Vliet A, Janssen-Heininger YMW, Anathy V. Oxidative stress in chronic lung disease: From mitochondrial dysfunction to dysregulated redox signaling. Mol Aspects Med. 2018. 122. Thannickal VJ, Horowitz JC. Evolving concepts of apoptosis in idiopathic pulmonary fibrosis. Proc Am Thorac Soc. 2006;3(4):350-6.

123. Waghray M, Cui Z, Horowitz JC, Subramanian IM, Martinez FJ, Toews GB, et al. Hydrogen peroxide is a diffusible paracrine signal for the induction of epithelial cell death by activated myofibroblasts. FASEB J. 2005;19(7):854-6.

124. Waring $P$, Mullbacher A. Cell death induced by the Fas/Fas ligand pathway and its role in pathology. Immunol Cell Biol. 1999;77(4):312-7.

125. Anathy V, Roberson EC, Guala AS, Godburn KE, Budd RC, Janssen-Heininger YM. Redoxbased regulation of apoptosis: S-glutathionylation as a regulatory mechanism to control cell death. Antioxidants \& redox signaling. 2012;16(6):496-505.

126. Anathy V, Roberson E, Cunniff B, Nolin JD, Hoffman S, Spiess P, et al. Oxidative processing of latent Fas in the endoplasmic reticulum controls the strength of apoptosis. Mol Cell Biol. 2012;32(17):3464-78.

127. Fernandez IE, Eickelberg $\mathrm{O}$. The impact of TGF-beta on lung fibrosis: from targeting to biomarkers. Proceedings of the American Thoracic Society. 2012;9(3):111-6.

128. Thannickal VJ, Fanburg BL. Activation of an H2O2-generating NADH oxidase in human lung fibroblasts by transforming growth factor beta 1. J Biol Chem. 1995;270(51):30334-8.

129. Bellocq A, Azoulay E, Marullo S, Flahault A, Fouqueray B, Philippe C, et al. Reactive oxygen and nitrogen intermediates increase transforming growth factor-beta1 release from human epithelial alveolar cells through two different mechanisms. American journal of respiratory cell and molecular biology. 1999;21(1):128-36.

130. Liu RM, Desai LP. Reciprocal regulation of TGF-beta and reactive oxygen species: A perverse cycle for fibrosis. Redox Biol. 2015;6:565-77.

131. Lambeth JD. NOX enzymes and the biology of reactive oxygen. Nat Rev Immunol. 2004;4(3):181-9.

132. Martyn KD, Frederick LM, von Loehneysen K, Dinauer MC, Knaus UG. Functional analysis of Nox4 reveals unique characteristics compared to other NADPH oxidases. Cell Signal. 2006;18(1):69-

82.

133. Lambeth JD, Kawahara T, Diebold B. Regulation of Nox and Duox enzymatic activity and expression. Free Radic Biol Med. 2007;43(3):319-31.

134. Meitzler JL, Ortiz de Montellano PR. Caenorhabditis elegans and human dual oxidase 1 (DUOX1) "peroxidase" domains: insights into heme binding and catalytic activity. J Biol Chem. 2009;284(28):18634-43.

135. Kawahara T, Quinn MT, Lambeth JD. Molecular evolution of the reactive oxygengenerating NADPH oxidase (Nox/Duox) family of enzymes. BMC Evol Biol. 2007;7:109.

136. Bernard K, Hecker L, Luckhardt TR, Cheng G, Thannickal VJ. NADPH oxidases in lung health and disease. Antioxidants \& redox signaling. 2014;20(17):2838-53.

137. Choi SH, Kim M, Lee HJ, Kim EH, Kim CH, Lee YJ. Effects of NOX1 on fibroblastic changes of endothelial cells in radiationinduced pulmonary fibrosis. Mol Med Rep. 2016;13(5):4135-42.

138. Carnesecchi S, Deffert C, Pagano A, Garrido-Urbani S, Metrailler-Ruchonnet I, Schappi M, et al. NADPH oxidase-1 plays a crucial role in hyperoxia-induced acute lung injury in mice. Am J Respir Crit Care Med. 2009;180(10):972-81. 
139. Nauseef WM. How human neutrophils kill and degrade microbes: an integrated view. Immunol Rev. 2007;219:88-102.

140. Strausz J, Muller-Quernheim J, Steppling H, Ferlinz R. Oxygen radical production by alveolar inflammatory cells in idiopathic pulmonary fibrosis. Am Rev Respir Dis. 1990;141(1):124-8.

141. Wang CL, Kang J, Li ZH. [Increased expression of NADPH oxidase p47-PHOX and p67-PHOX factor in idiopathic pulmonary fibrosis]. Zhonghua Jie He He Hu Xi Za Zhi. 2007;30(4):265-8.

142. Manoury B, Nenan S, Leclerc O, Guenon I, Boichot E, Planquois JM, et al. The absence of reactive oxygen species production protects mice against bleomycin-induced pulmonary fibrosis. Respir Res. 2005;6:11.

143. Shvedova AA, Kisin ER, Murray AR, Kommineni C, Castranova V, Fadeel B, et al. Increased accumulation of neutrophils and decreased fibrosis in the lung of NADPH oxidase-deficient C57BL/6 mice exposed to carbon nanotubes. Toxicol Appl Pharmacol. 2008;231(2):235-40.

144. Singel KL, Segal BH. NOX2-dependent regulation of inflammation. Clin Sci (Lond). 2016;130(7):479-90.

145. Petry A, Djordjevic T, Weitnauer M, Kietzmann T, Hess J, Gorlach A. NOX2 and NOX4 mediate proliferative response in endothelial cells. Antioxidants \& redox signaling. 2006;8(910):1473-84.

146. Carnesecchi S, Deffert C, Donati Y, Basset O, Hinz B, Preynat-Seauve O, et al. A key role for NOX4 in epithelial cell death during development of lung fibrosis. Antioxidants \& redox signaling. 2011;15(3):607-19.

147. Amara N, Goven D, Prost F, Muloway R, Crestani B, Boczkowski J. NOX4/NADPH oxidase expression is increased in pulmonary fibroblasts from patients with idiopathic pulmonary fibrosis and mediates TGFbeta1-induced fibroblast differentiation into myofibroblasts. Thorax. 2010;65(8):733-8.

148. Cannito S, Novo E, di Bonzo LV, Busletta C, Colombatto S, Parola M. Epithelialmesenchymal transition: from molecular mechanisms, redox regulation to implications in human health and disease. Antioxidants \& redox signaling. 2010;12(12):1383-430.

149. Marmai C, Sutherland RE, Kim KK, Dolganov GM, Fang X, Kim SS, et al. Alveolar epithelial cells express mesenchymal proteins in patients with idiopathic pulmonary fibrosis. Am J Physiol Lung Cell Mol Physiol. 2011;301(1):L71-8.

150. Kalluri R, Neilson EG. Epithelial-mesenchymal transition and its implications for fibrosis. J Clin Invest. 2003;112(12):1776-84.

151. Boudreau HE, Casterline BW, Rada B, Korzeniowska A, Leto TL. Nox4 involvement in TGFbeta and SMAD3-driven induction of the epithelial-to-mesenchymal transition and migration of breast epithelial cells. Free Radic Biol Med. 2012;53(7):1489-99.

152. Hiraga R, Kato M, Miyagawa S, Kamata T. Nox4-derived ROS signaling contributes to TGFbeta-induced epithelial-mesenchymal transition in pancreatic cancer cells. Anticancer Res. 2013;33(10):4431-8.

153. Sanders YY, Liu H, Liu G, Thannickal VJ. Epigenetic mechanisms regulate NADPH oxidase-4 expression in cellular senescence. Free Radic Biol Med. 2015;79:197-205.

154. Pache JC, Carnesecchi S, Deffert C, Donati Y, Herrmann FR, Barazzone-Argiroffo C, et al. NOX-4 is expressed in thickened pulmonary arteries in idiopathic pulmonary fibrosis. Nat Med. 2011;17(1):31-2; author reply 2-3.

155. Sturrock A, Cahill B, Norman K, Huecksteadt TP, Hill K, Sanders K, et al. Transforming growth factor-beta1 induces Nox4 NAD(P)H oxidase and reactive oxygen species-dependent proliferation in human pulmonary artery smooth muscle cells. Am J Physiol Lung Cell Mol Physiol. 2006;290(4):L661-L73.

156. Jarman ER, Khambata VS, Cope C, Jones P, Roger J, Ye LY, et al. An inhibitor of NADPH oxidase- 4 attenuates established pulmonary fibrosis in a rodent disease model. Am J Respir Cell Mol Biol. 2014;50(1):158-69. 
157. Yan F, Wang Y, Wu X, Peshavariya HM, Dusting GJ, Zhang M, et al. Nox4 and redox signaling mediate TGF-beta-induced endothelial cell apoptosis and phenotypic switch. Cell Death Dis. 2014;5:e1010.

158. Kang KA, Ryu YS, Piao MJ, Shilnikova K, Kang HK, Yi JM, et al. DUOX2-mediated production of reactive oxygen species induces epithelial mesenchymal transition in 5-fluorouracil resistant human colon cancer cells. Redox biology. 2018;17:224-35.

159. Little AC, Sham D, Hristova M, Danyal K, Heppner DE, Bauer RA, et al. DUOX1 silencing in lung cancer promotes EMT, cancer stem cell characteristics and invasive properties. Oncogenesis. 2016;5(10):e261.

160. Rangarajan S, Bernard K, Thannickal VJ. Mitochondrial Dysfunction in Pulmonary Fibrosis. Ann Am Thorac Soc. 2017;14(Supplement_5):S383-S8.

161. Nicolson GL. Mitochondrial Dysfunction and Chronic Disease: Treatment With Natural Supplements. Integr Med (Encinitas). 2014;13(4):35-43.

162. Cloonan SM, Choi AM. Mitochondria in lung disease. J Clin Invest. 2016;126(3):809-20.

163. Liu X, Chen Z. The pathophysiological role of mitochondrial oxidative stress in lung diseases. J TransI Med. 2017;15(1):207.

164. Romano AD, Greco E, Vendemiale G, Serviddio G. Bioenergetics and mitochondrial dysfunction in aging: recent insights for a therapeutical approach. Curr Pharm Des.

2014;20(18):2978-92.

165. Gomes LC, Di Benedetto G, Scorrano L. During autophagy mitochondria elongate, are spared from degradation and sustain cell viability. Nat Cell Biol. 2011;13(5):589-98.

166. Palikaras K, Tavernarakis N. Mitochondrial homeostasis: the interplay between mitophagy and mitochondrial biogenesis. Exp Gerontol. 2014;56:182-8.

167. Sureshbabu A, Bhandari V. Targeting mitochondrial dysfunction in lung diseases: emphasis on mitophagy. Front Physiol. 2013;4:384.

168. Elmore S. Apoptosis: a review of programmed cell death. Toxicol Pathol. 2007;35(4):495-

516.

169. Kubli DA, Gustafsson AB. Mitochondria and mitophagy: the yin and yang of cell death control. Circ Res. 2012;111(9):1208-21.

170. Wu Z, Puigserver P, Andersson U, Zhang C, Adelmant G, Mootha V, et al. Mechanisms controlling mitochondrial biogenesis and respiration through the thermogenic coactivator PGC-1. Cell. 1999;98(1):115-24.

171. Baldelli S, Aquilano K, Ciriolo MR. Punctum on two different transcription factors regulated by PGC-1alpha: nuclear factor erythroid-derived 2-like 2 and nuclear respiratory factor 2 . Biochim Biophys Acta. 2013;1830(8):4137-46.

172. Brenmoehl J, Hoeflich A. Dual control of mitochondrial biogenesis by sirtuin 1 and sirtuin 3. Mitochondrion. 2013;13(6):755-61.

173. Reznick RM, Zong H, Li J, Morino K, Moore IK, Yu HJ, et al. Aging-associated reductions in AMP-activated protein kinase activity and mitochondrial biogenesis. Cell Metab. 2007;5(2):151-6.

174. Yu G, Tzouvelekis A, Wang R, Herazo-Maya JD, Ibarra GH, Srivastava A, et al. Thyroid hormone inhibits lung fibrosis in mice by improving epithelial mitochondrial function. Nat Med. 2018;24(1):39-49.

175. Lennon FE, Salgia R. Mitochondrial dynamics: biology and therapy in lung cancer. Expert Opin Investig Drugs. 2014;23(5):675-92.

176. Ashrafi G, Schwarz TL. The pathways of mitophagy for quality control and clearance of mitochondria. Cell Death Differ. 2013;20(1):31-42.

177. Sosulski ML, Gongora R, Danchuk S, Dong C, Luo F, Sanchez CG. Deregulation of selective autophagy during aging and pulmonary fibrosis: the role of TGFbeta1. Aging Cell. 2015;14(5):774-83. 178. Durcan TM, Fon EA. The three 'P's of mitophagy: PARKIN, PINK1, and post-translational modifications. Genes Dev. 2015;29(10):989-99. 
179. Patel AS, Lin L, Geyer A, Haspel JA, An CH, Cao J, et al. Autophagy in idiopathic pulmonary fibrosis. PLoS One. 2012;7(7):e41394.

180. Patel AS, Song JW, Chu SG, Mizumura K, Osorio JC, Shi Y, et al. Epithelial cell mitochondrial dysfunction and PINK1 are induced by transforming growth factor-beta1 in pulmonary fibrosis. PloS one. 2015;10(3):e0121246.

181. Panduri V, Liu G, Surapureddi S, Kondapalli J, Soberanes S, de Souza-Pinto NC, et al. Role of mitochondrial hOGG1 and aconitase in oxidant-induced lung epithelial cell apoptosis. Free Radic Biol Med. 2009;47(6):750-9.

182. Kim SJ, Cheresh P, Jablonski RP, Morales-Nebreda L, Cheng Y, Hogan E, et al. Mitochondrial catalase overexpressed transgenic mice are protected against lung fibrosis in part via preventing alveolar epithelial cell mitochondrial DNA damage. Free Radic Biol Med. 2016;101:482-90.

183. Jablonski RP, Kim SJ, Cheresh P, Williams DB, Morales-Nebreda L, Cheng Y, et al. SIRT3 deficiency promotes lung fibrosis by augmenting alveolar epithelial cell mitochondrial DNA damage and apoptosis. FASEB journal : official publication of the Federation of American Societies for Experimental Biology. 2017;31(6):2520-32.

184. Kim SJ, Cheresh P, Jablonski RP, Williams DB, Kamp DW. The Role of Mitochondrial DNA in Mediating Alveolar Epithelial Cell Apoptosis and Pulmonary Fibrosis. Int J Mol Sci. 2015;16(9):21486519.

185. Cheresh P, Morales-Nebreda L, Kim SJ, Yeldandi A, Williams DB, Cheng Y, et al. Asbestosinduced pulmonary fibrosis is augmented in 8-oxoguanine DNA glycosylase knockout mice.

American journal of respiratory cell and molecular biology. 2015;52(1):25-36.

186. Kim SJ, Cheresh P, Williams D, Cheng Y, Ridge K, Schumacker PT, et al. Mitochondriatargeted Ogg1 and aconitase-2 prevent oxidant-induced mitochondrial DNA damage in alveolar epithelial cells. The Journal of biological chemistry. 2014;289(9):6165-76.

187. Ellson CD, Dunmore R, Hogaboam CM, Sleeman MA, Murray LA. Danger-associated molecular patterns and danger signals in idiopathic pulmonary fibrosis. American journal of respiratory cell and molecular biology. 2014;51(2):163-8.

188. Zhong Z, Liang S, Sanchez-Lopez E, He F, Shalapour S, Lin XJ, et al. New mitochondrial DNA synthesis enables NLRP3 inflammasome activation. Nature. 2018;560(7717):198-203.

189. Ryu C, Sun H, Gulati M, Herazo-Maya JD, Chen Y, Osafo-Addo A, et al. Extracellular Mitochondrial DNA Is Generated by Fibroblasts and Predicts Death in Idiopathic Pulmonary Fibrosis. American journal of respiratory and critical care medicine. 2017;196(12):1571-81.

190. Li G, Yuzhen L, Yi C, Xiaoxiang C, Wei Z, Changqing Z, et al. DNasel protects against Paraquat-induced acute lung injury and pulmonary fibrosis mediated by mitochondrial DNA. Biomed Res Int. 2015;2015:386952.

191. Riteau N, Gasse P, Fauconnier L, Gombault A, Couegnat M, Fick L, et al. Extracellular ATP is a danger signal activating P2X7 receptor in lung inflammation and fibrosis. American journal of respiratory and critical care medicine. 2010;182(6):774-83.

192. Jain M, Rivera S, Monclus EA, Synenki L, Zirk A, Eisenbart J, et al. Mitochondrial reactive oxygen species regulate transforming growth factor-beta signaling. J Biol Chem. 2013;288(2):770-7.

193. Smeitink J, van den Heuvel L, DiMauro S. The genetics and pathology of oxidative phosphorylation. Nat Rev Genet. 2001;2(5):342-52.

194. Wong HS, Dighe PA, Mezera V, Monternier PA, Brand MD. Production of superoxide and hydrogen peroxide from specific mitochondrial sites under different bioenergetic conditions. The Journal of biological chemistry. 2017;292(41):16804-9.

195. Brand MD. Mitochondrial generation of superoxide and hydrogen peroxide as the source of mitochondrial redox signaling. Free Radic Biol Med. 2016;100:14-31.

196. Lee HC, Yin PH, Chi CW, Wei YH. Increase in mitochondrial mass in human fibroblasts under oxidative stress and during replicative cell senescence. J Biomed Sci. 2002;9(6 Pt 1):517-26. 
197. Yoon YS, Lee JH, Hwang SC, Choi KS, Yoon G. TGF beta1 induces prolonged mitochondrial ROS generation through decreased complex IV activity with senescent arrest in Mv1Lu cells. Oncogene. 2005;24(11):1895-903.

198. Santos JH, Hunakova L, Chen Y, Bortner C, Van Houten B. Cell sorting experiments link persistent mitochondrial DNA damage with loss of mitochondrial membrane potential and apoptotic cell death. J Biol Chem. 2003;278(3):1728-34.

199. Fang EF, Scheibye-Knudsen M, Chua KF, Mattson MP, Croteau DL, Bohr VA. Nuclear DNA damage signalling to mitochondria in ageing. Nat Rev Mol Cell Biol. 2016;17(5):308-21.

200. Guo Q. Changes in mitochondrial function during EMT induced by TGFbeta-1 in pancreatic cancer. Oncol Lett. 2017;13(3):1575-80.

201. Sato N, Takasaka N, Yoshida M, Tsubouchi K, Minagawa S, Araya J, et al. Metformin attenuates lung fibrosis development via NOX4 suppression. Respir Res. 2016;17(1):107.

202. Zandalinas SI, Mittler R. ROS-induced ROS release in plant and animal cells. Free Radic Biol Med. 2017.

203. Wosniak J, Jr., Santos CX, Kowaltowski AJ, Laurindo FR. Cross-talk between mitochondria and NADPH oxidase: effects of mild mitochondrial dysfunction on angiotensin II-mediated increase in Nox isoform expression and activity in vascular smooth muscle cells. Antioxidants \& redox signaling. 2009;11(6):1265-78.

204. Doughan AK, Harrison DG, Dikalov SI. Molecular mechanisms of angiotensin II-mediated mitochondrial dysfunction: linking mitochondrial oxidative damage and vascular endothelial dysfunction. Circ Res. 2008;102(4):488-96.

205. Kim YM, Kim SJ, Tatsunami R, Yamamura H, Fukai T, Ushio-Fukai M. ROS-induced ROS release orchestrated by Nox4, Nox2, and mitochondria in VEGF signaling and angiogenesis. Am J Physiol Cell Physiol. 2017;312(6):C749-C64.

206. Dikalov S. Cross talk between mitochondria and NADPH oxidases. Free Radic Biol Med. 2011;51(7):1289-301.

207. Boden WE, Padala SK, Cabral KP, Buschmann IR, Sidhu MS. Role of short-acting nitroglycerin in the management of ischemic heart disease. Drug Des Devel Ther. 2015;9:4793-805.

208. Wenzel P, Mollnau H, Oelze M, Schulz E, Wickramanayake JM, Muller J, et al. First evidence for a crosstalk between mitochondrial and NADPH oxidase-derived reactive oxygen species in nitroglycerin-triggered vascular dysfunction. Antioxidants \& redox signaling. 2008;10(8):1435-47.

209. Rey FE, Cifuentes ME, Kiarash A, Quinn MT, Pagano PJ. Novel competitive inhibitor of $\mathrm{NAD}(\mathrm{P}) \mathrm{H}$ oxidase assembly attenuates vascular $\mathrm{O}(2)(-)$ and systolic blood pressure in mice. Circ Res. 2001;89(5):408-14.

210. Rathore R, Zheng YM, Niu CF, Liu QH, Korde A, Ho YS, et al. Hypoxia activates NADPH oxidase to increase [ROS]i and [Ca2+]i through the mitochondrial ROS-PKCepsilon signaling axis in pulmonary artery smooth muscle cells. Free Radic Biol Med. 2008;45(9):1223-31.

211. Kroller-Schon S, Steven S, Kossmann S, Scholz A, Daub S, Oelze M, et al. Molecular mechanisms of the crosstalk between mitochondria and NADPH oxidase through reactive oxygen species-studies in white blood cells and in animal models. Antioxidants \& redox signaling. 2014;20(2):247-66.

212. Dikalova AE, Bikineyeva AT, Budzyn K, Nazarewicz RR, McCann L, Lewis W, et al. Therapeutic targeting of mitochondrial superoxide in hypertension. Circ Res. 2010;107(1):106-16. 213. Lee SB, Bae IH, Bae YS, Um HD. Link between mitochondria and NADPH oxidase 1 isozyme for the sustained production of reactive oxygen species and cell death. J Biol Chem. 2006;281(47):36228-35.

214. Graham KA, Kulawiec M, Owens KM, Li X, Desouki MM, Chandra D, et al. NADPH oxidase 4 is an oncoprotein localized to mitochondria. Cancer Biol Ther. 2010;10(3):223-31.

215. Block K, Gorin Y, Abboud HE. Subcellular localization of Nox4 and regulation in diabetes. Proc Natl Acad Sci U S A. 2009;106(34):14385-90. 
216. Kuroda J, Ago T, Matsushima S, Zhai P, Schneider MD, Sadoshima J. NADPH oxidase 4 (Nox4) is a major source of oxidative stress in the failing heart. Proc Natl Acad Sci U S A. 2010;107(35):15565-70.

217. Matsushima S, Kuroda J, Zhai P, Liu T, Ikeda S, Nagarajan N, et al. Tyrosine kinase FYN negatively regulates NOX4 in cardiac remodeling. J Clin Invest. 2016;126(9):3403-16.

218. Yu Q, Lee CF, Wang W, Karamanlidis G, Kuroda J, Matsushima S, et al. Elimination of NADPH oxidase activity promotes reductive stress and sensitizes the heart to ischemic injury. J Am Heart Assoc. 2014;3(1):e000555.

219. Ogura M, Yamaki J, Homma MK, Homma Y. Mitochondrial c-Src regulates cell survival through phosphorylation of respiratory chain components. The Biochemical journal.

2012;447(2):281-9.

220. Zhang H, Davies KJ, Forman HJ. TGFbeta1 rapidly activates Src through a non-canonical redox signaling mechanism. Arch Biochem Biophys. 2015;568:1-7.

221. Koziel R, Pircher H, Kratochwil M, Lener B, Hermann M, Dencher NA, et al. Mitochondrial respiratory chain complex $I$ is inactivated by NADPH oxidase Nox4. Biochem J. 2013;452(2):231-9.

222. Kim SM, Kim YG, Jeong KH, Lee SH, Lee TW, Ihm CG, et al. Angiotensin II-induced mitochondrial Nox4 is a major endogenous source of oxidative stress in kidney tubular cells. PLoS One. 2012;7(7):e39739.

223. Kang YP, Lee SB, Lee JM, Kim HM, Hong JY, Lee WJ, et al. Metabolic Profiling Regarding Pathogenesis of Idiopathic Pulmonary Fibrosis. J Proteome Res. 2016;15(5):1717-24.

224. Lyle AN, Deshpande NN, Taniyama Y, Seidel-Rogol B, Pounkova L, Du P, et al. Poldip2, a novel regulator of Nox4 and cytoskeletal integrity in vascular smooth muscle cells. Circ Res. 2009;105(3):249-59.

225. Cheng X, Kanki T, Fukuoh A, Ohgaki K, Takeya R, Aoki Y, et al. PDIP38 associates with proteins constituting the mitochondrial DNA nucleoid. J Biochem. 2005;138(6):673-8.

226. Shanmugasundaram K, Nayak BK, Friedrichs WE, Kaushik D, Rodriguez R, Block K. NOX4 functions as a mitochondrial energetic sensor coupling cancer metabolic reprogramming to drug resistance. Nat Commun. 2017;8(1):997.

227. Xie N, Tan Z, Banerjee S, Cui H, Ge J, Liu RM, et al. Glycolytic Reprogramming in Myofibroblast Differentiation and Lung Fibrosis. Am J Respir Crit Care Med. 2015;192(12):1462-74. 228. Maher TM. Aerobic Glycolysis and the Warburg Effect. An Unexplored Realm in the Search for Fibrosis Therapies? Am J Respir Crit Care Med. 2015;192(12):1407-9.

229. Sugiura H, Ichikawa T, Liu X, Kobayashi T, Wang XQ, Kawasaki S, et al. N-acetyl-L-cysteine inhibits TGF-beta1-induced profibrotic responses in fibroblasts. Pulm Pharmacol Ther. 2009;22(6):487-91.

230. Hagiwara SI, Ishii $\mathrm{Y}$, Kitamura S. Aerosolized administration of $\mathrm{N}$-acetylcysteine attenuates lung fibrosis induced by bleomycin in mice. Am J Respir Crit Care Med. 2000;162(1):225-31.

231. Cortijo J, Cerda-Nicolas M, Serrano A, Bioque G, Estrela JM, Santangelo F, et al. Attenuation by oral $\mathrm{N}$-acetylcysteine of bleomycin-induced lung injury in rats. Eur Respir J. 2001;17(6):1228-35.

232. Rogliani P, Calzetta L, Cavalli F, Matera MG, Cazzola M. Pirfenidone, nintedanib and Nacetylcysteine for the treatment of idiopathic pulmonary fibrosis: A systematic review and metaanalysis. Pulm Pharmacol Ther. 2016;40:95-103.

233. Myllarniemi M, Kaarteenaho R. Pharmacological treatment of idiopathic pulmonary fibrosis - preclinical and clinical studies of pirfenidone, nintedanib, and $\mathrm{N}$-acetylcysteine. Eur Clin Respir J. 2015;2.

234. Martinez FJ, de Andrade JA, Anstrom KJ, King TE, Jr., Raghu G. Randomized trial of acetylcysteine in idiopathic pulmonary fibrosis. The New England journal of medicine.

2014;370(22):2093-101. 
235. Okuda R, Matsushima H, Oba T, Kawabe R, Matsubayashi M, Amano M, et al. Efficacy and safety of inhaled $\mathrm{N}$-acetylcysteine in idiopathic pulmonary fibrosis: A prospective, single-arm study. Respir Investig. 2016;54(3):156-61.

236. Homma S, Azuma A, Taniguchi H, Ogura T, Mochiduki Y, Sugiyama Y, et al. Efficacy of inhaled $\mathrm{N}$-acetylcysteine monotherapy in patients with early stage idiopathic pulmonary fibrosis. Respirology. 2012;17(3):467-77.

237. Idiopathic Pulmonary Fibrosis Clinical Research N, Martinez FJ, de Andrade JA, Anstrom KJ, King TE, Jr., Raghu G. Randomized trial of acetylcysteine in idiopathic pulmonary fibrosis. N Engl J Med. 2014;370(22):2093-101.

238. Demedts M, Behr J, Buhl R, Costabel U, Dekhuijzen R, Jansen HM, et al. High-dose acetylcysteine in idiopathic pulmonary fibrosis. N Engl J Med. 2005;353(21):2229-42.

239. Sakamoto S, Itoh T, Muramatsu Y, Satoh K, Ishida F, Sugino K, et al. Efficacy of pirfenidone in patients with advanced-stage idiopathic pulmonary fibrosis. Intern Med. 2013;52(22):2495-501.

240. Gharaee-Kermani M, Moore BB, Macoska JA. Resveratrol-Mediated Repression and Reversion of Prostatic Myofibroblast Phenoconversion. PLoS One. 2016;11(7):e0158357.

241. Giovannelli L, Pitozzi V, Jacomelli M, Mulinacci N, Laurenzana A, Dolara P, et al. Protective effects of resveratrol against senescence-associated changes in cultured human fibroblasts. J Gerontol A Biol Sci Med Sci. 2011;66(1):9-18.

242. Nakamura T, Matsushima M, Hayashi Y, Shibasaki M, Imaizumi K, Hashimoto N, et al. Attenuation of transforming growth factor-beta-stimulated collagen production in fibroblasts by quercetin-induced heme oxygenase-1. Am J Respir Cell Mol Biol. 2011;44(5):614-20.

243. Verma R, Kushwah L, Gohel D, Patel M, Marvania T, Balakrishnan S. Evaluating the Ameliorative Potential of Quercetin against the Bleomycin-Induced Pulmonary Fibrosis in Wistar Rats. Pulm Med. 2013;2013:921724.

244. He X, Wang L, Szklarz G, Bi Y, Ma Q. Resveratrol inhibits paraquat-induced oxidative stress and fibrogenic response by activating the nuclear factor erythroid 2-related factor 2 pathway. J Pharmacol Exp Ther. 2012;342(1):81-90.

245. Sener G, Topaloglu N, Sehirli AO, Ercan F, Gedik N. Resveratrol alleviates bleomycininduced lung injury in rats. Pulm Pharmacol Ther. 2007;20(6):642-9.

246. Akgedik R, Akgedik S, Karamanli H, Uysal S, Bozkurt B, Ozol D, et al. Effect of resveratrol on treatment of bleomycin-induced pulmonary fibrosis in rats. Inflammation. 2012;35(5):1732-41.

247. Schmidt HH, Stocker R, Vollbracht C, Paulsen G, Riley D, Daiber A, et al. Antioxidants in Translational Medicine. Antioxidants \& redox signaling. 2015;23(14):1130-43.

248. Hohmann MS, Habiel DM, Coelho AL, Verri WA, Jr., Hogaboam CM. Quercetin Enhances Ligand-induced Apoptosis in Senescent IPF Fibroblasts and Reduces Lung Fibrosis In Vivo. American journal of respiratory cell and molecular biology. 2018.

249. Zhu Y, Tchkonia T, Pirtskhalava T, Gower AC, Ding H, Giorgadze N, et al. The Achilles' heel of senescent cells: from transcriptome to senolytic drugs. Aging Cell. 2015;14(4):644-58.

250. Ghezzi P, Jaquet V, Marcucci F, Schmidt H. The oxidative stress theory of disease: levels of evidence and epistemological aspects. Br J Pharmacol. 2017;174(12):1784-96.

251. Kawagishi $\mathrm{H}$, Finkel T. Unraveling the truth about antioxidants: ROS and disease: finding the right balance. Nat Med. 2014;20(7):711-3.

252. Rehman H, Liu Q, Krishnasamy Y, Shi Z, Ramshesh VK, Haque K, et al. The mitochondriatargeted antioxidant MitoQ attenuates liver fibrosis in mice. Int J Physiol Pathophysiol Pharmacol. 2016;8(1):14-27.

253. Xun Z, Rivera-Sanchez S, Ayala-Pena S, Lim J, Budworth H, Skoda EM, et al. Targeting of XJB-5-131 to mitochondria suppresses oxidative DNA damage and motor decline in a mouse model of Huntington's disease. Cell Rep. 2012;2(5):1137-42. 
254. Polyzos A, Holt A, Brown C, Cosme C, Wipf P, Gomez-Marin A, et al. Mitochondrial targeting of XJB-5-131 attenuates or improves pathophysiology in HdhQ150 animals with welldeveloped disease phenotypes. Hum Mol Genet. 2016;25(9):1792-802.

255. Thakur S, Viswanadhapalli S, Kopp JB, Shi Q, Barnes JL, Block K, et al. Activation of AMPactivated protein kinase prevents TGF-beta1-induced epithelial-mesenchymal transition and myofibroblast activation. The American journal of pathology. 2015;185(8):2168-80.

256. Rangarajan S, Bone NB, Zmijewska AA, Jiang S, Park DW, Bernard K, et al. Metformin reverses established lung fibrosis in a bleomycin model. Nature medicine. 2018;24(8):1121-7. 257. Spagnolo P, Kreuter M, Maher TM, Wuyts W, Bonella F, Corte TJ, et al. Metformin Does Not Affect Clinically Relevant Outcomes in Patients with Idiopathic Pulmonary Fibrosis. Respiration. 2018:1-9.

258. Raghu G, Rochwerg B, Zhang Y, Garcia CA, Azuma A, Behr J, et al. An Official ATS/ERS/JRS/ALAT Clinical Practice Guideline: Treatment of Idiopathic Pulmonary Fibrosis. An Update of the 2011 Clinical Practice Guideline. Am J Respir Crit Care Med. 2015;192(2):e3-19. 259. Hayton C, Chaudhuri N. Managing Idiopathic Pulmonary Fibrosis: Which Drug for Which Patient? Drugs Aging. 2017;34(9):647-53. 




\section{Chapter 3}

\section{The disturbed redox-balance in pulmonary fibrosis is modulated by the plant flavonoid quercetin}

Carmen Veith, Marjolein Drent, Aalt Bast, Frederik-Jan van Schooten and Agnes W. Boots.

Toxicology and Applied Pharmacology 336 (2017): 40-48. 


\section{Abstract}

Idiopathic pulmonary fibrosis (IPF) is characterized by a disturbed pulmonary redox balance associated with inflammation. To restore this balance, antioxidants are often suggested as therapy for IPF but previous clinical trials with these compounds and their precursors have not been successful in the clinic. The exogenous antioxidant quercetin, which has a versatile antioxidant profile and is effective in restoring a disturbed redox balance, might be a better candidate. The aim of this study was to evaluate the protective effect of quercetin on oxidative and inflammatory markers in IPF. Here, we demonstrate that IPF patients have a significantly reduced endogenous antioxidant defence, shown by a reduced total antioxidant capacity and lowered glutathione and uric acid levels compared to healthy controls. This confirms that the redox balance is disturbed in IPF. Ex vivo incubation with quercetin in blood of both IPF patients and healthy controls reduces LPS-induced production of the pro-inflammatory cytokines IL-8 and TNF $\alpha$. This antiinflammatory effect was more pronounced in the blood of the patients. Our pro-fibrotic in vitro model, consisting of bleomycin-triggered BEAS-2B cells, shows that quercetin boosts the antioxidant response, by increasing Nrf2 activity, and decreases pro-inflammatory cytokine production in a concentration-dependent manner. Collectively, our findings implicate that IPF patients may benefit from the use of quercetin to restore the disturbed redox balance and reduce inflammation.

Keywords: Interstitial lung diseases (ILD), Idiopathic pulmonary fibrosis (IPF), oxidative stress, inflammation, antioxidants, quercetin 


\section{Introduction}

Idiopathic pulmonary fibrosis (IPF) is a chronic, progressive and lethal disease of unknown cause with a median survival of only 3-5 years which is worse than that of several types of cancer (e.g., breast, bladder, and colorectal) (1-3). It is the most common and severe form of idiopathic interstitial lung diseases that affects mainly 60- to 70-year old ever smokers (2). This disease is characterized by an exuberant and uncontrolled reparative process following chronic alveolar epithelial microinjuries, leading to excessive scarring of the lungs which impairs the ability of the lungs to transport oxygen $(4,5)$. While IPF is by definition "idiopathic" (i.e., of unknown cause), the list of potential fibrogenic triggers that have been associated with IPF includes, among others, cigarette smoking, exposure to man-made fibers and chronic infection (5). Key clinical feature is a cruelly impaired lung function, ultimately leading to death from respiratory failure (5). In the US only, IPF affects between $150,000-200,000$ people, and as many as 40,000 people die from this disease each year (6). Similar incidence, prevalence and mortality rates have been reported in Europe with for example a yearly incidence of approximately 1000-1500 new cases in the Netherlands $(7,8)$.

IPF is characterized by a disturbed pulmonary redox-balance due to a chronic overload of ROS in comparison to protective antioxidants $(9,10)$. The increased ROS production is suggested to play a critical role in epithelial activation and injury of the alveolar cells including damage to DNA, lipids and proteins which ultimately causes severe tissue damage thereby contributing to the progression of the disease (11-13). Moreover, increased ROS levels are critical in driving the progression of IPF mainly via triggering the activation and release of active TGF- $\beta 1$, that augments the existing inflammation and lung scarring (14). Furthermore, ROS mediate TGF- $\beta 1$-induced fibrogenesis including activation and proliferation of fibroblasts, differentiation into myofibroblasts causing secretion of ECM molecules and excessive collagen deposition, activation of pro-inflammatory cells and induction of EMT (15). Under normal circumstances, the endogenous antioxidant defense system provides sufficient protection against ROS (16) but in patients burdened with IPF this system may be overloaded. Part of the pulmonary antioxidant defense system is regulated by the redox sensitive transcription factor nuclear factor eryrhroid 2related factor 2 (Nrf2) (15). In the presence of oxidative stress, Nrf2 translocates into the nucleus where it will bind to the antioxidant response elements (ARE) that will induce the transcription of antioxidant genes (17). Nrf2 is upregulated in IPF to restore the pulmonary redox status but not to a sufficient enough level, leaving IPF patients exposed to elevated ROS levels (18). Consequently, antioxidant therapy to strengthen such a reduced antioxidant defense might be efficacious in IPF treatment. Since ROS are capable of initiating and mediating inflammation, antioxidant therapy might also mitigate the elevated pro-inflammatory response. 
Despite the fact that numerous large clinical trials have been conducted over the last few years since Raghu et al published the IPF guidelines for diagnosis and management, there is still no effective cure for IPF that increases survival (19). Consequently, current standard treatment still consists of anti-inflammatory and immunosuppressive agents, aiming at reducing symptoms and the underlying inflammation (2). Moreover, current IPF treatments such as pirfenidone and $\mathrm{N}$-acetyl-L-cysteine (NAC) aiming at decreasing oxidative stress in the lungs do show a clinical benefit but fail to be successful. The latest update of these guidelines therefore acknowledges the need for further research regarding the pathology of the disease as well as the efficacy and long-term safety of IPF medications to improve the outcome of the disease and the patient's quality of life (20). Alternatively, other strong antioxidants with a more versatile character, including those present in our daily diet, have already been suggested as good candidates for antioxidant therapy in interstitial lung diseases (ILD) including IPF (21). The dietary antioxidant quercetin $\left(3,3^{\prime}, 4^{\prime}, 5,7\right.$-pentahydroxyflavone) for instance is a prototypical polyphenolic plant flavonoid which is mainly present in vegetables and fruit as well as in tea and red wine (22). It is suggested that quercetin is the most potent scavenger of ROS, including superoxide, within the flavanoid family (22). By activating the Nrf2 pathway, it also indirectly exerts its function through induction of Nrf2-regulated genes to further increase specific protection against oxidants $(23,24)$. However, quercetin is not only known for its strong anti-oxidative but also for its anti-inflammatory capacities both in vitro $(25,26)$ and in various pulmonary models (27-29). Additionally, studies indicate that it is also effective in restoring a disturbed redox balance and reducing inflammation in vivo in other interstitial lung diseases (ILD) such as sarcoidosis $(30,31)$.

The aim of the present study is to evaluate the antioxidant and inflammatory status in IPF as well as the possible beneficial effects of the dietary antioxidant quercetin hereon. To this end, the protective effects of quercetin on the antioxidant defense and inflammatory markers have been determined both in vitro in pulmonary epithelial cells and ex vivo in LPS-stimulated blood of IPF patients. We hypothesize that the antioxidant and inflammatory status in IPF are compromised and that quercetin can offer protection against oxidant-induced lung damage by restoring the disturbed redox balance and decreasing levels of inflammatory markers. 


\section{Materials \& Methods}

\subsection{Clinical study}

Eleven non-smoking patients with IPF, diagnosed according to the guidelines (2), were enrolled in this study via recruitment in our outpatient clinic (Table 1). The control group, matched on age and gender, consisted of 9 non-smoking healthy controls. Based on food questionnaires, all patients and controls had comparable dietary habits with an average daily quercetin intake of $15 \mathrm{mg}$. The Medical Ethical Committee of the Maastricht University Medical Center had approved the protocol and the study was registered at http://www.clinicaltrials.gov (NCT-00512967). All participants have given their written informed consent.

Table 3.1: Patient characteristics: non-smoking IPF patients and healthy matched controls. Age is expressed in years, length in $\mathrm{cm}$, weight in $\mathrm{kg}$, time since diagnosis in years and DLCO (diffuse capacity of the lung for carbon monoxide), $\mathrm{FEV}_{1}$ (forced expiratory volume in $1 \mathrm{~s}$ ) and FVC (forced vital capacity) in percentage of the predicted value based on age and gender. Data are expressed as mean \pm SEM.

\begin{tabular}{|c|c|c|}
\hline & IPF patients & Matched controls \\
\hline Number (m/f) & $11(8 / 3)$ & $9(5 / 4)$ \\
\hline Age (years) & $39-74(63 \pm 3)$ & $45-60(57 \pm 3)$ \\
\hline Length $(\mathrm{cm})$ & $158-174(167 \pm 2)$ & $160-179(170 \pm 3)$ \\
\hline $\begin{array}{l}\text { Body mass index } \\
\qquad\left(\mathrm{kg} / \mathrm{m}^{2}\right)\end{array}$ & $18.5-31(27 \pm 1)$ & $18.5-30(26 \pm 1)$ \\
\hline Time since diagnosis (years) & $0-4(2 \pm 0,4)$ & - \\
\hline Biopsy taken & $7 / 11$ & - \\
\hline $\begin{array}{c}\text { DLCO } \\
\text { (percentage predicted) }\end{array}$ & $19-66(42 \pm 5)$ & - \\
\hline FEV1 (percentage) & $44-86(72 \pm 4)$ & - \\
\hline FVC (percentage) & $36-140(78 \pm 8)$ & - \\
\hline Medication & $\begin{array}{l}\text { NAC (1500 mg daily): 4x } \\
\text { Prednisone (30 mg daily): 1x } \\
\text { NAC (1500 mg daily) + } \\
\text { Prednisone (25-30 mg } \\
\text { daily): } 5 x\end{array}$ & - \\
\hline
\end{tabular}




\subsection{Lung function measurement}

Forced vital capacity (FVC) and forced expiratory volume in one second $\left(F_{E V}\right)$ were measured with a pneumotachograph (Masterlab, Jaeger, Würzburg, Germany). The diffusing capacity of the lung for carbon monoxide (DLCO) was measured using the singlebreath method (Masterlab) (32).

\subsection{Cell culture and treatments}

The human bronchial epithelial cell line BEAS-2B was obtained from the American Type Culture Collection (ATCC, Rockville, MD) and maintained in DMEM/F12 supplemented with cholera Toxin $(10 \mathrm{ng} / \mathrm{ml})$, epidermal growth factor $(10 \mathrm{ng} / \mathrm{mL})$, insulin $(5 \mu \mathrm{g} / \mathrm{mL})$, transferin $(5 \mu \mathrm{g} / \mathrm{mL})$, dexamethasone $(0.1 \mu \mathrm{M})$, bovine pituitary extract $(15 \mu \mathrm{g} / \mathrm{mL})$ and bovine serum albumin $(0.5 \mathrm{mg} / \mathrm{mL})$. Cells were pretreated for 30 min with 10,25 or $50 \mu \mathrm{M}$ quercetin (Sigma, St. Lois, MO) or $0.1 \%$ ethanol (vehicle control) and subsequently exposed to different concentrations $(0.1,0.3,1,3,10,30 \mu \mathrm{g} / \mathrm{mL}$ ) bleomycin for various time periods ( $2 \mathrm{~h}, 4 \mathrm{~h}, 24 \mathrm{~h}$ or $48 \mathrm{~h})$.

\subsection{Preparation of blood samples}

Blood was collected in EDTA-containing vacutainer tubes (Vacutainer, Becton-Dickinson, Franklin Lakes, NJ) and kept on ice prior to processing.

\subsection{Trolox antioxidant capacity}

The trolox equivalent antioxidant capacity (TEAC value) is a measurement for the total antioxidant status, assessing the capacity of a compound to scavenge ABTS radicals. This assay has been performed as described earlier (33) with some minor modifications. In short, blood was centrifuged (3000 rpm, 5' at 4 - C) and plasma was afterwards deproteinized, using 10\% TCA (1:1) followed by centrifugation (13.000 rpm, $5^{\prime}$ at $\left.40 \mathrm{C}\right)$. A solution of $0.23 \mathrm{mM}$ ABTS (2,2'-azinobis-(3-ethylbenzothiazoline-6-sulfonic acid)) and $2.3 \mathrm{mM}$ ABAP $\left(2,2^{\prime}\right.$-Azobis-(2-amidinopropane) $\left.\mathrm{HCl}\right)$ was incubated at $70^{\circ} \mathrm{C}$ to generate $A B T S$ radicals ( $\left(A B T S^{\circ}\right)$ until the absorbance at $734 \mathrm{~nm}$ reached $0.70 \pm 0.02$. To measure the antioxidant capacity of the samples, $50 \mu \mathrm{l}$ of the plasma sample was mixed with $950 \mu \mathrm{l}$ ABTS solution and incubated for $5 \mathrm{~min}$ at $37^{\circ} \mathrm{C}$. The decrease in absorbance was measured at $734 \mathrm{~nm}$ and related to the absorbance of trolox standards to calculate the trolox equivalent of the samples.

\subsection{Measurement of endogenous antioxidants}

\section{Vitamin C}

To assess Vitamin $\mathrm{C}$ in the blood, $10 \%$ TCA was added and blood samples were centrifuged (5000 rpm, 10'at $4 \circ \mathrm{C}$ ). Ascorbate oxidase (Roche Diagnostics, Basel, Switzerland) was added to the plasma samples, leading to the oxidation of vitamin $\mathrm{C}$ to dehydro-ascorbic acid. 0.1\% (w:v) O-phenyleendiamin (OPDA) (Sigma) was added and incubated for $30 \mathrm{~min}$ at $37^{\circ} \mathrm{C}$. HPLC analysis was performed using a Hypersil BDS column 96 
(125 mm x $2 \mathrm{~mm}$, particle size $5 \mu \mathrm{m}$ from Agilent, Palo Alto, CA) with excitation at $355 \mathrm{~nm}$ and emission at $425 \mathrm{~nm}$ and ascorbic acid content was quantified using calibrators.

\section{Uric acid measurement}

Shortly, deproteinated plasma samples was injected in the HPLC system with fluorescent detection. HPLC analysis was performed using a Hypersil BDS column (125 mm x $4 \mathrm{~mm}$, particle size $5 \mu \mathrm{m}$ from Agilent) with UV detection at $292 \mathrm{~nm}$. Uric acid content of each sample was quantified using calibrators with known levels of uric acid.

\section{GSH and GSSG}

Prior to storage, $1.3 \% \mathrm{SSA}$ in $10 \mathrm{mM} \mathrm{HCl}$ was added to the blood samples used for the determination of reduced glutathione (GSH) and oxidized glutathione (GSSG). All samples were centrifuged (5000 rpm, 10'at 4으) and supernatant was collected. For the GSH

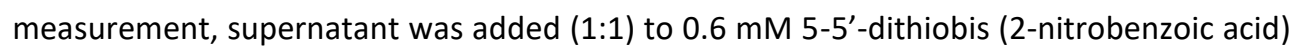
(DTNB) (Sigma) $\left(37^{\circ} \mathrm{C}\right)$ and incubated for $15 \mathrm{sec}\left(37^{\circ} \mathrm{C}\right)$, and absorbance was measured at $412 \mathrm{~nm}$. For the GSSG measurement, supernatant was added to 2-vinyl-pyridine (Sigma) (10:1) and incubated for 1 hour at room temperature. Subsequently, a mixture of $0.6 \mathrm{mM}$ DTNB and $0.8 \mathrm{mM} \mathrm{NADPH}\left(37^{\circ} \mathrm{C}\right)$ was added $(1: 9)$ and the reaction was started by adding the enzyme glutathione reductase (1:20). The absorbance was measured for $3 \mathrm{~min}$ at 412 $\mathrm{nm}$. Both the GSH and GSSG content of each sample were quantified using calibrators and related to the haemoglobin content.

\subsection{Blood-based cytokine production assay}

Within one hour after blood collection, the blood-based cytokine production assay was performed as described previously with minor modifications (34). Shortly, blood was diluted in RPMI 1640 (1:4, Gibco) and incubated with 1, 3 or $30 \mu \mathrm{M}$ quercetin for $30 \mathrm{~min}$. To induce cytokine production, lipopolysaccharide (LPS, E.coli 0.26:B6) (Sigma) was added and samples were incubated for 24 hours at $37^{\circ} \mathrm{C}$. Cell-free supernatant fluids were collected by centrifugation $\left(6000 \mathrm{rpm}, 10^{\prime}\right.$ at $4{ }^{\circ} \mathrm{C}$ ) and stored at $20^{\circ} \mathrm{C}$ prior to cytokine analysis.

\subsection{ELISA measurement}

Plasma and supernatant TNF $\alpha$ and IL-8 levels were quantified using a human ELISA kit according to the manufacturer's instructions (Sanquin, Amsterdam, the Netherlands).

\subsection{RNA isolation}

RNA was isolated and purified using the RNeasy mini kit (Qiagen, Venlo, the Netherlands) according to the manufacturer's instructions. The RNA concentration was determined using a Nanodrop spectrophotometer (Thermo Scientific, Waltham, MA). 


\section{$2.10 \quad$ RT-PCR}

cDNA was synthesized from 500 ng isolated RNA using IScript (Biorad, Hercules, CA) according to the manufacturer's instructions. RT-PCR was performed using SYBR Green PCR Supermix (BioRad) and PCR amplifications were carried out for 40 cycles of denaturation $\left(95^{\circ} \mathrm{C}, 15 \mathrm{sec}\right)$, annealing $\left(57^{\circ} \mathrm{C}, 15 \mathrm{sec}\right)$ and extension $\left(60^{\circ} \mathrm{C}, 45 \mathrm{sec}\right)$ for selected genes (Table 2). The gene expression was normalized to GAPDH using the $\Delta \Delta C_{T}$ method.

Table 3.2: $\underline{\text { Human RT-PCR forward and reverse primer sequences. }}$

\begin{tabular}{|l|l|l|}
\hline $\begin{array}{l}\text { Gene of } \\
\text { interest }\end{array}$ & Forward primer sequence & Reverse primer sequence \\
\hline Nrf2 & ACACGGTCCACAGCTCATC & TCTTGCCTCCAAAGTATGTCAA \\
\hline HO-1 & CTTCTTCACCTTCCCCAACA & GCTCTGGTCCTTGGTGTC \\
\hline y-GCS & GCACATCTACCACGCCGTC & CCACCTCATCGCCCCAC \\
\hline Catalase & GACTGACCAGGGCATCAAAAA & CGGATGCCATAGTCAGGATCTT \\
\hline IL-8 & GGACAAGAGCCAGGAAGAAA & AAATTTGGGGTGGAAAGGTT \\
\hline TNF $\alpha$ & TCAATCGGCCCGACTATCTC & CAGGGCAATGATCCCAAAGT \\
\hline TGF- $\beta 1$ & CCCTGGACACCAACTATTGC & CTTCCAGCCGAGGTCCTT \\
\hline COL1A1 & AAGAGGAAGGCCAAGTCGAG & CACACGTCTCGGTCATGGTA \\
\hline GAPDH & GAAGGCTGG GGCTCATTTG & AGGCTGTTGTCATACTTCTCATGG \\
\hline
\end{tabular}

\section{$2.11 \quad$ Nrf2 activation}

Nrf2 activation was measured in $10 \mu \mathrm{g}$ nuclear extracts using the TransAM Nrf2 activation kit (Active Motif, Carlsbad, CA) according to manufacturer's instructions. The extracts were prepared using a nuclear extraction kit (Active Motif).

\subsection{ROS production}

For the 2,7-dichlorofluorescein diacetate (DCF-DA) assay, cells were incubated with $10 \mu \mathrm{M}$ DCF-DA (Sigma) for $20 \mathrm{~min}$ at $37^{\circ} \mathrm{C}$, stimulated with bleomycin and fluorescence was measured with excitation at $485 \mathrm{~nm}$ and emission at $525 \mathrm{~nm}$.

\subsection{Statistical Analysis}

Quantitative data are presented as mean \pm SEMs. Two-sided differences between two groups were analyzed by unpaired t-tests. Differences among more than 2 groups were determined by 1-way ANOVA. Differences were considered significant if $P<0.05$. 


\section{Results}

\subsection{IPF patients have a decreased antioxidant capacity}

IPF is characterized by the presence of oxidative stress, which is defined as an imbalance between the production of ROS and protective against ROS by antioxidants, driving the pro-fibrotic response $(14,35)$. Although Nrf2 is upregulated in IPF to restore the pulmonary redox status, this protective response is not sufficient to protect against the elevated ROS level (18). Moreover, it is assumed that IPF patients have a dysregulated antioxidant response which is no longer able to counteract the increased ROS production. To evaluate the exact antioxidant status in our IPF patient group compared to healthy controls, the individual endogenous antioxidants $\mathrm{GSH}$, vitamin $\mathrm{C}$ and uric acid are determined as well as the total plasma antioxidant capacity (Figure 3.1).

The concentration of GSH is $50 \%$ decreased in the blood of IPF patients compared to the age-, gender- and dietary behavior matched controls (6.6 \pm 0.6 vs $12.9 \pm 1.9 \mu \mathrm{M} / \mathrm{mg} \mathrm{Hb}$ ). GSSG levels do not significantly differ between the two groups ( $0.8752 \pm 0.0926$ vs 0.6874 $\pm 0.0996 \mu \mathrm{M} / \mathrm{mg} \mathrm{Hb}$ ) (Figure 3.1A).

The trolox equivalent antioxidant capacity (TEAC value) is a measurement for the total antioxidant status, relating the free radical scavenging properties of a solution or a compound to that of the synthetic antioxidant trolox. The total plasma antioxidant capacity in the blood of IPF patients is significantly declined compared to that of matched controls (483.1 \pm 29.5 vs $619.7 \pm 16.6 \mu \mathrm{M}$ Trolox equivalent). The levels of the two endogenous antioxidants known to contribute substantially to the total plasma antioxidant status, i.e. uric acid ( $123.5 \pm 17.9$ vs $183.4 \pm 14.4 \mu \mathrm{M})$ and vitamin $\mathrm{C}(54.3 \pm 2.8$ vs $63.1 \pm 2.3 \mu \mathrm{M}$ ), are slightly decreased but vitamin $\mathrm{C}$ is not significantly changed in the plasma of IPF patients compared to controls (Figure 3.1B). 
A

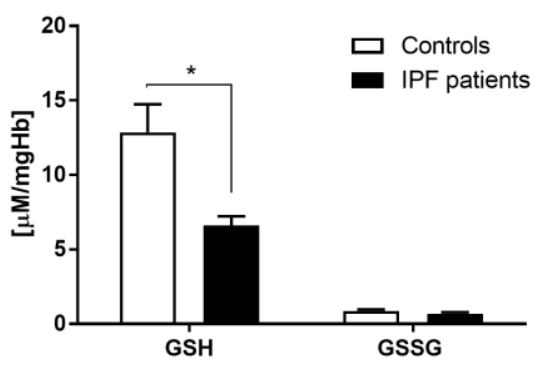

C

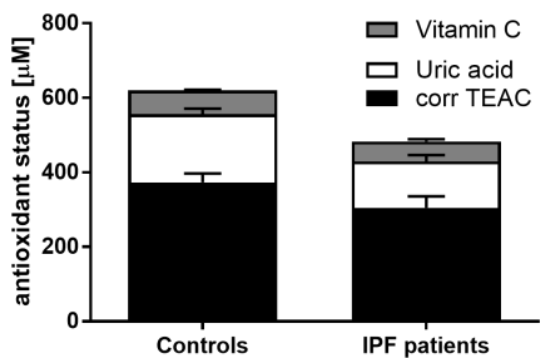

B

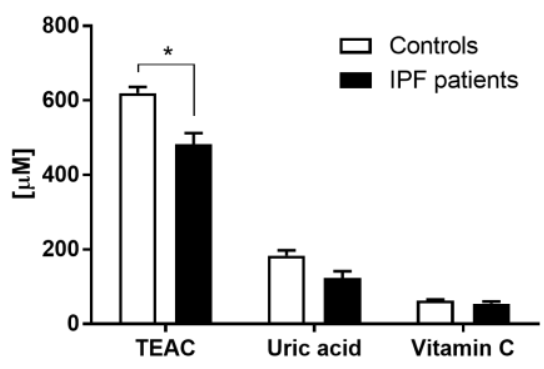

Figure 3.1: Idiopathic pulmonary fibrosis (IPF) patients have a decreased antioxidant capacity. Antioxidant status in IPF patients ( $n=11)$ compared to their matched controls $(n=9)$. (A) GSH ( $\mu M / m g ~ H b)$ and GSSG $(\mu M / m g ~ H b)$ measured in blood plasma. (B) Total antioxidant status (TEAC) $(\mu \mathrm{M})$, vitamin $C(\mu \mathrm{M})$ and uric acid $(\mu \mathrm{M})$, in blood plasma. (C) Relative plasma antioxidant capacity as well as contribution of uric acid $(\mu \mathrm{M})$ and vitamin $C(\mu \mathrm{M})$ to the total antioxidant status. Data are expressed as mean \pm SEM, ${ }^{*} \mathrm{P}<0.05,{ }^{* * P}<0.01$.

When the concentrations of uric acid and vitamin $\mathrm{C}$ are known, the relative contribution of these antioxidants to the total plasma antioxidant capacity can be calculated. This calculation shows that the major part of the total plasma antioxidant capacity is due to plasma antioxidants other than uric acid and vitamin $\mathrm{C}$, including low molecular protein thiols. This residual plasma antioxidant capacity (also referred to as corrected TEAC) is slightly but not significant lower in the fibrosis group compared to the matched controls (Figure 3.1C), indicating that IPF patients have a lower overall antioxidant capacity that is not solely due to decreased levels of its major players vitamin $\mathrm{C}$ and uric acid.

\subsection{Bleomycin increases in vitro ROS production which can be counteracted by quercetin}

We showed that IPF patients have a decreased antioxidant capacity and it is therefore expected that oxidative stress and a reduced antioxidant defense contribute to the development of the disease.

The effects of the pro-fibrotic trigger bleomycin on intracellular ROS production, as well as the possible protective effects of the strong antioxidant quercetin hereon, are evaluated 
in BEAS-2B cells. Cytotoxicity is not influenced by the different treatments as cell viability after stimulation with bleomycin and quercetin is more than $80 \%$ (data not shown).

Intracellular ROS production dose-dependently increases in response to bleomycin compared to the control (Figure 3.2A) with a 100\% increase upon stimulation with 30 $\mathrm{\mu g} / \mathrm{mL}$ bleomycin. Interestingly, pretreatment with various concentrations of the antioxidant quercetin leads to a concentration-dependent reduction of this bleomycininduced ROS production with the strongest effect (50\% reduction) occurring after pretreatment with $50 \mu \mathrm{M}$ quercetin (Figure 3.2B). However, for further experiments 25 $\mu \mathrm{M}$ quercetin is chosen as a higher dose $(50 \mu \mathrm{M})$ also induces a pro-inflammatory response in the cells (data not shown).
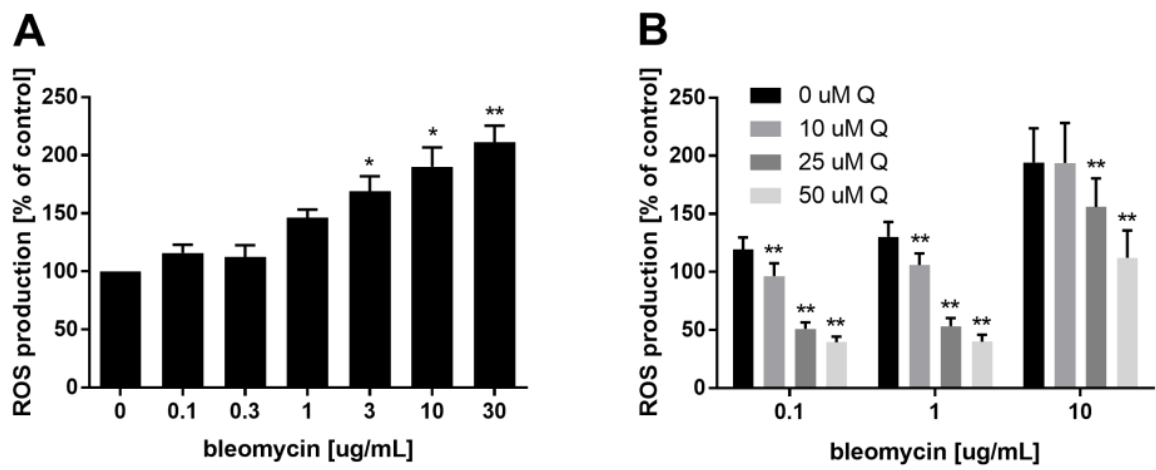

Figure 3.2: Bleomycin dose-dependently induces ROS production and pretreatment with quercetin can reduce this induced oxidant burden. ROS production by BEAS-2B cells after stimulation with different bleomycin concentrations for 2 hours using $10 \mu \mathrm{M}$ DCF-DA (A) without or (B) with quercetin pretreatment. Values are presented as percentage of fluorescence in the absence of bleomycin $\pm \operatorname{SEM}(n=6),{ }^{*} P<0.05,{ }^{* *} P<0.01$.

\subsection{Quercetin boosts antioxidant response by increasing Nrf2 activity and thereby also induction of Nrf2-regulated genes}

An increased ROS accumulation is known to induce the translocation of the redoxsensitive transcription factor Nrf2 to the nucleus, where it binds to the ARE causing gene transcription of antioxidant genes to compensate for oxidative stress in the cells (17). The effect of both the ROS-inducing bleomycin and the protective antioxidant quercetin on Nrf2 activity was analyzed. To this end, the gene expression of Nrf2 and Nrf2-regulated genes as well as the Nrf2 binding capacity are studied.

Bleomycin induces a slight, yet not significant increase in the expression of Nrf2, a significant $50 \%$ decrease in the expression of the Nrf2-regulated gene heme-oxygenase (HO-) 1, a not significant reduction in Nrf2-regulated $\nu$-glutamyl cysteine ( $\gamma$-GCS) expression and no change in the expression of Nrf2-regulated catalase (Figure 3A). Interestingly, pretreatment with quercetin strongly enhances BLM-induced expression of 
Nrf2 as well as of the Nrf2-regulated genes HO-1 and gamma-GCS, but not catalase, compared to treatment with bleomycin alone (Figure $3 \mathrm{~A}$ ). Next, the Nrf2 binding capacity to the ARE was evaluated. Bleomycin has no effect on Nrf2 activity whereas quercetin pretreatment causes a $30 \%$ significant increase in Nrf2 ARE binding compared to both the control and bleomycin-treated cells (Figure 3B).
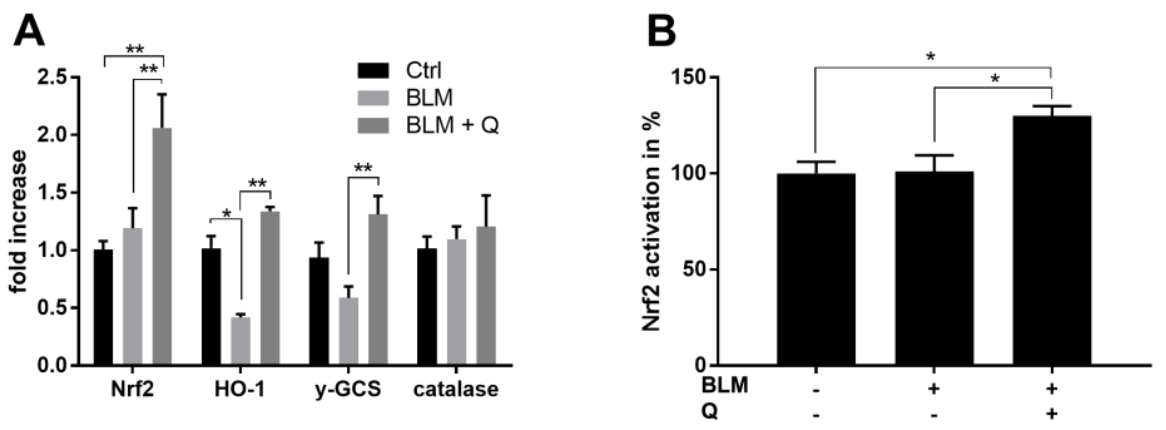

Figure 3.3: Quercetin increases Nrf2 activation. (A) Gene expression of Nrf2 and Nrf2-regulated genes after stimulation with $10 \mu \mathrm{g} / \mathrm{mL}$ bleomycin for 4 hours with or without pretreatment with $25 \mu \mathrm{M}$ quercetin for 30 min. Values are presented relative to GAPDH gene expression \pm SEM, (B) Nrf2 DNA binding after stimulation with 10 $\mu \mathrm{g} / \mathrm{mL}$ bleomycin for 4 hours with or without pretreatment with $25 \mu \mathrm{M}$ quercetin. Values are presented as percentage in the absence of bleomycin and quercetin \pm SEM $(n=4), * P<0.05, * * P<0.01$.

\subsection{Bleomycin dose-dependently induces IL-8 expression and production which can be reduced by quercetin pretreatment}

As previously described, inflammation plays an important role in the pathogenesis of IPF as the disease is characterized by the infiltration of inflammatory cells in the lungs (36). Additionally, it is known that ROS production and inflammation are closely intertwined. The next step was to investigate the effects of bleomycin and quercetin on both the gene expression and protein levels of the pro-inflammatory cytokines tumor necrosis factor- $\alpha$ (TNF $\alpha$ ) and interleukin-8 (IL-8) in vitro.

Bleomycin induces a 40-fold increase in IL-8 gene expression, whereas it does not affect TNF $\alpha$ gene expression (Figure 3.4A). Pretreatment with quercetin has no significant effect on this bleomycin-induced change in IL-8 gene expression.

Next, the effect of various bleomycin concentrations for $4 \mathrm{~h}$ and $24 \mathrm{~h}$ on the IL- 8 and TNF $\alpha$ production was investigated to see whether bleomycin has a short- or long-term effect on these cytokines. Bleomycin concentration-dependently increases IL-8 production after $4 \mathrm{~h}$ as well as after $24 \mathrm{~h}$ (Figure 3.4B and C). Pretreatment with $10 \mu \mathrm{M}$ quercetin has no effect on IL-8 production after $4 \mathrm{~h}$ whereas pretreatment with $25 \mu \mathrm{M}$ quercetin can significantly reduce IL-8 production induced by $10 \mu \mathrm{g} / \mathrm{mL}$ bleomycin (Figure 3.4D). After $24 \mathrm{~h}$ incubation with bleomycin, $25 \mu \mathrm{M}$ quercetin reduces this inflammatory response by $50 \%$ 
(Figure 3.4E). No increase in TNF $\alpha$ production can be measured upon bleomycin stimulation for either 4 or 24 hours (data not shown).

A

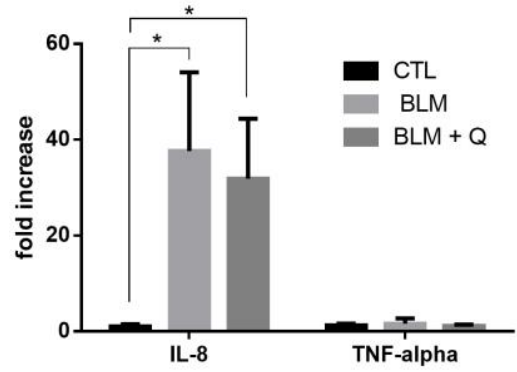

C

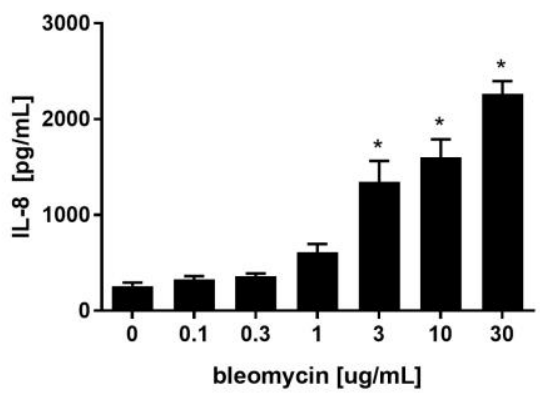

E

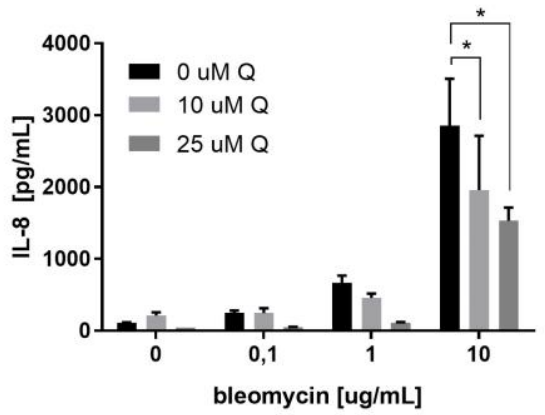

B

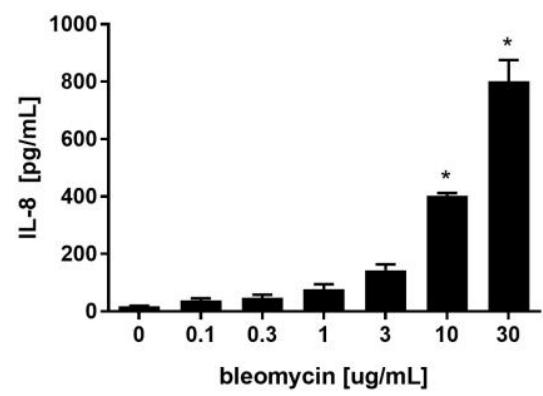

D

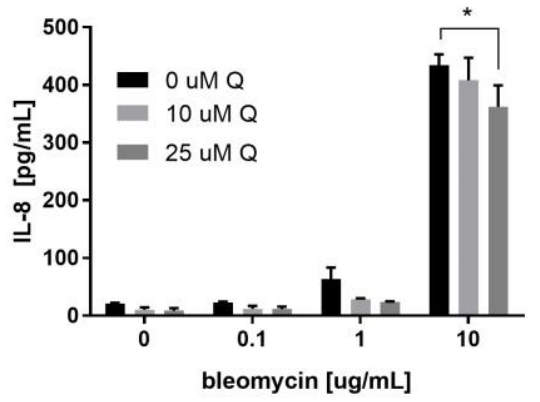

Figure 3.4: Bleomycin dose-dependently induces IL-8 gene expression and production which can be reduced by quercetin pretreatment. (A) Gene expression of pro-inflammatory genes after stimulation with $10 \mu \mathrm{g} / \mathrm{mL}$ BLM with or without pretreatment with $25 \mu \mathrm{M}$ quercetin. IL-8 production after stimulation with various concentrations of bleomycin after (B) $4 \mathrm{~h}$ and (C) $24 \mathrm{~h}$ of incubation. IL-8 production after pretreatment with different concentrations of quercetin and stimulation with bleomycin after (D) $4 \mathrm{~h}$ and (E) $24 \mathrm{~h}$ of incubation. Values are presented $\pm \operatorname{SEM}(n=6),{ }^{*} \mathrm{P}<0.05$. 


\subsection{IPF patients have a higher systemic inflammation which is reduced by quercetin ex vivo after LPS stimulation}

Our in vitro data suggest that the pro-inflammatory response after bleomycin challenge is mainly mediated by oxidative stress as the antioxidant quercetin is capable of protecting against this induced damage. To evaluate pro-inflammatory cytokines and the inhibiting effect of quercetin hereon in IPF patients, the inflammatory status and response are assessed in the plasma of IPF patients and matched controls.

Basal plasma levels of both IL-8 and TNF $\alpha$ (Figure 3.5A) show a significant increase in patients suffering from IPF compared to their controls $(13.1 \pm 2.1 \mathrm{pg} / \mathrm{ml}$ vs $7.8 \pm 0.4 \mathrm{pg} / \mathrm{ml}$ for IL-8 and $20.2 \pm 4.2 \mathrm{pg} / \mathrm{ml}$ vs $4.3 \pm 0.3 \mathrm{pg} / \mathrm{ml}$ for TNF $\alpha$ ).

To evaluate the anti-inflammatory effects of quercetin, inflammation is further evoked by ex vivo LPS-stimulation of the blood of both patients and controls. Ex vivo LPS-induced IL-8 and TNF $\alpha$ production are significantly decreased by quercetin in both the patient and the control group (Figure 3.5B and Figure 3.5C). Moreover, the extent of this reduction depends on the quercetin concentration. TNF $\alpha$ responds more sensitive to quercetin in the fibrosis group, resulting in a significant larger decrease in both TNF $\alpha$ production compared to the matched controls (Figure 3.5D). The same trend, although only significant for $30 \mu \mathrm{M}$ quercetin, is visible for LPS-induced IL-8 production. 
A

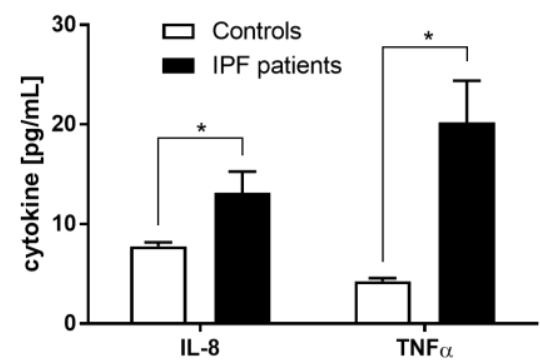

C

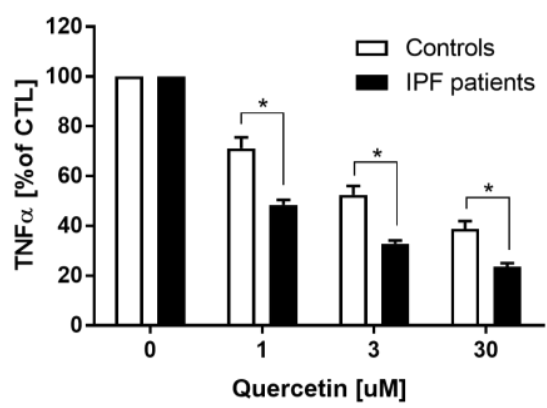

B

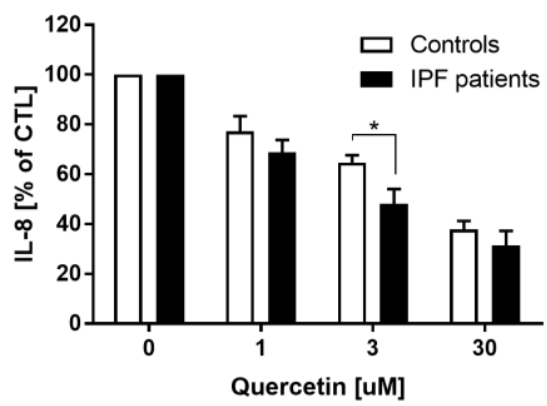

Figure 3.5: Idiopathic pulmonary fibrosis (IPF) patients have a higher basal inflammatory level which is dosedependently reduced by quercetin. (A) Basal cytokine levels in whole blood of IPF patients compared to their matched controls. (B) Ex vivo LPS-induced (24 hours) IL-8 production after 30 min preincubation with various concentrations of quercetin. (C) Ex vivo LPS-induced (24 hours) TNF $\alpha$ production after 30 min preincubation with various concentrations of quercetin. Cytokine production was related to that of the control incubation without quercetin $(100 \%)$. Values are represented as mean \pm SEM (controls $n=9$, IPF $n=11),{ }^{*} P<0.05,{ }^{* *} P<0.01$ 


\section{Discussion}

The pathogenesis of IPF, which is clinically characterized by excessive tissue scarring and an impaired wound healing response due to continuous insults of the alveolar epithelium, has been associated with an increased accumulation of ROS, resulting in a disturbed redox balance $(14,35)$. Recently, there has been a growing interest in the therapeutic use of strong natural antioxidants to prevent tissue damage induced by oxidative stress (21). Although some recent studies have explored the importance of oxidative stress in the development of IPF, the overall benefits of antioxidants to prevent and protect against IPF is largely unknown. The antioxidant quercetin, mainly present in fruit and vegetables, is not only known for its strong anti-oxidative but also for its anti-inflammatory capacities both in vitro and in various pulmonary models $(25,26)$. Additionally, studies indicate that it is also effective in restoring a disturbed redox balance and reducing inflammation in vivo in other interstitial lung diseases (ILD) such as sarcoidosis $(27,28)$. Moreover, quercetin also showed beneficial effects in cell or mouse models of a variety of lung diseases including lung cancer $(37), \operatorname{COPD}(38,39)$ and asthma $(40)$.

Based on these observations, we examined the potentially protective effects of the antioxidant quercetin, mainly present in fruit and vegetables, on markers of oxidative stress and inflammation in vitro and ex vivo in blood of IPF patients.

In summary, we show that IPF patients have a decreased antioxidant defense, indicated by a significantly reduced total antioxidant capacity, glutathione and uric acid concentrations in plasma compared to the controls. Our in vitro model displayed a dosedependent increase in ROS production and reduced expression of Nrf2-driven genes after challenge with the pro-fibrotic trigger bleomycin, indicating that the cells are not capable of dealing with the increased amount of oxidative stress. Moreover, basal TNF? and IL-8 levels are significantly increased in the plasma of IPF patients concurrent with our profibrotic in vitro model, in which IL-8 is dose-dependently increasing after bleomycin exposure. The dietary antioxidant quercetin boosts the antioxidant response in vitro, indicated by an increased expression of Nrf2 as well as Nrf2-regulated genes, including HO-1 and Y-GCS, and enhanced Nrf2 activity. The gene expression of catalase did not change after stimulation with bleomycin alone or in combination with quercetin. This can be explained by the facts that 1) catalase is not solely regulated by Nrf2, but also by other transcription factors including FoXO3a and PPARY (41) and 2) catalase upregulation is associated with $\mathrm{H}_{2} \mathrm{O}_{2}$-specific damage rather than the oxidative damage induced by bleomycin which mainly involves the production of $\mathrm{OH}^{-}$(42). Moreover, quercetin also significantly decreases the pro-inflammatory cytokine production in vitro as well as ex vivo in a concentration-dependent manner in both IPF patients and controls. Interestingly, this quercetin effect is more pronounced in IPF patients. Taken together, our ex vivo results show that IPF patients have a reduced antioxidant capacity and increased inflammatory 
status while our in vitro data demonstrate that quercetin might be a potential therapy option as it reduces markers of inflammation and oxidative stress, thereby also boosting the antioxidant response. The fact that the anti-inflammatory effects of quercetin are more pronounced in the patients than in their matched controls indicates that especially patients suffering from diseases associated with oxidative stress are expected to profit from the use of antioxidant supplementation. To our knowledge, this is the first study that not only showed the protective effects of quercetin in vitro but also ex vivo in blood of IPF patients.

In agreement with our results, previous studies have reported the antioxidant properties of quercetin in in vitro models of IPF. It has been shown that quercetin is a potent activator of HO-1 expression as well as Nrf2 activity, indicated by nuclear translocation, in human fibroblasts after stimulation with the pro-fibrotic trigger TGF- $\beta 1$ (43). Similar effects of quercetin have also been reported in a murine alveolar epithelial cell line (44). It is even suggested that quercetin is capable of inhibiting fibroblast activation, thereby ameliorating pro-fibrotic processes, indicated by collagen deposition in an experimental model of kidney fibrosis (45).

Besides lowering the occurrence of oxidative stress, reducing inflammation is also of great importance in IPF as pro-inflammatory cytokines can further evoke pro-oxidative and profibrotic processes in the lung. Quercetin has recently been suggested to reduce markers of inflammation in vitro and in vivo. It has been shown that quercetin reduces proinflammatory cytokines in models of acute and chronic inflammation (46) via the regulation of nuclear factor kappa-light-chain-enhancer of activated $B$ cells (NF-KB) and mitogen-activated protein kinases (MAPK) pathways, thereby directly suppressing the induction of pro-inflammatory cytokines $(47,48)$. Additionally, it has been shown before that ROS can induce the activation of several signaling pathways, including the redoxsensitive transcription factor NF-KB and MAPK, thereby inducing gene expression of various pro-inflammatory cytokines including TNF $\alpha, \operatorname{IL}-8$ and IL-1 $\beta(49,50)$. Consequently, it can be suggested that by reducing the amount of oxidative stress present in the cell, the release of pro-inflammatory cytokines can also be inhibited. This association between oxidative stress and inflammation can also be deducted from our results as the reduced antioxidant status of the IPF patients may explain the higher LPS-induced cytokine production in their blood and the more pronounced effects of quercetin hereon. Our results confirm this association as the antioxidant quercetin is not only lowering the oxidant burden but also reducing pro-inflammatory cytokine levels including IL-8 and TNF $\alpha$. Interestingly, after LPS stimulation to evoke acute inflammation, quercetin concentration-dependently reduces these pro-inflammatory cytokines in both controls and IPF patients, although the effect is more pronounced in IPF patients in general and in their TNF $\alpha$ response in specific. It is known that TNF $\alpha$ is highly expressed in the lungs of IPF patients and contributes to the diseases pathogenesis by initiating pro-inflammatory 
and pro-fibrotic pathways (51). Although inhibition of TNF $\alpha$ reduces bleomycin-induced fibrosis in a mouse model (52), treatment with a TNF $\alpha$ antagonist (Etanercept) in IPF patients did not show significant improvements on lung function (53). It might therefore be more beneficial to reduce TNF $\alpha$ production in an indirect manner by using quercetin rather than blocking the effects of TNF $\alpha$ completely.

Some limitations can be observed in the current study setup. Cells were stimulated with bleomycin, which is a commonly used model to induce pro-fibrotic responses in vitro as well as in vivo whereas the patient samples were stimulated with LPS to mimic an additional inflammatory burden. These two different triggers were selected since there was no need to induce a pro-fibrotic response in in vivo samples obtained from patients suffering from pulmonary fibrosis. In vivo, the ongoing inflammation in the IPF patients included in our study was mapped by analyzing the anti-inflammatory cytokines IL-8 and TNF- $\alpha$. IL-8, was selected due to its proven relation with IPF severity $(54,55)$ and the latter because anti-TNF- $\alpha$ agents have been suggested as treatment options for IPF. In vitro, the possible anti-inflammatory effects of quercetin during the onset of the disease were studied in an epithelial cell line that does not produce significant amounts of TNF- $\alpha$ but, since the involvement of IL- 8 has already been proven in vivo as well, the focus of the present study was on this cytokine rather than on TNF- $\alpha$.

To study the effect of quercetin, it is crucial that the study population has the same average intake of this dietary component. This average intake, which was approximately $15 \mathrm{mg}$ per day, was estimated using food questionnaires. Since the patient samples were stimulated with quercetin ex vivo, an additional clinical supplementation study needs to be performed to confirm our results. Safety, dose and tolerability associated with the long-term usage of quercetin still needs to be investigated. The safety of quercetin is of particular interest as it has been shown that quercetin can induce inflammation when given in higher doses. Additionally, quercetin can be converted into oxidation products which might interact with sulfhydryl groups, thereby impairing critical cellular functions (56). Moreover, assessing the absolute effect of quercetin in the current patient cohort might be hampered by the fact that four of the included IPF patients were taking the GSHprecursor NAC, one the corticosteroid prednisone and five a combination at the time of inclusion.

In the last 25 years, a multitude of compounds have been tested in clinical trials of IPF, but with almost invariably negative results (57). Such high rate of failure was probably due to both incomplete knowledge of disease pathogenesis and the multitude and redundancy of mediators, growth factors and signaling pathways involved in the fibrotic process (2).

Interestingly, the most promising results within IPF treatment are currently acquired using strong antioxidants, underlining the important role of ROS within the pathology of this disease. A meta-analysis evaluating the efficacy of antioxidant therapy in IPF recently 
described that antioxidant therapy combined with other therapies is more beneficial in regard to lung function (e.g. carbon monoxide diffusing capacity) compared to monotherapy without added antioxidants (58). However, anti-oxidant strategies with only NAC only have shown some potential benefits but cannot slow down the progression of the disease nor increase the overall survival (1). Possible explanation for this limited effect could be that the antioxidant profile of NAC does not fit $(1,14)$. Instead, other therapeutic strategies with another type of antioxidant, more potently enhancing the compensatory Nrf2 antioxidant response, may prove to be more effective (59). Especially for IPF treatment, this induction of Nrf2 is important since this redox-sensitive transcription factor coordinately induces a variety of self-defense genes, including antioxidant and phase II detoxification enzymes (60). Based on our results, a perfect candidate for such specific and directed antioxidant therapy would be the dietary antioxidant quercetin. Future research will include comparison of different antioxidant strategies to quercetin as an additional supplement for IPF treatment.

In conclusion, our preliminary data indicate that a reduced antioxidant defense, causing a disturbed redox balance, and inflammation are two key triggers in the pathophysiology of IPF. Moreover, we have shown that adding quercetin to the current available treatment options might be beneficial in maximizing treatment effects and slowing down disease progression. 


\section{References}

1. Woodcock HV, Maher TM. The treatment of idiopathic pulmonary fibrosis. F1000Prime Rep. 2014;6:16.

2. Raghu G, Collard HR, Egan JJ, Martinez FJ, Behr J, Brown KK, et al. An official ATS/ERS/JRS/ALAT statement: idiopathic pulmonary fibrosis: evidence-based guidelines for diagnosis and management. Am J Respir Crit Care Med. 2011;183(6):788-824.

3. Vancheri C, Failla M, Crimi N, Raghu G. Idiopathic pulmonary fibrosis: a disease with similarities and links to cancer biology. Eur Respir J. 2010;35(3):496-504.

4. Zoz DF, Lawson WE, Blackwell TS. Idiopathic pulmonary fibrosis: a disorder of epithelial cell dysfunction. The American journal of the medical sciences. 2011;341(6):435-8.

5. $\quad$ King TE, Jr., Pardo A, Selman M. Idiopathic pulmonary fibrosis. Lancet. 2011;378(9807):1949-61.

6. Raghu G, Chen SY, Yeh WS, Maroni B, Li Q, Lee YC, et al. Idiopathic pulmonary fibrosis in US Medicare beneficiaries aged 65 years and older: incidence, prevalence, and survival, 2001-11. Lancet Respir Med. 2014;2(7):566-72.

7. Meltzer EB, Noble PW. Idiopathic pulmonary fibrosis. Orphanet journal of rare diseases. 2008;3:8.

8. Navaratnam V, Fleming KM, West J, Smith CJ, Jenkins RG, Fogarty A, et al. The rising incidence of idiopathic pulmonary fibrosis in the U.K. Thorax. 2011;66(6):462-7.

9. Kuwano K, Nakashima N, Inoshima I, Hagimoto N, Fujita M, Yoshimi M, et al. Oxidative stress in lung epithelial cells from patients with idiopathic interstitial pneumonias. Eur Respir J. 2003;21(2):232-40.

10. Rahman T, Hosen I, Towhidul Islam MM, Shekhar HU. Oxidative stress and human health Adv Biosci Biotechnol. 2012;3:997-1019

11. Lenz AG, Costabel U, Maier KL. Oxidized BAL fluid proteins in patients with interstitial lung diseases. Eur Respir J. 1996;9(2):307-12.

12. Hecker L, Cheng J, Thannickal VJ. Targeting NOX enzymes in pulmonary fibrosis. Cell Mol Life Sci. 2012;69(14):2365-71.

13. Ushio-Fukai M, Nakamura Y. Reactive oxygen species and angiogenesis: NADPH oxidase as target for cancer therapy. Cancer Lett. 2008;266(1):37-52.

14. Mariken J.T.J Arts, J Sebastiaan Dallinga, Hans-Peter Voss, Guido R.M.M Haenen, Aalt Bast. A new approach to assess the total antioxidant capacity using the TEAC assay. Food Chem. 2004;88(4):567-70.

15. Cheresh P, Kim SJ, Tulasiram S, Kamp DW. Oxidative stress and pulmonary fibrosis. Biochim Biophys Acta. 2013;1832(7):1028-40.

16. Bast A, Haenen GR, Doelman CJ. Oxidants and antioxidants: state of the art. Am J Med. 1991;91(3C):2S-13S.

17. Cho HY, Reddy SP, Kleeberger SR. Nrf2 defends the lung from oxidative stress. Antioxidants \& redox signaling. 2006;8(1-2):76-87.

18. Singh S, Vrishni S, Singh BK, Rahman I, Kakkar P. Nrf2-ARE stress response mechanism: a control point in oxidative stress-mediated dysfunctions and chronic inflammatory diseases. Free radical research. 2010;44(11):1267-88.

19. Raghu G, Collard HR, Egan JJ, Martinez FJ, Behr J, Brown KK, et al. An official ATS/ERS/JRS/ALAT statement: idiopathic pulmonary fibrosis: evidence-based guidelines for diagnosis and management. Am J Respir Crit Care Med. 2011;183(6):788-824.

20. Raghu G, Rochwerg B, Zhang Y, Garcia CA, Azuma A, Behr J, et al. An Official ATS/ERS/JRS/ALAT Clinical Practice Guideline: Treatment of Idiopathic Pulmonary Fibrosis. An Update of the 2011 Clinical Practice Guideline. Am J Respir Crit Care Med. 2015;192(2):e3-19. 
21. Bast A, Weseler AR, Haenen GR, den Hartog GJ. Oxidative stress and antioxidants in interstitial lung disease. Curr Opin Pulm Med. 2010;16(5):516-20.

22. D'Andrea G. Quercetin: A flavonol with multifaceted therapeutic applications? Fitoterapia. 2015;106:256-71.

23. Numazawa S, Yoshida T. Nrf2-dependent gene expressions: a molecular toxicological aspect. The Journal of toxicological sciences. 2004;29(2):81-9.

24. Surh YJ, Kundu JK, Na HK. Nrf2 as a master redox switch in turning on the cellular signaling involved in the induction of cytoprotective genes by some chemopreventive phytochemicals. Planta medica. 2008;74(13):1526-39.

25. Boots AW, Wilms LC, Swennen EL, Kleinjans JC, Bast A, Haenen GR. In vitro and ex vivo anti-inflammatory activity of quercetin in healthy volunteers. Nutrition. 2008;24(7-8):703-10.

26. Gauliard B, Grieve D, Wilson R, Crozier A, Jenkins C, Mullen WD, et al. The effects of dietary phenolic compounds on cytokine and antioxidant production by $A 549$ cells. J Med Food. 2008;11(2):382-4.

27. Impellizzeri D, Talero E, Siracusa R, Alcaide A, Cordaro M, Maria Zubelia J, et al. Protective effect of polyphenols in an inflammatory process associated with experimental pulmonary fibrosis in mice. Br J Nutr. 2015;114(6):853-65.

28. Wang L, Chen J, Wang B, Wu D, Li H, Lu H, et al. Protective effect of quercetin on lipopolysaccharide-induced acute lung injury in mice by inhibiting inflammatory cell influx. Exp Biol Med (Maywood). 2014;239(12):1653-62.

29. Takashima K, Matsushima M, Hashimoto K, Nose H, Sato M, Hashimoto N, et al. Protective effects of intratracheally administered quercetin on lipopolysaccharide-induced acute lung injury. Respir Res. 2014;15:150.

30. Boots AW, Drent M, Swennen EL, Moonen HJ, Bast A, Haenen GR. Antioxidant status associated with inflammation in sarcoidosis: a potential role for antioxidants. Respir Med. 2009;103(3):364-72.

31. Boots AW, Drent M, de Boer VC, Bast A, Haenen GR. Quercetin reduces markers of oxidative stress and inflammation in sarcoidosis. Clin Nutr. 2011;30(4):506-12.

32. Quanjer PH, Tammeling, G.J., Cotes, J.E., Pedersom, O.F., Peslin, R., Yernaut., J.C. Lung volumes and forced ventilatory flows. Report working party. Standardization of lung functions tests. European Commission for Steel and Coal. Official statement of the European Respiratory Society. Eur Respir J. 1993;16(5-40).

33. Fischer MA, Gransier TJ, Beckers LM, Bekers O, Bast A, Haenen GR. Determination of the antioxidant capacity in blood. Clin Chem Lab Med. 2005;43(7):735-40.

34. Swennen EL, Bast A, Dagnelie PC. Immunoregulatory effects of adenosine 5'-triphosphate on cytokine release from stimulated whole blood. Eur J Immunol. 2005;35(3):852-8.

35. Kliment CR, Oury TD. Oxidative stress, extracellular matrix targets, and idiopathic pulmonary fibrosis. Free Radic Biol Med. 2010;49(5):707-17.

36. Bringardner BD, Baran CP, Eubank TD, Marsh CB. The role of inflammation in the pathogenesis of idiopathic pulmonary fibrosis. Antioxidants \& redox signaling. 2008;10(2):287-301.

37. Klimaszewska-Wisniewska A, Halas-Wisniewska M, Izdebska M, Gagat M, Grzanka A, Grzanka D. Antiproliferative and antimetastatic action of quercetin on A549 non-small cell lung cancer cells through its effect on the cytoskeleton. Acta Histochem. 2017;119(2):99-112.

38. Gunay E, Celik S, Sarinc-Ulasli S, Ozyurek A, Hazman O, Gunay S, et al. Comparison of the Anti-inflammatory Effects of Proanthocyanidin, Quercetin, and Damnacanthal on Benzo(a)pyrene Exposed A549 Alveolar Cell Line. Inflammation. 2016;39(2):744-51.

39. Warnakulasuriya SN, Ziaullah, Rupasinghe HP. Novel long chain fatty acid derivatives of quercetin-3-O-glucoside reduce cytotoxicity induced by cigarette smoke toxicants in human fetal lung fibroblasts. Eur J Pharmacol. 2016;781:128-38. 
40. Park HJ, Lee CM, Jung ID, Lee JS, Jeong YI, Chang JH, et al. Quercetin regulates Th1/Th2 balance in a murine model of asthma. Int Immunopharmacol. 2009;9(3):261-7.

41. Glorieux C, Zamocky M, Sandoval JM, Verrax J, Calderon PB. Regulation of catalase expression in healthy and cancerous cells. Free Radic Biol Med. 2015;87:84-97.

42. Hay J, Shahzeidi S, Laurent G. Mechanisms of bleomycin-induced lung damage. Arch Toxicol. 1991;65(2):81-94.

43. Nakamura T, Matsushima M, Hayashi Y, Shibasaki M, Imaizumi K, Hashimoto N, et al. Attenuation of transforming growth factor-beta-stimulated collagen production in fibroblasts by quercetin-induced heme oxygenase-1. Am J Respir Cell Mol Biol. 2011;44(5):614-20.

44. Hayashi $\mathrm{Y}$, Matsushima M, Nakamura T, Shibasaki M, Hashimoto N, Imaizumi K, et al. Quercetin protects against pulmonary oxidant stress via heme oxygenase-1 induction in lung epithelial cells. Biochem Biophys Res Commun. 2012;417(1):169-74.

45. Ren J, Li J, Liu X, Feng Y, Gui Y, Yang J, et al. Quercetin Inhibits Fibroblast Activation and Kidney Fibrosis Involving the Suppression of Mammalian Target of Rapamycin and beta-catenin Signaling. Sci Rep. 2016;6:23968.

46. Huang R, Zhong T, Wu H. Quercetin protects against lipopolysaccharide-induced acute lung injury in rats through suppression of inflammation and oxidative stress. Arch Med Sci. 2015;11(2):427-32.

47. Gardi C, Bauerova K, Stringa B, Kuncirova V, Slovak L, Ponist S, et al. Quercetin reduced inflammation and increased antioxidant defense in rat adjuvant arthritis. Arch Biochem Biophys. 2015;583:150-7.

48. Tao J, Wei Y, Hu T. Flavonoids of Polygonum hydropiper L. attenuates lipopolysaccharideinduced inflammatory injury via suppressing phosphorylation in MAPKs pathways. BMC Complement Altern Med. 2016;16:25.

49. Schuliga M. NF-kappaB Signaling in Chronic Inflammatory Airway Disease. Biomolecules. 2015;5(3):1266-83.

50. Mossman BT, Lounsbury KM, Reddy SP. Oxidants and signaling by mitogen-activated protein kinases in lung epithelium. Am J Respir Cell Mol Biol. 2006;34(6):666-9.

51. Rafii R, Juarez MM, Albertson TE, Chan AL. A review of current and novel therapies for idiopathic pulmonary fibrosis. J Thorac Dis. 2013;5(1):48-73.

52. Piguet PF, Collart MA, Grau GE, Kapanci Y, Vassalli P. Tumor necrosis factor/cachectin plays a key role in bleomycin-induced pneumopathy and fibrosis. J Exp Med. 1989;170(3):655-63.

53. Raghu G, Brown KK, Costabel U, Cottin V, du Bois RM, Lasky JA, et al. Treatment of idiopathic pulmonary fibrosis with etanercept: an exploratory, placebo-controlled trial. Am J Respir Crit Care Med. 2008;178(9):948-55.

54. Mukaida N. Pathophysiological roles of interleukin-8/CXCL8 in pulmonary diseases. Am J Physiol Lung Cell Mol Physiol. 2003;284(4):L566-77.

55. Ziegenhagen MW, Zabel P, Zissel G, Schlaak M, Muller-Quernheim J. Serum level of interleukin 8 is elevated in idiopathic pulmonary fibrosis and indicates disease activity. Am J Respir Crit Care Med. 1998;157(3 Pt 1):762-8.

56. de Franceschi L, Turrini F, Honczarenko M, Ayi K, Rivera A, Fleming MD, et al. In vivo reduction of erythrocyte oxidant stress in a murine model of beta-thalassemia. Haematologica. 2004;89(11):1287-98.

57. Spagnolo P, Del Giovane C, Luppi F, Cerri S, Balduzzi S, Walters EH, et al. Non-steroid agents for idiopathic pulmonary fibrosis. Cochrane Database Syst Rev. 2010(9):CD003134.

58. Kandhare AD, Mukherjee A, Ghosh P, Bodhankar SL. Efficacy of antioxidant in idiopathic pulmonary fibrosis: A systematic review and meta-analysis. Excli J. 2016;15:636-51.

59. Swamy SM, Rajasekaran NS, Thannickal VJ. Nuclear Factor-Erythroid-2-Related Factor 2 in Aging and Lung Fibrosis. American Journal of Pathology. 2016;186(7):1712-23. 
60. Singh S, Vrishni S, Singh BK, Rahman I, Kakkar P. Nrf2-ARE stress response mechanism: A control point in oxidative stress-mediated dysfunctions and chronic inflammatory diseases. Free Radical Res. 2010;44(11):1267-88. 



\section{Chapter 4}

\section{The dietary antioxidant quercetin reduces hallmarks of bleomycin-induced fibrogenesis in mice}

Carmen Veith*, Agnes W. Boots*, Catrin A. Albrecht, Roger Bartholome, Marie-José Drittij, Sandra M.H. Claessen, Aalt Bast, Martin Rosenbruch, Leonie Jonkers, Frederik-Jan van Schooten and Roel P.F. Schins.

*equal contribution

Submitted 


\section{Abstract}

\section{Background}

Interstitial pulmonary fibrosis (IPF) is a chronic, lethal disease of which the etiology is still not fully understood. Current treatment comprises two FDA-approved drugs that can slow down yet not stop or reverse the disease. As IPF pathology is associated with an altered redox balance, redox modulating components might exert beneficial effects. Quercetin is a dietary antioxidant with strong redox modulating capacities that is suggested to exert part of its antioxidative effects via activation of the redox-sensitive transcription factor Nrf2 that regulates endogenous antioxidant levels. Therefore, the aim of the present study was to investigate if the dietary antioxidant quercetin can exert anti-fibrotic effects in a mouse model of bleomycin-induced pulmonary fibrogenesis through Nrf2-dependent restoration of the redox imbalance.

\section{Methods}

Homozygous Nrf2 deficient mice and their wildtype littermates were fed a control diet without or with $800 \mathrm{mg} / \mathrm{kg}$ quercetin from 7 days prior to a single $1 \mu \mathrm{g} / 2 \mu \mathrm{l}$ per g BW bleomycin challenge until they were sacrificed 14 days afterwards. Lung tissue and plasma were collected to determine markers of fibrosis, inflammation and redox balance.

\section{Results}

Mice fed the quercetin enriched diet for 7 days prior to the bleomycin challenge had significantly enhanced plasma quercetin levels $(11.08 \pm 0.73 \mu \mathrm{M}$ versus $7.05 \pm 0.2 \mu \mathrm{M})$ combined with increased expression of Nrf2 and Nrf2-responsive genes compared to mice fed the control diet in lung tissue. Upon bleomycin treatment, quercetin-fed mice displayed reduced expression of collagen (COL1A2) and fibronectin (FN1) and a tendency of reduced inflammatory lesions $(2.8 \pm 0.7$ versus $1.9 \pm 0.8)$. These beneficial effects were accompanied by reduced pulmonary gene expression of TNF $\alpha$ and KC, but not their plasma levels, as well as enhanced Nrf2-induced pulmonary antioxidant defences. In Nrf2/animals, no effect of the dietary antioxidant on either histology or inflammatory lesions was observed.

\section{Conclusion}

Quercetin exerts anti-fibrotic and anti-inflammatory effects on bleomycin-induced pulmonary damage in mice possibly through modulation of the redox balance by inducing Nrf2. However, quercetin could not rescue the bleomycin-induced pulmonary damage indicating that quercetin alone cannot ameliorate the progression of IPF.

KEYWORDS: bleomycin, dietary supplementation, inflammation, mice, oxidative stress 


\section{Introduction}

IPF is a progressive and often lethal disease with a European prevalence of 23 cases and incidence of up to 7 cases per 100.000 and a median survival of 3-5 years after diagnosis $(1,2)$. IPF prevalence and incidence, both positively correlated with age, have increased during the recent years, presumably due to the rapid expansion of the elderly population $(1,3)$. Key clinical feature of IPF is a cruelly impaired lung function, characterized by progressive dyspnea (difficulty breathing) and a non-productive cough, resulting in a drastically reduced vital capacity and thus quality of life (4). Current IPF treatment involves two newly FDA-approved drugs Pirfenidone ${ }^{\odot}$ and Nintedanib ${ }^{\odot}$ that are capable of slowing down the progression of the disease but cannot stop nor cure the disease (5-8). Additionally, they are not equally effective in all patients and it is still not clear which patient would benefit best from what drug. Consequently, there is an urgent need for additional effective therapy options (6). However, in order to meet this necessity, more knowledge regarding the exact pathogenesis of IPF is compulsory.

The pathogenesis of IPF is a combination of genetic predisposition and common environmental triggers, including bacteria and cigarette smoke $(2,9)$, leading to multiple and continuous insults to the lung epithelium. Such insults will cause early onset inflammation shading off into impaired wound healing and excessive tissue scarring. Main characteristic of such insults is a disturbed redox balance $(10,11)$, i.e. an overload of reactive oxygen species (ROS) in comparison to the presence of protective antioxidants. An increased oxidant burden will cause severe oxidative damage and trigger up-regulation of a number of genes involved in inflammation and/or fibrogenesis (11). Besides being a pathological feature of IPF, as can be deducted from the higher levels of various oxidative damage markers in these patients including exhaled ethane, nitric oxide, malondialdehyde levels and elevated urinary isoprostane levels (12-15), oxidants are also an important player in the development of the disease (16). Indeed, oxidative stress mediates several epithelial and fibroblastic alterations that influence lung homeostasis and hamper pulmonary repair and regeneration pathways. Interestingly, these oxidative changes will be further enhanced by the age-dependent increased oxidant production associated with IPF, thereby contributing to pro-fibrotic conditions $(11,16)$.

To offer protection against oxidant-induced damage, our body comprises an elaborate antioxidant defense system that is largely controlled by antioxidant genes $(13,17)$. Upon activation by oxidants, the redox-sensitive transcription factor Nrf2 induces the transcription of antioxidants as a feedback mechanism to restore systemic redox balance and maintain homeostasis (18). Indeed, exposure to oxidative stimuli such as cigarette smoke and particulate matter up-regulates Nrf2-dependent antioxidant genes in mice carrying the functional transcription factor (19-21). Moreover, the loss of Nrf2 results in the development of accelerated, more severe lung emphysema, oxidative stress, epithelial 
damage and enhanced sensitivity towards oxidants after exposure to oxidative stimuli (18, $22,23)$. In IPF, an increased pulmonary Nrf2 expression as well as elevated antioxidant levels in the broncho-alveolar fluid are reported (24), suggesting the existence of a compensatory, Nrf2-driven feedback mechanism that does increase the pulmonary antioxidant levels but not to the extent that they can completely restore systemic redox balance and maintain homeostasis in IPF.

Due to the large involvement of ROS in the pathophysiology of IPF, some antioxidants including $\mathrm{N}$-acetylcysteine (NAC) (25) and pirfenidone (5) have already been implemented as therapy in order to reduce the increased oxidative burden in IPF. However, although NAC supplementation reduced collagen deposition in bleomycin exposed rats (26) purely oxidant scavenging has not been enough to completely cure or prevent IPF until now (8). Consequently, there is an urgent need for an additional therapeutic approach that will exert health beneficial effects by modulating the important oxidant-related pro- and antifibrotic pathways rather than by solely scavenging antioxidants. A good candidate for such an approach would be a non-pharmacological compound with a known working mechanism that compiles of more than purely scavenging capacities. The dietary antioxidant and redox modulator quercetin could qualify as such a suitable alternative.

Quercetin is a member of the flavonoids family, dietary antioxidants that are ubiquitously present in vegetables, fruit, tea and wine (17). The daily Dutch intake has been estimated at several hundreds of $\mathrm{mg} / \mathrm{day}$ (27) of which the subfamily flavones and flavonols to which quercetin belongs delivers up to $16 \mathrm{mg} /$ day (27). The most abundant flavonol is quercetin, representing $70 \%$ of this intake, which has been shown to act as a powerful phenolic antioxidant both in vitro and in vivo $(17,27)$. By scavenging oxidants, quercetin can offer direct protection against both oxidative damage and inflammation, two key processes in the development of lung fibrosis. Indeed, we have already demonstrated that quercetin reduces markers of oxidative stress and inflammation in IPF and sarcoidosis $(28,29)$.

Additionally, quercetin may indirectly improve the antioxidant defense by increasing Nrf2driven antioxidant production. Oxidants activate Nrf2 by oxidizing its inhibitory protein Keap1, after which Nrf2 is released and translocated to the nucleus $(19,30)$. In the nucleus, Nrf2 binds to the antioxidant response element (ARE) and thus activates various antioxidant-genes including superoxide dismutase, glutathione peroxidase and heme oxygenase $1(28,30)$. Interestingly, Nrf2 is suggested to be a specific target for phenolic antioxidants (30) presumably via its release from Keap1 upon thiol arylation (31). In that way, quercetin will not only protect against a-specific damage caused by increased oxidant levels but will also further increase specific protection against these oxidants.

The aim of our study was to investigate if the dietary antioxidant quercetin can exert antifibrotic effects in a mouse model of bleomycin-induced pulmonary fibrogenesis through Nrf2-dependent restoration of redox imbalance. 


\section{Material and Methods}

\subsection{Mouse model}

Experiments were performed using homozygous Nrf2 deficient ( $\mathrm{Nrf2}^{-/}$) mice (RIKEN, Japan) that were backcrossed to C57BL/6J at least 9 times (32). The homozygous mutant $\left(\mathrm{Nrf2}^{-/}\right)$and wildtype $\left(\mathrm{Nrf2}^{+/+}\right)$littermates from both genders, produced from heterozygous breeding, were used in the present study at an age of 10-12 weeks. A pilot kinetic study was performed in C57BL/6J mice. Animals were kept in an in-house pathogen-free and environmentally controlled facility (safety level 1) according to guidelines of the Society for Laboratory Animals Science (GVSOLAS). A 12 hours light/dark cycle was maintained and food and water provided ad libitum. Ethical approval was given by the North Rhine Westphalia State Agency for Nature, Environment and Consumer Protection (NRW-LANUV).

\subsection{Quercetin diet and evaluation of effectiveness}

Animals had ad libitum access to either the control diet (AIN893, ssniff Spezialdiäten, Soest Germany) or the control diet enriched with $800 \mathrm{mg} / \mathrm{kg}$ quercetin (Merck, Darmstadt, Germany) starting from 7 days prior to the bleomycin challenge until they were sacrificed. Quercetin was stabilized (I) by adding 0.1 gram citric acid per liter water while preparing both diets and (II) by replacing food pellets by a freshly thawed stock on a daily basis while providing both diets. Based on a daily intake of 5 grams food per day and an average weight of 20 grams, this quercetin-enriched diet will result in an average daily intake of $200 \mathrm{mg}$ quercetin/kilogram BW. Weight gain, general signs of (dis)comfort and illness as well as survival were recorded on a daily basis throughout the entire dietary supplementation to ensure the safety of this intervention. To evaluate its effectiveness, six C57BL/6J mice were fed for one week with quercetin enriched or control diet and sacrificed to determine quercetin concentrations in the plasma, lung and in the gastrointestinal (GI) tract and as well the expression of Nrf2 and Nrf2-responsive antioxidant genes.

\subsection{Intervention study}

After 1 week of quercetin-enriched or control diet, $\mathrm{Nrf2}^{-/-}$and $\mathrm{Nrf2}^{+/+}$mice were exposed to $1 \mu \mathrm{g} / 2 \mu \mathrm{l}$ per gram BW bleomycin in $0.9 \% \mathrm{NaCl}$ or $0.9 \% \mathrm{NaCl}$ by a single pharyngeal aspiration under isoflurane anaesthesia. After 14 days, animals were brought under deep anaesthesia using $50 \mathrm{mg} / \mathrm{kg}$ body weight pentobarbital and euthanized via exsanguination after which blood and lungs were collected by respectively cardiac puncture and surgical removal. Left lungs were used for histology and processed as described below. The lobes of the right lungs were equally divided in 2 parts, one for RNA extraction and one for homogenate preparation, and snap-frozen until further analysis. Blood was centrifuged $\left(10^{\prime}, 1200 \mathrm{~g}, 4^{\circ} \mathrm{C}\right)$ after which plasma aliquots were also snap-frozen until further analysis. 
A schematic overview of the experimental setup, including the number of animals per group, is provided in Figure 4.1. For all groups, littermates were used to ensure optimal comparison of the influence of both factors on the primary and secondary endpoints of this study.

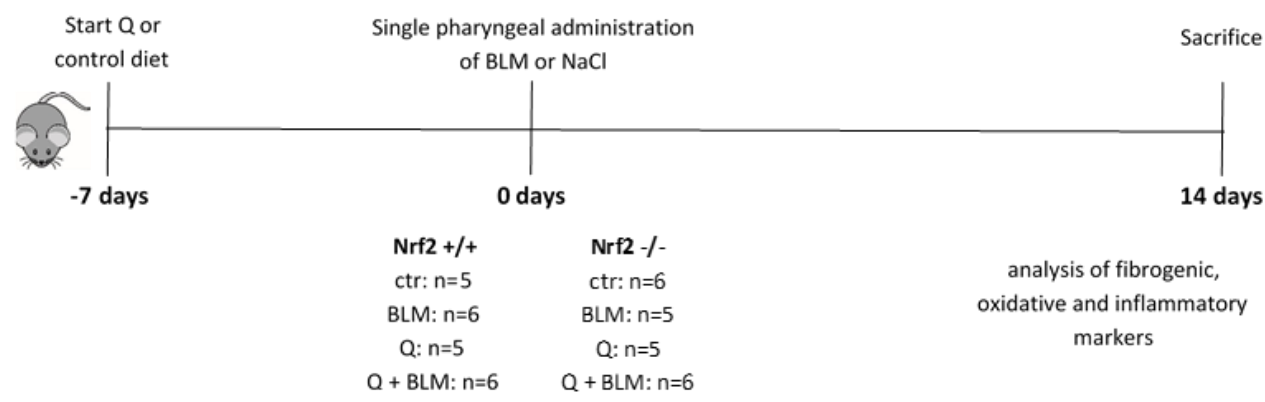

Figure 4.1: Schematic overview of the study design ( $Q=$ quercetin; $B L M=$ bleomycin).

\subsection{Histopathological evaluation of lung tissues}

Lungs were fixed in $4 \%$ paraformaldehyde /PBS, pH 7.4, dehydrated in a series of ethanol and subsequently xylol and embedded in paraffin. Sections of the left lungs were cut at a thickness of $4 \mu \mathrm{m}$, rehydrated and stained with H\&E (hematoxylin and eosin) and Masson's Trichrome. Subsequently, slides were evaluated histopathologically applying a semiquantitative grading: 1 =minimal, 2 =slight, 3 =marked, $4=$ =severe, $5=$ =massive.

\subsection{RNA isolation}

To isolate RNA from mouse lungs, the tissue was first homogenized using a tissue homogenizer in $700 \mu \mathrm{L}$ Qiazol (Invitrogen, Carlsbad, CA). Afterwards, the samples were incubated for $5 \mathrm{~min}$ at room temperature, $150 \mu \mathrm{L}$ chloroform were added and the solutions were mixed. The samples were centrifuged for $15 \mathrm{~min}$ at $12.000 \mathrm{rpm}$ and the upper aqueous layer was used for RNA isolation.

RNA was isolated and purified using the RNeasy mini kit (Qiagen, Venlo, the Netherlands) according to the manufacturer's instructions. The RNA concentration was determined using a Nanodrop spectrophotometer (Thermo Scientific, Waltham, MA).

\subsection{Real-time PCR}

cDNA was synthesized from 300 ng isolated RNA using IScript (Biorad, Hercules, CA) according to the manufacturer's instructions. RT-PCR was performed using SYBR Green PCR Supermix (BioRad) with $2.5 \mu \mathrm{L}$ of 10 times diluted CDNA and $0.4 \mu \mathrm{mol} / \mathrm{L}$ predesigned primers and PCR amplifications were carried at $95^{\circ} \mathrm{C}$ for $10 \mathrm{sec}$ for denaturation and 40 cycles of annealing/elongation $\left(60^{\circ} \mathrm{C}, 30 \mathrm{sec}\right)$ for selected genes (Table 1). The gene expression was normalized to the house keeping gene $\beta$-actin and quantified according to the $2^{-\Delta \Delta c t}$ method to relatively quantify the expression of the genes of our interest. The influence of quercetin on the redox-effects of bleomycin was analyzed by measuring the 
expression of Nrf2 and the Nrf2-responsive genes HO-1, y-GCS, SOD2, and CAT. The alleged anti-inflammatory effects of the dietary intervention were investigated by including CXCL-1 (KC) and TNF- $\alpha$, whereas COL1A2 and FN1, were added as pro-fibrotic genes of interest.

Table 4.1: Mouse RT-PCR forward and reverse primer sequences

\begin{tabular}{|l|l|l|}
\hline Gene of interest & Forward primer sequence & Reverse primer sequence \\
\hline$\beta$-actin & CTGAATGGCCCAGGTCTGA & CCCTCCCAGGGAGACCAA \\
\hline NRF2 & GCAGGCTATCTCCTAGTTCTCC & GCTACTTGCAGCAGAGGTGA \\
\hline HO-1 & GAGCCTGAATCGAGCAGAAC & CCTTCAAGGCCTCAGACAAA \\
\hline y-GCS & TGCAGGAGCAGATTGACAGG & TAGAGAAAGCAAGCGGGTGG \\
\hline SOD2 & GGCCAAGGGAGATGTTACAA & ACCCTTAGGGCTCAGGTTTG \\
\hline CAT & AGCGACCAGATGAAGCAGTG & TCCGCTCTCTGTCAAAGTGTG \\
\hline CXCL1 (KC) & GGTGAGGACATGTGTGGGAG & CGAGACCAGGAGAAACAGGG \\
\hline TNF- $\alpha$ & CAGCGCTGAGGTCAATCTGCC & TGCCCGGACTCCGCAA \\
\hline COL1A2 & GCAGGTTCACCTACTCTGTCCT & CTTGCCCCATTCATTTGTCT \\
\hline FN1 & CCCTGTTCTGCTTCAGGGTT & AAAGCAGAGGTGTCTGGGTG \\
\hline
\end{tabular}

\subsection{Homogenate preparation}

From the collected right lung tissue, homogenates were made by crushing with liquid nitrogen. Before crushing, the weight of the tissue was determined. Afterwards, 3 parts of sodium phosphate buffer $(145 \mathrm{mM}, \mathrm{pH}=7.4)$ was added to the crushed tissue and protein content was measured using Pierce BCA protein assay kit according to manufacturer instructions.

\subsection{Determination of total plasma quercetin concentration}

Total plasma quercetin concentration was measured as previously described (33) after enzymatic hydrolysis using high performance liquid chromatography (HPLC) with colorimetric array-detection.

\subsection{Luminex (Bio-Plex cytokine assay)}

Plasma cytokine profiles were determined with Bio-Plex assays (Bio-Rad) using Luminex XMAP-technology. To quantify the concentrations of 6 different cytokines, we used a BioPlex murine cytokine 11-plex panel including IL-4, TNF $\alpha$, KC, MIP2, MCP-1, IL-13, IL-1 3 , IL17a, IL-10, MIP1- $\alpha$ and MIP-1 $\beta$. All assays were performed as described by the manufacturer's instructions. Briefly, $50 \mu \mathrm{L}$ of the antibody magnetic beads were added to each well and unbound antibody was removed. Next, $25 \mu \mathrm{L}$ plasma were added to $25 \mu \mathrm{L}$ universal assay buffer and incubated for $2 \mathrm{~h}$. The plate was washed and $25 \mu \mathrm{L}$ detection antibody, which binds to its corresponding analyte present in the sample, were added for $30 \mathrm{~min} .50 \mu \mathrm{L}$ Streptavidin-PE were added after washing and incubated for $30 \mathrm{~min}$. After 
washing, the beads were resuspended in $120 \mu \mathrm{L}$ reading buffer. Next, the bead and reporter quantity of the antibody sandwich formed around the analyte was determined by a laser detector. Finally, the beads and reporter quantities measured were compared to those of an internal standard corresponding to each specific cytokine. Data analysis was done with a Luminex 100 IS 2.3 system using the Bio-Plex Manager 4.1.1 software.

\subsection{Trolox antioxidant capacity}

The trolox equivalent antioxidant capacity (TEAC value) is a measurement for the total antioxidant status, assessing the capacity of a compound to scavenge ABTS radicals. This assay has been performed as described earlier (34) with some minor modifications. In short, blood was centrifuged (3000 rpm, $5^{\prime}$ at 4 으) and plasma was afterwards deproteinized, using 10\% TCA (1:1) followed by centrifugation (13.000 rpm, $5^{\prime}$ at 4 으). A solution of $0.23 \mathrm{mM}$ ABTS (2,2'-azinobis-(3-ethylbenzothiazoline-6-sulfonic acid)) and $2.3 \mathrm{mM}$ ABAP $\left(2,2^{\prime}\right.$-Azobis-(2-amidinopropane) $\left.\mathrm{HCl}\right)$ was incubated at $70^{\circ} \mathrm{C}$ to generate $A B T S$ radicals $\left(A B T S^{\circ}\right)$ until the absorbance at $734 \mathrm{~nm}$ reached $0.70 \pm 0.02$. To measure the antioxidant capacity of the samples, $50 \mu \mathrm{l}$ of the plasma sample was mixed with $950 \mu \mathrm{l}$ ABTS solution and incubated for $5 \mathrm{~min}$ at $37^{\circ} \mathrm{C}$. The decrease in absorbance was measured at $734 \mathrm{~nm}$ and related to the absorbance of trolox standards to calculate the trolox equivalent of the samples.

\subsection{Malondialdehyde-dG DNA adducts ( $\left.\mathbf{M}_{1}-\mathrm{dG}\right)$}

The presence of $\mathrm{M}_{1}-\mathrm{dG}$ as was evaluated using 32P-postlabeling as previously described (Peluso et al, 2013). To this end, DNA was first hydrolysed by incubating in $2.5 \mathrm{mM}$ calcium chloride for 4.5 hours at $37^{\circ} \mathrm{C}(\mathrm{pH} 6.0)$ in the presence of $21.45 \mathrm{mU} / \mu \mathrm{l}$ micrococcal nuclease and $6.0 \mathrm{mU} / \mu \mathrm{l}$ spleen phosphodiesterase in $5.0 \mathrm{mM}$ sodium succinate. Upon hydrolysis, samples were incubated with $0.1 \mathrm{U} / \mu \mathrm{lNP1}$ in $46.6 \mathrm{mM}$ sodium acetate $(\mathrm{pH} 5.0)$ and $0.24 \mathrm{mM} \mathrm{ZnCl} 2$ for 30 minutes at $37^{\circ} \mathrm{C}$. Next step after this NP1 treatment was the generation of ${ }^{32} \mathrm{P}$-labelled DNA adducts in bicine buffer $(20 \mathrm{mM}$ bicine, $10 \mathrm{mM} \mathrm{MgCl}, 10$ $\mathrm{mM}$ dithiotreithol, $0.5 \mathrm{mM}$ spermidine, $\mathrm{pH} 9.0$ ) by adding $1.8 \mu \mathrm{l}$ of $0.16 \mathrm{mM}$ Tris base and incubating at $37^{\circ} \mathrm{C}$ for 30 minutes with $7-25 \mu \mathrm{Ci}$ of carrier-free [y-32 P]ATP (3000 Ci/mM) and polynucleotide kinase $\mathrm{T} 4(0.75 \mathrm{U} / \mu \mathrm{l})$. These generated ${ }^{32} \mathrm{P}$-labeled samples were then applied to polyethyleneimine cellulose thin layer chromatography plates (MachereyNagel, Germany) and resolved in a low-urea solvent system as previously described (35). Storage phosphor imaging techniques employing intensifying screens from Molecular Dynamics (Sunnyvale, California) were used to detect and quantify $\mathrm{M}_{1}-\mathrm{dG}$ adducts as well as normal nucleotides, i.e. diluted untreated samples. Levels of $M_{1}$-dG adducts were expressed as relative adduct labelling, i.e. as pixels in adducted nucleotides/pixels in normal nucleotides, and corrected across experiments based on the recovery of an MDAtreated DNA adduct standard. 


\subsection{Statistics}

All quantitative data are represented as means \pm SEM. Statistical differences between groups were evaluated by the nonparametric Mann-Whitney U-test using GraphPad Prism software (version 7.3; GraphPad Software, La Jolla, CA), and considered significant at a $P$ value of less than 0.05 . 


\section{Results}

\subsection{Effectiveness of quercetin supplementation}

Prior to applying the bleomycin challenge, the safety and effectiveness of the quercetin supplementation were assessed by analysing food intake as well as the body weight and overall survival of $\mathrm{C} 57 \mathrm{BI} / 6 \mathrm{~J}$ mice from our in house breeding colony fed either the control or quercetin-enriched diet. As can be deducted from Figure 4.2A, quercetin concentrations in the diet were stable throughout the study in both the frozen and room temperature pellets. Average body weight gain (Figure 4.2B) was not affected by diet nor were food intake and survival (data not shown). This 1-week supplementation resulted in significantly higher plasma quercetin levels compared to those levels in animals fed the control diet $(11.08 \pm 0.73 \mu \mathrm{M}$ versus $7.05 \pm 0.2 \mu \mathrm{M}$; Figure $4.2 \mathrm{C}, \mathrm{p}<0.01)$. However, quercetin supplementation did not significantly increase quercetin concentrations in the lungs $(2.97 \pm 0.21 \mathrm{nmol} / \mathrm{g}$ versus $1.95 \pm 1.06 \mathrm{nmol} / \mathrm{g})$ and $\mathrm{Gl}$ tract $(5.05 \pm 1.9 \mathrm{nmol} / \mathrm{g}$ versus $2.89 \pm 0.35 \mathrm{nmol} / \mathrm{g}$ ). Additionally, dietary supplementation of quercetin for 1 week increased the pulmonary expression levels of Nrf2 and the Nrf2-responsive genes $\mathrm{Y}$-GCS, HO- 1 and CAT of which the latter was significantly enhanced by the quercetin diet (figure 4.2D, $p<0.05)$.

A

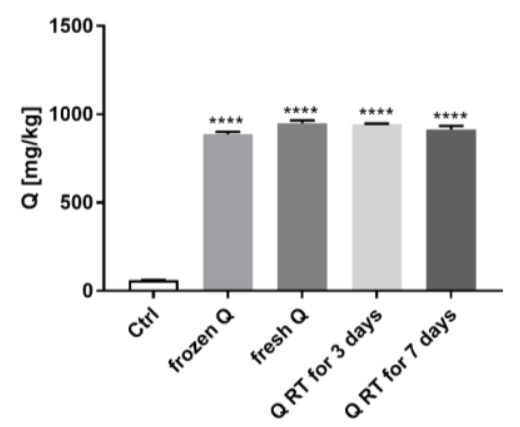

C

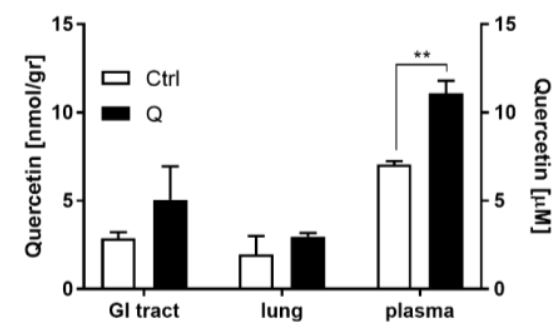

B

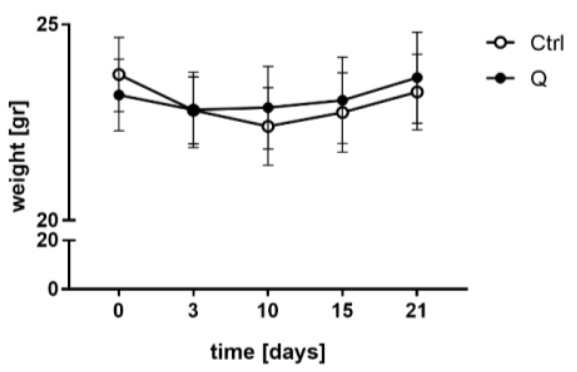

D

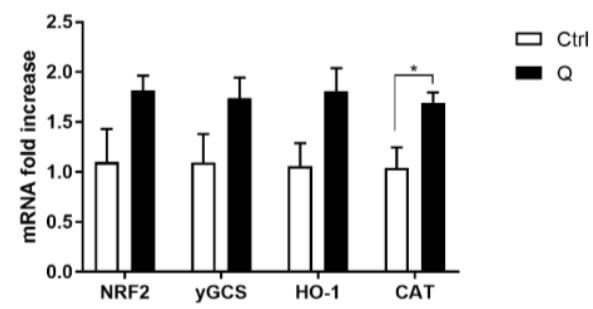

Figure 4.2: One-week dietary quercetin supplementation safely enhances plasma and pulmonary quercetin levels as well as pulmonary expression of Nrf2 and Nrf2-responsive genes. (A) quercetin concentration in differently stored diet aliquots, (B) weight gain in animals fed either the control $(n=3)$ or quercetin-enriched $(n=3)$ diet for 1 week, (C) quercetin concentrations in different organs after 1 week, (D) expression of Nrf2 and Nrf2-responsive 
genes after 1-week dietary intervention. Data are expressed as mean $\pm \mathrm{SEM} ;{ }^{*}=\mathrm{P}<0.05 ; * *=\mathrm{P}<0.01(\mathrm{n}=3$ per treatment)

\subsection{Quercetin reduces BLM-induced pulmonary fibrogenesis}

Upon verifying the pulmonary and systemic uptake of quercetin when supplemented via the diet over a period of 1 week in an independent pilot study, $\mathrm{Nrf2}^{+/+}$mice received a single bleomycin challenge via pharyngeal aspiration to induce pulmonary fibrosis. In this model, fibrosis has been found to develop from 7-10 days and peaks at 14-21 days after bleomycin injury (36). Two weeks after the bleomycin challenge, histological examination of H\&E as well as Masson's Trichrome-stained sections of the lungs revealed a thickening of the airway epithelium in several bleomycin-treated $\mathrm{Nrf2}^{+/+}$mice. Additionally, all lungs of bleomycin-treated $\mathrm{Nrf}^{+/+}$mice revealed multi-focal inflammatory lesions, predominantly in the more central region around the main airways. These inflammatory lesions mainly consisted of mononuclear cells, fibroblasts and alveolar macrophages that partly appeared as foam cells. Individual grading of these inflammatory lesions resulted in a significantly higher score in the bleomycin-treated $\mathrm{Nrf2}^{+/+}$mice compared to their control littermates (Figure 4.3E). In some areas of inflammation, a minimal increase of collagenous fibers was detected. However, a final assessment of these findings was not possible due to some variation during the pharyngeal bleomycin instillation. Interestingly, there was a tendency of less inflammatory lesions in the $\mathrm{Nrf2}^{+/+}$animals treated with quercetin compared to the control group $(2.8 \pm 0.7$ versus $1.9 \pm 0.8)$ although due to the high variation in individual scoring this difference did not reach statistical significance $(p=0.08)$. 

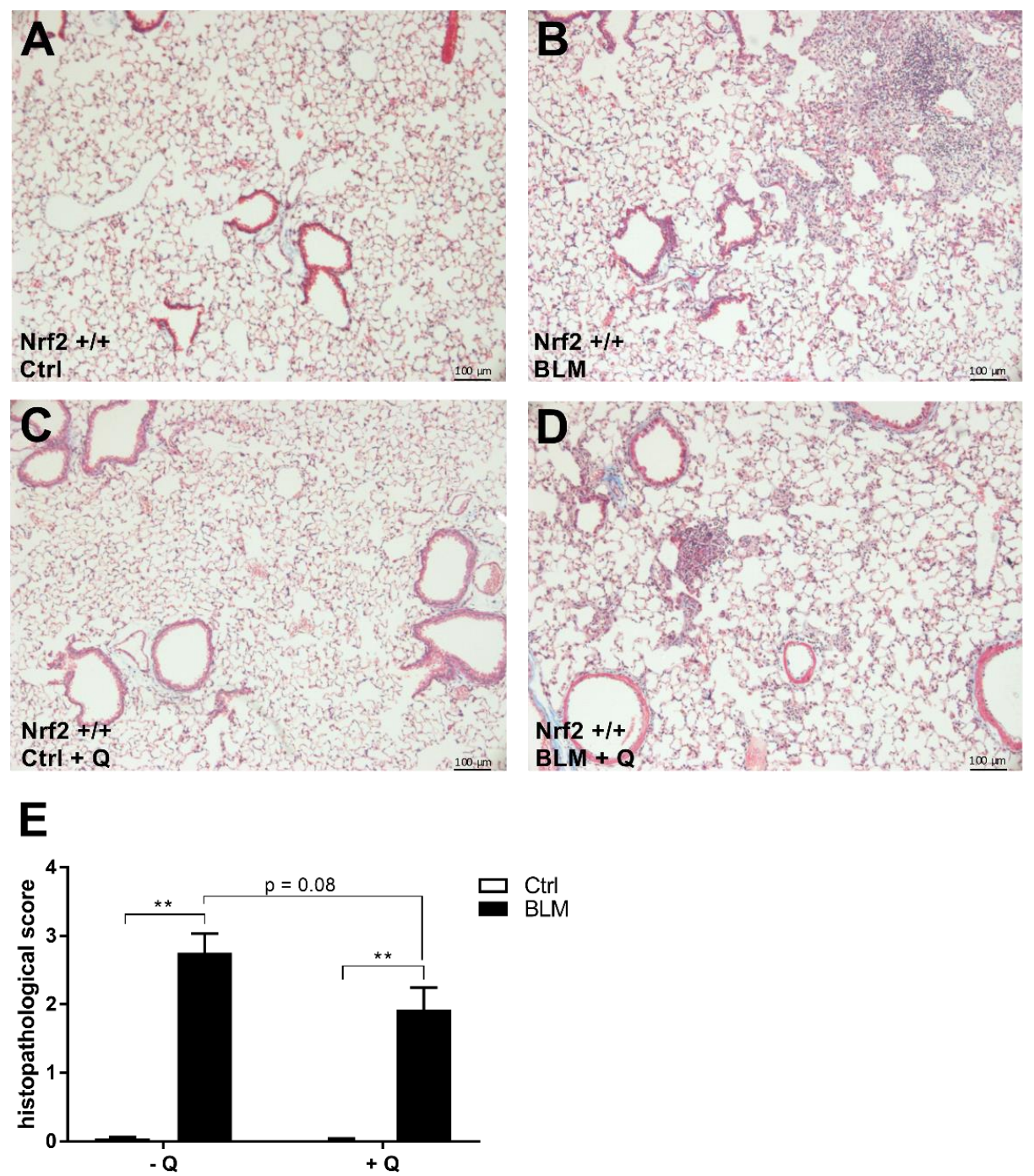

Figure 4.3: Single pharyngeal bleomycin instillation induces inflammatory lesions with some focal increase of collagenous fibers in $\mathrm{Nrf2}^{+/+}$mice. Masson's trichrome stained representative lung sections of control fed (A) $\mathrm{NaCl}(\mathrm{Ctrl})$ and (B) bleomycin (BLM)-instilled $\mathrm{Nrf2}^{+/+}$mice and quercetin-fed (C) Ctrl and (D) BLM-instilled Nrf2 $2^{+/+}$ mice. (E) Histopathological score of the lung tissue ( $n=5-6$ mice/group). Data are expressed as typical example (panel A-D) or mean \pm SEM (panel E); ${ }^{* *} \mathrm{P}<0.01$

In the $\mathrm{Nrf2}^{+/+}$mice, the extracellular matrix (ECM)-related markers collagen (COL1A2) and FN1 supported the histopathology as their gene expression revealed a respectively 3 -fold (figure 4.4A, $\mathrm{P}<0.05$ ) and 10 -fold increase (Figure 4.4B, $\mathrm{P}<0.01$ ) in bleomycin-treated animals compared to the control group. Interestingly, the expression levels of both 
COL1A2 and FN1 were lower in the bleomycin-treated Nrf2+/+ animals fed the quercetin diet compared to those fed the control diet (Figures 4.4A, $\mathrm{P}=0.06$ and Figure $4.4 \mathrm{~B}, \mathrm{P}<0.05$ ). Once the protective effects of quercetin against bleomcyin-induced inflammatory lesions and fibrogenic hallmarks were established, their link with the anti-inflammatory and antioxidative capacities of this dietary antioxidant were evaluated.
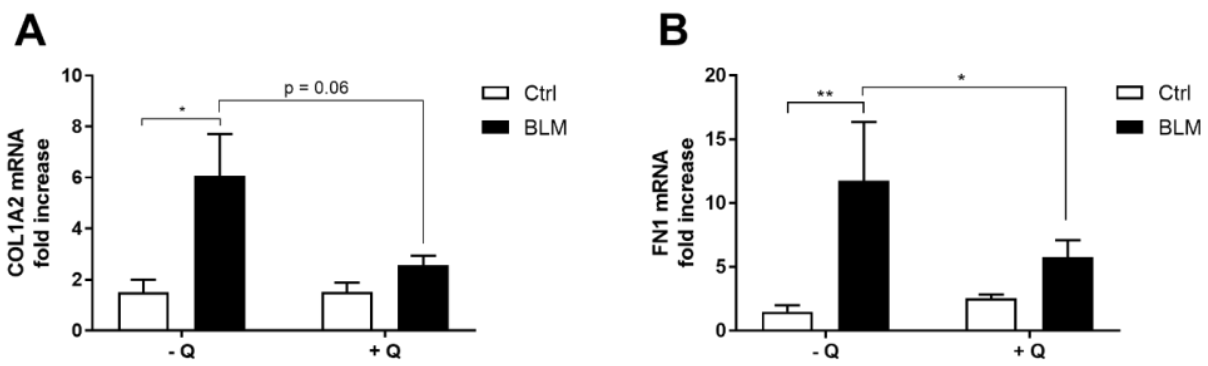

Figure 4.4: Quercetin-enriched diet reduces fibrogenic markers in Nrf2+/+ mice instilled with bleomycin. Effects of bleomycin treatment in the absence or presence of dietary quercetin supplementation are shown for pulmonary expression of (A) COL1A2 and (B) FN1. Data are expressed as mean \pm SEM; $P<0.05 ; * * P<0.01$

\subsection{Quercetin reduces pulmonary yet not systemic BLM-induced inflammation}

As depicted in Figure 4.5, the pulmonary expression of the inflammatory genes KC (panel A) and TNFa (panel C) was two times higher upon bleomycin treatment $(P<0.05$ for KC; n.s. for TNF $\alpha$ ) and restored to baseline levels in the quercetin-fed group ( $<<0.05$ for KC; n.s. for TNF- $\alpha$ ). Systemic plasma levels of these cytokines revealed that KC levels significantly increased from $10.81 \pm 3.58 \mathrm{pg} / \mathrm{ml}$ to $22.88 \pm 4.50 \mathrm{pg} / \mathrm{ml}$ upon bleomycin treatment (Figure 4.5B) whereas TNF $\alpha$ levels remained unaffected by this pro-fibrotic drug (Figure 4.5D). Surprisingly, quercetin supplementation did not exert any systemic antiinflammatory effects as both the plasma KC (Figure 4.4B) and TNF $\alpha$ (Figure 4.5D) levels were unaltered by the antioxidant without and with bleomycin challenge. The other cytokines that were measured in the plasma, were under the detection limit. 
A

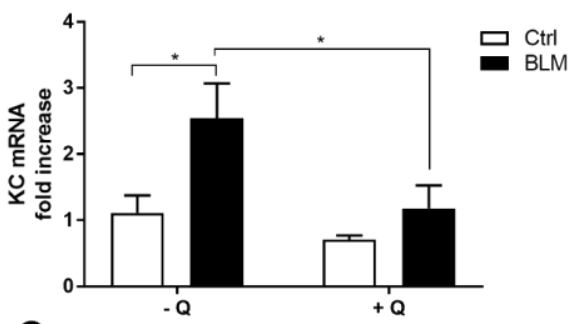

C

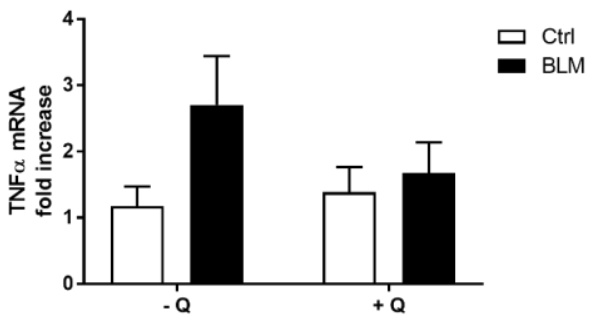

B

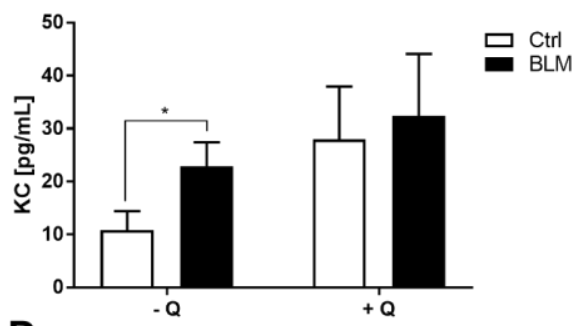

D

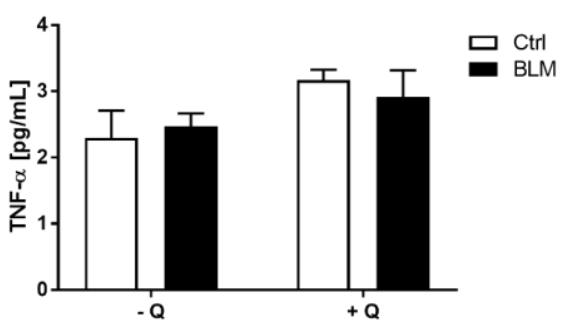

Figure 4.5: Anti-inflammatory effects of quercetin are associated with pulmonary but not systemic antiinflammatory capacities. Pulmonary gene expression and systemic plasma levels of KC (A and C) and TNF- $\alpha$ (B and $\mathrm{D})$ upon bleomycin challenge in the absence or presence of dietary quercetin supplementation. Data are expressed as mean \pm SEM, $*=\mathrm{P}<0.05$

\subsection{Quercetin decreases levels of BLM-induced oxidative parameters}

Since quercetin is a pronounced dietary antioxidant, its influence on both the enzymatic and non-enzymatic antioxidants was evaluated as well as the possible protection it could offer against oxidative DNA damage. Pulmonary expression of the Nrf2-responsive gene yGCS was not affected by either the pro-fibrotic drug or the dietary antioxidant whereas quercetin supplementation did significantly increase the pulmonary expression of Nrf2 $(1.80 \pm 0.43)$ as well as the Nrf2-responsive genes HO-1 (3.82 \pm 1.46$)$, catalase $(5.73 \pm$ $2.60)$ and SOD2 (4.52 \pm 1.35$)$ (Figure 4.6A-E; $\mathrm{P}<0.05)$. Interestingly, bleomycin did not affect the pulmonary expression levels of Nrf2, y-GCS, catalase or SOD2 as reflected in baseline levels in the lungs of animals fed the control diet and enhanced levels again in the animals receiving the quercetin-supplemented diet (Figure 4.6A-D, $P<0.05$ for all genes except SOD2).

The systemic plasma antioxidant defences were evaluated by means of the TEAC assay that analyses the total antioxidant status. Neither quercetin alone nor bleomycin had an effect on the systemic antioxidant status, but once combined they tended to enhance the total plasma antioxidant capacity $(28.07 \pm 5.84 \mathrm{vs} 69.78 \pm 33.66 \mu \mathrm{M})$ but this effect was very variable between individual mice (Figure $4.6 \mathrm{~F}$ ). To assess whether quercetin cannot only boost the antioxidant defences but also offer protection against oxidative damage, DNA-MDA adduct levels were analysed. As anticipated, the pro-fibrotic trigger bleomcyin 
significantly increased DNA-MDA adduct levels ( $83.8 \pm 18.26$ versus $190.2 \pm 20.65$ RAL per $10^{8}$ ) (Figure 4.6G). Dietary supplementation of the antioxidant quercetin restored these elevated DNA-MDA levels back to baseline levels $\left(89.34 \pm 30.78\right.$ RAL per $\left.10^{8}\right)$ although this effect did not reach statistical significance (Figure 4.6G, $P=0.09$ ).

A

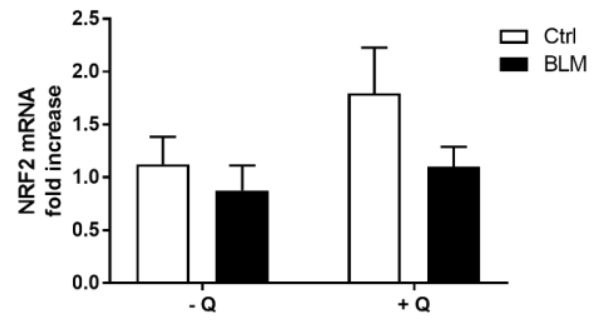

C

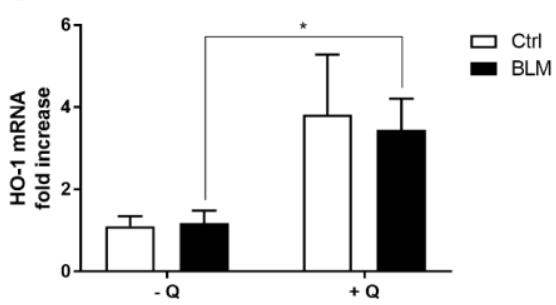

$\mathbf{E}$

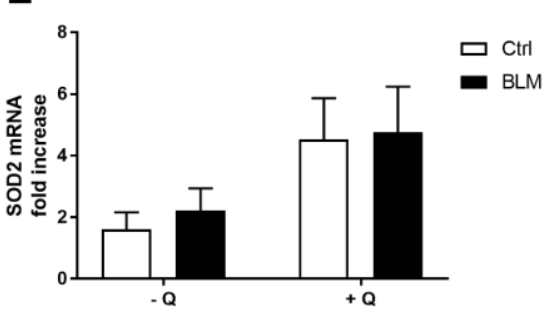

G

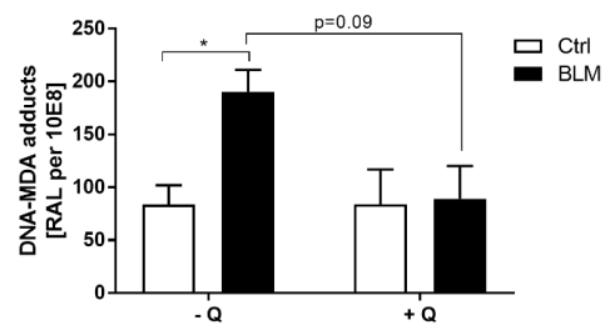

B

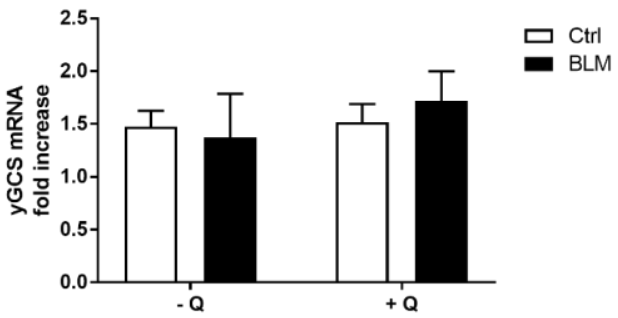

D

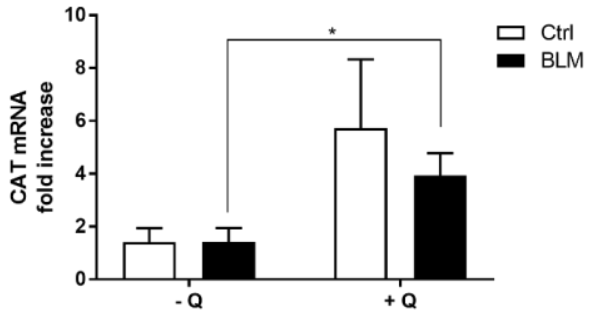

$\mathbf{F}$

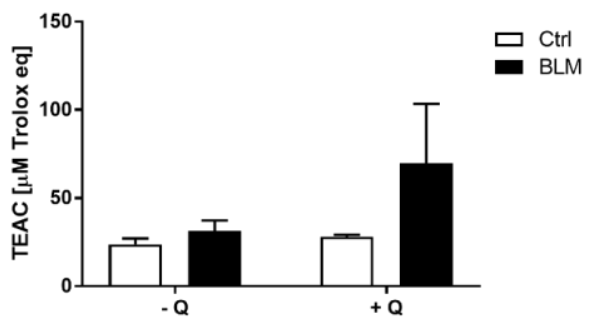

Figure 4.6: Anti-fibrotic effects of quercetin are associated with its anti-oxidative capacities. Pulmonary gene expression levels of (A) Nrf2, (B) YGCS, (C) HO-1, (D) CAT, (E) SOD2, (F) plasma total antioxidant status and (G) DNA-MDA adducts upon bleomycin challenge in the absence or presence of dietary quercetin supplementation. Data are expresses as mean $\pm \mathrm{SEM} ;{ }^{*}=\mathrm{P}<0.05$ 
To investigate, whether the effects of quercetin are dependent on Nrf2, Nrf2 knockout mice $\left(\mathrm{Nrf}^{--}\right)$) were challenged with bleomycin. Histological examination of $\mathrm{H} \& \mathrm{E}$ as well as Masson's Trichrome-stained sections of the lungs revealed a thickening of the airway epithelium in bleomycin-treated $\mathrm{Nrf2}^{-/}$mice (Figure 4.7B) compared to their control littermates (Figure 4.7A). However, no effect of quercetin on either histology or inflammatory lesions (Figure 4.7E) was observed suggesting that quercetin protects against bleomycin-induced pulmonary damage via the induction of Nrf2.
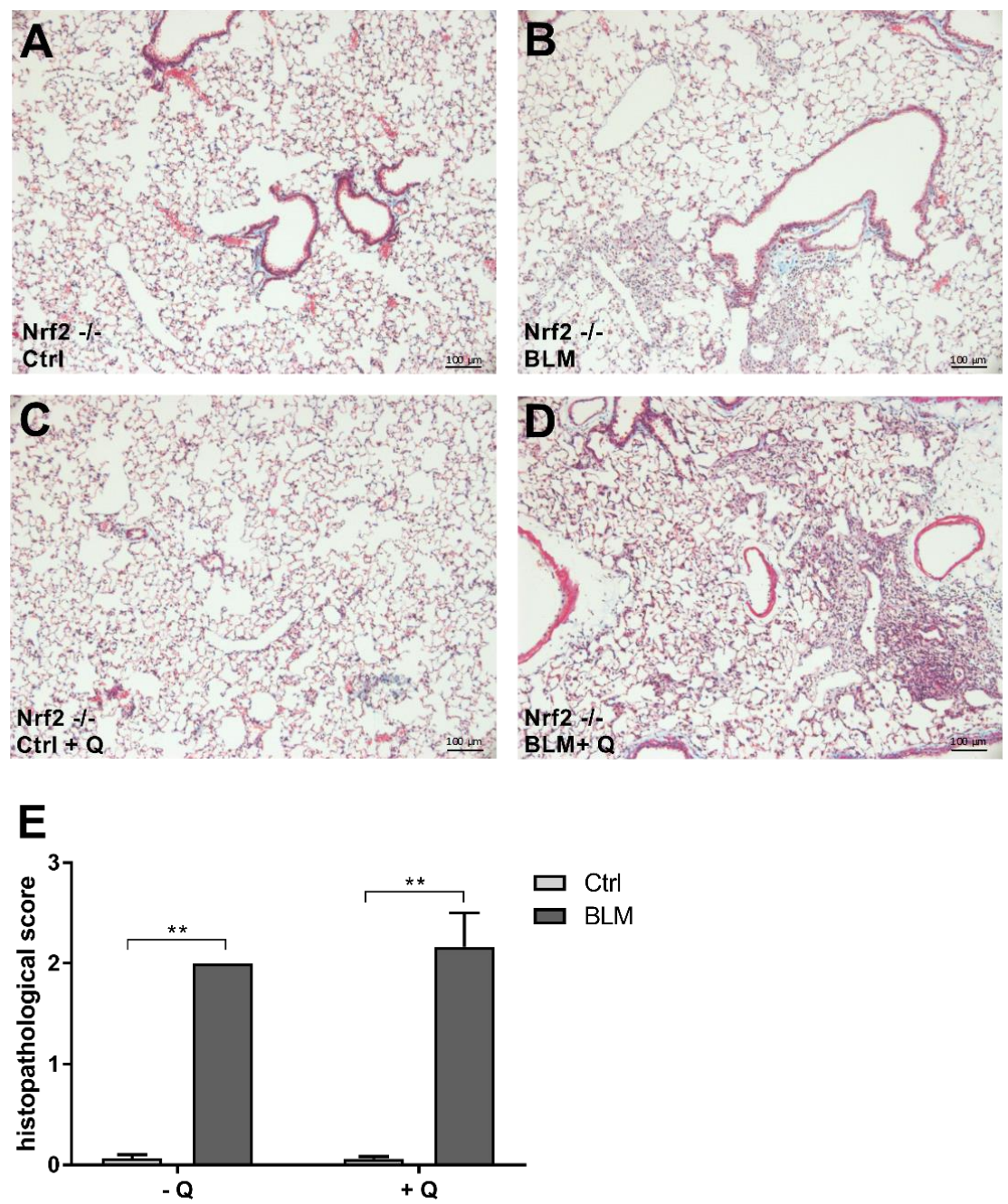

Figure 4.7: Bleomycin-induced inflammatory lesions with some focal increase of collagenous fibers in Nrf2-/mice. Masson's trichrome stained representative lung sections of control-fed (A) $\mathrm{NaCl}(\mathrm{Ctrl})$ and (B) bleomycin (BLM)-instilled Nrf2 $\%$ mice and quercetin-fed (C) Ctrl and (D) BLM-instilled Nrf2 $\%$ mice. (E) Histopathological score of the lung tissue ( $n=5-6$ mice/group). Data are expressed as typical example (panel A-D) or mean \pm SEM (panel $\mathrm{E}) ; * * \mathrm{P}<0.01$ 


\section{Discussion}

IPF is associated with the presence of oxidative stress in the lungs and IPF patients display various markers of oxidative damage $(37,38)$ and a downregulation of several antioxidants (39-41). Restoration of this imbalance between oxidants and antioxidants has been suggested as potential treatment strategy for IPF (42).

In the present study, we provide evidence that dietary quercetin supplementation protects against bleomycin-induced fibrogenesis in mice. Dietary supplementation of quercetin significantly increased the plasma quercetin concentration and enhanced pulmonary antioxidant gene expression without affecting the weight and survival of the mice. Bleomycin instillation induced changes in the lung structure, associated with multifocal inflammatory lesions as well as profibrotic gene expression. This bleomycin-induced pulmonary damage could partly be rescued by dietary quercetin supplementation. Furthermore, quercetin supplementation induced upregulation of Nrf2 and Nrf2-regulated genes and slightly reduced oxidative DNA damage in the bleomycin-challenged lungs. However, histopathologically no clear treatment associated differences could be detected. Antioxidant food supplements such as quercetin have been shown to reduce markers of oxidative stress and fibrotic gene expression in vitro $(28,43)$. At present, this is the first study that showed reduction of bleomycin-induced fibrotic outcomes after ad libitum dietary supplementation of quercetin. Until now, there have only been two studies investigating the effects of solely quercetin supplementation in the absence of any regular drugs or other supplements. However, these studies differed from our study with respect to the animal model applied and the administration mode of quercetin. In agreement with our results, quercetin demonstrated positive effects in two other animal models of pulmonary fibrosis $(44,45)$. Oral quercetin supplementation $(100 \mathrm{mg} / \mathrm{kg}$ bodyweight per day) significantly reduced the hydroxyproline content in the lungs of young rats (10-12 weeks) instilled with bleomycin. This antifibrotic effect was accompanied by decreased levels of MDA, a marker for lipid peroxidation, and enhanced catalase and superoxide dismutase activity (44). In aged mice (>12 months), intraperitoneally quercetin treatment ( $30 \mathrm{mg} / \mathrm{kg}$ every other day) seven days after bleomycin instillation reduced hydroxyproline content in the lungs as well. In this model, the antifibrotic effect of quercetin was combined with decreased pulmonary markers of senescence which in turn reduced the apoptosis resistance of pulmonary fibroblasts (45), indicating that quercetin can also reverse the apoptosis-resistant phenotype of fibroblasts in IPF.

An important hallmark of fibrosis is inflammation and it has been shown before that quercetin has anti-inflammatory capacities and for instance is able to decrease inflammatory cell infiltration such as macrophages, neutrophils and eosinophils (44). Our findings suggest that quercetin exerts an anti-inflammatory effect partly via reducing the pulmonary expression of the inflammatory cytokine KC of which the human functional 
homologue CXCL1 is known to be upregulated in BALF from IPF patients during acute exacerbations (46). Other studies have suggested that bleomycin also increases TNF $\alpha$ (44, 47), which was not observed in our study.

Alleged mode of action underlying this observed defence against bleomcyin-induced pulmonary damage is the antioxidative capacity of quercetin as it is known that bleomycin evokes excessive ROS formation $(36,48)$ and that the lungs are especially vulnerable for alterations in the redox balance $(13,36)$. Indeed, already in the absence of bleomycin, dietary quercetin supplementation led to enhanced pulmonary expression of the redoxsensitive transcription factor Nrf2, as well as Nrf2-responsive antioxidant genes including catalase, HO-1 and y-GCS, previously shown to protect against bleomycin-induced pulmonary fibrosis $(18,22,49)$. In the presence of bleomycin, quercetin supplementation increased the gene expression of most Nrf2-responsive endogenous antioxidants but due to high variation this effect was only statistically significant for catalase and HO-1. This increased antioxidant gene expression was associated with a slightly enhanced total plasma antioxidant capacity in bleomycin-exposed animals fed the quercetin diet compared to animals triggered with only bleomycin, although statistical significance was again not reached due to rather high variation. In line with these increased antioxidative defences due to dietary quercetin supplementation, the DNA-MDA adduct levels were restored to baseline levels in the bleomycin-treated animals fed the quercetin diet compared to their exposed littermates receiving the control diet. Previous studies have revealed that these adduct levels are an important biomarker of oxidative damage with premutagenic activity $(50,51)$, suggesting that lowering these levels might be associated with beneficial health effects.

Our findings indicate that quercetin is well tolerated and taken up but only minimally reduced fibrotic outcomes. Possible explanations for the observed effects of the dietary supplementation being less pronounced than anticipated include i) the dose and mode of quercetin administration, or ii) the way of modulating the redox balance.

It could be possible that the concentration in the lungs was not high enough due to the fact that quercetin is metabolized in the liver upon absorption by the small intestine. The average intake of quercetin in our study was approximately $200 \mathrm{mg}$ quercetin/kilogram BW and resulted in a quercetin concentration of $11.08 \pm 0.73 \mu \mathrm{M}$ in the plasma, $2.97 \pm$ $0.21 \mathrm{nmol} / \mathrm{g}$ in the lungs and $5.05 \pm 1.9 \mathrm{nmol} / \mathrm{g}$ in the $\mathrm{Gl}$ tract which was comparable to a different study performed in rats (33). In this study, the diet was supplemented with $1 \%$ quercetin for 11 weeks, which equals an approximate intake of $500-800 \mathrm{mg} / \mathrm{kg}$ bodyweight (33). The highest concentration of quercetin was found in the plasma and lungs in $(40.5 \mu \mathrm{mol} / \mathrm{L}$ and $5.02 \mathrm{nmol} / \mathrm{g}$ tissue respectively) which is comparable to the findings in our study taking the differences in administered quercetin concentrations into account. However, it is important to note that in both studies the quercetin levels were measured after ex vivo enzymatic hydrolysis whereas the antioxidant is primarily present 
conjugated to methoxy, sulfate and glucoronic acid in tissues. It can yet not be excluded that quercetin is also present in its unconjugated form as especially in the lung relatively high levels of the aglycone were measured, presumably due to high pulmonary deconjugation activity (33). Although most quercetin metabolites possess some antioxidative capacities themselves $(17,52)$, the aglycone is the strongest antioxidative and thus most desirable form of quercetin to expect health beneficial effects from. Consequently, it would be of interest to investigate alternative ways of quercetin administration to prevent the uptake in the GI tract and improve efficiency. Therefore, direct pulmonary administration of quercetin to increase its uptake in the target organ without being metabolized may be more beneficial compared to orally delivered quercetin to exert stronger beneficial effects than currently observed. Another explanation why the beneficial effects of quercetin were only moderate is that the quercetin intake could have varied per individual mouse and per day which could explain the variances within the quercetin group.

Second possible explanation for the moderate effects of the dietary modulation observed in the present study is that the quercetin intervention was too subtle and specific to exert pronounced effects on the pro-fibrotic and especially pro-inflammatory outcomes of the bleomycin challenge. Quercetin is a potent scavenger of hydroxyl radicals and superoxide $(53,54)$ and to a lesser extent of $\mathrm{H}_{2} \mathrm{O}_{2}(17)$. Since NADPH oxidase (NOX) 4 , one of the important pulmonary ROS sources that is upregulated in IPF and promotes alveolar epithelial cell death $(55)$ and fibroblast activation $(56,57)$, mainly produces $\mathrm{H}_{2} \mathrm{O}_{2}$ it can be suggested that quercetin cannot counteract NOX4-induced fibrotic outcomes.

Instead of scavenging excessive production of specific ROS by adding one particular antioxidant to the diet, modulating the redox balance in a more delicate way including a combination of components or a variety of redox-sensitive targets might be more effective to exert beneficial effects in a disease associated with oxidative stress. Such a possible redox-sensitive target is $\mathrm{Nrf2}$, already proven to protect against bleomycininduced pulmonary fibrosis (58) and it has recently been shown that this transcription factor reduces epithelial mesenchymal transition which is a key progression that promotes pulmonary fibrosis (49). The current observation that the histopathology of bleomycinchallenged lungs of $\mathrm{Nrf2}^{-}$mice with and without quercetin diet did not differ, supports the concept that quercetin inhibits fibrogenesis at least partly by boosting the Nrf2 antioxidant pathway (28). In contrast to our findings, a recent study demonstrated no upregulation of Nrf2 genes after quercetin supplementation (45). However, this study has been performed in aged mice indicating that quercetin alone cannot upregulate Nrf2 in this model. It has been indicated before that bronchial epithelial cells from elderly individuals express increased levels of negative Nrf2 regulators (59) suggesting that only inducing Nrf2 is not enough to counteract the development of fibrosis in IPF patients. 
Therefore, the possible protection offered by quercetin has to be elucidated in a different animal model of pulmonary fibrosis.

Interestingly, the currently for IPF therapy approved drug pirfenidone that affects intracellular antioxidants, inflammatory cytokines secretion and collagen synthesis exerts its antioxidative capacities via Nrf2 activation (48). As quercetin is both a ROS scavenger and a potential activator of $(60,61)$, combining regular pirfenidone or nintendanib treatment with this dietary compound may effectively modulate redox balance in IPF and optimize treatment outcome in these patients. Of additional interest to the effectiveness of this combination would be the fact that quercetin and its metabolites exert their effects also via modulating the activity of protein kinases including phosphoinositide 3-kinase (PI3-kinase) (62), Akt/protein kinase B (Akt/PKB) (63) as well as SRC family kinase members SRC, LYN and FYN (64). Intriguingly, a recent study described that quercetin in combination with the $\mathrm{SRC} / \mathrm{ABL}$ protein kinase inhibitor dasatinib reverses bleomycininduced pulmonary fibrosis in aged mice through the reduction of various senescence markers, thereby improving lung function (65). These results further underline that quercetin might exert its maximal health beneficial effects in combination with other antifibrotic drugs, indicating that combination therapy might be more beneficial to counteract ROS-induced damage and the underlying inflammation.

Among the limitations of the present study are the relatively low number of animals per group, the variable pharyngeal bleomycin instillation leading to a rather large spread in the data and the animal model applied. Indeed, the possible protection offered by quercetin has to be elucidated in a different animal model of pulmonary fibrosis applying larger groups as well as a different trigger. Although none of the existing murine models of pulmonary fibrosis really reflects the complex pathogenicity of this disease, specific criticism on the bleomycin model includes the pronounced and rather prolonged inflammatory response whereas this process is thought to only play a minor role in IPF development $(36,66)$. However, it is thought that inflammation may play a role, though potentially to a different extent, in both murine and human pulmonary fibrosis, indicating that more research is needed to improve understanding the involvement of inflammation in IPF (66). Additionally, different time points and doses for bleomycin administration have been reported in literature, resulting in different degrees of fibrotic damage and inflammation and hampering the straight-forward comparison between various studies (36).

In conclusion, we demonstrated that dietary quercetin supplementation reduces profibrotic gene expression after bleomycin instillation in lungs of mice. Additionally, a tendency of decreased inflammatory lesions was seen. However, the observed protective effects were only moderate indicating that scavenging ROS is not enough to counteract the development of pulmonary fibrosis. 


\section{Acknowledgements}

The authors wish to thank Maja Hullman, Julia Kolling, Christel Weishaupt and Damien van Berlo for their technical support. The work presented in this article was financially supported by an ERS/Marie Curie Fellowship granted to Agnes Boots. 


\section{References}

1. Nalysnyk L, Cid-Ruzafa J, Rotella P, Esser D. Incidence and prevalence of idiopathic pulmonary fibrosis: review of the literature. Eur Respir Rev. 2012;21(126):355-61.

2. Kaur A, Mathai SK, Schwartz DA. Genetics in Idiopathic Pulmonary Fibrosis Pathogenesis, Prognosis, and Treatment. Front Med (Lausanne). 2017;4:154.

3. Meiners S, Eickelberg O, Konigshoff M. Hallmarks of the ageing lung. Eur Respir J. 2015;45(3):807-27.

4. Raghu G, Collard HR, Egan JJ, Martinez FJ, Behr J, Brown KK, et al. An Official ATS/ERSARS/ALAT Statement: Idiopathic Pulmonary Fibrosis: Evidence-based Guidelines for Diagnosis and Management. Am J Resp Crit Care. 2011;183(6):788-824.

5. King TE, Jr., Bradford WZ, Castro-Bernardini S, Fagan EA, Glaspole I, Glassberg MK, et al. A phase 3 trial of pirfenidone in patients with idiopathic pulmonary fibrosis. $\mathrm{N}$ Engl J Med. 2014;370(22):2083-92.

6. Macagno F, Varone F, Leone PM, Mari PV, Panico L, Berardini L, et al. New treatment directions for IPF: current status of ongoing and upcoming clinical trials. Expert Rev Respir Med. 2017;11(7):533-48.

7. Richeldi L, du Bois RM, Raghu G, Azuma A, Brown KK, Costabel U, et al. Efficacy and safety of nintedanib in idiopathic pulmonary fibrosis. N Engl J Med. 2014;370(22):2071-82.

8. Canestaro WJ, Forrester SH, Raghu G, Ho L, Devine BE. Drug Treatment of Idiopathic Pulmonary Fibrosis: Systematic Review and Network Meta-Analysis. Chest. 2016;149(3):756-66.

9. Wolters PJ, Collard HR, Jones KD. Pathogenesis of idiopathic pulmonary fibrosis. Annu Rev Pathol. 2014;9:157-79.

10. Fois AG, Paliogiannis $P$, Sotgia $S$, Mangoni AA, Zinellu E, Pirina $P$, et al. Evaluation of oxidative stress biomarkers in idiopathic pulmonary fibrosis and therapeutic applications: a systematic review. Respir Res. 2018;19(1):51.

11. Hecker L. Mechanisms and consequences of oxidative stress in lung disease: therapeutic implications for an aging populace. Am J Physiol Lung Cell Mol Physiol. 2018;314(4):L642-L53.

12. Bartoli ML, Novelli F, Costa F, Malagrino L, Melosini L, Bacci E, et al. Malondialdehyde in exhaled breath condensate as a marker of oxidative stress in different pulmonary diseases.

Mediators Inflamm. 2011;2011:891752.

13. Bast A, Weseler AR, Haenen GR, den Hartog GJ. Oxidative stress and antioxidants in interstitial lung disease. Curr Opin Pulm Med. 2010;16(5):516-20.

14. Kanoh S, Kobayashi H, Motoyoshi K. Exhaled ethane: an in vivo biomarker of lipid peroxidation in interstitial lung diseases. Chest. 2005;128(4):2387-92.

15. Malerba M, Radaeli A, Ragnoli B, Airo P, Corradi M, Ponticiello A, et al. Exhaled nitric oxide levels in systemic sclerosis with and without pulmonary involvement. Chest. 2007;132(2):575-80.

16. Ding $Q$, Luckhardt T, Hecker L, Zhou Y, Liu G, Antony VB, et al. New insights into the pathogenesis and treatment of idiopathic pulmonary fibrosis. Drugs. 2011;71(8):981-1001.

17. Boots AW, Haenen GR, Bast A. Health effects of quercetin: from antioxidant to nutraceutical. Eur J Pharmacol. 2008;585(2-3):325-37.

18. Cho HY, Reddy SP, Kleeberger SR. Nrf2 defends the lung from oxidative stress. Antioxidants \& redox signaling. 2006;8(1-2):76-87.

19. Walters DM, Cho HY, Kleeberger SR. Oxidative stress and antioxidants in the pathogenesis of pulmonary fibrosis: a potential role for Nrf2. Antioxid Redox Signal. 2008;10(2):321-32.

20. Rubio V, Valverde M, Rojas E. Effects of atmospheric pollutants on the Nrf2 survival pathway. Environ Sci Pollut Res Int. 2010;17(2):369-82. 
21. Blake DJ, Singh A, Kombairaju P, Malhotra D, Mariani TJ, Tuder RM, et al. Deletion of Keap1 in the lung attenuates acute cigarette smoke-induced oxidative stress and inflammation. Am J Respir Cell Mol Biol. 2010;42(5):524-36.

22. Cho HY, Reddy SP, Yamamoto M, Kleeberger SR. The transcription factor NRF2 protects against pulmonary fibrosis. FASEB J. 2004;18(11):1258-60.

23. Rangasamy T, Guo J, Mitzner WA, Roman J, Singh A, Fryer AD, et al. Disruption of Nrf2 enhances susceptibility to severe airway inflammation and asthma in mice. J Exp Med. 2005;202(1):47-59.

24. Markart $\mathrm{P}$, Luboeinski T, Korfei M, Schmidt R, Wygrecka M, Mahavadi $\mathrm{P}$, et al. Alveolar oxidative stress is associated with elevated levels of nonenzymatic low-molecular-weight antioxidants in patients with different forms of chronic fibrosing interstitial lung diseases. Antioxid Redox Signal. 2009;11(2):227-40.

25. Demedts M, Behr J, Buhl R, Costabel U, Dekhuijzen R, Jansen HM, et al. High-dose acetylcysteine in idiopathic pulmonary fibrosis. N Engl J Med. 2005;353(21):2229-42.

26. Cortijo J, Cerda-Nicolas M, Serrano A, Bioque G, Estrela JM, Santangelo F, et al. Attenuation by oral $\mathrm{N}$-acetylcysteine of bleomycin-induced lung injury in rats. Eur Respir J. 2001;17(6):1228-35.

27. Hertog MG, Hollman PC, Katan MB, Kromhout D. Intake of potentially anticarcinogenic flavonoids and their determinants in adults in The Netherlands. Nutr Cancer. 1993;20(1):21-9.

28. Veith C, Drent M, Bast A, van Schooten FJ, Boots AW. The disturbed redox-balance in pulmonary fibrosis is modulated by the plant flavonoid quercetin. Toxicol Appl Pharmacol. 2017;336:40-8.

29. Boots AW, Drent M, de Boer VC, Bast A, Haenen GR. Quercetin reduces markers of oxidative stress and inflammation in sarcoidosis. Clin Nutr. 2011;30(4):506-12.

30. Numazawa S, Yoshida T. Nrf2-dependent gene expressions: a molecular toxicological aspect. J Toxicol Sci. 2004;29(2):81-9.

31. Tamasi V, Jeffries JM, Arteel GE, Falkner KC. Ebselen augments its peroxidase activity by inducing nrf-2-dependent transcription. Arch Biochem Biophys. 2004;431(2):161-8.

32. Itoh K, Chiba T, Takahashi S, Ishii T, Igarashi K, Katoh Y, et al. An Nrf2/small Maf heterodimer mediates the induction of phase II detoxifying enzyme genes through antioxidant response elements. Biochem Biophys Res Commun. 1997;236(2):313-22.

33. de Boer VC, Dihal AA, van der Woude H, Arts IC, Wolffram S, Alink GM, et al. Tissue distribution of quercetin in rats and pigs. J Nutr. 2005;135(7):1718-25.

34. Fischer MA, Gransier TJ, Beckers LM, Bekers O, Bast A, Haenen GR. Determination of the antioxidant capacity in blood. Clin Chem Lab Med. 2005;43(7):735-40.

35. Peluso M, Munnia A, Ceppi M, Giese RW, Catelan D, Rusconi F, et al. Malondialdehydedeoxyguanosine and bulky DNA adducts in schoolchildren resident in the proximity of the Sarroch industrial estate on Sardinia Island, Italy. Mutagenesis. 2013;28(3):315-21.

36. Moore BB, Hogaboam CM. Murine models of pulmonary fibrosis. Am J Physiol Lung Cell Mol Physiol. 2008;294(2):L152-60.

37. Psathakis K, Mermigkis D, Papatheodorou G, Loukides S, Panagou P, Polychronopoulos V, et al. Exhaled markers of oxidative stress in idiopathic pulmonary fibrosis. Eur J Clin Invest. 2006;36(5):362-7.

38. Lenz AG, Costabel U, Maier KL. Oxidized BAL fluid proteins in patients with interstitial lung diseases. The European respiratory journal. 1996;9(2):307-12.

39. Cantin AM, Hubbard RC, Crystal RG. Glutathione deficiency in the epithelial lining fluid of the lower respiratory tract in idiopathic pulmonary fibrosis. Am Rev Respir Dis. 1989;139(2):370-2.

40. Kinnula VL, Hodgson UA, Lakari EK, Tan RJ, Sormunen RT, Soini YM, et al. Extracellular superoxide dismutase has a highly specific localization in idiopathic pulmonary fibrosis/usual interstitial pneumonia. Histopathology. 2006;49(1):66-74. 
41. Odajima N, Betsuyaku T, Nagai K, Moriyama C, Wang DH, Takigawa T, et al. The role of catalase in pulmonary fibrosis. Respir Res. 2010;11:183.

42. Kurundkar A, Thannickal VJ. Redox mechanisms in age-related lung fibrosis. Redox Biol. 2016;9:67-76.

43. Nakamura T, Matsushima M, Hayashi Y, Shibasaki M, Imaizumi K, Hashimoto N, et al. Attenuation of transforming growth factor-beta-stimulated collagen production in fibroblasts by quercetin-induced heme oxygenase-1. Am J Respir Cell Mol Biol. 2011;44(5):614-20.

44. Verma R, Kushwah L, Gohel D, Patel M, Marvania T, Balakrishnan S. Evaluating the Ameliorative Potential of Quercetin against the Bleomycin-Induced Pulmonary Fibrosis in Wistar Rats. Pulm Med. 2013;2013:921724.

45. Hohmann MS, Habiel DM, Coelho AL, Verri WA, Jr., Hogaboam CM. Quercetin Enhances Ligand-induced Apoptosis in Senescent Idiopathic Pulmonary Fibrosis Fibroblasts and Reduces Lung Fibrosis In Vivo. Am J Respir Cell Mol Biol. 2019;60(1):28-40.

46. Schupp JC, Binder H, Jager B, Cillis G, Zissel G, Muller-Quernheim J, et al. Macrophage activation in acute exacerbation of idiopathic pulmonary fibrosis. PLoS One. 2015;10(1):e0116775.

47. Nair MP, Mahajan S, Reynolds JL, Aalinkeel R, Nair H, Schwartz SA, et al. The flavonoid quercetin inhibits proinflammatory cytokine (tumor necrosis factor alpha) gene expression in normal peripheral blood mononuclear cells via modulation of the NF-kappa beta system. Clin Vaccine Immunol. 2006;13(3):319-28.

48. Liu Y, Lu F, Kang L, Wang Z, Wang Y. Pirfenidone attenuates bleomycin-induced pulmonary fibrosis in mice by regulating Nrf2/Bach1 equilibrium. BMC Pulm Med. 2017;17(1):63.

49. Zhang Z, Qu J, Zheng C, Zhang P, Zhou W, Cui W, et al. Nrf2 antioxidant pathway suppresses Numb-mediated epithelial-mesenchymal transition during pulmonary fibrosis. Cell Death Dis. 2018;9(2):83.

50. Munnia A, Bonassi S, Verna A, Quaglia R, Pelucco D, Ceppi M, et al. Bronchial malondialdehyde DNA adducts, tobacco smoking, and lung cancer. Free Radic Biol Med. 2006;41(9):1499-505.

51. Godschalk R, Nair J, van Schooten FJ, Risch A, Drings P, Kayser K, et al. Comparison of multiple DNA adduct types in tumor adjacent human lung tissue: effect of cigarette smoking. Carcinogenesis. 2002;23(12):2081-6.

52. Lemanska K, van der Woude H, Szymusiak H, Boersma MG, Gliszczynska-Swiglo A, Rietjens $\mathrm{IM}$, et al. The effect of catechol O-methylation on radical scavenging characteristics of quercetin and luteolin--a mechanistic insight. Free Radic Res. 2004;38(6):639-47.

53. Chen YT, Zheng RL, Jia ZJ, Ju Y. Flavonoids as superoxide scavengers and antioxidants. Free Radic Biol Med. 1990;9(1):19-21.

54. Wilms LC, Kleinjans JC, Moonen EJ, Briede JJ. Discriminative protection against hydroxyl and superoxide anion radicals by quercetin in human leucocytes in vitro. Toxicol In Vitro. 2008;22(2):301-7.

55. Carnesecchi S, Deffert C, Donati Y, Basset O, Hinz B, Preynat-Seauve O, et al. A key role for NOX4 in epithelial cell death during development of lung fibrosis. Antioxidants \& redox signaling. 2011;15(3):607-19.

56. Amara N, Goven D, Prost F, Muloway R, Crestani B, Boczkowski J. NOX4/NADPH oxidase expression is increased in pulmonary fibroblasts from patients with idiopathic pulmonary fibrosis and mediates TGFbeta1-induced fibroblast differentiation into myofibroblasts. Thorax. 2010;65(8):733-8.

57. Hecker L, Vittal R, Jones T, Jagirdar R, Luckhardt TR, Horowitz JC, et al. NADPH oxidase-4 mediates myofibroblast activation and fibrogenic responses to lung injury. Nat Med. 2009;15(9):1077-81.

58. Kikuchi N, Ishii Y, Morishima Y, Yageta Y, Haraguchi N, Itoh K, et al. Nrf2 protects against pulmonary fibrosis by regulating the lung oxidant level and Th1/Th2 balance. Respir Res. 2010;11:31. 
59. Zhou L, Zhang H, Davies KJA, Forman HJ. Aging-related decline in the induction of Nrf2regulated antioxidant genes in human bronchial epithelial cells. Redox Biol. 2018;14:35-40.

60. Swamy SM, Rajasekaran NS, Thannickal VJ. Nuclear Factor-Erythroid-2-Related Factor 2 in Aging and Lung Fibrosis. Am J Pathol. 2016;186(7):1712-23.

61. Tanigawa S, Fujii M, Hou DX. Action of Nrf2 and Keap1 in ARE-mediated NQO1 expression by quercetin. Free Radic Biol Med. 2007;42(11):1690-703.

62. Matter WF, Brown RF, Vlahos CJ. The inhibition of phosphatidylinositol 3-kinase by quercetin and analogs. Biochem Biophys Res Commun. 1992;186(2):624-31.

63. Spencer JP, Rice-Evans C, Williams RJ. Modulation of pro-survival Akt/protein kinase B and ERK1/2 signaling cascades by quercetin and its in vivo metabolites underlie their action on neuronal viability. J Biol Chem. 2003;278(37):34783-93.

64. Navarro-Nunez L, Lozano ML, Martinez C, Vicente V, Rivera J. Effect of quercetin on platelet spreading on collagen and fibrinogen and on multiple platelet kinases. Fitoterapia. 2010;81(2):75-80.

65. Schafer MJ, White TA, lijima K, Haak AJ, Ligresti G, Atkinson EJ, et al. Cellular senescence mediates fibrotic pulmonary disease. Nat Commun. 2017;8:14532.

66. Williamson JD, Sadofsky LR, Hart SP. The pathogenesis of bleomycin-induced lung injury in animals and its applicability to human idiopathic pulmonary fibrosis. Exp Lung Res. 2015;41(2):5773. 



\title{
Chapter 5
}

\section{Profibrotic signaling by TGF- $\beta 1$ involves \\ NADPH oxidase 4- dependent activation of the tyrosine kinase FYN}

\begin{abstract}
Carmen Veith, Milena Hristova, Karamatullah Danyal, Aida Habibovic, John E. McDonough, Bart M. Vanaudenaerde, Frederik-Jan van Schooten, Agnes W. Boots and Albert van der Vliet.
\end{abstract}

Submitted 


\section{Abstract}

Idiopathic pulmonary fibrosis (IPF) is characterized by a disturbed redox balance and increased production of reactive oxygen species (ROS), which is believed to contribute to epithelial injury and fibrotic lung scarring. The main pulmonary sources of ROS include mitochondria and NADPH oxidases (NOXs), of which the NOX4 isoform has been implicated in IPF. Non-receptor SRC tyrosine kinases are involved in various signaling pathways important for cellular homeostasis and are often dysregulated in lung diseases. Indeed, SRC family kinases (SFK) are activated by the profibrotic transforming growth factor- $\beta$ (TGF- $\beta$ ) and are thought to contribute to pulmonary fibrosis. However, it is unclear which SFK member mediates epithelial injury and profibrotic responses and how TGF- $\beta$ leads to SFK activation. Here, we demonstrate that TGF- $\beta 1$ induces rapid activation of the SRC kinase FYN in human bronchial epithelial cells, which subsequently induces mitochondrial ROS (mtROS) production, genetic damage shown by the DNA damage marker $\gamma \mathrm{H} 2 \mathrm{AX}$, and increased expression of profibrotic genes. Moreover, TGF- $\beta 1$-induced activation of FYN and mtROS production involve initial activation of NOX4 and direct cysteine oxidation of FYN. NOX4 expression in lung tissues of IPF patients is positively correlated with disease severity, although FYN expression is significantly downregulated in IPF and does not correlate with disease severity. Collectively, our findings highlight a critical role for NOX4 and FYN in TGF- $\beta 1$-induced mtROS production, DNA damage response, and induction of profibrotic genes in bronchial epithelial cells, and suggest that dysregulated expression and activation of NOX4 and FYN may contribute to the pathogenesis of pulmonary fibrosis.

Keywords: Idiopathic pulmonary fibrosis; NOX4; SRC kinases; FYN; redox signaling; mitochondria 


\section{Introduction}

Idiopathic pulmonary fibrosis (IPF) is a chronic, progressive interstitial lung disease with a median survival of 3-5 years (1). Although its exact cause is still unknown, its pathology is believed to be driven by continuous epithelial injury and altered fibroblast biology causing aberrant wound healing and scarring of the lung tissue, eventually leading to death from respiratory failure (2). With the exception of two drug therapies approved by the Food and Drug Administration (FDA), i.e. pirfenidone and nintedanib, there are no effective treatment options for IPF (3). Importantly, these approved drugs primarily slow down the progression of the disease but do not reverse fibrosis nor improve lung function (3). Therefore, there is an urgent need to develop new therapeutic strategies that are more effective in combatting the dreadful consequences of this disease.

A key process in the pathology of IPF is augmented or dysregulated production of reactive oxygen species (ROS) leading to oxidative stress $(4,5)$. Oxidative stress causes lung epithelial cell death and lung fibroblast differentiation, two key processes in the development of IPF (6). Although ROS can serve as signaling molecules that regulate important cell functions, high levels of ROS can also be harmful. Indeed, a chronic overload of ROS, as occurs in the setting of chronic age-related lung diseases such as IPF, causes progressive oxidative damage to DNA, proteins and lipids, and the progression of these chronic lung diseases has been linked to increased markers of DNA damage which may underlay genetic instability $(7,8)$. Although oxidative stress is widely believed to contribute to the development of IPF, therapeutic approaches to mitigate the effects of ROS with antioxidant strategies, such as $\mathrm{N}$-acetylcysteine, have not been successful (9), although alternative redox-based approaches have shown promise (10). Cellular sources of ROS include the mitochondrial electron transport chain (ETC), as well as specific intracellular enzymes including NADPH oxidases (NOXs). With respect to the latter, the isoform NOX4, which generates $\mathrm{H}_{2} \mathrm{O}_{2}$ and is primarily localized in intracellular membranes of mitochondria, endoplasmic reticulum and the nucleus (11), is highly upregulated in the lungs of IPF patients (12-14). Moreover, it has been shown that epithelial injury induces gene expression of NOX4, resulting in the release of profibrotic cytokines including transforming growth factor (TGF)- $\beta$, thereby further promoting fibrosis $(12,15)$. As a result, selective inhibitors of NOX4 are being evaluated as potential therapeutic strategies for IPF (16).

The growth factor TGF- $\beta 1$ is widely recognized as a main driver in the development of IPF (17). Although TGF- $\beta$ is important in wound healing responses, chronic TGF- $\beta$ production due to repetitive injury of the alveolar epithelium contributes to epithelial injury as well as features of epithelial-to-mesenchymal transition (EMT), thereby leading to aberrant wound healing responses. Additionally, TGF- $\beta$ induces proliferation and differentiation of (myo)fibroblasts, eventually leading to excessive collagen deposition and remodeling of 
the lung tissue $(17,18)$. TGF- $\beta$ is capable of inducing NOX4 expression (19) as well as mitochondrial ROS production (20), thereby promoting redox imbalance and profibrotic responses, although questions remain regarding the precise molecular mechanisms involved.

Recent studies have implicated non-receptor SRC family kinases (SFK) in TGF- $\beta$-mediated cell responses, and in the development of lung fibrosis $(21,22)$. SFK's such as SRC, FYN and YES are involved in various signaling pathways important for cellular homeostasis, including cell differentiation and proliferation $(23,24)$ and are often dysregulated in the pathogenesis of IPF (25). SFKs have also been implicated in EMT $(26,27)$, an important feature of aberrant wound healing. Moreover, general pharmacological inhibition of SFKs can inhibit collagen deposition and fibrotic lesions in experimental models of $\operatorname{IPF}(21,28)$. However, the importance of specific SRC kinase isoforms in the epithelial injury and profibrotic responses are largely unknown. In addition, while SFKs are primarily regulated by (de)phosphorylation events $(29,30)$, their activation is also subject to redox-dependent mechanisms, independent of the dephosphorylation of Tyr527 (21, 29, 31, 32). However, the specific oxidative mechanisms and ROS source(s) involved in SFK activation in the context of TGF- $\beta$ signaling are not clear. The present study was conducted to explore the importance of SFKs and redox-dependent mechanisms in the profibrotic actions of TGF- $\beta$ in lung epithelial cells. Our results indicate the importance of NOX4 in TGF- $\beta$-mediated activation of the SFK FYN, which subsequently mediates mitochondrial ROS production, DNA damage responses, and profibrotic gene expression, as critical events in IPF pathology. 


\section{Materials and Methods}

\subsection{Cell culture and treatment}

Immortalized human bronchial epithelial (HBE1) cells were maintained in DMEM/F12 media (Invitrogen, Carlsbad, CA) supplemented with $1 \mathrm{ng} / \mathrm{ml}$ cholera toxin (List Biological Laboratories, Campbell, CA), $10 \mathrm{ng} / \mathrm{mL}$ epidermal growth factor (Calbiochem, San Diego, $\mathrm{CA}$ ), $5 \mu \mathrm{g} / \mathrm{mL}$ insulin (Sigma, St. Louis, MO), $5 \mu \mathrm{g} / \mathrm{mL}$ transferrin (Sigma), $0.1 \mu \mathrm{M}$ dexamethasone (Sigma), $15 \mu \mathrm{g} / \mathrm{mL}$ bovine pituitary extract (Invitrogen), $0.5 \mathrm{mg} / \mathrm{mL}$ bovine serum albumin (Invitrogen) and $50 \mathrm{U} / 50 \mu \mathrm{g} / \mathrm{mL}$ penicillin/streptomycin (Invitrogen), as described previously (33). Cells were seeded in 24-well plates or chamber slides unless otherwise indicated and grown to confluence, and were starved in EGF-free media for 24 hrs prior to experiments. Cells were stimulated with $10 \mathrm{ng} / \mathrm{mL}$ TGF- $\beta 1$ (R\&D Systems, Minneapolis, MN) and harvested at appropriate time points for analysis of SRC activation, ROS production, NADPH oxidase expression, DNA damage response, and pro-fibrotic gene expression. Where indicated, the pharmacological inhibitor PP2 (1 $\mu \mathrm{M}$, Sigma) and the mitochondria-targeted antioxidant MitoQ ( $1 \mu \mathrm{M}$, Sigma) were administered 20 min before cell stimulation. At appropriate times, cells were harvested for RNA extraction for RT-PCR analyses or preparation of cell lysates for Western blot analyses, or for other assays described below.

\subsection{Small interfering RNA silencing}

At $60 \%$ to $70 \%$ confluency, HBE1 cells were transfected with ON-TARGETplus SMARTpool small interfering RNAs (siRNAs) targeted against SRC, FYN, NOX4 or non-targeting siRNA Pool\#1 as a control, using DharmaFECT transfection reagent (Dharmacon, Lafayette, CO) according to the manufacturer's instructions, $72 \mathrm{hrs}$ before experimentation.

\subsection{RNA isolation and semi quantitative reverse transcription (RT)-PCR}

RNA was isolated and purified using the RNeasy mini kit (Qiagen, Valencia, CA) according to the manufacturer's instructions. The RNA concentration was determined using a Nanodrop spectrophotometer (Thermo Scientific, Waltham, MA). cDNA was synthesized from $500 \mathrm{ng}$ isolated RNA using IScript (Biorad, Hercules, CA) according to the manufacturer's instructions. RT-PCR was performed using SYBR Green PCR Supermix (BioRad) with $1 \mu \mathrm{L}$ of CDNA and $0.5 \mu \mathrm{mol} / \mathrm{L}$ predesigned primers and PCR amplifications were carried out for up to 40 cycles of denaturation $\left(95^{\circ} \mathrm{C}, 15 \mathrm{sec}\right)$, annealing $\left(57^{\circ} \mathrm{C}, 15\right.$ sec) and extension $\left(60^{\circ} \mathrm{C}, 45 \mathrm{sec}\right)$ for selected genes (Table 1$)$. The gene expression was normalized to the house keeping gene glyceraldehyde-3-phosphate dehydrogenase (GAPDH) and quantified using the $\Delta \Delta$ cycle threshold $\left(C_{T}\right)$ method. 
Table 5.1: Forward and reverse RT-PCR primer sequences for indicated genes.

\begin{tabular}{|l|l|l|}
\hline Gene of interest & Forward primer sequence & Reverse primer sequence \\
\hline GAPDH & GAAGGCTGGGGCTCATTTG & AGGCTGTTGTCATACTTCTCATGG \\
\hline COL1A1 & GGACACAGAGGTTTCAGTGG & CCAGTAGCACCATCATTTCC \\
\hline VIM & GACAATGCGTCTCTGGCACGTCTT & TCCTCCGCCTCCTGCAGGTTCTT \\
\hline FN1 & AGTGGGAGACCTCGAGAAGA & ACTGTGACAGCAGGAGCATC \\
\hline FYN & AGCAAGCAAATGGCTGATAC & TGAGAAAGTTTAGCGGGTTG \\
\hline YES & AAGGACCCTGATGAAAGACC & TCTGGGATTCCAGTTTACCA \\
\hline SRC & GGG TGA TGTTTGACCTTCAG & TAGGCACTCTTTTCCCTCCT \\
\hline NOX1 & CTTGCCTCCATTCTCTCCAG & CACTCCAGTGAGACCAGCAA \\
\hline NOX2 & AATCATCCATGCCACCAT TT & TCGAAATCTGCTGTCTTCC \\
\hline NOX4 & TGGCAAGAGAACAGACCTGA & TGGGTCCACAACAGAAAACA \\
\hline DUOX1 & TTCACGCAGCTCTGTGTCAA & AGGGACAGATCATATCCTGGCT \\
\hline DUOX2 & ACGCAGCTCTGTGTCAAAGGT & TGATGAACGAGACTCGACAGC \\
\hline
\end{tabular}

\subsection{Analysis of cellular ROS production}

\section{2,7-Dichlorodihydrofluorescein diacetate assay}

For analysis of intracellular ROS production, cells were incubated with $10 \mu \mathrm{M}$ using 2,7dichlorofluorescein diacetate (DCF-DA; Sigma) for $20 \mathrm{~min}$ at $37^{\circ} \mathrm{C}$ and washed with PBS and placed in fresh media for stimulation with $10 \mathrm{ng} / \mathrm{mL}$ TGF- $\beta 1$. DCF fluorescence was measured with excitation at $485 \mathrm{~nm}$ and emission at $525 \mathrm{~nm}$ using a 96 -well plate reader.

\section{Amplex Red assay}

Extracellular $\mathrm{H}_{2} \mathrm{O}_{2}$ production was measured in cell culture supernatants using Amplex ${ }^{\circledR}$ Red (Invitrogen) according to the manufactures instructions. Briefly, Amplex Red reacts with $\mathrm{H}_{2} \mathrm{O}_{2}$ in a 1:1 stoichiometry to produce the red-fluorescent oxidation product, resorufin, which can be detected fluorometrically (530-560 nm excitation; $590 \mathrm{~nm}$ emission).

\section{Mitochondrial ROS production}

To evaluate mitochondrial ROS production, cells were plated on glass bottom dishes (MatTek, Ashland, MA) and preloaded for 20 minutes with the fluorescent probe MitosOX ( $5 \mu \mathrm{M}$, Invitrogen) dissolved in HBSS and ROS production was followed for 20 minutes by live-cell imaging with a 40X objective using a Zeiss LSM 510 META confocal scanning laser microscope (Carl Zeiss Microimaging, Thornwood, NY). The increase in fluorescence was analyzed in 10-15 individual cells and expressed as the increase in fluorescence before stimulation and after TGF- $\beta 1$ stimulation (20 min) in percentage using MetaMorph (MetaMorph Inc, Nashville, TN). 


\subsection{Western blotting}

Following treatments, cell lysates were prepared on ice by using Western solubilization buffer $\left(50 \mathrm{mmol} / \mathrm{L}\right.$ HEPES, $250 \mathrm{mmol} / \mathrm{L} \mathrm{NaCl}, 1.5 \mathrm{mmol} / \mathrm{L} \mathrm{MgCl}_{2}, 1 \%$ Triton-X100, 10\% glycerol, $1 \mathrm{mmol} / \mathrm{L}$ ethyleneglycol-bis-( $\beta$-aminoethylether)- $N, N, N^{\prime}, N^{\prime}$-tetraacetic acid, $1 \mathrm{mmol} / \mathrm{L}$ phenylmethylsulfonyl fluoride, $2 \mathrm{mmol} / \mathrm{L} \mathrm{Na}_{3} \mathrm{VO}_{4}, 10 \mu \mathrm{g} / \mathrm{mL}$ aprotinin, and $10 \mu \mathrm{g} / \mathrm{mL}$ leupeptin; $\mathrm{pH} 7.4$ ), and cell lysate protein concentration was measured by using BCA protein assay kit (Thermo Scientific). Aliquots of cell lysates (containing $20 \mu \mathrm{g}$ protein) were separated on $10 \%$ or $12 \%$ SDS-PAGE gels (Invitrogen), transferred to nitrocellulose membranes, and probed with antibodies against FYN (1:1000), phosho-SRC family Tyr416 (1:1000), phospho-SRC Tyr527 (1:1000), SRC (L4A1; 1:1000), yH2AX (20E3; 1:1000) and $\beta-$ ACTIN (1:5000; all from Cell Signaling, Danvers, MA). Primary antibodies were probed with rabbit, mouse (Cell Signaling, Danvers, MA) or goat-specific secondary antibodies conjugated with horseradish peroxidase (Thermo Scientific) and detected by means of chemiluminescence with SuperSignal West Pico or Femto Chemiluminescent Substrate (Thermo Scientific). Integrated density of pixels in each membrane was quantified using ImageJ 1.47v (W. Rasband, National Institutes of Health, Bethesda, MD).

\subsection{Analysis of protein cysteine oxidation}

For analysis of protein cysteine oxidation to sulfenic acids (-S-OH), treated cells were lysed in Western solubilization buffer containing $1 \mathrm{mmol} / \mathrm{L}$ of the sulfenic acid-specific probe DCP-Bio1 (Kerafast, Boston, MA), as well as $200 \mathrm{U} / \mathrm{mL}$ catalase (Worthington, Lakewood, $\mathrm{NJ}$ ), and $10 \mathrm{mmol} / \mathrm{L} \mathrm{N}$-ethylmaleimide (Sigma), and incubated for $1 \mathrm{hr}$ at $4^{\circ} \mathrm{C}$ with constant rotation (33). After derivatization, excess biotinylating reagent was removed by 6 washes for $15 \mathrm{~min}$ with $20 \mathrm{mmol} / \mathrm{L}$ Tris- $\mathrm{HCl}(\mathrm{pH} \mathrm{7.4)}$ ) on Amicon Ultra-0.5 Centrifugal Filter Devices (Millipore, Temecula, CAb). Subsequently, biotin-tagged proteins were collected with NeutrAvidin agarose beads ( $50 \mu \mathrm{L}$ of $50 / 50$ solution, Pierce) and washed with $1 \%$ SDS, $4 \mathrm{~mol} / \mathrm{L}$ urea, $1 \mathrm{~mol} / \mathrm{L} \mathrm{NaCl}$, and $100 \mathrm{mmol} / \mathrm{L}$ ammonium bicarbonate (with and without $10 \mathrm{mmol} / \mathrm{L}$ dithiothreitol) to remove nonspecifically bound proteins (34). To elute biotinylated proteins, NeutrAvidin beads were suspended in $6 \times$ reducing sample buffer containing $\beta$-mercaptoethanol and heated for $10 \mathrm{~min}$ at $90^{\circ} \mathrm{C}$. The eluted proteins were analyzed using SDS-PAGE and Western blotting for proteins of interest, and whole cell lysates were analyzed similarly as input controls.

\subsection{Analysis of DNA damage by immunofluorescence staining}

Stimulated cells on chamber slides (Merck Millipore, Billerica, MA) were washed with PBS and fixed with $4 \%$ paraformaldehyde for $30 \mathrm{~min}$, rinsed with PBS and incubated in PBS containing $1 \%$ BSA and $0.2 \%$ Triton X-100 for 15 min. Slides were then blocked with PBS containing $10 \%$ normal goat serum for 60 minutes and stained with antibodies targeted at YH2AX Ser139 (1:400, Cell Signaling) overnight. The next day, Alexa Fluor secondary antibody (Invitrogen) targeted against $\mathrm{yH} 2 \mathrm{AX}$ was added followed by 4'-6-diamidino-2- 
phenylindole dihydrochloride staining (DAPI, Invitrogen). Images were captured with a 40X objective using a Zeiss LSM 510 META confocal scanning laser microscope and quantification of images was performed on a minimum of 200 cells per condition using MetaMorph. Nuclear $\mathrm{\gamma H} 2 \mathrm{AX}$ staining was quantified using DAPI overlay and expressed as integrated intensity divided by the area of the DAPI staining.

\subsection{IPF gene expression data}

Microarray data for NOX4 and FYN were obtained from the Lung Tissue Research Consortium dataset GSE47460 (www.ncbi.nlm.nih.gov/geo), which includes lung tissue RNA samples collected during thoracic surgery. In total, 105 healthy controls and 159 patients diagnosed with IPF by clinical history, CT scan and surgical pathology were included, after removal of outliers by hierarchical clustering. Tissue expression of genes of interest were correlated with available lung function data (diffusion capacity for carbon monoxide (DLCO) expressed as percentage of predicted values based on age and gender). Microarray data were normalized using a cyclic loess approach as previously described (35).

\subsection{Statistical analysis}

All quantitative data are represented as means \pm SEM. Statistical differences between groups were evaluated by 1-way ANOVA analysis with Bonferroni's post-hoc analysis or by Student's t-test, depending on appropriate datasets, using GraphPad Prism software (version 7.3; GraphPad Software, La Jolla, CA), and considered significant at a $P$ value of less than 0.05 . 


\section{Results}

\subsection{TGF- $\beta 1$ induces profibrotic responses by activating the SFK FYN}

We addressed the ability of TGF- $\beta 1$ to induce SFKs in HBE1 epithelial cells and observed that TGF- $\beta 1$ stimulation $(10 \mathrm{ng} / \mathrm{mL}$ ) induced a rapid increase in SFK activity, indicated by (auto)phosphorylation at Tyr416, with a peak around $30 \mathrm{~min}$ followed by a decrease after $2 \mathrm{hrs}$ (Figure 5.1A and B). Activation of SFKs typically involves dephosphorylation at Tyr527 $27^{1}$ (36), but TGF- $\beta 1$ did not significantly alter overall Tyr527 phosphorylation, suggesting that SFK is activated through a non-canonical pathway (Figures 5.1A and B). To evaluate the effect of SFK inhibition on induction of profibrotic genes by TGF- $\beta 1$, cells were stimulated for $24 \mathrm{hrs}$ with TGF- $\beta 1(10 \mathrm{ng} / \mathrm{mL})$ in the absence or presence of the SFK inhibitor PP2 (1 $\mu \mathrm{M})$. As expected, TGF- $\beta 1$ stimulation increased expression of the profibrotic genes COL1A1, VIM and FN1, and each of these responses was reduced in PP2treated cells (Figure 5.1C), indicating that SFKs contribute to TGF- $\beta 1$-mediated profibrotic responses in epithelial cells. Another important feature of TGF- $\beta 1$-induced fibrotic responses is the induction of DNA damage responses $(8,37,38)$. We determined DNA damage response induced by TGF- $\beta 1$ by assessing phosphorylation of the histone variant $\mathrm{H} 2 \mathrm{AX}$, referred to as $\mathrm{\gamma H} 2 \mathrm{AX}$ (Figure 5.1D, Supplemental Figure E1). Pretreatment with the SFK inhibitor PP2 also significantly reduced TGF- $\beta 1$-induced DNA damage response, shown by a decrease of $\gamma \mathrm{H} 2 \mathrm{AX}$ staining (Figure $5.1 \mathrm{E}$ ). Hence, SFKs contribute importantly to TGF- $\beta 1$-induced epithelial responses that promote fibrosis.

The SRC family of protein tyrosine kinases includes nine members, of which SRC, FYN, and YES are ubiquitously expressed (31). All three are also expressed in HBE1 cells (Supplemental Figure E5.2), but their expression was not altered after TGF- $\beta 1$ stimulation (Supplemental Figure E5.3). To determine the involvement of individual SFKs in TGF- $\beta$ mediated profibrotic responses, we used specific small interference RNA to silence them individually. Surprisingly (32), TGF- $\beta 1$-induced SFK activity, measured as phosphorylation of Tyr416, was not affected by knockdown of SRC but was significantly reduced upon silencing FYN (Figures 5.2A-D). Hence, SFK-mediated profibrotic responses to TGF- $\beta 1$ may be primarily mediated by FYN. Accordingly, siRNA silencing of FYN also significantly attenuated the TGF- $\beta$-induced DNA damage response, assessed as $\mathrm{\gamma H} 2 \mathrm{AX}$ (Figures 5.2E and $\mathrm{F})$. 
A

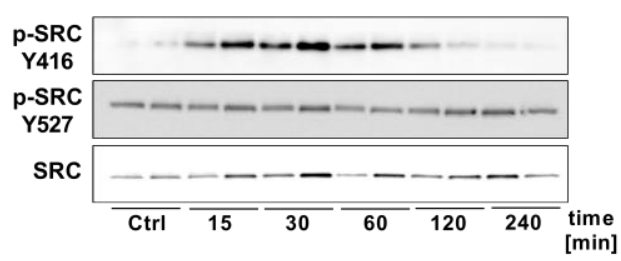

C

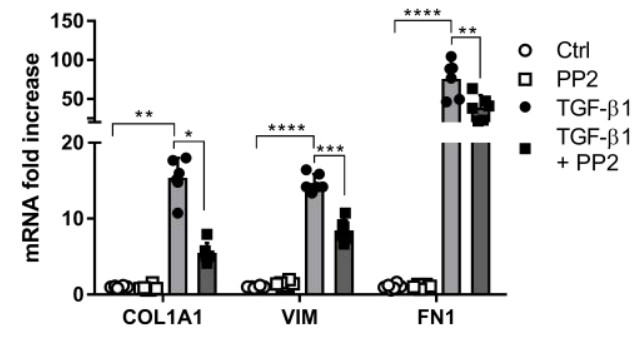

D
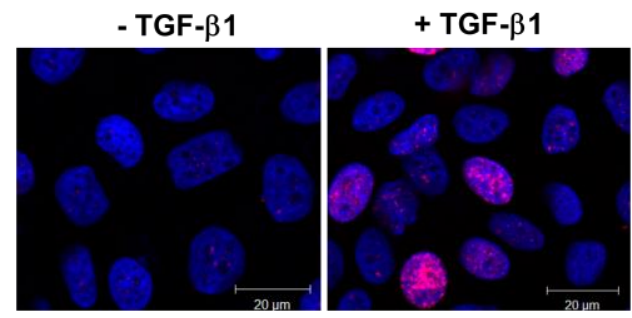

B

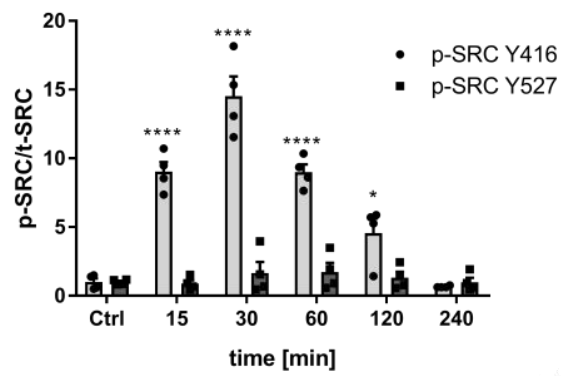

E

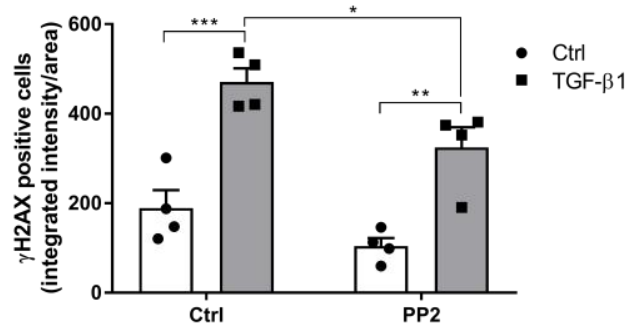

Figure 5.1: TGF- $\beta 1$ induces SFK-mediated profibrotic gene expression and DNA damage response. (A) Representative western blots indicating phosphorylation of SRC kinases at tyrosine 416 and 527 after stimulation with TGF- $\beta 1(10 \mathrm{ng} / \mathrm{mL})$. (B) Densitometry analysis of 4 replicates from 2 independent experiments. (C) Gene expression of profibrotic genes after stimulation with TGF- $\beta 1(10 \mathrm{ng} / \mathrm{mL})$ for $24 \mathrm{hrs}$, in the absence or presence of PP2, determined by RT-PCR ( $n=6)$. (D) Representative immunofluorescence imaging of $\gamma H 2 A X($ red) and 4'-6diamidino-2-phenylindole dihydrochloride (DAPI; blue) in unstimulated or TGF- $\beta 1$-stimulated (4 hrs) HBE1 cells. (E) Quantitative analysis of nuclear $\mathrm{HH} 2 \mathrm{AX}$ intensity in HBE1 cells in the absence or presence of PP2 (1 $\mu \mathrm{M})$ with and without TGF- $\beta 1(10 \mathrm{ng} / \mathrm{mL})$ stimulation for $4 \mathrm{hrs}$. For each experimental condition, a minimum of 200 cells from in total 2 independent experiments with 2 replicates were analyzed. Results are expressed as mean \pm SEM $(* \mathrm{P}<0.05, * * \mathrm{P}<0.01, * * * \mathrm{P}<0.001, * * * * \mathrm{P}<0.0001)$. 
A

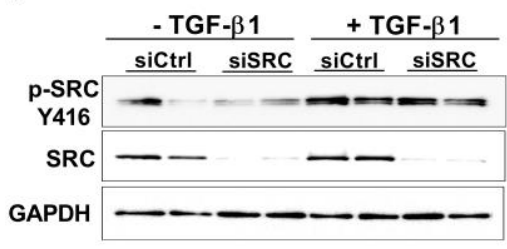

C

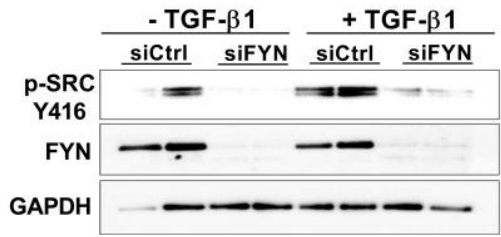

$\mathbf{E}$

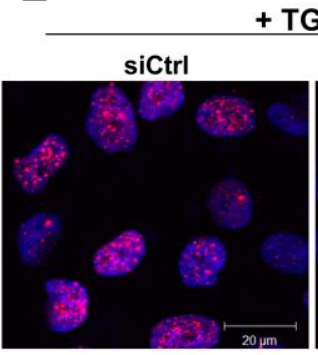

B

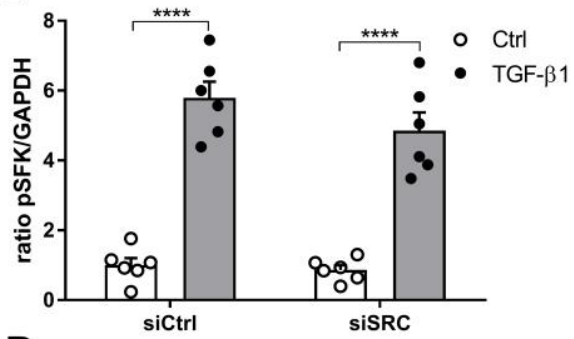

D

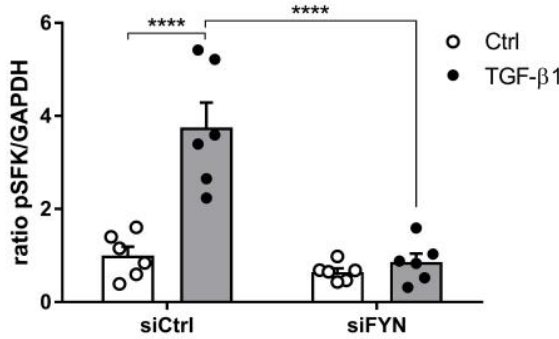

$\mathbf{F}$

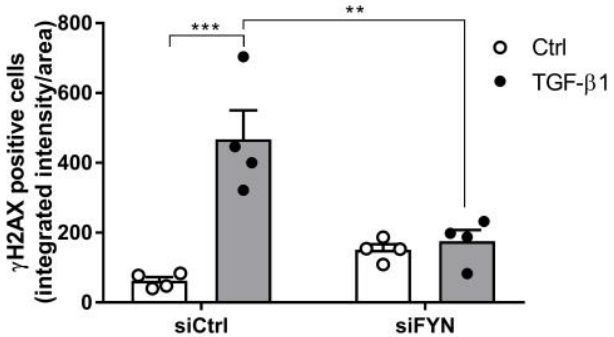

Figure 5.2: TGF- $\beta 1$-induced SFK activation and profibrotic responses depend on FYN. (A) Knockdown of SRC does not reduce phosphorylation at Tyr416 after stimulation with TGF- $\beta 1(10 \mathrm{ng} / \mathrm{mL})$. (B) Densitometry analysis of 3 independent experiments ( $n=6)$. (C) Phosphorylation at Tyr416 is decreased in FYN-silenced cells after 30 min of TGF- $\beta 1(10 \mathrm{ng} / \mathrm{mL})$ stimulation. (D) Densitometry analysis of 3 independent experiments $(n=6)$. (E) Representive image of $\mathrm{YH} 2 \mathrm{AX}$ (red) and DAPI (blue) stained control and FYN-silenced HBE1 cells stimulated with TGF- $\beta 1$ for 4 hrs. (F) Analysis of nuclear $\gamma \mathrm{H} 2 \mathrm{AX}$ intensity in HBE1 cells in Ctrl and FYN-silenced cells with and without TGF- $\beta 1$ stimulation for $4 \mathrm{hrs}$. For each experimental condition, a minimum of 200 cells from in total 2 independent experiments with 2 replicates were analyzed. Results are expressed as mean \pm SEM $\left({ }^{*} P<0.05,{ }^{*} P<0.01\right.$, $* * * \mathrm{P}<0.001, * * * * \mathrm{P}<0.0001)$. 


\subsection{TGF- $\beta 1$ activates FYN by a NOX4-dependent redox mechanism}

SFKs are activated primarily by (de)phosphorylation events, but are also subject to redox regulation through reversible oxidation of conserved cysteines $(31,39)$. To assess redoxbased mechanisms, we first determined whether TGF- $\beta 1$ induced extracellular or intracellular production of ROS in HBE1 cells. Indeed, TGF- $\beta 1$ significantly enhanced intracellular but not extracellular ROS (Figures 5.3A and B). To determine the possible source of TGF- $\beta 1$-induced intracellular ROS production, we assessed potential changes in expression of diverse NOX isoforms. As expected $(12,14,19)$, TGF- $\beta 1$ stimulation of HBE1 cells for $4 \mathrm{hrs}$ induced expression of NOX4 but did not affect expression of other NOX isoforms (Supplemental Figure E5.4). To examine the contribution of NOX4 to FYN activation, it was silenced by siRNA approaches (Supplemental Figure E5.5), which indeed markedly reduced TGF- $\beta 1$-induced (auto)phosphorylation of FYN, compared to controltransfected cells (Figures $5.3 \mathrm{C}$ and $\mathrm{D}$ ). To determine whether such NOX4-mediated FYN activation was associated with cysteine oxidation within FYN, we used a dimedone trapping strategy to detect formation of intermediate sulfenic acids within. Indeed, TGF$\beta 1$ stimulation was found to induce cysteine sulfenylation of FYN, at time points corresponding with its autophosphorylation (Figures 5.3E and F). Moreover, TGF- $\beta 1$ induced sulfenylation of FYN was suppressed in NOX4-silenced cells compared to controls (Figure 5.3G and H), indicating a critical role for NOX4. Collectively, our findings indicate that TGF- $\beta 1$-induced activation of FYN and subsequent profibrotic responses are initiated by NOX4-mediated redox signaling and sulfenylation of FYN. 
A

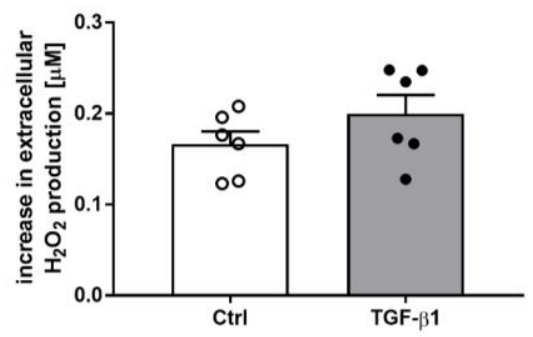

C

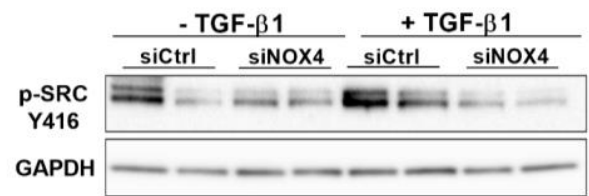

$\mathbf{E}$
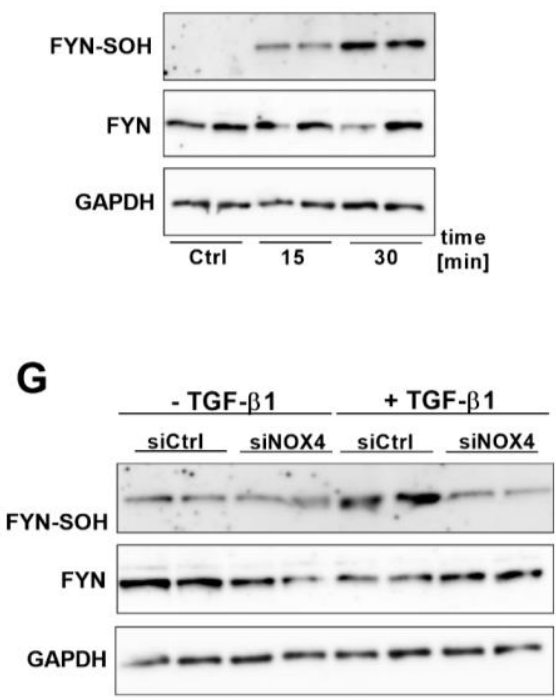

B

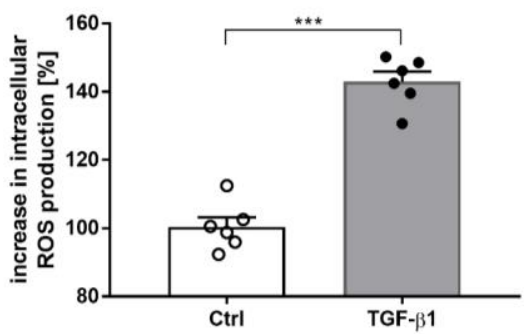

D

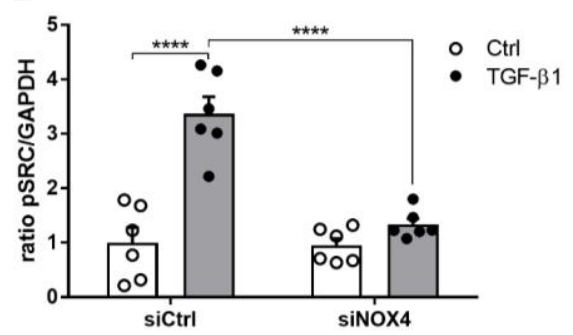

$\mathbf{F}$

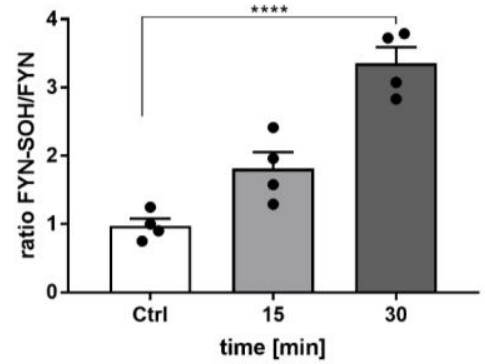

H

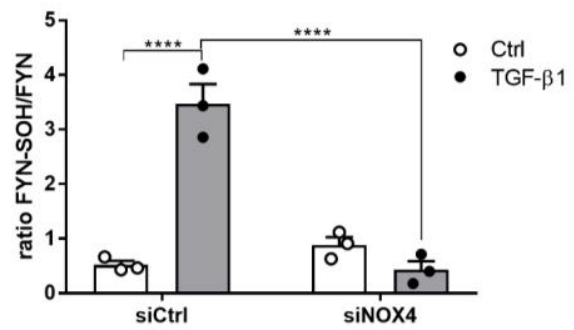

Figure 5.3: TGF- $\beta 1$ activates FYN by NOX4-dependent redox signaling. (A) Effect of TGF- $\beta 1$ (10ng/mL; $30 \mathrm{~min}$ ) on extracellular $\mathrm{H}_{2} \mathrm{O}_{2}$ production, measured using Amplex Red $(n=6)$. (B) Analysis of intracellular ROS production using $\mathrm{H}_{2}$ DCF-DA, in the absence or presence of TGF- $\beta 1$ (10 ng/mL; $\left.30 \mathrm{~min}\right)$. Fluorescence intensity is expressed relative to unstimulated controls ( $n=6)$. (C) Phosphorylation of SFKs (Tyr416) in NOX4 silenced cells compared to controls after $30 \mathrm{~min}$ of stimulation with TGF- $\beta 1(10 \mathrm{ng} / \mathrm{mL})$. (D) Densitometry analysis of 3 independent experiments $(n=6)$. (E) Sulfenylation of FYN and total FYN analyzed by Western blotting. (F) Quantification of sulfenylated cysteine levels of FYN in 3 independent experiments ( $n=6)$. (G) FYN sulfenylation in NOX4 silenced 
cells. (H) Quantification of sulfenylated cysteine levels of FYN in 2 independent experiments $(n=3)$. Results are expressed as mean \pm SEM $\left({ }^{* * *} \mathrm{P}<0.001,{ }^{* * * *} \mathrm{P}<0.0001\right)$.

\subsection{TGF-B1-mediated profibrotic responses involve FYN-mediated mitochondrial ROS production}

A number of studies indicate that IPF development is associated with mitochondrial dysfunction and mitochondrial ROS (mtROS) production $(40,41)$, but it is unclear how mtROS are regulated. To investigate whether TGF- $\beta 1$-induced SFK activation promotes generation of mtROS, we evaluated mtROS formation using live-cell imaging analysis with the mtROS indicator MitoSOX. Indeed, stimulation with TGF- $\beta 1$ markedly enhanced mtROS production within 20 min, which was dramatically attenuated by the SFK inhibitor PP2 (Figures 5.4A and B). Similarly, TGF- $\beta 1$-induced mtROS production was also attenuated after silencing of FYN (Figure 5.4C, Supplemental Figure E5.6). We next determined whether mtROS production was involved in TGF- $\beta 1$-induced profibrotic responses, by cell pretreatment with the mitochondria-targeted antioxidant MitoQ. Indeed, MitoQ pretreatment attenuated TGF- $\beta 1$-induced expression of the profibrotic gene FN1 (Figure 5.4D) as well as the presence of $\mathrm{\gamma H} 2 \mathrm{AX}$ foci (Figures 5.4E). MitoQ did not affect TGF- $\beta 1$-induced SFK activation (Supplemental Figure E5.7), consistent with the notion that SFKs are involved in the upstream regulation of mtROS production. Collectively, these results indicate that NOX4-induced activation of the SRC kinase FYN is required to facilitate TGF- $\beta 1$-induced mtROS production, which subsequently contributes to profibrotic responses. 
A

unstimulated

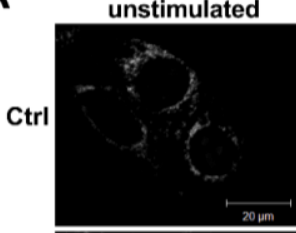

P2

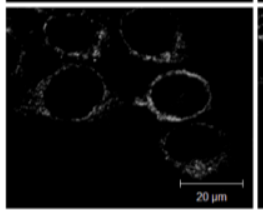

C

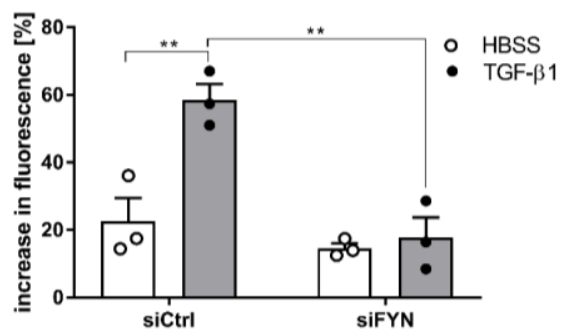

$\mathbf{E}$

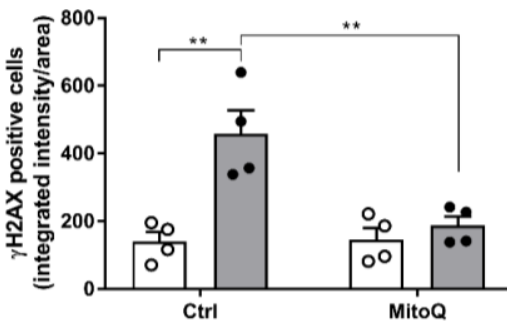

- Ctrl

- TGF- $\beta 1$
B

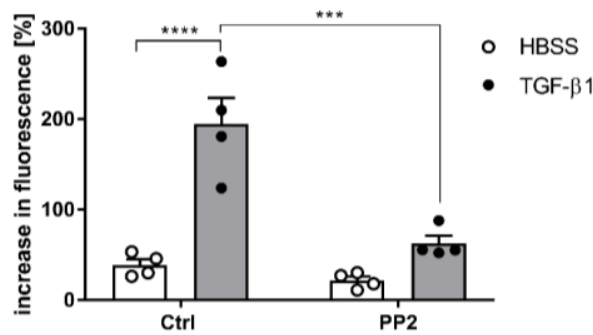

D

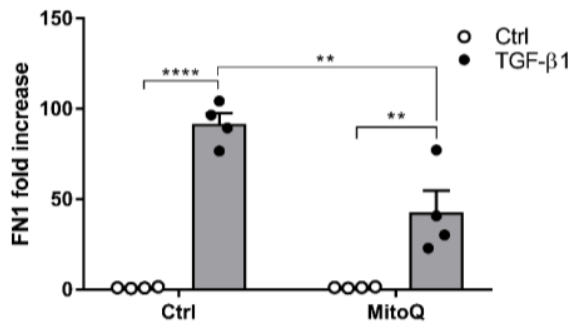

Figure 5.4: Mitochondrial ROS production mediates TGF- $\beta 1$-mediated fibrotic responses. (A) Representative image of mtROS analysis using MitoSOX in unstimulated or TGF- $\beta 1$-stimulated $(10 \mathrm{ng} / \mathrm{mL} ; 20 \mathrm{~min}) \mathrm{HBE} 1 \mathrm{cells}$. (B) Quantification of MitoSOX fluorescence increase over 20 minutes following TGF- $\beta 1$ stimulation in the absence or presence of PP2 $(1 \mu \mathrm{M})$. Fluorescence increase was quantified in 10-15 individual cells from 3 independent experiments $(n=3)$ and expressed as mean \pm SEM. (C) Quantification of TGF- $\beta 1$-stimulated MitoSOX fluorescence increase in siCtrl and siFYN cells. Increase in fluorescence was analyzed in 10-15 individual cells from 4 independent experiments ( $n=4)$, and expressed as mean \pm SEM. (D) FN1 expression after 24 hrs stimulation with TGF- $\beta 1$ with or without pretreatment with the mitochondrial antioxidant MitoQ $(n=4)$. Results are expressed as mean \pm SEM. (E) Analysis of nuclear $\gamma \mathrm{H} 2 \mathrm{AX}$ immunofluorescence in TGF- $\beta 1$-stimulated HBE1 cells (4 hrs) pretreated with MitoQ. For each experimental condition, a minimum of 200 cells from in total 2 independent experiments with 2 replicates were analyzed. Results are expressed as mean \pm SEM $(* * P<0.01, * * * P<0.001$, $* * * * P<0.0001$ ). 


\subsection{Gene expression of NOX4 and FYN is altered in IPF in association with disease severity}

To assess the importance of NOX4 and FYN in human IPF, we examined their expression profiles in lung tissues from patients with IPF available through the Lung Tissue Research Consortium. Somewhat surprisingly, NOX4 gene expression was not significantly altered between control subjects and IPF patients (Figure 5.5A), although NOX4 expression levels correlated inversely with the predicted diffusion capacity for carbon monoxide (DLCO) indicating that NOX4 expression is associated with disease severity (Figure 5.5B). Interestingly, lung tissue expression FYN was found to be significantly decreased in patients with IPF (Figure 5.5C), although FYN expression levels did not correlate with disease severity (Figure 5.5D). These observations suggest that dysregulation NOX4/FYN expression in IPF may lead to altered regulation of these signaling pathways and contribute to disease severity.

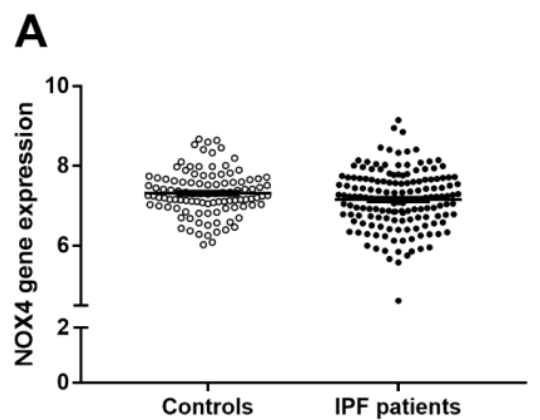

C

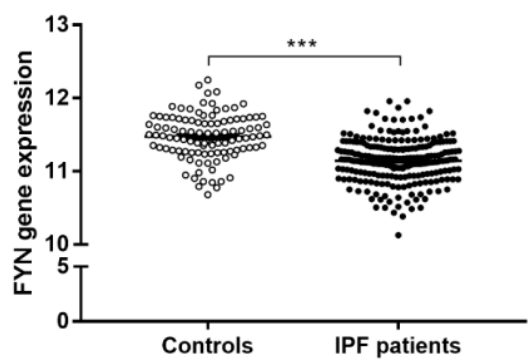

B

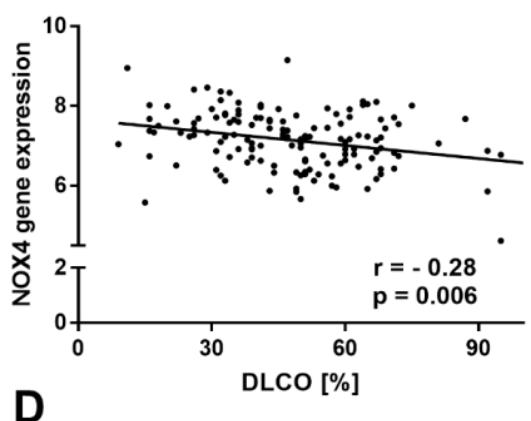

D

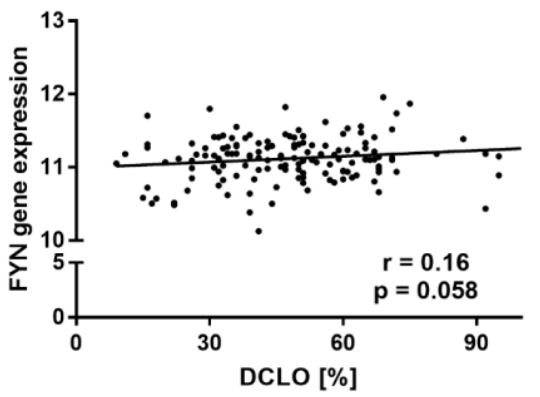

Figure 5.5: NOX4 and FYN mRNA expression in controls ( $n=105)$ and IPF patients ( $n=159)$. (A) NOX4 expression levels in lung tissues from IPF patients and controls. (B) Correlation of NOX4 expression with DLCO (\%). (C) FYN expression in lungs of control subject and IPF patients. (D) Correlation of FYN expression with DLCO (\%). Results are expressed as mean \pm SD. Differences between IPF patients and controls were determined by student's t-test and correlation between NOX4/FYN and DLCO was analyzed by Pearson's correlation (***P<0.001). 


\section{Discussion}

The present studies addressed the mechanisms by which the growth factor TGF- $\beta 1$ induces fibrotic responses within the lung epithelium and demonstrate that TGF- $\beta 1$ induces both DNA damage responses and profibrotic gene expression in human epithelial cells. Both features were found to be mediated by NOX4-dependent activation of the SRC kinase FYN, which in turn causes increased production of mtROS and subsequent profibrotic responses. Recent studies have implicated SRC kinases in the pathophysiology of IPF, as they are capable of promoting myofibroblast differentiation and inducing features of $\operatorname{EMT}(21,22)$. However, these observations were largely based on pharmacological SFK inhibitors such as saracatinib or dasatinib, which lack isoform specificity and also inhibit other tyrosine kinases. TGF- $\beta 1$ also induces SFK activation in lung epithelial cells (32), and our studies indicate that this primarily involves the FYN kinase rather than SRC.

Activation of SFKs is regulated by dephosphorylation of Tyr527 and autophosphorylation at Tyr416 within the kinase domain, which help unclamp the kinase and render the protein fully active (36). Additionally, redox-based mechanisms can also contribute to the activation of SFK due to oxidation of one or more conserved cysteine residues within its SH2 or kinase domains $(32,39,42,43)$. Indeed, our observation that TGF- $\beta 1$ enhances SFK phosphorylation at Tyr416 without significant dephosphorylation at Tyr527 suggests an alternative redox-dependent mechanism, as suggested previously (32). Furhermore, activation of FYN by TGF- $\beta 1$ was temporally associated with sulfenylation of one or more of its cysteine residues, and both were found to be mediated by initial activation of NOX4. We recently reported that redox-dependent activation of SRC primarily involves sulfenylation of two cysteine residues within SRC (Cys185 and Cys277) (42). Curiously, these cysteines are not present within FYN, which indicates that oxidation of alternative cysteines must be mediating FYN activation. The most likely candidate is Cys488 as it is conserved among all SFKs and is homologous to C487 in SRC, which has been implicated in its redox-dependent activation (39). More detailed future studies will be needed to clarify the significance of FYN sulfenylation for regulating its activity. NOX4 has been strongly implicated in IPF pathology, due to its involvement in fibroblast differentiation and myofibroblast proliferation $(12,14)$. Moreover, NOX4 was also found to contribute to bleomycin-induced alveolar epithelial cell death in mice, thus representing an alternative mechanism by which NOX4 activation promotes pulmonary fibrosis (15). Indeed, NOX4deficient mice are protected from developing bleomycin-induced pulmonary fibrosis indicating a key role for NOX4 in the development of IPF $(12,44)$. Our findings indicate that NOX4 may promote fibrosis, at least in part, by redox-dependent activation of FYN.

Our observations implicating NOX4 in acute cell responses to TGF- $\beta 1$, such as activation of FYN, would suggest that TGF- $\beta 1$ causes activation of NOX4, which contrasts with the 
common notion that NOX4 function is primarily regulated transcriptionally and is constitutively active (45). Indeed, we observed increased NOX4 mRNA at 4 hrs after TGF$\beta 1$ but not within the time-scale required for FYN activation (15-30 $\mathrm{min}$ ). An intriguing recent study indicated that NOX4 is subject to negative allosteric regulation by ATP (46) and its activity could therefore be enhanced by localized ATP degradation in response to TGF- $\beta$. Induction of NOX4 expression during chronic TGF- $\beta 1$ stimulation or in IPF, combined with metabolic alterations and ATP degradation (47), could therefore lead to augmented NOX4 activation and enhanced disease pathology.

Age-related lung diseases such as IPF are also characterized by mitochondrial dysfunction and enhanced mtROS production, which contributes to epithelial cell death as well as profibrotic gene expression $(48,49)$. Here, we demonstrate that both NOX4 and FYN are required for TGF- $\beta 1$-mediated mtROS production. Intriguingly, both NOX4 (11) and FYN may be present in mitochondria, as it has been shown before that NOX4 and FYN were found to colocalize in mitochondria of e.g. cardiomyocytes (50). It has been reported that NOX4 is able to interact with mitochondrial complex I, thereby negatively regulating its activity (51). Overexpression of NOX4 might have a role in mediating mitochondrial dysfunction in lung fibroblasts (52). SRC family kinases are also recognized as important metabolic regulators in mitochondria (53). For example, mitochondrial localized FYN can promote phosphorylation of complex I, II and IV subunits as well as pyruvate dehydrogenase, thereby regulating their function $(54,55)$. Moreover, FYN is also involved in the regulation of mitochondrial protein synthesis and oxidative phosphorylation indicating its importance in regulating mitochondrial function as well as mtROS production (56). Our findings indicate that TGF- $\beta 1$-induced production of mtROS is important for induction of DNA damage and profibrotic cytokine expression. In addition, TGF- $\beta 1$ signaling also results in induction of NOX4 expression, thus resulting in amplified redox alterations and profibrotic responses in the context of chronic TGF- $\beta 1$ activation as seen in IPF.

In conclusion, we demonstrate that TGF- $\beta 1$-induced fibrotic responses in lung epithelial cells are mediated by NOX4-induced activation of the SRC kinase FYN, which in turn induces mtROS production and epithelial DNA damage responses as well as induction of profibrotic genes. It is important to note that our studies concentrated on acute TGF- $\beta 1$ signaling in epithelial cells, whereas biological effects of TGF- $\beta$ may be altered in chronic conditions such as IPF. As discussed above, this involves induction of NOX4 expression and mitochondrial dysfunction, but could also involve altered expression or activation of FYN. With respect to the latter, it is interesting to note that FYN can negatively regulate NOX4 activity by phosphorylating Tyr566 in its C-terminus (50). Hence, NOX4 and FYN show a reciprocal relationship, with NOX4 being able to activate FYN, whereas FYN can in turn inhibit NOX4 through a negative feedback mechanism. Our findings indicating significant downregulation of FYN in IPF lung tissues may suggest that such negative feedback is 158 
impaired in the context of IPF, thereby allowing for prolonged or enhanced NOX4 activation and downstream profibrotic effects. The fact that NOX4 expression correlates with disease severity in IPF patients, even though its overall expression levels were not enhanced in IPF lungs compared to controls, may be related to such impaired FYNmediated regulation of NOX4 function. In this case, inhibition of FYN by SFK inhibitors may not necessarily be beneficial in IPF as it could potentially amplify NOX4 function and enhance NOX4-mediated profibrotic phenomena independent of FYN. 


\section{Acknowledgements}

AvdV gratefully acknowledges grant support from the National Institute of Health (R01 HL085646, R01 HL138708, and R21 AG055325). CV is supported by the Nutrim Graduate Programme (Maastricht University). The authors also thank Nicole Bishop for assistance with confocal microscopy imaging, which was performed on a Zeiss 510 META laser scanning confocal microscope supported by NIH Award Number 1S10RR019246 from the National Center for Research Resources. 


\section{References}

1. Raghu G, Collard HR, Egan JJ, Martinez FJ, Behr J, Brown KK, et al. An official ATS/ERS/JRS/ALAT statement: idiopathic pulmonary fibrosis: evidence-based guidelines for diagnosis and management. Am J Respir Crit Care Med. 2011;183(6):788-824.

2. Xu X, Dai H, Wang C. Epithelium-dependent profibrotic milieu in the pathogenesis of idiopathic pulmonary fibrosis: current status and future directions. The clinical respiratory journal. 2016;10(2):133-41.

3. Raghu G, Selman M. Nintedanib and pirfenidone. New antifibrotic treatments indicated for idiopathic pulmonary fibrosis offer hopes and raises questions. Am J Respir Crit Care Med. 2015;191(3):252-4.

4. Kuwano K, Nakashima N, Inoshima I, Hagimoto N, Fujita M, Yoshimi M, et al. Oxidative stress in lung epithelial cells from patients with idiopathic interstitial pneumonias. The European respiratory journal. 2003;21(2):232-40.

5. Liepelt A, Tacke F. Healing the scars of life-targeting redox imbalance in fibrotic disorders of the elderly. Ann Transl Med. 2015;3(Suppl 1):S13.

6. Kliment CR, Oury TD. Oxidative stress, extracellular matrix targets, and idiopathic pulmonary fibrosis. Free Radic Biol Med. 2010;49(5):707-17.

7. Psathakis K, Mermigkis D, Papatheodorou G, Loukides S, Panagou P, Polychronopoulos V, et al. Exhaled markers of oxidative stress in idiopathic pulmonary fibrosis. Eur J Clin Invest. 2006;36(5):362-7.

8. Povedano JM, Martinez P, Flores JM, Mulero F, Blasco MA. Mice with Pulmonary Fibrosis Driven by Telomere Dysfunction. Cell Rep. 2015;12(2):286-99.

9. Martinez FJ, de Andrade JA, Anstrom KJ, King TE, Jr., Raghu G. Randomized trial of acetylcysteine in idiopathic pulmonary fibrosis. The New England journal of medicine. 2014;370(22):2093-101.

10. Anathy V, Lahue KG, Chapman DG, Chia SB, Casey DT, Aboushousha R, et al. Reducing protein oxidation reverses lung fibrosis. Nat Med. 2018;24(8):1128-35.

11. Bernard K, Hecker L, Luckhardt TR, Cheng G, Thannickal VJ. NADPH oxidases in lung health and disease. Antioxidants \& redox signaling. 2014;20(17):2838-53.

12. Hecker L, Vittal R, Jones T, Jagirdar R, Luckhardt TR, Horowitz JC, et al. NADPH oxidase-4 mediates myofibroblast activation and fibrogenic responses to lung injury. Nat Med. 2009;15(9):1077-81.

13. Crestani B, Besnard V, Boczkowski J. Signalling pathways from NADPH oxidase-4 to idiopathic pulmonary fibrosis. Int J Biochem Cell Biol. 2011;43(8):1086-9.

14. Amara N, Goven D, Prost F, Muloway R, Crestani B, Boczkowski J. NOX4/NADPH oxidase expression is increased in pulmonary fibroblasts from patients with idiopathic pulmonary fibrosis and mediates TGFbeta1-induced fibroblast differentiation into myofibroblasts. Thorax. 2010;65(8):733-8.

15. Carnesecchi S, Deffert C, Donati Y, Basset O, Hinz B, Preynat-Seauve O, et al. A key role for NOX4 in epithelial cell death during development of lung fibrosis. Antioxid Redox Signal. 2011;15(3):607-19.

16. Hecker L, Logsdon NJ, Kurundkar D, Kurundkar A, Bernard K, Hock T, et al. Reversal of persistent fibrosis in aging by targeting Nox4-Nrf2 redox imbalance. Sci Transl Med. 2014;6(231):231ra47.

17. Fernandez IE, Eickelberg $\mathrm{O}$. The impact of TGF-beta on lung fibrosis: from targeting to biomarkers. Proceedings of the American Thoracic Society. 2012;9(3):111-6.

18. Wolters PJ, Collard HR, Jones KD. Pathogenesis of idiopathic pulmonary fibrosis. Annu Rev Pathol. 2014;9:157-79. 
19. Boudreau HE, Casterline BW, Rada B, Korzeniowska A, Leto TL. Nox4 involvement in TGFbeta and SMAD3-driven induction of the epithelial-to-mesenchymal transition and migration of breast epithelial cells. Free Radic Biol Med. 2012;53(7):1489-99.

20. Liu RM, Desai LP. Reciprocal regulation of TGF-beta and reactive oxygen species: A perverse cycle for fibrosis. Redox Biol. 2015;6:565-77.

21. Hu M, Che P, Han X, Cai GQ, Liu G, Antony V, et al. Therapeutic targeting of SRC kinase in myofibroblast differentiation and pulmonary fibrosis. The Journal of pharmacology and experimental therapeutics. 2014;351(1):87-95.

22. Abdalla M, Thompson L, Gurley E, Burke S, Ujjin J, Newsome R, et al. Dasatinib inhibits TGFbeta-induced myofibroblast differentiation through Src-SRF Pathway. Eur J Pharmacol. 2015;769:134-42.

23. Roskoski R, Jr. Src protein-tyrosine kinase structure, mechanism, and small molecule inhibitors. Pharmacol Res. 2015;94:9-25.

24. Thomas SM, Brugge JS. Cellular functions regulated by Src family kinases. Annu Rev Cell Dev Biol. 1997;13:513-609.

25. Grimminger F, Gunther A, Vancheri C. The role of tyrosine kinases in the pathogenesis of idiopathic pulmonary fibrosis. Eur Respir J. 2015;45(5):1426-33.

26. Xie YG, Yu Y, Hou LK, Wang X, Zhang B, Cao XC. FYN promotes breast cancer progression through epithelial-mesenchymal transition. Oncol Rep. 2016;36(2):1000-6.

27. Cicchini C, Laudadio I, Citarella F, Corazzari M, Steindler C, Conigliaro A, et al. TGFbetainduced EMT requires focal adhesion kinase (FAK) signaling. Exp Cell Res. 2008;314(1):143-52.

28. Lu YY, Zhao XK, Yu L, Qi F, Zhai B, Gao CQ, et al. Interaction of Src and Alpha-V Integrin Regulates Fibroblast Migration and Modulates Lung Fibrosis in A Preclinical Model of Lung Fibrosis. Sci Rep. 2017;7:46357.

29. Harrison SC. Variation on an Src-like theme. Cell. 2003;112(6):737-40.

30. Roskoski R, Jr. Src protein-tyrosine kinase structure and regulation. Biochem Biophys Res Commun. 2004;324(4):1155-64.

31. Giannoni E, Taddei ML, Chiarugi P. Src redox regulation: again in the front line. Free Radic Biol Med. 2010;49(4):516-27.

32. Zhang H, Davies KJ, Forman HJ. TGFbeta1 rapidly activates Src through a non-canonical redox signaling mechanism. Arch Biochem Biophys. 2015;568:1-7.

33. Hristova M, Habibovic A, Veith C, Janssen-Heininger YM, Dixon AE, Geiszt $M$, et al. Airway epithelial dual oxidase 1 mediates allergen-induced IL-33 secretion and activation of type 2 immune responses. J Allergy Clin Immunol. 2016;137(5):1545-56 e11.

34. Nelson KJ, Klomsiri C, Codreanu SG, Soito L, Liebler DC, Rogers LC, et al. Use of dimedonebased chemical probes for sulfenic acid detection methods to visualize and identify labeled proteins. Methods Enzymol. 2010;473:95-115.

35. McDonough JE, Kaminski N, Thienpont B, Hogg JC, Vanaudenaerde BM, Wuyts WA. Gene correlation network analysis to identify regulatory factors in idiopathic pulmonary fibrosis. Thorax. 2018.

36. Boggon TJ, Eck MJ. Structure and regulation of Src family kinases. Oncogene. 2004;23(48):7918-27.

37. Thannickal VJ, Horowitz JC. Evolving concepts of apoptosis in idiopathic pulmonary fibrosis. Proc Am Thorac Soc. 2006;3(4):350-6.

38. Naikawadi RP, Disayabutr S, Mallavia B, Donne ML, Green G, La JL, et al. Telomere dysfunction in alveolar epithelial cells causes lung remodeling and fibrosis. JCI Insight. 2016;1(14):e86704.

39. Giannoni E, Buricchi F, Raugei G, Ramponi G, Chiarugi P. Intracellular reactive oxygen species activate Src tyrosine kinase during cell adhesion and anchorage-dependent cell growth. Mol Cell Biol. 2005;25(15):6391-403. 
40. Kim SJ, Cheresh P, Jablonski RP, Williams DB, Kamp DW. The Role of Mitochondrial DNA in Mediating Alveolar Epithelial Cell Apoptosis and Pulmonary Fibrosis. Int J Mol Sci. 2015;16(9):21486519.

41. Mora AL, Bueno M, Rojas M. Mitochondria in the spotlight of aging and idiopathic pulmonary fibrosis. J Clin Invest. 2017;127(2):405-14.

42. Heppner DE, Dustin CM, Liao C, Hristova M, Veith C, Little AC, et al. Direct cysteine sulfenylation drives activation of the Src kinase. Nat Commun. 2018;9(1):4522.

43. Yoo SK, Starnes TW, Deng $Q$, Huttenlocher A. Lyn is a redox sensor that mediates leukocyte wound attraction in vivo. Nature. 2011;480(7375):109-12.

44. Jarman ER, Khambata VS, Cope C, Jones P, Roger J, Ye LY, et al. An inhibitor of NADPH oxidase- 4 attenuates established pulmonary fibrosis in a rodent disease model. American journal of respiratory cell and molecular biology. 2014;50(1):158-69.

45. Martyn KD, Frederick LM, von Loehneysen K, Dinauer MC, Knaus UG. Functional analysis of Nox4 reveals unique characteristics compared to other NADPH oxidases. Cell Signal. 2006;18(1):6982.

46. Shanmugasundaram K, Nayak BK, Friedrichs WE, Kaushik D, Rodriguez R, Block K. NOX4 functions as a mitochondrial energetic sensor coupling cancer metabolic reprogramming to drug resistance. Nat Commun. 2017;8(1):997.

47. Kang YP, Lee SB, Lee JM, Kim HM, Hong JY, Lee WJ, et al. Metabolic Profiling Regarding Pathogenesis of Idiopathic Pulmonary Fibrosis. J Proteome Res. 2016;15(5):1717-24.

48. Patel AS, Song JW, Chu SG, Mizumura K, Osorio JC, Shi Y, et al. Epithelial cell mitochondrial dysfunction and PINK1 are induced by transforming growth factor-beta1 in pulmonary fibrosis. PloS one. 2015;10(3):e0121246.

49. Jain M, Rivera S, Monclus EA, Synenki L, Zirk A, Eisenbart J, et al. Mitochondrial reactive oxygen species regulate transforming growth factor-beta signaling. J Biol Chem. 2013;288(2):770-7. 50. Matsushima S, Kuroda J, Zhai P, Liu T, Ikeda S, Nagarajan N, et al. Tyrosine kinase FYN negatively regulates NOX4 in cardiac remodeling. The Journal of clinical investigation. 2016;126(9):3403-16.

51. Koziel R, Pircher H, Kratochwil M, Lener B, Hermann M, Dencher NA, et al. Mitochondrial respiratory chain complex I is inactivated by NADPH oxidase Nox4. Biochem J. 2013;452(2):231-9.

52. Bernard K, Logsdon NJ, Miguel V, Benavides GA, Zhang J, Carter AB, et al. NADPH Oxidase 4 (Nox4) Suppresses Mitochondrial Biogenesis and Bioenergetics in Lung Fibroblasts via a Nuclear Factor Erythroid-derived 2-like 2 (Nrf2)-dependent Pathway. J Biol Chem. 2017;292(7):3029-38. 53. Ogura M, Yamaki J, Homma MK, Homma Y. Mitochondrial c-Src regulates cell survival through phosphorylation of respiratory chain components. The Biochemical journal. 2012;447(2):281-9.

54. Salvi M, Brunati AM, Toninello A. Tyrosine phosphorylation in mitochondria: a new frontier in mitochondrial signaling. Free Radic Biol Med. 2005;38(10):1267-77.

55. Salvi M. Receptor tyrosine kinases take a direct route to mitochondria: an overview. Curr Protein Pept Sci. 2013;14(7):635-40.

56. Koc EC, Miller-Lee JL, Koc H. Fyn kinase regulates translation in mammalian mitochondria. Biochim Biophys Acta. 2017;1861(3):533-40. 


\section{Supplemental Figures}

Figure E5.1: $\underline{T}$ GF- $\beta 1$ induces $\gamma \mathrm{H} 2 \mathrm{AX}$. H2AX phosphorylation at Ser139 after TGF- $\beta 1$ (10 $\mathrm{ng} / \mathrm{mL}$ ) stimulation for various time points determined by Western blotting.

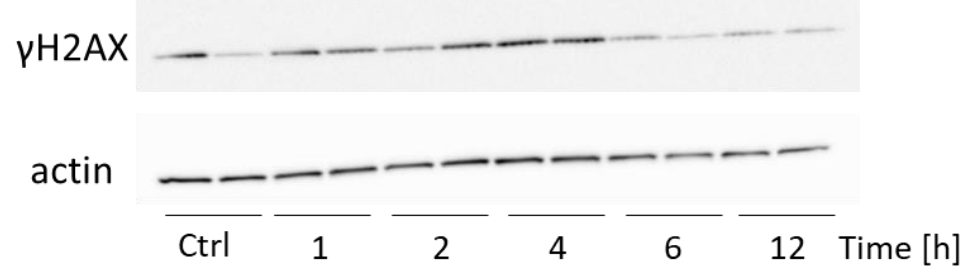

Figure E5.2: Analysis of SFK mRNA expression in HBE1 cells. Gene expression of $c-S R C, F Y N$ and YES analyzed by qRT-PCT $(n=4)$. Results are expressed as mean \pm SEM.

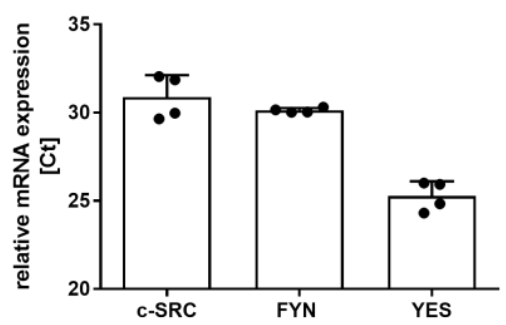

Figure E5.3: TGF- $\beta 1$ does not affect SFK transcriptionally. Gene expression the SRC kinases $c-S R C, F Y N$ and YES relative to GAPDH after stimulation with TGF- $\beta 1(10 \mathrm{ng} / \mathrm{mL})$ for $4 \mathrm{~h}$ analyzed by qRT-PCR $(n=4)$. Results are expressed as mean \pm SEM.

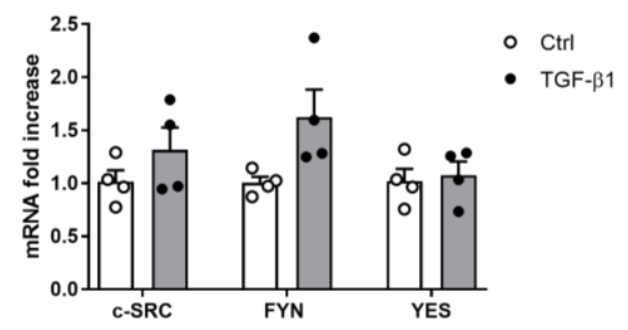


Figure E5.4: Effect of TGF- 31 stimulation on NOX gene expression in HBE1 cells. Gene expression of NOX1, NOX2, NOX4, DUOX1 and DUOX2 relative to GAPDH after stimulation with TGF- $\beta 1(10 \mathrm{ng} / \mathrm{mL})$ for $4 \mathrm{~h}(\mathrm{n}=4)$. Results are expressed as mean $\pm \mathrm{SEM}\left({ }^{*} \mathrm{P}<0.05\right)$.

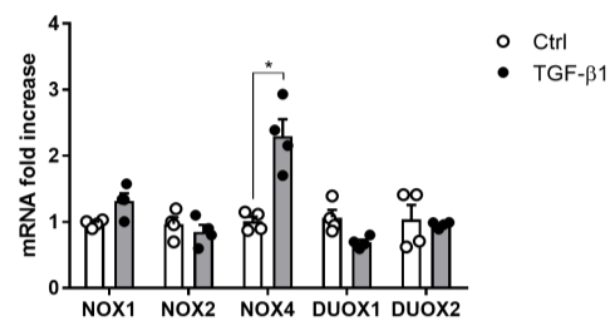

Figure E5.5: Evaluation of siRNA-mediated silencing of NOX4 mRNA in HBE1 cells. Gene expression of NOX4 relative to GAPDH in HBE1 cells transfected with siCtrl or siNOX4, determined using $q R T-P C R(n=4)$. Results are expressed as mean \pm SEM $(* * * P<0.001)$.

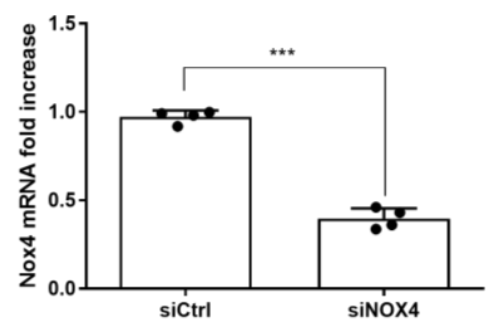


Figure E5.6: TGF- $\beta 1$ induced mitochondrial ROS production is dependent on FYN. Representative image of mtROS analysis using MitoSOX in TGF- $\beta 1$-stimulated $(10 \mathrm{ng} / \mathrm{mL}$; 20 min) control or FYN transfected HBE1 cells.

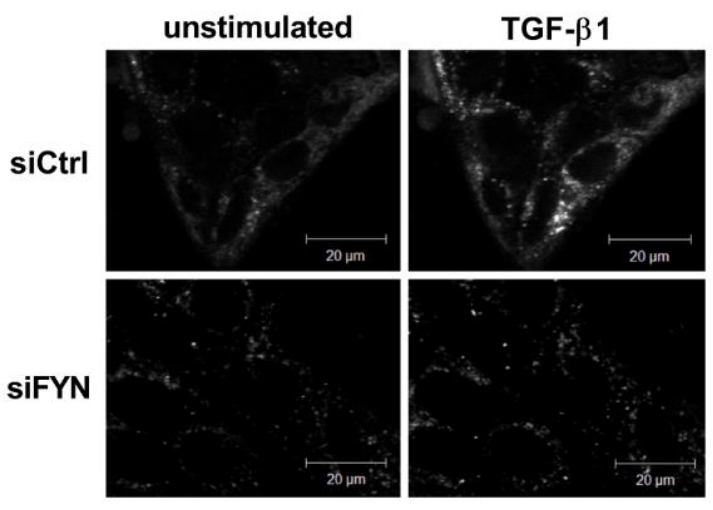

Figure E5.7: TGF- $\beta 1$-mediated SFK activation does not require mitochondrial ROS. SFK phosphorylation at Tyr416 after TGF- $\beta 1(10 \mathrm{ng} / \mathrm{mL})$ stimulation for $30 \mathrm{~min}$ with or without pretreatment with MitoQ $(1 \mu \mathrm{M})$.

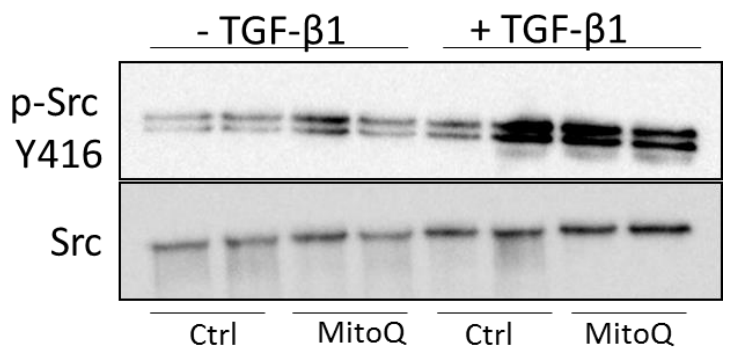






\section{Chapter 6}

SRC family kinases are mediating fibroblast activation through TGF- $\beta 1$ induced activation of NOX4

Carmen Veith, Musa Idris, Marie-José Drittij, Albert van der Vliet, Frederik-Jan van Schooten and Agnes W. Boots.

In preparation 


\section{Abstract}

Idiopathic pulmonary fibrosis (IPF) is a progressive age-related lung disease with a median survival of only 2-4 years after diagnosis. The pathogenic mechanisms behind IPF are still not completely understood and current therapeutic approaches have not been successful in improving disease outcomes. IPF is characterized by continuous injury to the alveolar epithelium leading to overactivation of repair pathways causing excessive collagen deposition by myofibroblasts. Recently, the inhibition of SRC family kinases (SFK) have been suggested as treatment strategy and general inhibitors of SFK reduce profibrotic outcomes in vitro and in vivo. SFKs are involved in various signaling pathways important for cellular homeostasis, which are often dysregulated in lung diseases and become activated in response to transforming growth factor- $\beta$ (TGF- $\beta$ ). However, it is still not clear how SFK are activated and contribute to fibroblast activation and which member of the SRC family is involved. Here, we demonstrate that SFK are activated in response to TGF- $\beta$ and inhibition by the general SFK AZD reduces fibroblast proliferation and activation. Silencing of the SFK member YES reduces expression of the matrix genes fibronectin and collagen as well as expression of NADPH oxidase (NOX) 4. Consequently, our findings suggest the SFK member YES is promoting fibroblast activation potentially through the upregulation of NOX4.

Keywords: IPF, fibroblasts, SRC family kinases, YES, NOX4 


\section{Introduction}

Idiopathic pulmonary fibrosis (IPF) is the end stage of several diffuse parenchymal lung diseases and defined as a chronic fibrotic lung-limited disease primarily occurring in the alveoli and their surrounding tissue (1). The pathogenic mechanisms of IPF are still largely unclear, but over the last decade the paradigm of IPF pathogenesis has shifted from a generally inflammation-driven disease to an epithelial-fibroblastic one $(2,3)$. Common features include continuous epithelial injury by various triggers resulting in aberrant wound healing responses and eventually causing excessive collagen deposition in the alveolar epithelium by myofibroblasts (2). The differentiation of fibroblasts into myofibroblasts is essential in normal wound healing but also promotes fibrotic lesions during abnormal fibrotic repair (4). Key hallmarks of the differentiation of fibroblasts into myofibroblasts include increased proliferative capacity as well as enhanced generation and secretion of extracellular matrix proteins including collagen and fibronectin (5). In addition to remodeling of the lung structure, fibroblasts participate in the regulation of inflammation through the secretion of pro-inflammatory cytokines (6).

Intriguingly, oxidative stress, e.g. the overproduction of oxidants compared to antioxidants, has been suggested to promote a fibrotic milieu in the lung (7). The main cellular sources of reactive oxygen species (ROS) are NADPH oxidases (NOX) and mitochondria (8). Upregulation of NOX4, one of the pulmonary members of the NOX family has been shown in the lungs of IPF patients (9) and, upon activation by transforming-growth-factor (TGF)- $\beta 1$ (10), promotes (myo)fibroblast activation leading to collagen deposition $(9,11)$. Inhibition of NOX4/NOX1 has shown promising results in mouse models of pulmonary fibrosis, but it is difficult to specifically target NOX4-induced ROS without disturbing physiological ROS signaling in patients with IPF.

Treatment strategies targeting the occurring oxidative stress in IPF have not been completely successful until now. Recently, SRC family kinases (SFK) have been suggested as possible treatment targets as they are activated in response to TGF- $\beta$ and important players in the development of lung fibrosis (12-14). SFK are a family of non-receptor tyrosine kinases with nine known members, namely SRC, YES, FYN, FGR, LCK, HCK, BLK, FRK and LYN. SFK are important in cell homeostasis and regulate a spectrum of cellular activities such as cell growth, migration and differentiation (15-17). The activity of SFK is regulated through their phosphorylation status $(18,19)$, but can also through redoxdependent mechanisms $(18,20)$ and in response to TGF- $\beta(12,14)$. Intriguingly, inhibition of SRC kinases by AZD0530 in an experimental model of pulmonary fibrosis reduced the fibrotic area as well as collagen deposition in the lung $(12,21)$.

Taken together, various studies have indicated that SFK play an important role in IPF, however, it is still unclear how exactly SFK promote profibrotic responses and which SFK member is involved. Earlier studies from our lab have indicated that TGF- $\beta 1$ is activating 
the SFK member FYN in epithelial cells but the involvement of FYN in fibroblasts is still unknown. Therefore, the aim of the present study was to explore the importance of SFK in human fibroblasts. More specifically, we investigated the role of SFK in the activation of fibroblasts as well as collagen deposition and inflammatory cytokine secretion. Additionally, the SFK members SRC, FYN and YES were targeted to determine their specific role in fibroblast activation upon stimulation with TGF- $\beta 1$. 


\section{Material and Methods}

\subsection{Cell culture and treatments}

The human fetal lung fibroblast cell line HFL-1 was purchased from the American Type Culture Collection (ATCC, Rockville, MD) and grown in Ham's F-12K Kaighn's Medium (GIBCO/Life Technologies, Bleiswijk, the Netherlands) supplemented with $10 \%$ fetal bovine serum (FBS, Gibco) at $5 \% \mathrm{CO}_{2}, 95 \%$ air and at $37^{\circ} \mathrm{C}$. Cells were starved in serumfree media overnight before stimulation with $5 \mathrm{ng} / \mathrm{mL}$ TGF- $\beta 1$ (R\&D Systems, Inc., Minneapolis, MN). Where indicated, the pharmacological inhibitor AZD0530 (2 $\mu \mathrm{M}$, Sigma Aldrich, St. Lois, MO) was administered 30 min before cell stimulation.

\subsection{Western blotting}

Cell lysates were prepared using Western solubilization buffer $(50 \mathrm{mmol} / \mathrm{L} \mathrm{HEPES}$, $250 \mathrm{mmol} / \mathrm{L} \mathrm{NaCl}, 1.5 \mathrm{mmol} / \mathrm{L} \mathrm{MgCl}_{2}, 1 \%$ Triton-X100, $10 \%$ glycerol, $1 \mathrm{mmol} / \mathrm{L}$ ethyleneglycol-bis-( $\beta$-aminoethylether)- $\mathrm{N}, \mathrm{N}, \mathrm{N}^{\prime}, \mathrm{N}^{\prime}$-tetraacetic $\quad$ acid, $\quad 1 \mathrm{mmol} / \mathrm{L}$ phenylmethylsulfonyl fluoride, $2 \mathrm{mmol} / \mathrm{L} \mathrm{Na}_{3} \mathrm{VO}_{4}, 10 \mu \mathrm{g} / \mathrm{mL}$ aprotinin, and $10 \mu \mathrm{g} / \mathrm{mL}$ leupeptin; $\mathrm{pH}$ 7.4), and protein concentration was measured by BCA protein assay kit (Thermo Scientific, Waltham, MA). Aliquots of cell lysates (containing $20 \mu \mathrm{g}$ protein) were separated on $10 \%$ precast gels (Biorad), transferred to nitrocellulose membranes and probed with antibodies against phospho-SRC family Tyr416 (1:1000) or $\beta$-ACTIN (1:5000; all from Cell Signaling, Danvers, MA). Primary antibodies were probed with rabbit-specific secondary antibodies conjugated with horseradish peroxidase (Cell Signaling) and detected by means of chemiluminescence with SuperSignal West Pico or Femto Chemiluminescent Substrate (Thermo Scientific). Integrated density of pixels in each membrane was determined using Amersham Imager 600 (GE Healthcare Life Sciences, Hillerød, Iceland) and quantified using ImageJ software $1.47 \mathrm{v}$ (W. Rasband, National Institutes of Health, Bethesda, MD).

\subsection{MTT assay}

Cell proliferation was monitored using dimethylthiazol diphenyltetrazolium bromide (MTT, Sigma-Aldrich, St. Louis, MO) colorimetric assay. Plated cells were gently washed with Hank's balanced salt solution (HBSS, GIBCO) and incubated for $1 \mathrm{~h}$ in $0.5 \mathrm{mg} / \mathrm{mL}$ MTT solution in HBSS. Thereafter, the formed formazan crystals were dissolved in dimethyl sulfoxide (DMSO) and the optical density (OD) was measured at $540 \mathrm{~nm}$. The OD percentage of treated wells to untreated control wells was considered the percentage of viable cells relative to controls.

\subsection{Small interfering RNA silencing}

At $60 \%$ confluency, HFL-1 cells were transfected with ON-TARGETplus SMARTpool small interfering RNAs (siRNAs) targeted against FYN, SRC, YES, NOX4 or non-targeting siRNA 
Pool\#1 as a control with DharmaFECT transfection reagent (Dharmacon, Lafayette, CO), according to the manufacturer's instructions, $72 \mathrm{hrs}$ before experimentation.

\subsection{RNA isolation and semi quantitative Reverse Transcription (RT)-PCR}

RNA was isolated using the RNeasy mini kit (Qiagen, Valencia, CA) according to the manufacturer's instructions. IScript cDNA Synthesis kit (Biorad, Veenendaal, the Netherlands) was used to produce cDNA from $500 \mathrm{ng}$ extracted RNA according to the manufacturer's protocol. PCR amplifications were carried out at $95^{\circ} \mathrm{C}$ for $10 \mathrm{sec}$ for denaturation and 45 cycles of annealing and elongation $\left(60^{\circ} \mathrm{C}, 20 \mathrm{sec}\right)$ for selected genes (Table 6.1). Data were retrieved using LightCycler $^{\circledR} 480$ software release 1.5.1.62 SP1 (Roche Diagnostics, Mannheim, Germany), "LC480 Conversion to LinRegPCR" software release 2014.1 and LinRegPCR version 2017.1. Quantification cycles (Cq) values were used according to the $2^{-\Delta \Delta C t}$ method to quantify the relative expression of our genes of interest.

Table 6.1. Applied human RT-PCR forward and reverse primer sequences.

\begin{tabular}{|l|l|l|}
\hline $\begin{array}{l}\text { Gene of } \\
\text { interest }\end{array}$ & Forward primer sequence & Reverse primer sequence \\
\hline COL1A1 & AAGAGGAAGGCCAAGTCGAG & CACACGTCTCGGTCATGG \\
\hline FN1-1 & CCATAAAGGGCAACCAAGAG & ACCTCGGTGTTGTAAGGTGG \\
\hline NOX4 & TGGCAAGAGAACAGACCTGA & TGGGTCCACAACAGAAAACA \\
\hline YES & AGGGGTAACGCCTTTTGGAG & ACACCACCTGTTAAACCAGCA \\
\hline FYN & TCGTCTCATACGGGGACCTT & CCAATCTCCTTCCGAGCTGT \\
\hline SRC & TTGGCAAGATCACCAGACGG & AGAGGCAGTAGGCACCTTTC \\
\hline RPL13A & CCTGGAGGAGAAGAGGAAAGAGA & TTGAGGACCTCTGTGTATTTGTCAA \\
\hline
\end{tabular}

\subsection{Enzyme-Linked Immunosorbent Assay (ELISA)}

Collagen (COL) 1A1, Interleukin (IL)-6 and IL-8 secretion in supernatants was measured using human ELISA DuoSet ${ }^{\circledR}$ kits (R\&D Systems, Inc., Minneapolis, MN) according to the protocol provided by the manufacturer.

\subsection{Statistical Analysis}

All quantitative data are shown as mean \pm SD. Means between 2 groups were analyzed by unpaired t-test whereas one-way ANOVA followed by Bonferroni comparison test was used to analyze differences among more than 2 groups using GraphPad Prism software (version 7.3; GraphPad Software, La Jolla, CA). Differences were considered significant at a $P$ value of less than 0.05 . 


\section{Results}

\subsection{SFK were activated in response to TGF- $\beta 1$ and their inhibition reduced fibroblast proliferation and activation}

TGF- $\beta 1$ induced an increase of SFK activation, shown by enhanced phosphorylation at Tyr416 in fibroblasts. To determine the effect of SFK inhibition, cells were stimulated in the presence of the SFK inhibitor AZD $(2 \mu \mathrm{M})$ for 30 min before stimulation with TGF- $\beta 1$ (5 $\mathrm{ng} / \mathrm{mL}$ ) which reduced SFK phosphorylation (Figure 6.1).

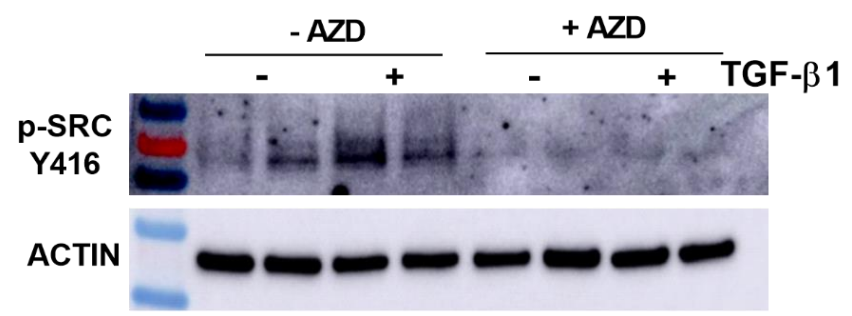

Figure 6.1: TGF-induced fibroblast proliferation and activation is dependent on SFK activation. Phosphorylation of SFK at tyrosine 416 after stimulation with TGF- $\beta 1(5 \mathrm{ng} / \mathrm{mL})$ for $1 \mathrm{hr}$ in the presence or absence of AZD $(2 \mu \mathrm{M})$ determined by Western blotting of whole cell lysates $(n=2)$.

As expected, TGF- $\beta 1$ stimulation increased the metabolic activity of fibroblasts, which is an indicator for cell proliferation (Figure 6.2A). Furthermore, TGF- $\beta 1$ induced fibroblast activation, shown by enhanced gene expression of the matrix proteins fibronectin (FN1) and collagen (COL) 1A1 (Figure 6.2B and C) as well as COL1A1 secretion (Figure 6.2D). Inhibition of SFK by AZD reduced these TGF- $\beta 1$-mediated responses indicating that SFK contribute to TGF- $\beta 1$-dependent activation of fibroblasts. 
A

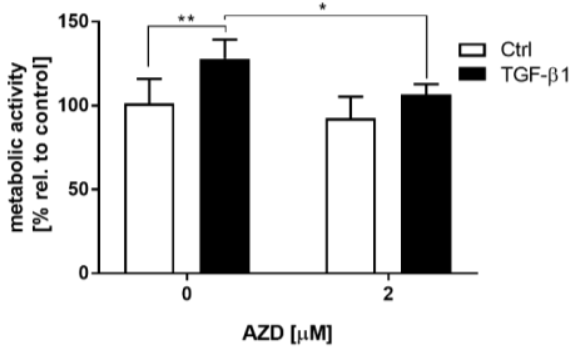

C

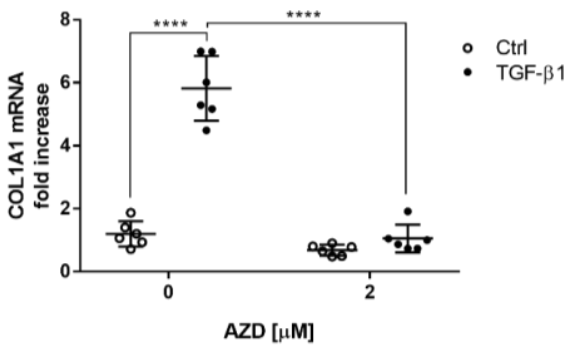

B

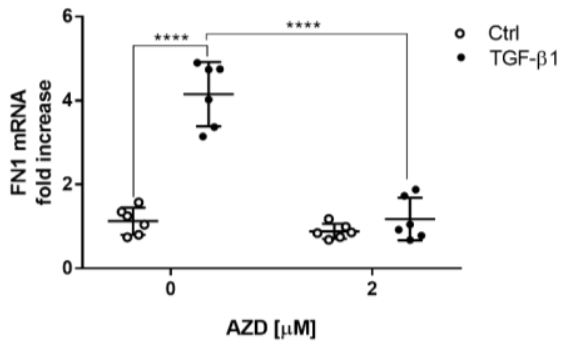

D

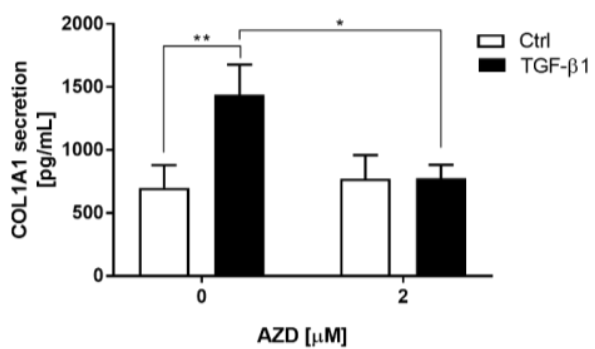

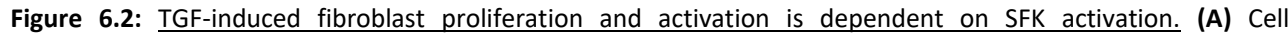
proliferation measured after stimulation with TGF- $\beta 1(5 \mathrm{ng} / \mathrm{mL})$ for $24 \mathrm{hrs}$, in the absence or presence of AZD (2 $\mu M)$ by MTT assay ( $n=6)$. Gene expression of FN1 (B) and COL1A1 (C) after stimulation with TGF- $\beta 1(5 \mathrm{ng} / \mathrm{mL})$ for $24 \mathrm{hrs}$, in the absence or presence of AZD $(2 \mu \mathrm{M})$, determined by qRT-PCR $(n=6)$. (D) Collagen secretion in supernatant after stimulation with TGF- $\beta 1(5 \mathrm{ng} / \mathrm{mL})$ for $24 \mathrm{hrs}$, in the absence or presence of AZD $(2 \mu \mathrm{M})$ measured by ELISA $(n=6)$. Results are expressed as mean \pm SD from 2-3 independent experiments $\left({ }^{*} P<0.05,{ }^{* *} P\right.$ $<0.01, * * * * P<0.0001)$.

\subsection{Pro-inflammatory cytokine release was dependent on SFK activation}

To determine if SFK are involved in TGF-induced pro-inflammatory cytokine release, we determined the secretion of interleukin (IL)- 6 and IL-8. TGF- $\beta 1$ significantly enhanced IL- 6 as well as IL- 8 release which was decreased upon inhibition of SFK by AZD (Figure 6.3A and B).

A

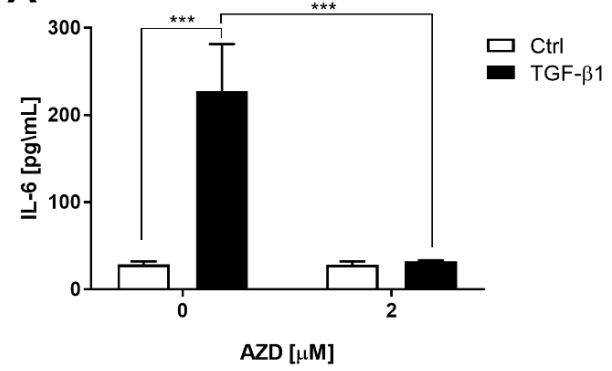

B

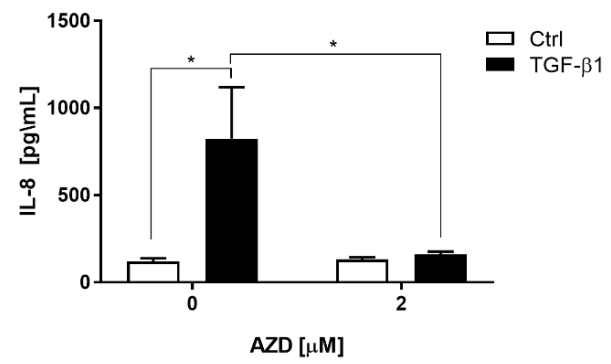

Figure 6.3: TGF-induced pro-inflammatory cytokine secretion is dependent on SFK activation. (A) IL-8 and (B) IL-6 secretion in cell supernatant $(n=6)$ after stimulation with TGF- $\beta 1(5 \mathrm{ng} / \mathrm{mL})$ for $24 \mathrm{hrs}$ in the presence or absence of AZD $(2 \mu \mathrm{M})$. ( $\left.{ }^{*} \mathrm{P}<0.05,{ }^{* * *} \mathrm{P}<0.001\right)$. 


\subsection{TGF- $\beta 1$ induced phosphorylation of the SFK member YES which promoted expression of extracellular matrix genes but not secretion of inflammatory cytokines}

From the 9 different members of the SRC family of protein tyrosine kinases SRC, FYN and YES are ubiquitously expressed in fibroblasts (22), but their expression is not increased upon TGF- $\beta 1$ stimulation (Supplemental Figure E6.1).

To determine the individual involvement of these SFK in TGF- $\beta$-mediated fibroblast activation, YES, FYN and SRC were separately silenced using small interference RNA (siRNA) (Supplemental Figure E6.2-4). Knockdown of the SFK member YES significantly reduced the TGF-mediated upregulation of $F N 1$ and COL1A1 whereas silencing FYN or SRC did not affect these responses (Figure 6.4A and B). Moreover, the TGF-induced phosphorylation of SFK was reduced upon silencing of YES (Figure 6.4 C and D). Hence, SFK-mediated profibrotic responses to TGF- $\beta 1$ may be primarily mediated by YES.

A

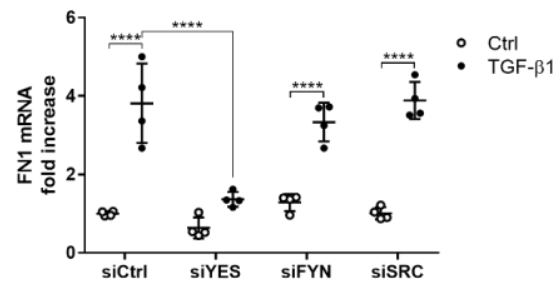

C

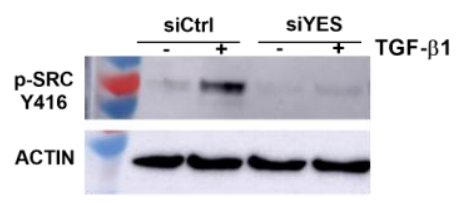

B

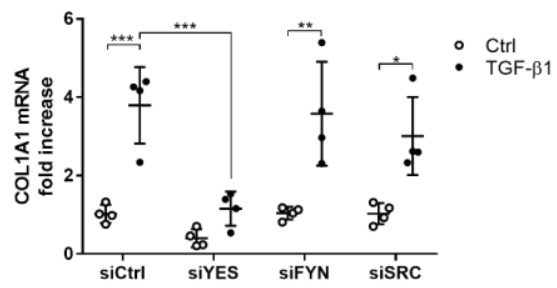

D

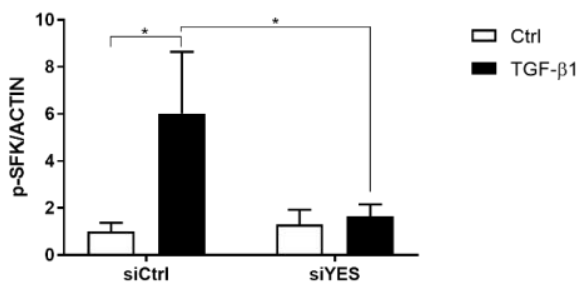

Figure 6.4: TGF- $\beta 1$ induced SFK activation and fibroblast activation is dependent on YES. (A) Gene expression of FN1 (B) and COL1A1 (D) after stimulation with TGF- $\beta 1(5 \mathrm{ng} / \mathrm{mL})$ for $24 \mathrm{hrs}$, in control and YES-silenced cells, determined by qRT-PCR $(n=6)$. (C) Knockdown of YES reduces phosphorylation at Tyr416 after stimulation with TGF- $\beta 1(5 \mathrm{ng} / \mathrm{mL})$ for $1 \mathrm{hr}$ determined by Western blotting of whole cell lysates. (D) Densitometry analysis of 2 independent experiments $(n=3)$. Results are expressed as mean \pm SD from 2-3 independent experiments $(* P<$ $\left.0.05,{ }^{* * \mathrm{P}}<0.01,{ }^{* * *} \mathrm{P}<0.001{ }^{* * * * \mathrm{P}}<0.0001\right)$.

Intriguingly, silencing of YES could not rescue the TGF-induced enhanced secretion of the pro-inflammatory cytokines IL-6 and IL-8 (Figure 6.5A and B). 
A

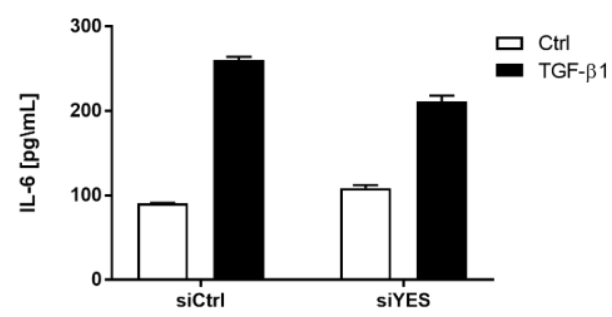

B

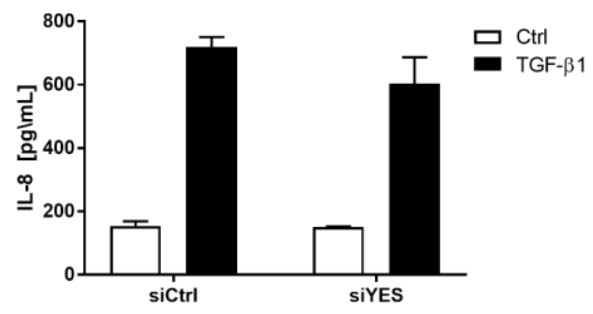

Figure 6.5: TGF-induced pro-inflammatory cytokine secretion is not dependent on YES activation. (A) IL-8 and (B) IL-6 secretion after stimulation with TGF- $\beta 1(5 \mathrm{ng} / \mathrm{mL})$ for 24 hrs in cell supernatant $(n=2)$ in control and YEStransfected cells.

\subsection{YES silencing reduced fibroblast activation by inhibiting NOX4 expression}

NOX4 is an important mediator of fibrotic responses and is known to be involved in TGFinduced fibroblast activation (9). Indeed, COL1A1 expression was reduced in NOX4 silenced cells (Supplemental Figure E6.5), suggesting that NOX4 contributes to collagen secretion (Figure 6.6A). NOX4 expression was increased upon TGF- $\beta 1$ stimulation, which was reduced in YES-transfected cells indicating that YES promotes NOX4 expression (Figure 6.6B).
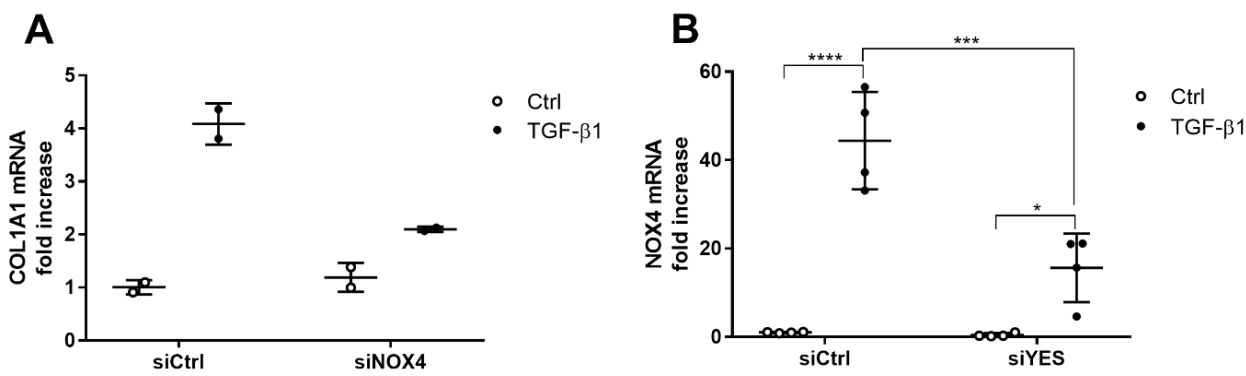

Figure 6.6: TGF- $\beta 1$ induced NOX4 activation is dependent on YES. (A) Knockdown of NOX4 reduced COL1A1 expression after stimulation with TGF- $\beta 1(5 \mathrm{ng} / \mathrm{mL})$ for $24 \mathrm{hrs}(\mathrm{n}=2)$. (B) Knockdown of YES reduces NOX4 expression after stimulation with TGF- $\beta 1(5 \mathrm{ng} / \mathrm{mL})$ for $24 \mathrm{hrs}(\mathrm{n}=4)$. Results are expressed as mean \pm SD from 2-3 independent experiments ( $\left.{ }^{*} \mathrm{P}<0.05, * * * \mathrm{P}<0.001, * * * * \mathrm{P}<0.0001\right)$. 


\section{Discussion}

We here demonstrate that SRC family kinases are mediating fibroblast activation and proinflammatory cytokine secretion. Inhibition of SFK by the pharmacological inhibitor AZD reduces TGF- $\beta 1$ induced increase in fibroblast proliferation and expression of collagen and fibronectin as well as the secretion of collagen, indicating that SFK are crucial in fibroblast proliferation and activation. Moreover, we show that TGF- $\beta 1$ induced the activation of the SFK member YES in fibroblasts as supported by the fact that phosphorylation at tyrosine 416 was abrogated in YES-silenced cells although gene expression was not changed upon TGF- $\beta 1$ stimulation. Additionally, silencing of YES reduced the increased expression of the matrix genes $F N 1$ and COL1A1 in response to TGF- $\beta 1$ but was not involved in the release of the pro-inflammatory cytokines IL- 6 and IL-8. Furthermore, YES knockdown reduced NOX4 expression which was shown to be involved in COL1A1 gene expression. Taken together, these data indicate that SFK member YES plays an important role in the activation of fibroblasts, suggesting that this kinase may provide a potential target for IPF treatment to abrogate fibroblast dependent extracellular matrix secretion.

Currently, there are two FDA-approved treatment strategies, pirfenidone and nintedanib, which can slow down the progression of the disease (23), although they cannot reverse the already induced pulmonary damage. Nintedanib mainly inhibits the platelet-derived growth factor receptor (PDGFR), fibroblast growth factor receptor (FGFR) and vascular growth factor receptor (VEGFR) (23). Interestingly, it has been indicated that nintedanib reduces ventilation-augmented bleomycin induced pulmonary fibrosis partly via the inhibition of SFK (24). Hence, it might be more beneficial to target SFK directly to prevent unwanted side-effects associated with nintedanib. Previous studies have indeed suggested a role for SFK in the development of pulmonary fibrosis as inhibition of SFK by pharmacological inhibitors reduced the fibrotic area in bleomycin induced pulmonary fibrosis in mice $(12,21)$. Furthermore, these studies have demonstrated that inhibition of SFK reduced myofibroblast activation $(12,21)$ and our results are consistent with previous findings that pharmacological inhibition of SFK reduces TGF- $\beta$ induced collagen secretion in lung fibroblasts. However, the contribution of specific SFK to fibrotic responses has not been addressed thus far. Our findings indicate that TGF- $\beta 1$ induced activation of fibroblasts is mediated by the SFK member YES. However, this finding is not excluding that other SFK are also involved as it has been suggested previously that different members have overlapping functions and that other SFK are also involved in in vivo fibrotic remodeling. For instance, FYN has been shown to contribute to epithelial-to-mesenchymal transition in mouse hepatic cells (25). It has also been shown that FYN is activated in a mice renal fibrosis model (26) and silencing of this kinase ameliorated renal fibrosis (26). Furthermore, recent studies from our lab have indicated that FYN is activated in response 
to TGF- $\beta 1$ and mediated the induction of DNA damage as well as mitochondrial ROS production in human bronchial epithelial cells (chapter 5 , unpublished data).

However, it is still not completely clear how SFK specifically promote fibroblast activation. It has been suggested that SFK regulate myofibroblast differentiation through phosphorylation of focal adhesion kinase (FAK) at tyrosine 397 (12). Other studies have indicated that SFK induce SMAD signaling which in turn promotes fibrotic gene expression which was assessed in SRC, YES, FYN triple silenced mouse fibroblasts (27). Furthermore, it has been demonstrated that the SRC inhibitor KX2-391 reduces collagen and myofibroblast activation as well as pro-inflammatory cytokine secretion in a rat model of chlorhexidine gluconate induced peritoneal fibrosis (28). This study also indicated that SRC is inducing TGF-mediated SMAD3 activation (28). All these various lines of evidence support a role for SFK in fibrotic signaling but different members of the SFK may mediate different aspects of TGF- $\beta$ induced responses.

Our results show that SFK are involved in pro-inflammatory cytokine secretion, however, the increase in pro-inflammatory cytokines release in response to TGF- $\beta 1$ is not mediated by YES. These observations suggest that the secretion of IL- 6 and IL- 8 is mediated by a different member of the family of SRC kinases. Previous studies have indicated that the activation of the SFK member Hck is associated with inflammatory cell infiltration and mild emphysema after systemic administration of lipopolysaccharide in a model of endotoxemia in mice (29). Furthermore, SRC has been identified to contribute to inflammatory cytokine secretion in acute lung injury via the induction of SMAD3 (30). However, more studies need to be performed to identify SFK members(s) involved in proinflammatory cytokine secretion in pulmonary fibrosis.

NOX4-dependent $\mathrm{H}_{2} \mathrm{O}_{2}$ production has been implicated in the activation of fibroblasts (31) and NOX4 knockout mice are protected from bleomycin-induced pulmonary fibrosis (9). Furthermore, since SFK can be activated through redox-dependent mechanisms (32), SFKdependent regulation of NOX4 could be an important target for IPF treatment. It has been suggested before that SFK can regulate the activity of NOX4 (33). A previous study has shown that the SFK member FYN can negatively regulate the activity of NOX4 through phosphorylation at tyrosine 566 in cardiomyocytes (33). Our data indicate that SFK, in specific, YES regulates the expression of NOX4. This finding suggests that a different mechanism of action might be involved as SFK activation is also increased in HBE cells from IPF patients (C Veith, unpublished data) but NOX4 is highly upregulated in fibroblasts from IPF patients (31). Interestingly, it has been shown that SFK can phosphorylate the NOX co-activator $\mathrm{p} 47^{\text {phox }}$ resulting in enhanced NOX activity in human endothelial cells and inhibition of SFK by PP2 attenuated activation of SFK as well as ROS production (34). This finding suggests that other cofactors, for instance $\mathrm{p} 22^{\text {phox }}$ which mediates the activation of NOX4 (35), can be phosphorylated as well. Indeed, p22 ${ }^{\text {phox }}$ can be phosphorylated at tyrosine residues which also correlates with NOX activation in 
neutrophils (36), however, the role of SFK in $\mathrm{p} 22^{\text {phox }}$ phosphorylation is unclear. Furthermore, SFK can also phosphorylate promotor regions thereby regulating the transcription of genes. Consequently, it would be of interest to further explore the role of YES in the activation of NOX4 (37) to reduce NOX4-dependent ROS production.

Taken together, our study supports a role for SFK in fibroblast proliferation and activation. Furthermore, we have identified that the SFK member is phosphorylated in response to the pro-fibrotic trigger TGF- $\beta 1$ and induces the expression of FN1 and COL1A1 in human fibroblasts. Interestingly, YES does not promote pro-inflammatory cytokine secretion, suggesting that it might be more beneficial to inhibit various SFK members instead of specifically targeting one member to counteract fibroblast-mediated remodeling in the lungs of IPF patients. 


\section{References}

1. Raghu G, Collard HR, Egan JJ, Martinez FJ, Behr J, Brown KK, et al. An official ATS/ERS/JRS/ALAT statement: idiopathic pulmonary fibrosis: evidence-based guidelines for diagnosis and management. Am J Respir Crit Care Med. 2011;183(6):788-824.

2. du Bois RM. Strategies for treating idiopathic pulmonary fibrosis. Nature reviews Drug discovery. 2010;9(2):129-40.

3. Hecker L, Cheng J, Thannickal VJ. Targeting NOX enzymes in pulmonary fibrosis. Cell Mol Life Sci. 2012;69(14):2365-71.

4. Hinz B. Formation and function of the myofibroblast during tissue repair. J Invest Dermatol. 2007;127(3):526-37.

5. Kendall RT, Feghali-Bostwick CA. Fibroblasts in fibrosis: novel roles and mediators. Front Pharmacol. 2014;5:123.

6. Buckley CD, Pilling D, Lord JM, Akbar AN, Scheel-Toellner D, Salmon M. Fibroblasts regulate the switch from acute resolving to chronic persistent inflammation. Trends Immunol. 2001;22(4):199-204.

7. Kurundkar A, Thannickal VJ. Redox mechanisms in age-related lung fibrosis. Redox Biol. 2016;9:67-76.

8. Cheresh P, Kim SJ, Tulasiram S, Kamp DW. Oxidative stress and pulmonary fibrosis. Biochim Biophys Acta. 2013;1832(7):1028-40.

9. Hecker L, Vittal R, Jones T, Jagirdar R, Luckhardt TR, Horowitz JC, et al. NADPH oxidase-4 mediates myofibroblast activation and fibrogenic responses to lung injury. Nat Med.

2009;15(9):1077-81.

10. Fernandez IE, Eickelberg $\mathrm{O}$. The impact of TGF-beta on lung fibrosis: from targeting to biomarkers. Proc Am Thorac Soc. 2012;9(3):111-6.

11. Jiang F, Liu GS, Dusting GJ, Chan EC. NADPH oxidase-dependent redox signaling in TGFbeta-mediated fibrotic responses. Redox Biol. 2014;2:267-72.

12. Hu M, Che P, Han X, Cai GQ, Liu G, Antony V, et al. Therapeutic targeting of SRC kinase in myofibroblast differentiation and pulmonary fibrosis. The Journal of pharmacology and experimental therapeutics. 2014;351(1):87-95.

13. Abdalla M, Thompson L, Gurley E, Burke S, Ujjin J, Newsome R, et al. Dasatinib inhibits TGFbeta-induced myofibroblast differentiation through Src-SRF Pathway. Eur J Pharmacol. 2015;769:134-42.

14. Zhang H, Davies KJ, Forman HJ. TGFbeta1 rapidly activates Src through a non-canonical redox signaling mechanism. Arch Biochem Biophys. 2015;568:1-7.

15. Meng Y, Pond MP, Roux B. Tyrosine Kinase Activation and Conformational Flexibility: Lessons from Src-Family Tyrosine Kinases. Acc Chem Res. 2017;50(5):1193-201.

16. Roskoski R, Jr. Src protein-tyrosine kinase structure, mechanism, and small molecule inhibitors. Pharmacol Res. 2015;94:9-25.

17. Thomas SM, Brugge JS. Cellular functions regulated by Src family kinases. Annu Rev Cell Dev Biol. 1997;13:513-609.

18. Harrison SC. Variation on an Src-like theme. Cell. 2003;112(6):737-40.

19. Roskoski R, Jr. Src protein-tyrosine kinase structure and regulation. Biochem Biophys Res Commun. 2004;324(4):1155-64.

20. Giannoni E, Taddei ML, Chiarugi P. Src redox regulation: again in the front line. Free Radic Biol Med. 2010;49(4):516-27.

21. Lu YY, Zhao XK, Yu L, Qi F, Zhai B, Gao CQ, et al. Interaction of Src and Alpha-V Integrin Regulates Fibroblast Migration and Modulates Lung Fibrosis in A Preclinical Model of Lung Fibrosis. Sci Rep. 2017;7:46357. 
22. Courtneidge SA, Fumagalli S, KoegI M, Superti-Furga G, Twamley-Stein GM. The Src family of protein tyrosine kinases: regulation and functions. Dev Suppl. 1993:57-64.

23. Kolb M, Bonella F, Wollin L. Therapeutic targets in idiopathic pulmonary fibrosis. Respiratory medicine. 2017;131:49-57.

24. Li LF, Kao KC, Liu YY, Lin CW, Chen NH, Lee CS, et al. Nintedanib reduces ventilationaugmented bleomycin-induced epithelial-mesenchymal transition and lung fibrosis through suppression of the Src pathway. J Cell Mol Med. 2017;21(11):2937-49.

25. Zhao XK, Yu L, Cheng ML, Che P, Lu YY, Zhang Q, et al. Focal Adhesion Kinase Regulates Hepatic Stellate Cell Activation and Liver Fibrosis. Sci Rep. 2017;7(1):4032.

26. Seo HY, Jeon JH, Jung YA, Jung GS, Lee EJ, Choi YK, et al. Fyn deficiency attenuates renal fibrosis by inhibition of phospho-STAT3. Kidney Int. 2016;90(6):1285-97.

27. Samarakoon R, Chitnis SS, Higgins SP, Higgins CE, Krepinsky JC, Higgins PJ. Redox-induced Src kinase and caveolin-1 signaling in TGF-beta1-initiated SMAD2/3 activation and PAI-1 expression. PLoS One. 2011;6(7):e22896.

28. Wang J, Wang L, Xu L, Shi Y, Liu F, Qi H, et al. Targeting Src attenuates peritoneal fibrosis and inhibits the epithelial to mesenchymal transition. Oncotarget. 2017;8(48):83872-89.

29. Ernst M, Inglese M, Scholz GM, Harder KW, Clay FJ, Bozinovski S, et al. Constitutive activation of the SRC family kinase Hck results in spontaneous pulmonary inflammation and an enhanced innate immune response. J Exp Med. 2002;196(5):589-604.

30. Li LF, Lee CS, Liu YY, Chang CH, Lin CW, Chiu LC, et al. Activation of Src-dependent Smad3 signaling mediates the neutrophilic inflammation and oxidative stress in hyperoxia-augmented ventilator-induced lung injury. Respir Res. 2015;16:112.

31. Amara N, Goven D, Prost F, Muloway R, Crestani B, Boczkowski J. NOX4/NADPH oxidase expression is increased in pulmonary fibroblasts from patients with idiopathic pulmonary fibrosis and mediates TGFbeta1-induced fibroblast differentiation into myofibroblasts. Thorax. 2010;65(8):733-8.

32. Heppner DE, Dustin CM, Liao C, Hristova M, Veith C, Little AC, et al. Direct cysteine sulfenylation drives activation of the Src kinase. Nat Commun. 2018;9(1):4522.

33. Matsushima S, Kuroda J, Zhai P, Liu T, Ikeda S, Nagarajan N, et al. Tyrosine kinase FYN negatively regulates NOX4 in cardiac remodeling. J Clin Invest. 2016;126(9):3403-16.

34. Chowdhury AK, Watkins T, Parinandi NL, Saatian B, Kleinberg ME, Usatyuk PV, et al. Srcmediated tyrosine phosphorylation of p47phox in hyperoxia-induced activation of NADPH oxidase and generation of reactive oxygen species in lung endothelial cells. J Biol Chem.

2005;280(21):20700-11.

35. Martyn KD, Frederick LM, von Loehneysen K, Dinauer MC, Knaus UG. Functional analysis of Nox4 reveals unique characteristics compared to other NADPH oxidases. Cell Signal. 2006;18(1):69-

82.

36. Regier DS, Greene DG, Sergeant S, Jesaitis AJ, McPhail LC. Phosphorylation of p22phox is mediated by phospholipase D-dependent and -independent mechanisms. Correlation of NADPH oxidase activity and p22phox phosphorylation. J Biol Chem. 2000;275(37):28406-12.

37. Fujii M, Shalloway D, Verma IM. Gene regulation by tyrosine kinases: src protein activates various promoters, including c-fos. Mol Cell Biol. 1989;9(6):2493-9. 


\section{Supplemental data}

Figure E6.1: YES, FYN and SRC expression in HFL-1 cells. Gene expression of YES, FYN and $S R C$ relative to RPL13 in HFL-1 cells stimulated with TGF- $\beta 1$ for $24 \mathrm{~h}$, determined using qRT$\operatorname{PCR}(n=4)$.

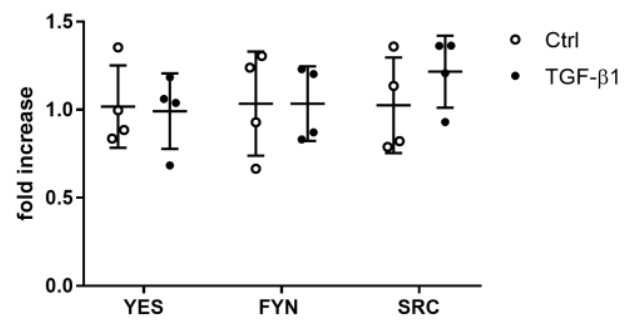

Figure E6.2: Evaluation of siRNA-mediated silencing of YES mRNA in HFL-1 cells. Gene expression of YES relative to RPL13 in HFL-1 cells transfected with siCtrl or siYES, determined using qRT-PCR $(n=4)$. Results are expressed as mean \pm SD (****P<0.0001).

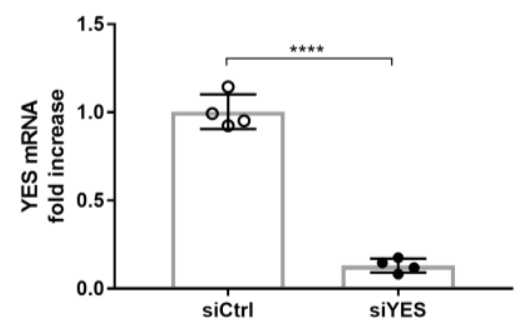

Figure E6.3: Evaluation of siRNA-mediated silencing of FYN mRNA in HFL-1 cells. Gene expression of FYN relative to RPL13 in HFL-1 cells transfected with siCtrl or siFYN, determined using qRT-PCR $(n=4)$. Results are expressed as mean $\pm S D(* * * * P<0.0001)$. 


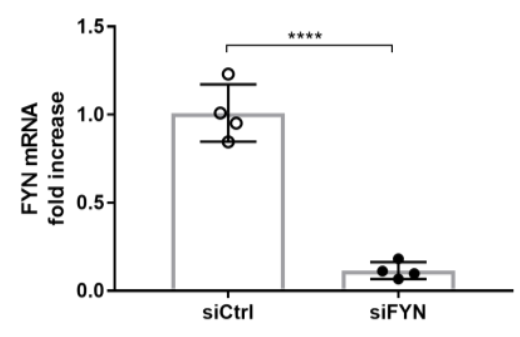

Figure E6.4: Evaluation of siRNA-mediated silencing of SRC mRNA in HFL-1 cells. Gene expression of $S R C$ relative to RPL13 in HFL-1 cells transfected with siCtrl or siSRC, determined using qRT-PCR $(n=4)$. Results are expressed as mean \pm SD (***P<0.001).

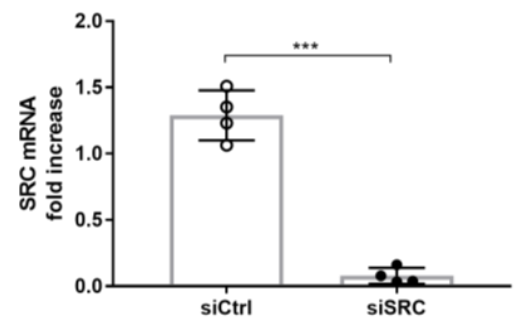

Figure E6.5: Evaluation of siRNA-mediated silencing of NOX4 mRNA in HFL-1 cells. Gene expression of NOX4 relative to RPL13 in HFL-1 cells transfected with siCtrl or siNOX4, determined using qRT-PCR ( $n=3-4)$. Results are expressed as mean $\pm S D(* * * P<0.001)$.

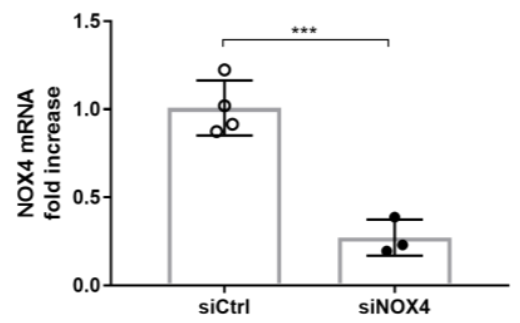





\section{Chapter 7}

Inhibition of SRC family kinases alleviates impaired expression levels of key regulators of mitochondrial biogenesis and mitophagy in bronchial epithelial cells of IPF patients

Carmen Veith, Nicolas Kahn, Alex Remels, Lou Maas, Felix J.F. Herth, Michael Kreuter, Marc Schneider, Michael Meister, Albert van der Vliet, Frederik-Jan van Schooten and Agnes W. Boots.

In preparation 


\section{Abstract}

Rationale: Mitochondria are critical for cellular homeostasis and when dysfunctional they can contribute to cellular stress and apoptosis. Idiopathic pulmonary fibrosis (IPF) is characterized by increased production of reactive oxygen species, also by the NADPH oxidase NOX4, that among others promote mitochondrial dysfunction. Recently, SRC family kinases (SFK) have been suggested as treatment strategy for IPF. However, it is still unclear whether SFK activation is increased in IPF patients and if so, whether they are involved in IPF pathology. In this study we investigated whether SFK are activated in human bronchial epithelial (HBE) cells derived from IPF patients and if modulation of SFK interferes with molecular pathways controlling mitochondrial function. Additionally, the role of NOX4 herein will be explored.

Methods: In our study, HBE cells were obtained from epithelial lining fluid from controls $(n=3)$ and IPF patients $(n=4)$ by bronchoscopic microsampling. Afterwards, HBE cells were expanded and SFK activation, key regulators of mitochondrial biogenesis and mitophagy as well as NOX4 expression and cell damage were evaluated in the presence or absence of the SFK inhibitor AZD.

Results: Here, we demonstrate that HBE cells derived from IPF patients have an increased activation of SFK compared to HBE cells from non-IPF patients which was reduced upon treatment with AZD in both the IPF and non-IPF derived HBE cells. Inhibition of SFK in HBE cells from IPF patients increased the mitochondrial biogenesis markers NRF2, PGC-1 $\beta$ and ERR $\alpha$ and the mitophagy marker PINK1 which were decreased in HBE cells from IPF patients. Inhibition of SFK also rescued the increased NOX4 expression in HBE cells from IPF patients. Moreover, AZD reduced the increased lactate dehydrogenase (LDH) release and expression of the pro-apoptotic BAX in HBE cells derived from IPF patients.

Conclusion: Our results suggest that SFK activation is increased in bronchial epithelial cells from IPF patients and that this activation is associated with reduced expression levels of key regulators of mitochondrial biogenesis. Inhibition of SFK can restore expression of these mitochondrial biogenesis and mitophagy regulators, suggesting that SFK might be a therapeutic target to improve mitochondria health in IPF.

Keywords: IPF, primary bronchial epithelial cells, NOX4, SRC family kinases, mitochondrial dysfunction 


\section{Introduction}

Idiopathic pulmonary fibrosis (IPF) is a progressive and irreversible lung disease of unknown etiology with a median survival of 2-4 years after diagnosis due to respiratory failure (1). IPF is driven by recurrent injury to the lung epithelium, leading to aberrant wound healing responses that eventually cause over-activation of myofibroblasts and lead to epithelial cell apoptosis (2). Indeed, injury to the lung epithelium is now seen as an early event in IPF development (3).

Recently, SRC family kinases (SFK) have been implicated in the development of IPF and inhibition of SFK by general pharmacological inhibitors improves markers of bleomycininduced pulmonary fibrosis in vivo $(4,5)$. There are 11 known members of the SRC family, i.e. CSRC, FYN, YES, BLK, BRK, FGR, FRK, HCK, LCK, LYN, SRM (6) that regulate various physiological functions including cell proliferation and survival (7). SFK are mainly activated via their phosphorylation status (8), but oxidation of their cysteine residues can also be involved $(9,10)$. Although SFK are mainly located in the cytoplasm, it has been shown that several SFK including C-SRC, FYN, LYN and LCK are also either present in or translocate into the mitochondria through specific adaptor proteins (11-13). Mitochondrial proteins can be phosphorylated by SFK, which will regulate their activity, as it has already shown that over 100 mitochondrial proteins indeed undergo tyrosinephosphorylation (14). More specifically, the SFK members such as CSRC and FYN can phosphorylate subunits of mitochondrial complex I, II and IV of the electron transport chain (ETC) as well as pyruvate dehydrogenase thereby regulating oxidative phosphorylation (OXPHOS) $(11,12)$ and consequently mitochondrial function.

Under normal conditions, mitochondrial mass and function is tightly regulated through a balance between mitochondrial biogenesis and mitophagy, respectively the production and breakdown of mitochondria. Mitochondrial biogenesis is regulated by peroxisome proliferator-activated receptor (PPAR) $\gamma$, coactivator-1 $\alpha$ (PGC-1 $\alpha$ ) and PGC-1 $\beta$ which activate nuclear respiratory factor (NRF) 1 and NRF2. NRF1 and NRF2 regulate the expression of various nuclear and mitochondrial genes such as the mitochondrial transcription factor A (TFAM) which is responsible for mitochondrial DNA replication during mitochondrial biogenesis. PGC-1 also interacts with other transcription factors including PPAR $\alpha$ and PPAR $\delta$ as well as estrogen-related receptor (ERR) $\alpha$ (15). ERR $\alpha$ is a nuclear receptor that is involved in mitochondrial biogenesis as well as regulation of fatty acid oxidation and oxidative phosphorylation (16). Normally, dysfunctional mitochondria are broken down by a process called mitophagy, which is regulated through PTEN/induced putative kinase 1 (PINK1) and the E3 ubiquitin ligase PARK2/Parkin, which prime the mitochondria for breakdown (17). Interestingly, it has been shown that PINK1 expression is decreased in AECs of IPF patients, thereby impairing mitochondrial homeostasis (18). Additionally, PINK1 deficient mice are more prone to develop 
pulmonary fibrosis while displaying increased apoptosis of AECs (19). Mitophagy can also be regulated through mitophagy-receptors on the outer membrane of the mitochondria like BCL2/Adenovirus E1B $19 \mathrm{kDa}$ protein-interacting protein 3 (BNIP3) and BNIP3-like (BNIP3L) (20). Furthermore, mitophagy also regulates mtROS production indirectly by removing dysfunctional mitochondria with high mtROS production (21).

Dysbalance of these regulatory mechanisms disrupts adaption to cellular stress, making epithelial cells more vulnerable to injury and thus promoting cell death which is regulated through the extrinsic pathway, mainly involving cell surface receptors, and the intrinsic, mitochondria-regulated pathway. Mitochondria-regulated apoptosis involves the balance between pro-apoptotic and anti-apoptotic proteins, the B-cell lymphoma ( $B C L)-2$ proteins (22). BCL-2 promotes cell survival whereas BCL-2 associated $\mathrm{X}$ protein (BAX) promotes cell death through the permeabilization of the outer membrane of mitochondria causing the release of cytochrome $c$ into the cytoplasm thereby activating caspases (23).

Additionally, the ROS-producing NADPH oxidase (NOX) 4, which is upregulated in IPF patients, has been shown to be present in mitochondria (24) and represses mitochondrial biogenesis through inhibition of NRF2 and TFAM (25). Impaired biogenesis results in an imbalance between cellular energy demand and production capacity, leading to the accumulation of dysfunctional mitochondria. Mitochondrial dysfunction, characterized by an increased production of mitochondrial reactive oxygen species (ROS) and altered mitochondrial dynamics, may represent an important mechanism of AEC apoptosis (26). Indeed, IPF patients have more dysfunctional and dysmorphic mitochondria in type II AEC compared to non-IPF patients (18).

The potential activation of SFKs in IPF epithelial cells as well as its alleged involvement in mitochondrial homeostasis, and the possible role of NOX4 herein, are not yet fully elucidated. Therefore, the aim of this study was to first investigate whether SFK are activated in human bronchial epithelial (HBE) cells derived from IPF patients. With respect to mitochondrial funtion, the expression of markers of mitochondrial biogenesis (NRF1, NRF2, PGC1 $\beta$, ERR $\alpha$, PPAR $\alpha$, PPAR $\delta$ and TFAM), mitophagy (PINK1, PARK2, BNIP3, $B N I P 3 L$ ) as well as mitochondrial apoptosis protein (BAX and $B C L 2$ ) were compared between HBE cells from IPF patients and controls. Furthermore, we explored the role of NOX4. 


\section{Material and Methods}

\subsection{Bronchoscopic microsampling}

Human bronchial epithelial (HBE) cells were obtained from endobronchial lining fluid (ELF) of IPF patients and controls by minimally invasive bronchoscopic microsampling (BMS) during bronchoscopy from subsegmental airways as previously described (27). All participants gave written informed consent, and the study was approved by the ethics committee of the University of Heidelberg, Germany.

\subsection{Patient's characteristics}

In total 7 patients were enrolled in this study via recruitment at the Center for interstitial and rare lung diseases, Thoraxklinik, University of Heidelberg (see Table 7.1 for their characteristics). All patients gave their written informed consent.

\section{Lung fibrosis patients}

IPF was diagnosed according to the ATS-ERS consensus criteria (28) in 4 male patients (see Table 7.1 for their characteristics). Two were ex-smokers, 1 never smoked and 1 was an active smoker. At the time of sampling, none of the patient received treatment yet.

\section{Control patients (non-IPF)}

For the control group, 3 patients ( 2 males, 1 female) undergoing bronchoscopy for further investigation of indeterminate pulmonary nodules were included of whom the main characteristics are shown in Table 8.1. ELF was obtained from a noninvolved segment, from the contralateral lung, opposite the solitary lesion to minimize possible influences of the suspected malignancy. Two patients were smokers and 1 was an ex-smoker.

Table 7.1: Patient characteristics. Age is expressed in years, DLCO (diffuse capacity of the lung for carbon monoxide), $\mathrm{FEV}_{1}$ (forced expiratory volume in $1 \mathrm{~s}$ ) and FVC (forced vital capacity) in percentage of the predicted value based on age and gender. Data are expressed as range (mean $\pm S D)$. (* $P<0.05, * * * P<0.001)$

\begin{tabular}{|c|c|c|}
\hline & $\begin{array}{c}\text { IPF patients } \\
(\mathbf{n}=\mathbf{4})\end{array}$ & $\begin{array}{c}\text { Controls, } \text { non-IPF } \\
\text { patients (n = 3) }\end{array}$ \\
\hline Gender (m/f) & $4(4 / 0)$ & $3(2 / 1)$ \\
\hline Age (years) & $67-73(72 \pm 3.9)^{* * *}$ & $53-55(54 \pm 1.2)$ \\
\hline DLCO (\% predicted) & $36.5-48.4(41 \pm 5.3)^{* * *}$ & $81.9-101.8(94 \pm 11)$ \\
\hline FVC (\% predicted) & $60.9-89.7(80 \pm 14)^{*}$ & $93-113.3(106 \pm 12)$ \\
\hline $\begin{array}{c}\text { Smoking status } \\
\text { (never, ex, current) }\end{array}$ & $1,2,1$ & $0,2,1$ \\
\hline
\end{tabular}




\subsection{Cell culturing and cell treatment}

Primary HBE cells were centrifuged for $5 \mathrm{~min}$ at room temperature and $300 \times \mathrm{g}$ to elute sampled cells from sponges. Afterwards, cells were resuspended and expanded in DMEM/F12 media (Gibco, Carlsbad, CA) supplemented with bovine pituitary extract $(0.004 \mathrm{~mL} / \mathrm{mL})$, epidermal growth factor $(10 \mathrm{ng} / \mathrm{mL})$, insulin $(5 \mu \mathrm{g} / \mathrm{mL})$, hydrocortisone $(0.5$ $\mu \mathrm{g} / \mathrm{mL})$, triiodo-L-thyronine $(6.7 \mathrm{ng} / \mathrm{mL})$ and transferrin $(10 \mu \mathrm{g} / \mathrm{mL})$ (PromoCell, Heidelberg, Germany), sodium selenite (30 nM, Sigma), ethanolamine (10 $\mu \mathrm{M}$, Sigma), phosphorylethanolamine (10 $\mu \mathrm{M}$, Sigma), sodium pyruvate $(0.5 \mu \mathrm{M}, \mathrm{Gibco})$, adenine $(0.18$ $\mathrm{mM}$, Sigma), hepes (15 mM, Gibco), 1x GlutaMAX (Gibco) and in the presence of $10 \mu \mathrm{M}$ Rock-inhibitor (StemCell, Cologne, Germany), as described previously (29). Cells were seeded in 24-well plates and after reaching $90 \%$ confluency, starved in EGF-free media for $24 \mathrm{hrs}$ prior to experiments. Cells were stimulated with the pharmacological SFK inhibitor AZD0530 (1 $\mu \mathrm{M}$, Selleck Chemicals, Houston, TX) and harvested at appropriate time points for analysis of SFK expression and activation, oxidative and mitochondrial biogenesis gene expression.

\subsection{LDH assay}

Lactate dehydrogenase (LDH) cell death assay was used according to the instructions of the manufacturer (Thermo Scientific, Waltham, MA).

\subsection{RNA isolation and RT-PCR}

RNA was isolated and purified using the RNeasy mini kit (Qiagen, Venlo, the Netherlands) according to the manufacturer's instructions. The RNA concentration was determined using a Nanodrop spectrophotometer (Thermo Scientific) after which cDNA was synthesized from $1 \mathrm{mg}$ isolated RNA using IScript (Biorad, Hercules, CA) according to the manufacturer's instructions. RT-PCR was performed using Sensimix SYBR (Bioline, Waddinxveen, the Netherlands) with $4.4 \mu \mathrm{L}$ of 50 times diluted cDNA and $0.5 \mu \mathrm{mol} / \mathrm{L}$ predesigned primers (Table 7.2). PCR amplifications were carried out for up to 55 cycles of denaturation $\left(95^{\circ} \mathrm{C}, 10 \mathrm{sec}\right)$ and annealing/elongation $\left(60^{\circ} \mathrm{C}, 60 \mathrm{sec}\right)$ using a LightCycler480 system (Roche, Almere, the Netherlands). The gene expression was normalized to the house keeping genes GAPDH, B2M, RPL13A and CycloA using GeNorm correction factor. 
Table 7.2.: Human RT-PCR forward and reverse primer sequences.

\begin{tabular}{|c|c|c|}
\hline $\begin{array}{l}\text { Gene of } \\
\text { interest }\end{array}$ & Forward primer sequence & Reverse primer sequence \\
\hline GAPDH & GCACCACCAACTGCTTAGCA & TGGCAGTGATGGCATGGA \\
\hline B2M & CTGTGCTCGCGCTACTCTCTCTT & $\begin{array}{l}\text { TGAGTAAACCTGAATCTTTGGAGTAC } \\
\text { GC }\end{array}$ \\
\hline RLP13A & CCTGGAGGAGAAGAGGAAAGAGA & TTGAGGACCTCTGTGTATTTGTCAA \\
\hline CycloA & CATCTGCACTGCCAAGACTGA & TTCATGCCTTCTTTCACTTTGC \\
\hline PGC1- $\alpha$ & AAGCCACTACAGACACCGC & TCGTAGCTGTCATACCTGGG \\
\hline PGC1- $\beta$ & GGCGCTTTGAAGTGTTTGGTGA & TGATGAAGCCGTACTTCTCGCCT \\
\hline NRF1 & GCACCTTTGGAGAATGTGGT & CTGGGATAAATGCCCGAAG \\
\hline $\begin{array}{l}\text { NRF2 } \alpha / G A B P \\
A\end{array}$ & CTCACCTGGGAACAGAACAGGAA & ACCCAAGAAATGCAGTCTCGAGC \\
\hline TFAM & $\begin{array}{l}\text { GAAAGATTCCAAGAAGCTAAGGGTG } \\
\text { ATT }\end{array}$ & $\begin{array}{l}\text { TCCAGTTTTCCTTTACAGTCTTCAGCT } \\
\text { TTT }\end{array}$ \\
\hline $\mathrm{ERR} \alpha$ & TGCTGCTCACGCTACCGCTC & TCGAGCATCTCCAAGAACAGC \\
\hline PPAR $\alpha$ & CAGAACAAGGAGGCGGAGGTC & AGGTCCAAGTTTGCGAAGC \\
\hline PPARס & TGACCAAAAAGAAGGCCCGC & GTCGTGGATCACAAAGGGCG \\
\hline PINK1 & GAAAGCCGCAGCTACCAAGA & AGCACATTTGCGGCTACTCG \\
\hline PARK2 & GGTTTGCCTTCTGCCGGGAATG & CTTTCATCGACTCTGTAGGCCTG \\
\hline BNIP3 & AGCGCCCGGGATGCA & CCCGTTCCCATTATTGCTGAA \\
\hline BNIP3L & CTGCGAGGAAAATGAGCAGTCTCT & GCCCCCCATTTTTCCCATTG \\
\hline FUNDC1 & GAAACGAGCGAACAAAGCAG & GCAAAAAGCCTCCCACAAAT \\
\hline NOX4 & GGTAAGCCAAGAGTGTTCGG & ACCAAGGGCCAGAGTATCAC \\
\hline BAX & GGTCTTTTTCCGAGTGGCAG & CACAGGGCCTTGAGCAC \\
\hline $\mathrm{BCL} 2$ & GTCTTTTTCCGAGTGGCAGC & GTAGAAAAGGGCGACAACCC \\
\hline
\end{tabular}




\subsection{Protein extraction and Western blotting}

Cell lysates were prepared using RIPA buffer (Thermo Scientific) containing $1 x$ phosphatase and protease inhibitors (Thermo Scientific) and $1 \mathrm{mmol} / \mathrm{L}$ phenylmethylsulfonyl fluoride (PMSF). Protein concentration was measured with BCA protein assay kit (Thermo Scientific). Aliquots of cell lysates containing $10 \mu \mathrm{g}$ protein were separated on 10\% SDS-PAGE gels (Biorad), transferred to nitrocellulose membranes and probed with antibodies against pSFK (1:1000) or $\beta$-ACTIN (1:5000; all from Cell Signaling, Danvers, MA). Primary antibodies were probed with anti-rabbit HRP-linked secondary antibody (Cell Signaling, Danvers, MA) and detected by means of chemiluminescence with SuperSignal ${ }^{\mathrm{TM}}$ West Pico PLUS or SuperSignal ${ }^{\mathrm{TM}}$ West Femto Maximum Sensitivity Substrate (Thermo Scientific) using Amersham Imager 600 (GE Healthcare Life Sciences, Hillerød, Iceland). Integrated density of pixels in each membrane was quantified using ImageJ software 1.47v (W. Rasband, National Institutes of Health, Bethesda, MD).

\subsection{Statistical Analysis}

All quantitative data are represented as means \pm SD. Statistical differences between groups were evaluated by means of Student's t-test for normal distributed variables and Mann-Whitney test for non-normal distributed variables in GraphPad Prism software (version 7.3; GraphPad Software, La Jolla, CA). Differences were considered significant at a $P$ value equal to or less than 0.05 . 


\section{Results}

\subsection{SFK are activated in bronchial epithelial cells from IPF patients}

HBE cells from IPF patients display an increased activation of SFK, shown by increased phosphorylation at Tyr416 (Figure 7.1), which can be inhibited by treating HBE cells with AZD for 24 hrs (Figure 7.1).

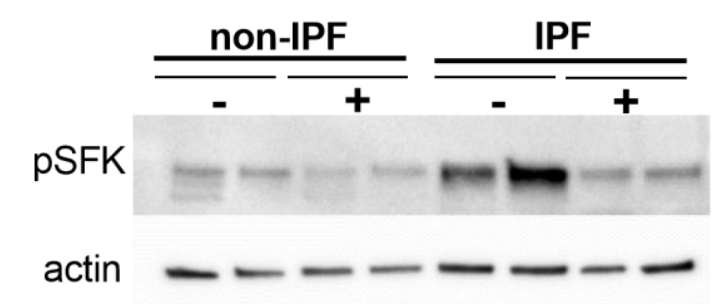

Figure 7.1: Increased activation of SFK in HBE cells from IPF patients. Phosphorylation of tyrosine 416 in the absence $(-)$ and presence $(+)$ of AZD $(1 \mu \mathrm{M})$ for 24 hrs in HBE cells derived from non-IPF and IPF patients.

\subsection{HBE cells from IPF patients express low levels of regulators of mitochondrial function}

It has been suggested that SFK can locate to mitochondria (12) where they regulate mitochondrial function through protein phosphorylation $(30,31)$. As it is already known that AECII of IPF patients display features indicative of mitochondrial dysfunction (18), we examined the effects of SFK inhibition on key regulators of mitochondrial function in HBE cells derived from IPF patients and controls. With respect to markers of mitochondrial biogenesis, the expression of the master regulator PGC1- $\alpha$ was decreased in HBE cells derived from IPF patients ( $p=0.0052$ ) (Figure 7.2A). PGC1- $\beta$ expression was only slightly but not significantly $(p=0.09)$ downregulated in HBE cells from IPF patients compared to those of controls while SFK inhibition restored this expression to significantly higher levels again (Figure 7.2B). No change in NRF1 expression could be observed between HBE cells from IPF patients and controls (Figure 7.2C) whereas NRF2 was significantly decreased in epithelial cells derived from IPF patients compared to control epithelial cells ( $p<0.001)$. After treatment with AZD, NRF2 expression in IPF derived HBE cells was upregulated again to control expression levels (Figure 7.2D). No difference in TFAM expression could be observed between control and IPF-derived epithelial cells $(p=0.99)$ (Figure 7.2E). Figure 7.2F depicts ERR $\alpha$ expression which was downregulated $(p=0.0037)$ and upregulated after AZD treatment $(p=0.0043)$ in IPF-derived HBE cells compared to those from controls. Both PPAR $\alpha$ and PPAR $\delta$ expression levels were downregulated in HBE cells from IPF patients compared to those of controls $(p=0.057$ and $p=0.0426)$ and unaffected by SFK inhibition (Figure $7.2 \mathrm{G}$ and $\mathrm{H}$ ). 
A

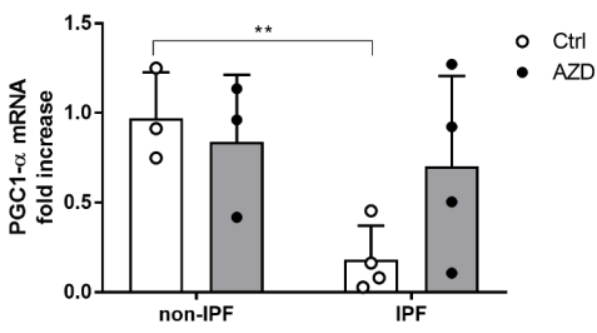

C

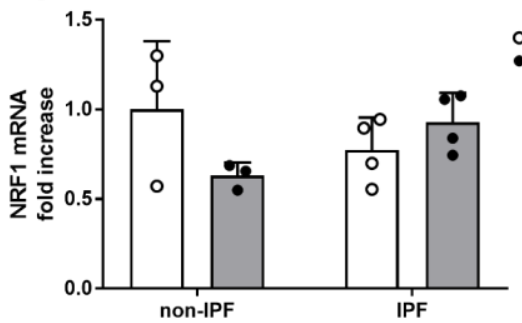

E

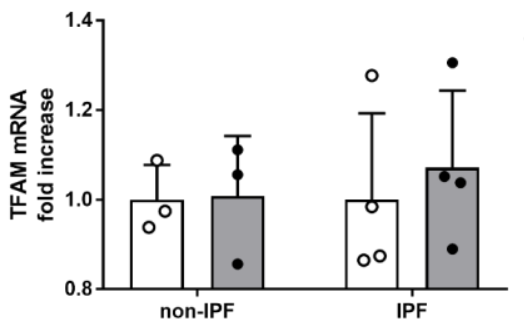

G

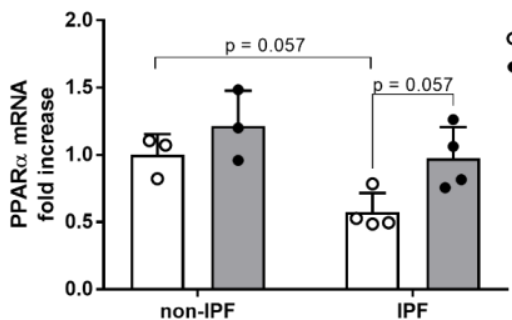

B

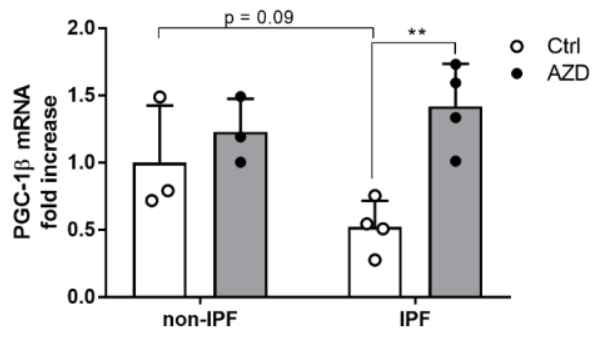

D

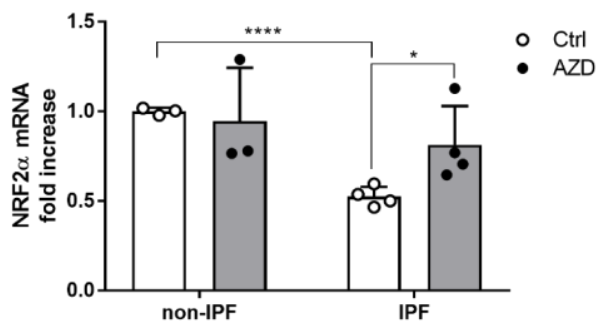

$\mathbf{F}$

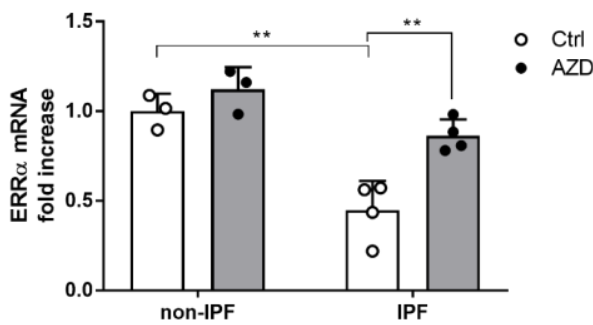

H

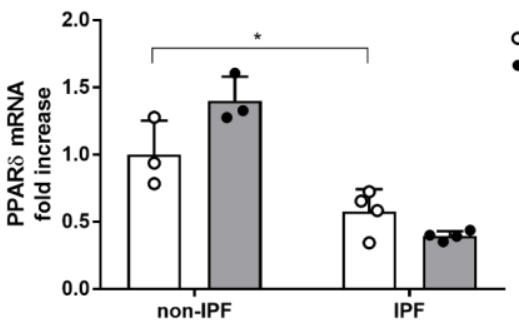

Figure 7.2: AZD increases expression levels of key regulators of mitochondrial biogenesis in IPF patients. Gene expression of (A) PGC1 $\alpha$ (\#), (B) PGC1 $\beta$ (\#), (C) NRF1 (\#\#), (D) NRF2 (\#), (E) TFAM (\#), (F) ERR $\alpha$ (\#), (G) PPAR $\alpha$ (\#), (H) PPARS (\#) and in the presence or absence of AZD $(1 \mu \mathrm{M})$ for 24 hrs in HBE cells derived from non-IPF $(n=3)$ and IPF patients $(n=4)$. Results are expressed as mean \pm SD. Variables were normal (\#) or non-normal (\#\#) distributed and significance of (non)parametric tests is depicted as $* P<0.05, * * P<0.01, * * * * P<0.0001$ 
Next, we assessed the mitophagy markers PINK1 and PARK2, which were slightly decreased in epithelial cells from IPF patients, although this decrease did not reach statistical significance ( $p=0.057$ and 0.056 respectively) (Figure 7.3A and B). SFK inhibition increased PINK1 expression in IPF-derived HBE cells $(p=0.02)$ but did not affect the expression of PARK2. The expression of BNIP, BNIP3L as well as FUNDC1, involved in receptor-mediated mitophagy, was not differentially affected in HBE cells derived from controls or IPF patients nor by the presence or absence of AZD (Figure 7.3C, D and E).

A

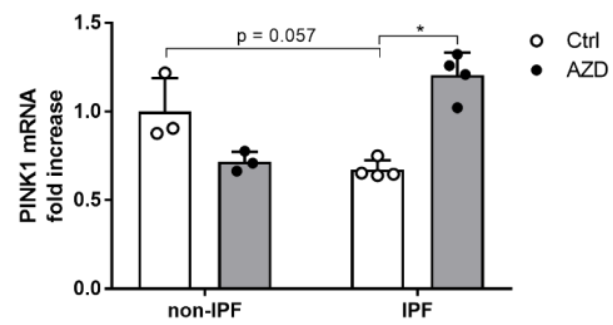

C

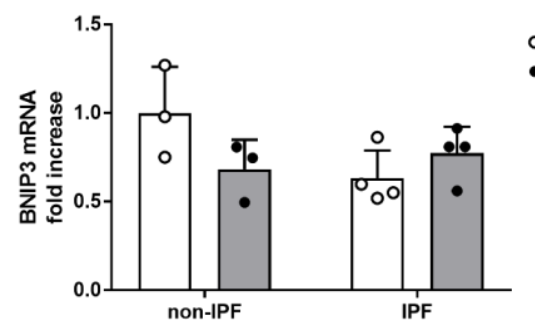

E

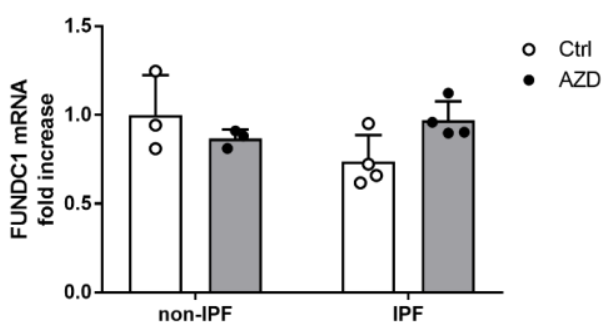

B

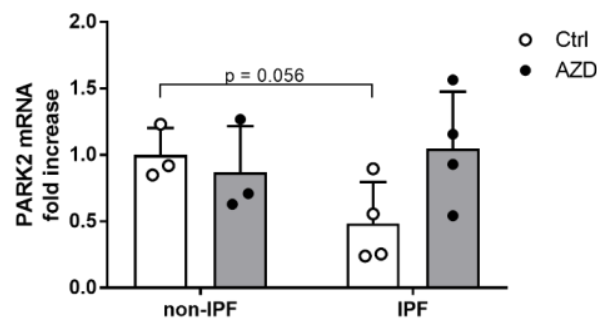

D

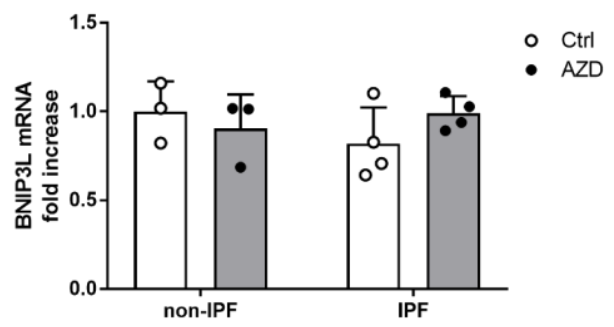

Figure 7.3: Expression of mitophagy-associated genes in HBE cells derived from non-IPF and IPF patients. Gene expression of (A) PINK1 (\#\#), (B) PARK2 (\#), (C) BNIP3 (\#), (D) BNIP3L (\#\#) and (E) FUNDC1 (\#) in the presence or absence of AZD $(1 \mu \mathrm{M})$ for 24 hrs in HBE cells derived from non-IPF $(n=3)$ and IPF patients $(n=4)$. Results are expressed as mean \pm SD. Variables were normal $d(\#)$ or non-normal (\#\#) distribute and significance of (non)parametric tests is depicted as ${ }^{*} \mathrm{P}<0.05$. 


\subsection{Inhibition of SFK reduces NOX4 expression}

In concordance with previous studies (32), epithelial cells from IPF patients have an increased expression of NOX4 compared to control HBE cells $(p=0.02)$ which was reduced upon SFK inhibition $(p=0.02)$ (Figure 7.4).

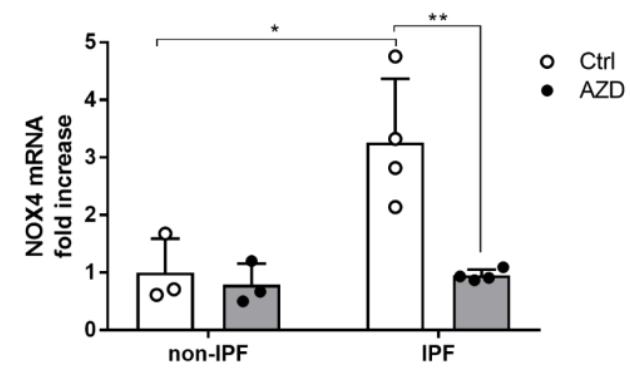

Figure 7.4: Expression of NOX4 in HBE cells derived from non-IPF and IPF patients. Gene expression in the presence or absence of AZD $(1 \mu M)$ for 24 hrs in HBE cells derived from non-IPF $(n=3)$ and IPF patients $(n=4)$. Results are expressed as mean $\pm \mathrm{SD}$. Variables were normal distributed and significance of parametric test is depicted as ${ }^{*} \mathrm{P}<0.05,{ }^{*} \mathrm{P}<0.01$.

\subsection{SFK are involved in cell damage and cell death}

When mitochondrial biogenesis decreases and mitophagy is impaired, dysfunctional mitochondria can accumulate leading to cellular apoptosis due to the impaired production of new mitochondria and decreased removal of dysfunctional mitochondria. As permeabilization of the plasma membrane is a key event in cell death, LDH release was determined. Epithelial cells from IPF patients have an increased LDH release compared to HBE cells from controls $(p=0.0043)$, which was prevented by AZD treatment $(p=0.007)$ (Figure 7.5A). As apoptosis can be regulated through the release of apoptotic proteins from the mitochondria, the next step was to evaluate the expression levels of various members of this intrinsic regulated apoptosis pathway. We found that HBE cells from IPF patients have an increased expression of the pro-apoptotic protein BAX (Figure 7.5B) which causes the additional release of pro-apoptotic proteins from the mitochondria and consequently the activation of caspases and cell death. Interestingly, these enhanced BAX levels in HBE cells from IPF patients were decreased again upon inhibition of SFK by AZD pretreatment (Figure 7.5B). No difference in the expression of the anti-apoptotic protein $\mathrm{BCL} 2$ was found between epithelial cells from IPF patients and controls irrespective of SFK inhibition (Figure 7.5C). 
A

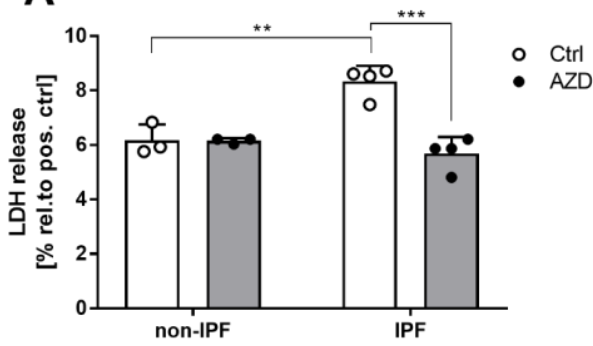

C

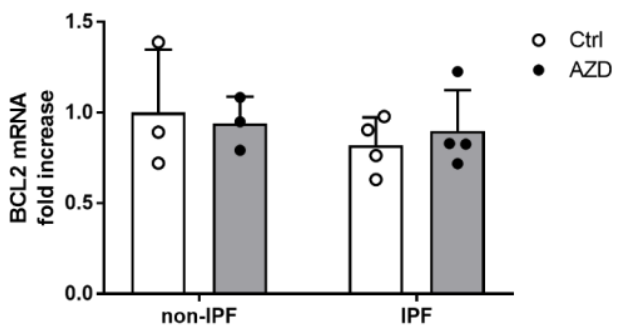

B

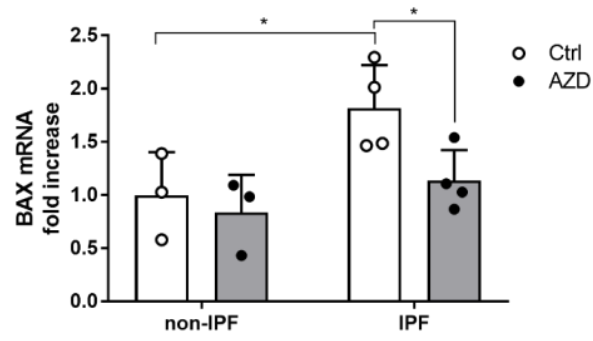

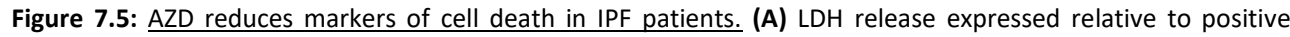
control. (B) Gene expression of BAX and (C) BCL-2 in the presence or absence of AZD (1 $\mu \mathrm{M})$ for $24 \mathrm{hrs}$ in HBE cells derived from non-IPF $(n=3)$ and IPF patients $(n=4)$. Results are expressed as mean \pm SD. Variables were normal distributed and significance of parametric test is depicted as $* P<0.05$. 


\section{Discussion}

The regulation of mitochondrial biogenesis and mitophagy is an important adaptive response of cells to cope with stress responses. Mitochondria from IPF patients are dysfunctional and contribute to enhanced apoptosis of AECs in response to injury to the lung epithelium (18).

Our results show that HBE cells from IPF patients have an increased activation of SFK which was accompanied by decreased expression levels of regulators of mitochondrial biogenesis and increased levels of mitophagy-associated genes. SFK inhibition restores the observed alterations in mitochondrial biogenesis and mitophagy, thereby increasing cell survival as shown by decreased release of LDH. Besides improving epithelial cell survival, our data imply that SFK inhibition also reduces NOX4 expression in IPF patients.

Currently, only two FDA-approved drugs are available for IPF patients, e.g. pirfenidone and nintedanib, which only demonstrate modest effects on the progression of the disease and are not effective in all IPF patients (33). Although the exact modes of action of both drugs are still unknown, the receptor tyrosine kinase inhibitor nintedanib (34) also inhibits nonreceptor tyrosine kinases such as the SRC kinase family (35). Pharmacological inhibitors such as PP2 and AZD that target SFK more specifically could serve as alternative treatment strategy for IPF as they have shown to attenuate pulmonary collagen deposition in a mouse model of bleomycin-induced pulmonary fibrosis $(4,5)$. Here we show that IPF patients have an increased activation of SFK. It has been demonstrated before that various growth factors, for instance transforming growth factor (TGF)- $\beta$ (36) and platelet-derived growth factor (PDGF) (5) enhance SFK activation in lung cells and fibroblasts respectively. TGF- $\beta 1$ as well PDGF have been implicated in the development of IPF $(37,38)$ and could therefore contribute to the increased activation of SFK.

Our data indicate that inhibition of SFK by AZD reduced cell damage and potentially cell death in HBE cells from patients with IPF, possibly through downregulation of the proapoptotic protein BAX. Additionally, AZD stimulation enhanced the expression of key regulators of mitochondrial biogenesis and mitophagy in IPF-derived HBE cells, which might play a role in increasing cell survival through the removal of dysfunctional mitochondria. Indeed, it has been shown before that the removal of damaged mitochondria through upregulation of mitophagy might improve epithelial cell survival (18).

Studies have indicated before that SFK including CSRC, FYN and LYN can reside in the mitochondria (12) and not only directly modulates the activity of the OXPHOS machine but can also regulate mitophagy $(39,40)$. Other studies suggest that CSRC phosphorylates cytochrome $c$ oxidase (COX) (41) as well as complex I, III, IV and V (42). When the OXPHOS machinery is impaired, less ATP is produced leading to energy deprivation, oxidative stress and eventually mitochondrial dysfunction (43). Interestingly, ATP is an allosteric inhibitor 
of NOX4 (44) and IPF patients have decreased levels of intracellular levels of ATP (45), indicating malfunctioning of mitochondria that might be linked to the increased NOX4 expression shown in this disease as well (46).

NOX4-dependent ROS production is crucial in mediating epithelial cell death in response to the profibrotic cytokine TGF- $\beta 1$ (32). It has been shown that TGF- $\beta$ mediated NOX4 expression is also regulated by mitochondrial ROS in lung fibroblasts from IPF patients (47) indicating the important role of mitochondria in NOX4 regulation. Interestingly, it was suggested that NOX4 might also be present in mitochondria as it contains a 73-amino acid long mitochondrial localization signal in its N-terminus (24). Furthermore, NOX4 has been implicated in the regulation of mitochondrial biogenesis and bioenergetics (25) as lung fibroblasts from NOX4 knockout mice display enhanced mitochondrial respiration and increased production of mitochondria which was mediated by TFAM as well as NRF2 but independent of PGC1- $\alpha$ (25). Furthermore, studies in human endothelial cells indicate that NOX4 can also directly interact with mtROS production through inhibition of mitochondrial complex I, thereby reducing the mitochondrial respiratory capacity and contributing to mitochondrial dysfunction (48), although the specific subcellular localization of NOX4 was not determined in this study. The effect of SFK inhibition on regulators of mitochondrial biogenesis and mitophagy could be mediated through the inhibition of NOX4 as NOX4 expression is dependent on SFK activation. It has been suggested before that the SFK member FYN and NOX4 colocalize in mitochondria and that FYN is phosphorylating NOX4 at tyrosine 566 in the C-terminus thereby regulating its activity (49). However, in this study FYN was negatively affecting the activity of NOX4, suggesting that a different mechanism or a different SFK member might be involved in our study as through inhibition of SFK, NOX4 expression was attenuated. Interestingly, it has been shown that SFK can phosphorylate the NOX co-activator p47 phox resulting in enhanced NOX activity in human endothelial cells (50) as well as in smooth muscle cells (51). Inhibition of SFK by PP2 attenuated activation of SFK as well as ROS production (50). This finding suggests that other cofactors, for instance $\mathrm{p} 22^{\text {phox }}$ which mediates the activation of NOX4 (52), may be phosphorylated as well. Indeed, p22 ${ }^{\text {phox }}$ can be phosphorylated at tyrosine residues which also correlated with NOX activation in neutrophils (53), however, the role of SFK in p22 ${ }^{\text {phox }}$ phosphorylation is unclear. It would be of interest to address the role of SFK inhibition on p22phox phosphorylation and the effects on NOX4 activity herein.

This study was associated with some limitations of which the very small sample size is the most prominent one, indicating that our results need to be repeated in a larger cohort in the future to draw definitive conclusions. Additionally, the localization of both residing and activated SFK in the mitochondria needs to be confirmed. It would also be of interest to identify specific SFK that are involved in the regulation of mitochondrial biogenesis and mitophagy. The observation that NOX4 expression is up in IPF and can be normalized by 
inhibiting SFK suggests that the effect these kinases have on mitochondrial homeostasis could be partly due to the involvement of NOX4. Consequently, new research should be focused on whether this increased NOX4 expression is accompanied by enhanced NOX4 activation and localization to the mitochondria. Moreover, it would be of interest to check if SFK inhibition would affect the activity of NOX4 in the mitochondria and thus affect mitochondrial homeostasis via the enzyme instead of directly via SFK.

In conclusion, this study shows for the first time that HBE cells from IPF patients display an increased activation of SFK kinases and that their inhibition attenuates NOX4 expression and reduces LDH release. This latter finding might be associated with increased mitochondrial turnover as SFK inhibition also increased expression of various regulators of mitochondrial biogenesis and mitophagy. 


\section{Acknowledgements}

CV greatly appreciates the research support from the Lung Foundation Netherlands (Project number: 9.2.17.214FE). The authors would like to thank H. Gosker for his input. 


\section{References}

1. Ley B, Collard HR, King TE, Jr. Clinical course and prediction of survival in idiopathic pulmonary fibrosis. Am J Respir Crit Care Med. 2011;183(4):431-40.

2. Selman M, Pardo A. Revealing the pathogenic and aging-related mechanisms of the enigmatic idiopathic pulmonary fibrosis. an integral model. Am J Respir Crit Care Med.

2014;189(10):1161-72.

3. Selman M, Pardo A. Role of epithelial cells in idiopathic pulmonary fibrosis: from innocent targets to serial killers. Proc Am Thorac Soc. 2006;3(4):364-72.

4. Hu M, Che P, Han X, Cai GQ, Liu G, Antony V, et al. Therapeutic targeting of SRC kinase in myofibroblast differentiation and pulmonary fibrosis. The Journal of pharmacology and experimental therapeutics. 2014;351(1):87-95.

5. Lu YY, Zhao XK, Yu L, Qi F, Zhai B, Gao CQ, et al. Interaction of Src and Alpha-V Integrin Regulates Fibroblast Migration and Modulates Lung Fibrosis in A Preclinical Model of Lung Fibrosis. Sci Rep. 2017;7:46357.

6. Roskoski R, Jr. Src protein-tyrosine kinase structure and regulation. Biochem Biophys Res Commun. 2004;324(4):1155-64.

7. Thomas SM, Brugge JS. Cellular functions regulated by Src family kinases. Annu Rev Cell Dev Biol. 1997;13:513-609.

8. Roskoski R, Jr. Src kinase regulation by phosphorylation and dephosphorylation. Biochem Biophys Res Commun. 2005;331(1):1-14.

9. Heppner DE, Dustin CM, Liao C, Hristova M, Veith C, Little AC, et al. Direct cysteine sulfenylation drives activation of the Src kinase. Nat Commun. 2018;9(1):4522.

10. Giannoni E, Taddei ML, Chiarugi P. Src redox regulation: again in the front line. Free Radic Biol Med. 2010;49(4):516-27.

11. Koc EC, Miller-Lee JL, Koc H. Fyn kinase regulates translation in mammalian mitochondria. Biochim Biophys Acta Gen Subj. 2017;1861(3):533-40.

12. Salvi M, Brunati AM, Bordin L, La Rocca N, Clari G, Toninello A. Characterization and location of Src-dependent tyrosine phosphorylation in rat brain mitochondria. Biochim Biophys Acta. 2002;1589(2):181-95.

13. Salvi M, Brunati AM, Toninello A. Tyrosine phosphorylation in mitochondria: a new frontier in mitochondrial signaling. Free Radic Biol Med. 2005;38(10):1267-77.

14. Cesaro L, Salvi M. Mitochondrial tyrosine phosphoproteome: new insights from an up-todate analysis. Biofactors. 2010;36(6):437-50.

15. Jornayvaz FR, Shulman Gl. Regulation of mitochondrial biogenesis. Essays Biochem. 2010;47:69-84.

16. Giguere V. Transcriptional control of energy homeostasis by the estrogen-related receptors. Endocr Rev. 2008;29(6):677-96.

17. Tsubouchi K, Araya J, Kuwano K. PINK1-PARK2-mediated mitophagy in COPD and IPF pathogeneses. Inflamm Regen. 2018;38:18.

18. Bueno M, Lai YC, Romero Y, Brands J, St Croix CM, Kamga C, et al. PINK1 deficiency impairs mitochondrial homeostasis and promotes lung fibrosis. J Clin Invest. 2015;125(2):521-38.

19. Patel AS, Song JW, Chu SG, Mizumura K, Osorio JC, Shi Y, et al. Epithelial cell mitochondrial dysfunction and PINK1 are induced by transforming growth factor-beta1 in pulmonary fibrosis. PloS one. 2015;10(3):e0121246.

20. Ney PA. Mitochondrial autophagy: Origins, significance, and role of BNIP3 and NIX. Biochim Biophys Acta. 2015;1853(10 Pt B):2775-83.

21. Sureshbabu A, Bhandari V. Targeting mitochondrial dysfunction in lung diseases: emphasis on mitophagy. Front Physiol. 2013;4:384. 
22. Burlacu A. Regulation of apoptosis by Bcl-2 family proteins. J Cell Mol Med. 2003;7(3):249-

57.

23. Tait SW, Green DR. Mitochondria and cell death: outer membrane permeabilization and beyond. Nat Rev Mol Cell Biol. 2010;11(9):621-32.

24. Graham KA, Kulawiec M, Owens KM, Li X, Desouki MM, Chandra D, et al. NADPH oxidase 4 is an oncoprotein localized to mitochondria. Cancer Biol Ther. 2010;10(3):223-31.

25. Bernard K, Logsdon NJ, Miguel V, Benavides GA, Zhang J, Carter AB, et al. NADPH Oxidase 4 (Nox4) Suppresses Mitochondrial Biogenesis and Bioenergetics in Lung Fibroblasts via a Nuclear Factor Erythroid-derived 2-like 2 (Nrf2)-dependent Pathway. J Biol Chem. 2017;292(7):3029-38.

26. Wang $\mathrm{Y}$, Hekimi S. Mitochondrial dysfunction and longevity in animals: Untangling the knot. Science. 2015;350(6265):1204-7.

27. Kahn N, Kuner R, Eberhardt R, Meister M, Muley T, Winteroll S, et al. Gene expression analysis of endobronchial epithelial lining fluid in the evaluation of indeterminate pulmonary nodules. J Thorac Cardiovasc Surg. 2009;138(2):474-9.

28. Raghu G, Collard HR, Egan JJ, Martinez FJ, Behr J, Brown KK, et al. An official ATS/ERS/JRS/ALAT statement: idiopathic pulmonary fibrosis: evidence-based guidelines for diagnosis and management. Am J Respir Crit Care Med. 2011;183(6):788-824.

29. Wu R. Growth of human lung tumor cells in culture. Culture of Human Tumor Cells Pfragner R, Freshney RI (eds) Wiley-Liss Inc: Hoboken, NJ. 2004:1-21.

30. Tibaldi E, Brunati AM, Massimino ML, Stringaro A, Colone M, Agostinelli E, et al. SrcTyrosine kinases are major agents in mitochondrial tyrosine phosphorylation. J Cell Biochem. 2008;104(3):840-9.

31. Lim S, Smith KR, Lim ST, Tian R, Lu J, Tan M. Regulation of mitochondrial functions by protein phosphorylation and dephosphorylation. Cell Biosci. 2016;6:25.

32. Carnesecchi S, Deffert C, Donati Y, Basset O, Hinz B, Preynat-Seauve O, et al. A key role for NOX4 in epithelial cell death during development of lung fibrosis. Antioxidants \& redox signaling. 2011;15(3):607-19.

33. Raghu G, Selman M. Nintedanib and pirfenidone. New antifibrotic treatments indicated for idiopathic pulmonary fibrosis offer hopes and raises questions. Am J Respir Crit Care Med. 2015;191(3):252-4.

34. Hostettler KE, Zhong J, Papakonstantinou E, Karakiulakis G, Tamm M, Seidel P, et al. Antifibrotic effects of nintedanib in lung fibroblasts derived from patients with idiopathic pulmonary fibrosis. Respir Res. 2014;15:157.

35. Hilberg F, Roth GJ, Krssak M, Kautschitsch S, Sommergruber W, Tontsch-Grunt U, et al. BIBF 1120: triple angiokinase inhibitor with sustained receptor blockade and good antitumor efficacy. Cancer Res. 2008;68(12):4774-82.

36. Zhang H, Davies KJ, Forman HJ. TGFbeta1 rapidly activates Src through a non-canonical redox signaling mechanism. Arch Biochem Biophys. 2015;568:1-7.

37. Fernandez IE, Eickelberg $O$. The impact of TGF-beta on lung fibrosis: from targeting to biomarkers. Proc Am Thorac Soc. 2012;9(3):111-6.

38. Wollin L, Wex E, Pautsch A, Schnapp G, Hostettler KE, Stowasser S, et al. Mode of action of nintedanib in the treatment of idiopathic pulmonary fibrosis. Eur Respir J. 2015;45(5):1434-45.

39. Liu L, Feng D, Chen G, Chen M, Zheng $Q$, Song P, et al. Mitochondrial outer-membrane protein FUNDC1 mediates hypoxia-induced mitophagy in mammalian cells. Nat Cell Biol. 2012;14(2):177-85.

40. Chen $\mathrm{G}$, Han Z, Feng D, Chen $\mathrm{Y}$, Chen $\mathrm{L}, \mathrm{Wu} \mathrm{H}$, et al. A regulatory signaling loop comprising the PGAM5 phosphatase and CK2 controls receptor-mediated mitophagy. Mol Cell. 2014;54(3):36277.

41. Miyazaki T, Neff L, Tanaka S, Horne WC, Baron R. Regulation of cytochrome c oxidase activity by c-Src in osteoclasts. J Cell Biol. 2003;160(5):709-18. 
42. Hebert Chatelain E, Dupuy JW, Letellier T, Dachary-Prigent J. Functional impact of PTP1Bmediated Src regulation on oxidative phosphorylation in rat brain mitochondria. Cell Mol Life Sci. 2011;68(15):2603-13.

43. Lemasters JJ, Qian T, Bradham CA, Brenner DA, Cascio WE, Trost LC, et al. Mitochondrial dysfunction in the pathogenesis of necrotic and apoptotic cell death. J Bioenerg Biomembr. 1999;31(4):305-19.

44. Shanmugasundaram K, Nayak BK, Friedrichs WE, Kaushik D, Rodriguez R, Block K. NOX4 functions as a mitochondrial energetic sensor coupling cancer metabolic reprogramming to drug resistance. Nat Commun. 2017;8(1):997.

45. Kang YP, Lee SB, Lee JM, Kim HM, Hong JY, Lee WJ, et al. Metabolic Profiling Regarding Pathogenesis of Idiopathic Pulmonary Fibrosis. J Proteome Res. 2016;15(5):1717-24.

46. Hecker L, Vittal R, Jones T, Jagirdar R, Luckhardt TR, Horowitz JC, et al. NADPH oxidase-4 mediates myofibroblast activation and fibrogenic responses to lung injury. Nat Med. 2009;15(9):1077-81.

47. Jain M, Rivera S, Monclus EA, Synenki L, Zirk A, Eisenbart J, et al. Mitochondrial reactive oxygen species regulate transforming growth factor-beta signaling. J Biol Chem. 2013;288(2):770-7. 48. Koziel R, Pircher H, Kratochwil M, Lener B, Hermann M, Dencher NA, et al. Mitochondrial respiratory chain complex I is inactivated by NADPH oxidase Nox4. Biochem J. 2013;452(2):231-9. 49. Matsushima S, Kuroda J, Zhai P, Liu T, Ikeda S, Nagarajan N, et al. Tyrosine kinase FYN negatively regulates NOX4 in cardiac remodeling. J Clin Invest. 2016;126(9):3403-16.

50. Chowdhury AK, Watkins T, Parinandi NL, Saatian B, Kleinberg ME, Usatyuk PV, et al. Srcmediated tyrosine phosphorylation of p47phox in hyperoxia-induced activation of NADPH oxidase and generation of reactive oxygen species in lung endothelial cells. J Biol Chem. 2005;280(21):20700-11.

51. Touyz RM, Yao G, Schiffrin EL. c-Src induces phosphorylation and translocation of p47phox: role in superoxide generation by angiotensin II in human vascular smooth muscle cells. Arterioscler Thromb Vasc Biol. 2003;23(6):981-7.

52. Martyn KD, Frederick LM, von Loehneysen K, Dinauer MC, Knaus UG. Functional analysis of Nox4 reveals unique characteristics compared to other NADPH oxidases. Cell Signal. 2006;18(1):6982.

53. Regier DS, Greene DG, Sergeant S, Jesaitis AJ, McPhail LC. Phosphorylation of p22phox is mediated by phospholipase D-dependent and -independent mechanisms. Correlation of NADPH oxidase activity and p22phox phosphorylation. J Biol Chem. 2000;275(37):28406-12. 




\section{Chapter 8}

\section{Differences in treatment responses in bronchial epithelial cells from Idiopathic Pulmonary Fibrosis (IPF) patients: a first step towards personalized medicine?}

Carmen Veith, Agnes W. Boots, Marie-José Drittij, Michael Kreuter, Marc Schneider, Michael Meister and Nicolas Kahn.

Pilot study 


\section{Abstract}

Rationale: Idiopathic pulmonary fibrosis (IPF) is a chronic progressive interstitial lung disease with a detrimental prognosis. While antifibrotic therapies ameliorate the course of the disease in IPF, this disease is still uncurable. Moreover, individual responses to antifibrotics varies substantially. IPF is mainly driven by continuous epithelial injuries, associated with oxidative stress, profibrotic processes and inflammation. Additionally, SRC family kinases (SFK) contribute to fibrotic remodeling, although the effect of SFK inhibition on IPF progression is not known. Aim of our study was to differentiate bronchial epithelial cells (HBE) into a complete bronchial epithelium and to evaluate the individual responses towards pirfenidone, nintedanib and SFK inhibitor saracatinib on markers of the redox and inflammatory status herein.

Methods: In this pilot study, HBE cells were collected from epithelial lining fluid (ELF) from IPF patients $(n=4)$ and controls $(n=3)$ by bronchoscopic microsampling (BMS) from subsegmental bronchi. HBE cells were then expanded and differentiated into a complete bronchial epithelium in which NOX4 expression, antioxidant gene expression and proinflammatory cytokine secretion were evaluated in the absence or presence of pirfenidone, nintedanib or SFK inhibitor saracatinib.

Results: HBE cells from ELF differentiated into a complete bronchial epithelium consisted of ciliated epithelial, basal, goblet and club cells. NOX4 expression was slightly increased in HBE cells from IPF patients compared to control epithelial cells but differed on individual level. In patients with higher NOX4 expression, pirfenidone induced antioxidant genes such as NRF2, HO-1, yGCS, SOD1, SOD2 and CAT. NOX4 expression was decreased by treating HBE cells from IPF patients with nintedanib $(p=0.07)$. Furthermore, IL-6 ( $p=0.09)$ and IL-8 secretion $(p=0.014)$ were increased in HBE cells derived from IPF patients and treatment with saracatinib reduced this elevated IL-8 secretion significantly $(p=0.02)$.

Conclusion: Our results show that responsiveness to treatment may vary substantially between individual IPF patients, thereby indicating that IPF patients might benefit from personalized treatment.

Keywords: IPF, primary bronchial epithelial cells, nintedanib, pirfenidone, saracatinib, personalized medicine 


\section{Introduction}

Idiopathic pulmonary fibrosis (IPF) is a progressive and irreversible lung disease of unknown etiology, characterized by scarring of the lung tissue which causes symptoms such as non-productive cough and breathlessness. IPF has a median survival of only 3.8 years (1) and affects approximately 5 million people worldwide although its overall incidence is increasing $(2,3)$. Next to the exposure to environmental factors, e.g. cigarette smoke and asbestos, aging is the most well-known risk factor as can also be deducted from the fact that two thirds of IPF patients are older than 60 years at time of diagnosis (4, 5). The pathogenic mechanisms underlying IPF are still mainly unclear, but the current paradigm is that a disrupted homeostasis of epithelial cells upon damage by various triggers $(6,7)$ plays an important role in the development of the disease. Recurrent epithelial injury leads to aberrant wound healing responses and eventually causes the remodeling of the lung structure by promoting epithelial cell apoptosis and excessive collagen deposition in the alveolar space (6). Furthermore, IPF is associated with an increased oxidant burden and diminished expression of antioxidants and detoxification enzymes, including heme oxygenase-1 (HO-1), superoxide dismutase (SOD), catalase (CAT) and glutamate cysteine synthase ( $\gamma \mathrm{GCS}$, the rate-limiting enzyme in glutathione synthesis) (8-13) which is thought to further enhance epithelial injury. An important source of reactive oxygen species (ROS) in the lungs is the family of NADPH oxidases (NOXes). The family member NOX4 is highly upregulated in the lungs of IPF patients (14) and expressed by epithelial cells as well as (myo)fibroblasts promoting alveolar epithelial cell (AEC) death, (myo)fibroblast differentiation and collagen deposition (15).

Recently, the antifibrotic drugs pirfenidone and nintedanib were shown to ameliorate the course of the disease by slowing down the progression of the disease (16). Yet, there is still no cure or reversibility of the disease Pirfenidone is suggested to have antioxidative, anti-inflammatory and antifibrotic effects although its exact working mechanisms and targets remain to be elucidated $(16,17)$. In vitro and in bleomycin-induced murine pulmonary fibrosis, pirfenidone mainly reduces markers of oxidative stress $(18,19)$, decreases the secretion of pro-inflammatory cytokines (e.g. TNF- $\alpha$, IL-1 $\beta$, IL-6) (20) and inhibits the proliferation of fibroblasts as well as their differentiation into myofibroblasts thereby reducing secretion of collagen (21). Nintedanib, originally developed as an anticancer drug, is an intracellular receptor tyrosine kinase inhibitor which mainly inhibits the platelet-derived growth factor receptor (PDGFR), fibroblast growth factor receptor (FGFR) and vascular growth factor receptor (VEGFR) (16). Nintedanib possesses antifibrotic effects as it inhibits the actions of fibroblasts (22), but its exact working mechanisms still remain to be elucidated as well. Additionally, the effect of both of these drugs on the lung epithelium is not clear yet as most studies thus far have investigated their influences only on (myo)fibroblasts $(21,23-25)$. Recently, a study has demonstrated 
that low concentrations of pirfenidone $(250 \mu \mathrm{M})$ and nintedanib $(1 \mu \mathrm{M})$ also reduce fibrotic gene expression in isolated murine alveolar epithelial cells (26). This little knowledge regarding their mode of action, combined with the fact that IPF is a very heterogeneous disease, makes it very difficult to predict the effects of either pirfenidone or nintedanib in individual patients.

Recently, it has been suggested that the non-receptor tyrosine inhibitor saracatinib (AZD0530, AstraZeneca), which inhibits SRC family kinases (SFK) and BCR-ABL kinases, could be a potential treatment strategy in IPF (27). SFK are involved in various signaling pathways important for cellular homeostasis, including cell differentiation and proliferation $(28,29)$, pathways that are often dysregulated in IPF $(30)$. It has been shown that SFK are activated in response to TGF- $\beta$ and inhibiting SRC kinases by AZD0530 in an experimental model of pulmonary fibrosis reduced the fibrotic area as well as collagen deposition in the lung $(27,31)$.

The paradigm regarding IPF pathology has shifted and the disease is now considered an epithelial-driven and fibroblast-activated process with mild inflammation (32). Therefore, to effectively treat IPF not only fibroblasts but also epithelial cells need to be targeted (33) which makes it pivotal to investigate the effects of antifibrotic drugs on the lung epithelium as well. This pilot study was designed to setup methodology for a larger scale study, ultimately designed to identify predictors for treatment efficiency. More specifically, we evaluated the antioxidative and anti-inflammatory effects of SFK inhibitor AZD0530 in differentiated bronchial epithelial cells (HBE) from IPF patients and compare them to those of pirfenidone and nintedanib. To this end, primary HBE cells isolated from non-treated IPF patients were treated with pirfenidone, nintedanib or saracatinib after which NOX4 expression, antioxidant gene expression and pro-inflammatory cytokine secretion were evaluated. 


\section{Material and Methods}

\subsection{Bronchoscopic microsampling}

Human bronchial epithelial (HBE) cells were obtained from endobronchial lining fluid (ELF) of IPF patients and controls by minimally invasive bronchoscopic microsampling (BMS) during bronchoscopy from subsegmental airways as previously described (34). All participants gave written informed consent, and the study was approved by the ethics committee of the University of Heidelberg, Germany.

\subsection{Patient's characteristics}

In total 7 patients were enrolled in this study via recruitment at the Center for interstitial and rare lung diseases, Thoraxklinik, University of Heidelberg (see Table 8.1 for their characteristics). All patients gave their written informed consent.

\section{Lung fibrosis patients}

IPF was diagnosed according to the ATS-ERS consensus criteria (35) in 4 male patients. Two were ex-smokers, 1 never smoked and 1 was an active smoker. At the time of sampling, none of the patient received treatment yet.

\section{Control patients (non-ILD)}

For the control group, 3 patients ( 2 males, 1 female) undergoing bronchoscopy for further investigation of indeterminate pulmonary nodules were included of whom the main characteristics are shown in Table 8.1. ELF was obtained from a noninvolved segment, from the contralateral lung, opposite the solitary lesion to minimize possible influences of the suspected malignancy. Two patients were smokers and 1 was an ex-smoker.

Table 8.1: Patient characteristics. Age is expressed in years, DLCO (diffuse capacity of the lung for carbon monoxide), $\mathrm{FEV}_{1}$ (forced expiratory volume in $1 \mathrm{~s}$ ) and FVC (forced vital capacity) in percentage of the predicted value based on age and gender. Data are expressed as range (mean $\pm S D) .\left({ }^{*} P<0.05,{ }^{* * *} P<0.001\right)$

\begin{tabular}{|c|c|c|}
\hline & $\begin{array}{l}\text { IPF patients } \\
(n=4)\end{array}$ & $\begin{array}{c}\text { Controls, non-IPF } \\
\text { patients }(n=3)\end{array}$ \\
\hline Gender $(\mathrm{m} / \mathrm{f})$ & $4(4 / 0)$ & $3(2 / 1)$ \\
\hline Age (years) & $67-73(72 \pm 3.9) * * *$ & $53-55(54 \pm 1.2)$ \\
\hline DLCO (\% predicted) & $\begin{array}{c}\text { 36.5-48.4 (41 } \pm 5.3) \\
* * *\end{array}$ & $81.9-101.8(94 \pm 11)$ \\
\hline FVC (\% predicted) & $60.9-89.7(80 \pm 14) *$ & $93-113.3(106 \pm 12)$ \\
\hline $\begin{array}{l}\text { Smoking status } \\
\text { (never, ex, current) }\end{array}$ & $1,2,1$ & $0,2,1$ \\
\hline
\end{tabular}




\subsection{Cell culturing and cell treatment}

Primary HBE cells were centrifuged for $5 \mathrm{~min}$ at room temperature and $300 \mathrm{x}$ g to elute sampled cells from sponges. Afterwards, cells were resuspended and expanded in DMEM/F12 media (Gibco, Carlsbad, CA) supplemented with bovine pituitary extract $(0.004 \mathrm{~mL} / \mathrm{mL})$, epidermal growth factor $(10 \mathrm{ng} / \mathrm{mL})$, insulin $(5 \mu \mathrm{g} / \mathrm{mL})$, hydrocortisone $(0.5$ $\mu \mathrm{g} / \mathrm{mL})$, triiodo-L-thyronine $(6.7 \mathrm{ng} / \mathrm{mL})$ and transferrin $(10 \mu \mathrm{g} / \mathrm{mL})$ (PromoCell, Heidelberg, Germany), sodium selenite (30 nM, Sigma), ethanolamine (10 $\mu \mathrm{M}$, Sigma), phosphorylethanolamine (10 $\mu \mathrm{M}$, Sigma), sodium pyruvate $(0.5 \mu \mathrm{M}, \mathrm{Gibco})$, adenine $(0.18$ $\mathrm{mM}$, Sigma), hepes (15 mM, Gibco), 1x GlutaMAX (Gibco) and in the presence of $10 \mu \mathrm{M}$ Rock-inhibitor (StemCell, Cologne, Germany), as described previously (36). After reaching confluency, cells were treansferred to 12 -well plates and plated at 90.000 cells/insert (ThinCert ${ }^{\mathrm{TM}}, 0.4 \mu \mathrm{m}$ pores, Greiner BioOne, Frickenhausen). After 2-3 days, cells were airlifted by removing the medium from the apical chamber and PneumaCult-ALI media (StemCell, Cologne, Germany) was added to the basal chamber only. Differentiation into a pseudostratified mucociliary epithelium was achieved after approximately 24-27 days. At day 28 , cells were stimulated with different concentrations of pirfenidone $(1 \mathrm{mM}, 500 \mu \mathrm{M}$, $100 \mu \mathrm{M})$, nintedanib $(1 \mu \mathrm{M}, 0.1 \mu \mathrm{M}, 0.01 \mu \mathrm{M})$ or saracatinib $(10 \mu \mathrm{M}, 1 \mu \mathrm{M}, 0.1 \mu \mathrm{M})$ (all Selleck Chemicals, Houston, TX) for 24 hrs after which RNA, proteins and supernatants were collected (see Figure 8.1). 


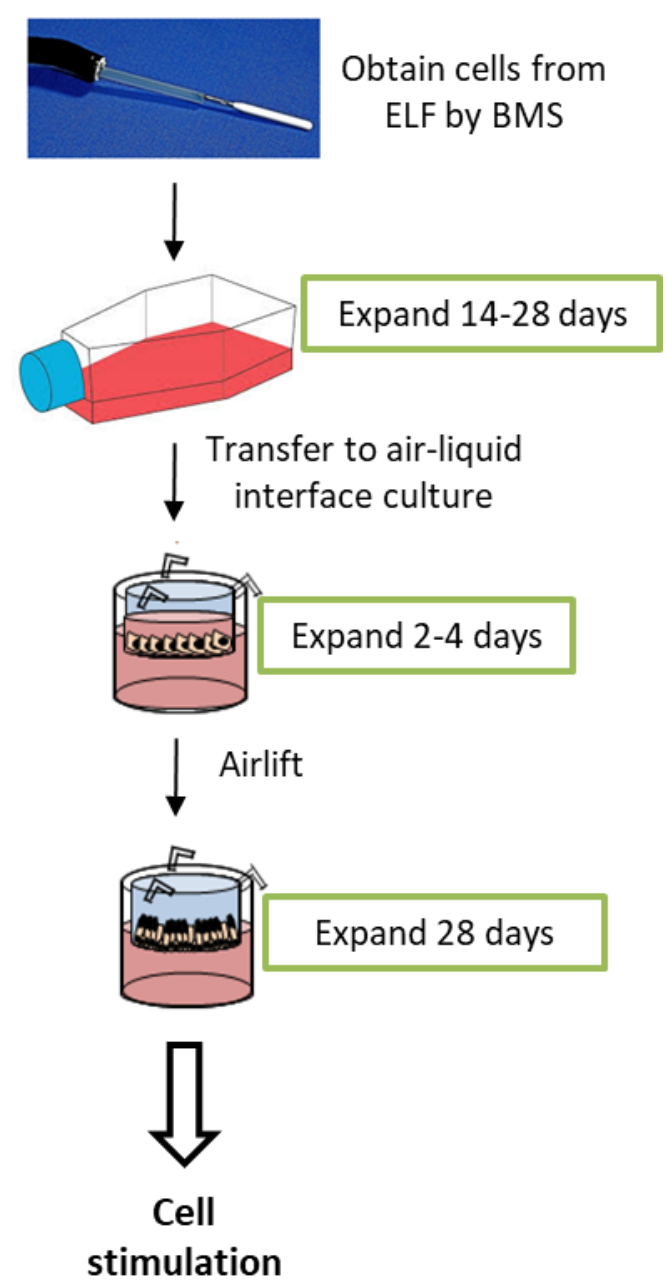

Figure 8.1: Culture method of HBE obtained by BMS. HBE cells were obtained from ELF by BMS and afterwards expanded in submerged cultures. After approximately 14-28 days, cells were transferred to an air-liquid interface culture in a submerged condition. After reaching confluency after 2-4 days, cells were airlifted and maintained until a pseudostratified epithelium has developed. At day 28, cells were stimulated with pirfenidone, nintedanib or saracatinib and RNA, proteins and supernatants were collected.

\subsection{Confocal imaging}

To validate differentiation of the pseudostratified mucociliary epithelium, cells were stained with specific antibodies 28 days after plating on inserts. Treated cells on inserts were washed with PBS, fixed on slides with $4 \%$ paraformaldehyde for $20 \mathrm{~min}$ at $4^{\circ} \mathrm{C}$. Afterwards, mounted slides were rinsed with PBS and incubated in PBS containing $0.2 \%$ Triton X-100 for $2 \mathrm{~min}$. Slides were then cut in 4 pieces and washed with PBS, blocked in PBS containing $1 \%$ bovine serum albumin (BSA) for 30 minutes and stained with 
antibodies targeted against keratin 5 (1:50, \#HPA059479, Sigma-Aldrich, St. Louis, Missouri, U.S.), tubulin- $\beta 4$ (1:100, \#T7941, Sigma-Aldrich), CC10 (1:300, \#RD18102222001, BioVendor, Heidelberg, Germany) and mucin 5B (1:300, abcam, \#ab3649, Berlin, Germany) overnight at $4{ }^{\circ} \mathrm{C}$. The next day, slides were washed with PBS and blocked for 10 $\min$ in PBS/1 \% BSA. Alexa Fluor secondary antibodies 488 and 594 (1:300, Invitrogen, Carlsbad, California, U.S.) were added and slides were incubated for $45 \mathrm{~min}$ at $37^{\circ} \mathrm{C}$ followed by Hoechst staining (1:10000, \#H1399, Invitrogen) for $10 \mathrm{~min}$ at room temperature. Images were obtained with confocal microscopy (Leica TCS SP5) and Leica Application Suite $\mathrm{X}$ software.

\subsection{ELISA}

Secretion of interleukin (IL)-8 and IL- 6 in cell culture supernatants was determined using human ELISA DuoSet kits (R\&D Systems, Minneapolis, MN) according to the manufacturer's instructions.

\subsection{LDH assay}

LDH cell death assay was used according to the instructions of the manufacturer (Thermo Scientific, Waltham, MA).

\subsection{RNA isolation and RT-PCR}

RNA was isolated and purified using the RNeasy mini kit (Qiagen, Venlo, the Netherlands) according to the manufacturer's instructions. The RNA concentration was determined using a Nanodrop spectrophotometer (Thermo Scientific) after which cDNA was synthesized from 500 ng isolated RNA using IScript (Biorad, Hercules, CA) according to the manufacturer's instructions. RT-PCR was performed using SYBR Green PCR Supermix (BioRad) with $4.4 \mu \mathrm{L}$ of 50 times diluted CDNA and $0.5 \mu \mathrm{mol} / \mathrm{L}$ predesigned primers. PCR amplifications were carried out for up to 55 cycles of denaturation $\left(95^{\circ} \mathrm{C}, 10 \mathrm{sec}\right.$ ) and annealing/elongation $\left(60^{\circ} \mathrm{C}, 60 \mathrm{sec}\right)$ for selected genes (Table 8.2). The gene expression was normalized to the house keeping gene $\beta$-actin and quantified according to the $2^{-\Delta \Delta C t}$ method to relatively quantify the expression of genes of interest. These genes include the ROS-producing enzyme NOX4 as well as a selection of important endogenous antioxidants (Table 8.2). 
Table 8.2: $\underline{\text { Human RT-PCR forward and reverse primer sequences. }}$

\begin{tabular}{|l|l|l|}
\hline Gene of interest & Forward primer sequence & Reverse primer sequence \\
\hline Actin & CCTGGCACCCAGCACAAT & GCCGATCCACACGGAGTACT \\
\hline NOX4 & TGGCAAGAGAACAGACCTGA & TGGGTCCACAACAGAAAACA \\
\hline Nrf2 & ACACGGTCCACAGCTCATC & TCTTGCCTCCAAAGTATGTCAA \\
\hline HO-1 & CTTCTTCACCTTCCCCAACA & GCTCTGGTCCTTGGTGTCAT \\
\hline YGCS & CGACCAATGGAGGTGCAGTTA & ACCCTAGTGAGCAGTACCACGAA \\
\hline CAT & GATGTGCATGCAGGACAATCAG & GCTTCTCAGCATTGTACTTGTCC \\
\hline SOD1 & CCACACCTTCACTGGTCCAT & CTAGCGAGTTATGGCGACG \\
\hline SOD2 & TGGACAAACCTCAGCCCTAACG & TGATGGCTTCCAGCAACTCCC \\
\hline
\end{tabular}

\subsection{Protein extraction and Western blotting}

Cell lysates were prepared using RIPA buffer (Thermo Scientific) containing $1 x$ phosphatase and protease inhibitors (Thermo Scientific) and $1 \mathrm{mmol} / \mathrm{L}$ phenylmethylsulfonyl fluoride (PMSF). Protein concentration was measured with BCA protein assay kit (Thermo Scientific). Aliquots of cell lysates containing $20 \mu \mathrm{g}$ proteins were separated on 10\% SDS-PAGE gels (Invitrogen), transferred to nitrocellulose membranes and probed with antibodies against NOX4 (1:1000) or $\beta$-ACTIN (1:5000; all from Cell Signaling, Danvers, MA). Primary antibodies were probed with rabbit or mouse respectively (Cell Signaling, Danvers, MA) and detected by means of chemiluminescence with SuperSignal ${ }^{\mathrm{TM}}$ West Pico PLUS or SuperSignal ${ }^{\mathrm{TM}}$ West Femto Maximum Sensitivity Substrate (Thermo Scientific) using Amersham Imager 600 (GE Healthcare Life Sciences, Hillerød, Iceland). Integrated density of pixels in each membrane was quantified using ImageJ software 1.47v (W. Rasband, National Institutes of Health, Bethesda, MD).

\subsection{Statistical Analysis}

All quantitative data are represented as means \pm SEM. Statistical differences between groups were evaluated by means of 1-ANOVA analysis corrected with Bonferroni's postanalysis or by means of Student's t-test in GraphPad Prism software (version 7.3; GraphPad Software, La Jolla, CA) and considered significant at a $P$ value less than 0.05 . 


\section{Results}

Human bronchial epithelial (HBE) cells were isolated from endobronchial lining fluid (ELF) of IPF patients and controls by bronchoscopic microsampling and grown in an air-liquidinterphase culture. After 28 days of expansion, a complete bronchial airway epithelium consisting of ciliated epithelial, goblet, club and basal cells has developed. HBE cells were stained with specific antibodies and confocal microscopy was performed showing this successful differentiation of BMS-derived cultures (Figure 8.2). The keratin 5 staining, indicating basal cells, appeared mostly at the bottom of the culture whereas tubulin- $\beta 4$ staining, indicating ciliated epithelial cells, was distributed on the top of the cells (Figure 8.2A and C). The CC10 (Club cells) and mucin 5B staining were evenly distributed throughout the culture (Figure 8.2B and $\mathrm{D}$ ). 
A

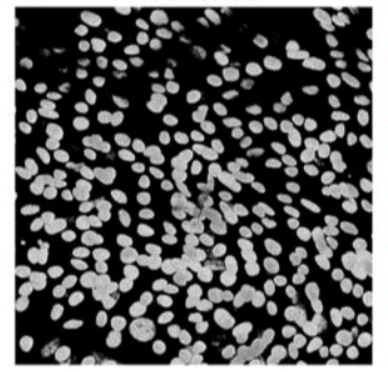

merged

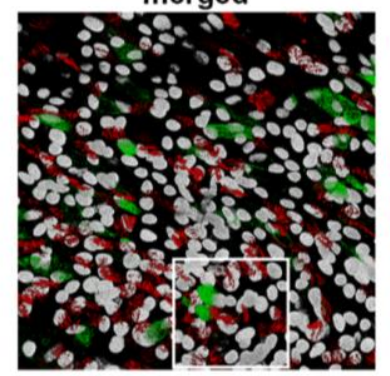

B
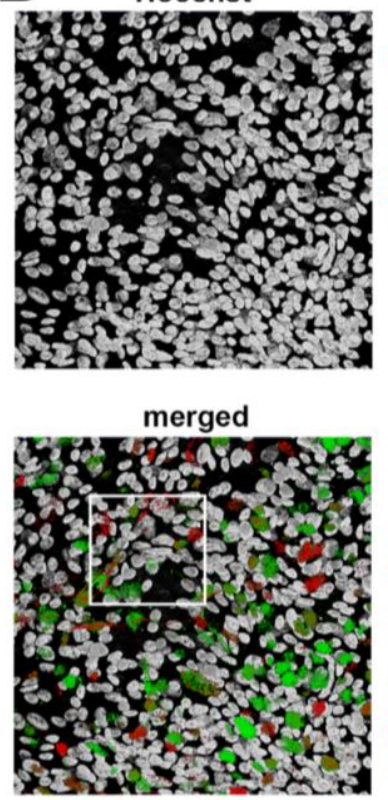

Tubulin $\beta 4$
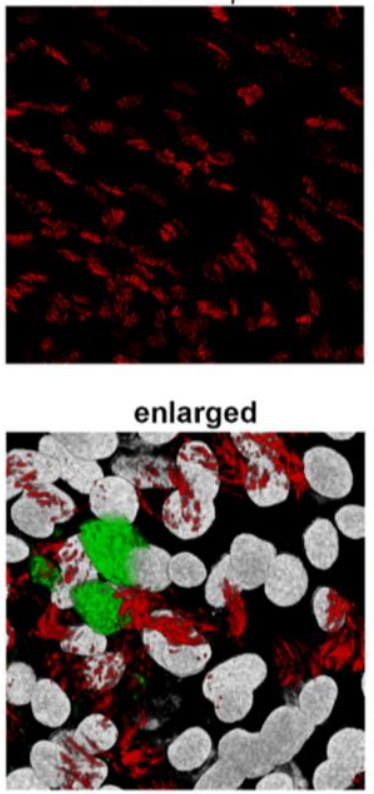

Mucin $5 \mathrm{~A} / \mathrm{C}$

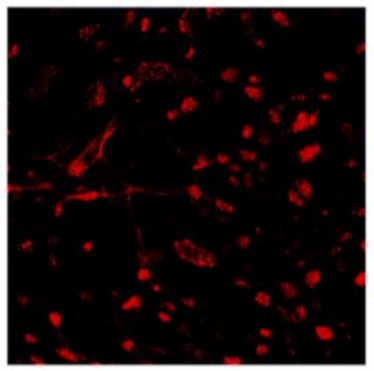

enlarged

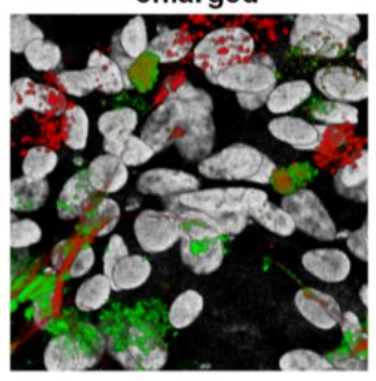

Keratin 5

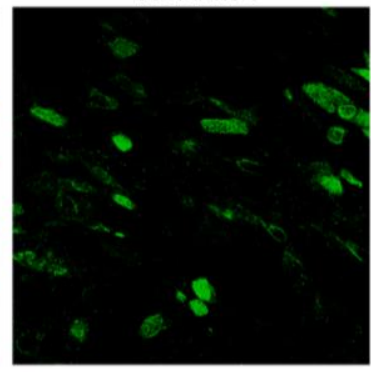

side view

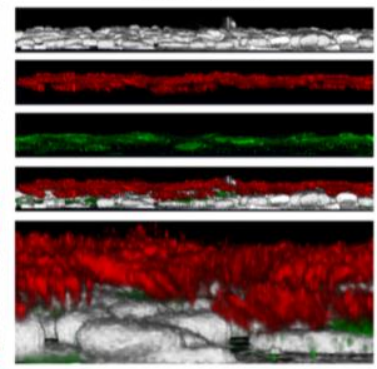

Uteroglobin (CC10)

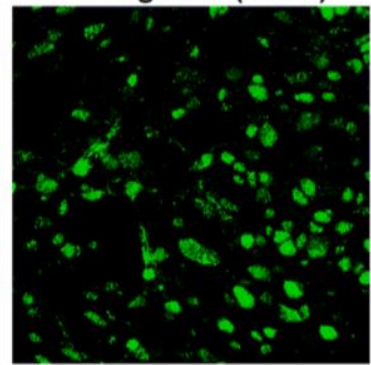

side view

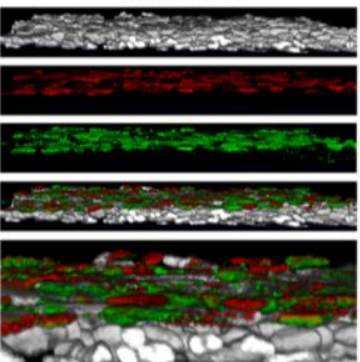

Figure 8.2: Differentiated HBE cells. (A) Top view and side view of BMS-derived patient ALI cell cultures stained with Tubulin- $\beta 4$ (red, ciliated cells) and Keratin 5 (green, basal cells). (B) Top view and side view of staining with Mucin 5B (red, Goblet cells) and CC10 (green, Club cells) of BMS derived patient ALI cell cultures. 
Previous studies have indicated that epithelial cells from IPF patients have an increased expression of the NADPH oxidase NOX4 that enhances epithelial oxidant production (37) whereas cellular antioxidant systems are decreased (8-13), thereby causing a redox imbalance. Therefore, we assessed NOX4 expression as well as the expression of various antioxidants in HBE cells from both controls and IPF patients. NOX4 was upregulated in epithelial cells derived from patients with IPF in comparison to control cells ( $3.98 \pm 3.22$ vs $1.26 \pm 1.07$ ) (Figure $8.3 \mathrm{~A})$, although not significantly $(p=0.23)$ and not correlated with disease severity as indicated by the diffusing capacity for carbon monoxide (DLCO) (Figure 8.3B). Interestingly, NOX4 expression differs notable between individual IPF patients. Gene expression of antioxidant genes was not significantly different between IPF patients and controls, although the oxidant-sensitive transcription factor NRF2 as well as CAT and SOD2 were slightly downregulated in IPF patients whereas HO-1 and SOD1 were slightly upregulated (Figure 8.3).

A

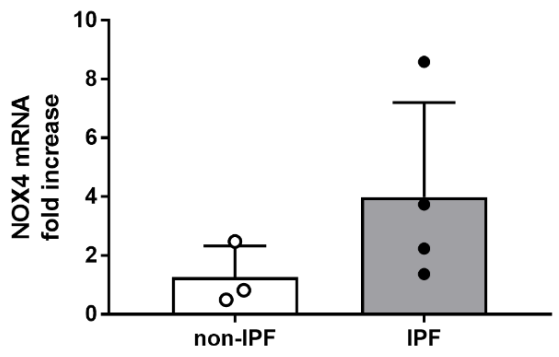

C
B

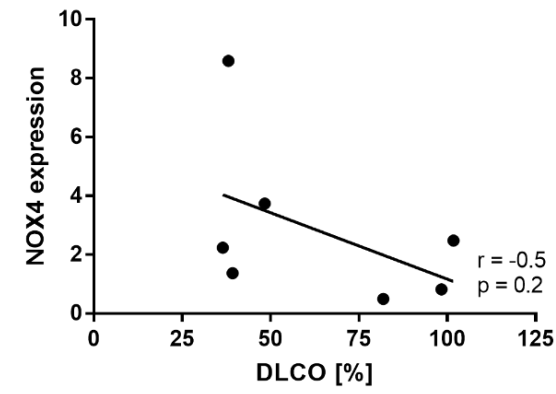

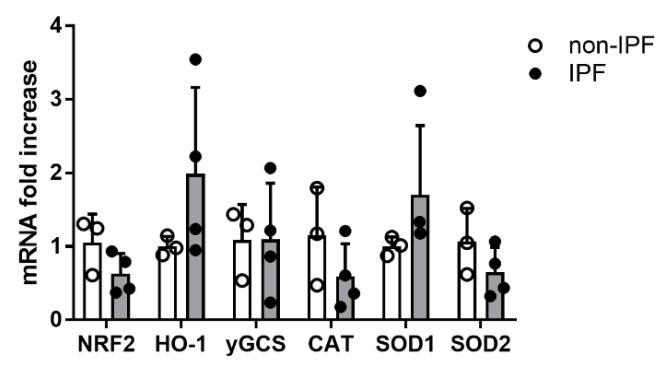

Figure 8.3: $\mathrm{HBE}$ cells derived from IPF patients have different gene expression levels of NOX4 and endogenous antioxidants. (A) NOX4 expression levels in HBE cells from IPF patients $(n=4)$ and controls $(n=3)$. Data are presented as mean \pm SD. (B) Correlation of NOX4 expression with DLCO (\%). (C) Antioxidant gene expression in HBE cells from IPF patients $(n=4)$ and controls $(n=3)$. Data are presented as mean \pm SD.

To assess the effects of the drugs, cells were treated for 24 hours with a range of doses similar to those previously applied $(22,23,38)$ in published studies. Notably, others have used pirfenidone in concentrations up to $10 \mathrm{mM}$ (39), however, to adhere to physiologically relevant concentrations we stimulated cells with maximal $1 \mathrm{mM}$. Initially, 
the effects of different dose ranges of pirfenidone $(1 \mathrm{mM}-100 \mu \mathrm{M})$, nintedanib $(1 \mu \mathrm{M}$ $0.01 \mu \mathrm{M})$ and saracatinib $(10 \mu \mathrm{M}-0.1 \mu \mathrm{M})$ on cell viability were analyzed using MTT cytotoxicity assay to determine mitochondrial metabolic activity (Figure 8.4A). Since the highest concentration of both nintedanib and saracatinib reduced the cell viability below $90 \%$ (Figure 8.4A), these doses were excluded. Next, cells were treated with the highest non-cytotoxic concentrations of the drugs (i.e. $1 \mathrm{mM}$ pirfenidone, $0.1 \mu \mathrm{M}$ nintedanib and $1 \mu \mathrm{M}$ saracatinib) and $\mathrm{LDH}$ release, an indication of cell damage, was measured. No significant LDH release was determined after stimulation for 24 hours (Figure 8.4B), indicating that the concentrations applied were well tolerated for the chosen treatment period.

A

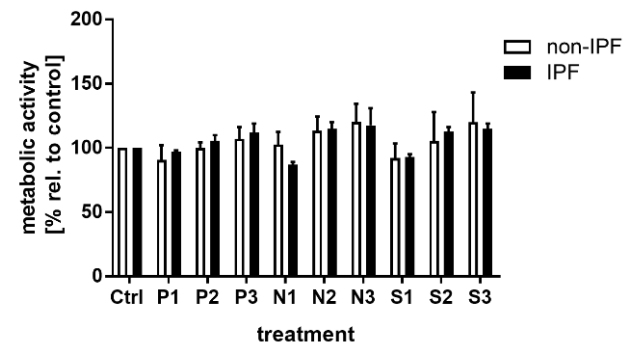

B

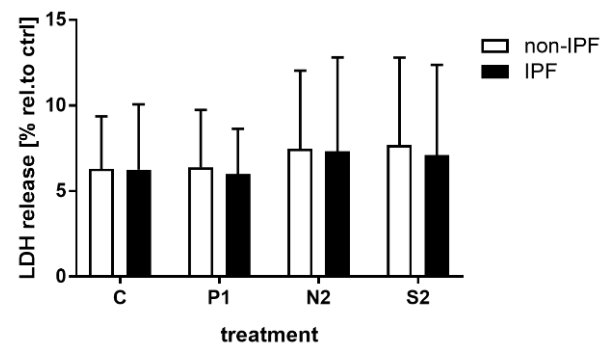

Figure 8.4: Pirfenidone, nintedanib and saracatinib do not influence cell viability. (A) MTT assay and (B) LDH assay after treatment with different doses of pirfenidone (P1: $1 \mathrm{mM}, \mathrm{P} 2: 500 \mu \mathrm{M}, \mathrm{P} 3: 100 \mu \mathrm{M})$, nintedanib (N1:1 $\mu \mathrm{M}, \mathrm{N2}: 0.1 \mu \mathrm{M}, \mathrm{N} 3: 0.01 \mu \mathrm{M})$ and saracatinib (S1: $10 \mu \mathrm{M}, \mathrm{S2}: 1 \mu \mathrm{M}, \mathrm{S3}: 0.1 \mu \mathrm{M})$ for 24 hrs in IPF patients $(\mathrm{n}=4)$ and controls $(n=3)$. Data are presented as mean \pm SD.

Next, we examined the effect of the selected concentrations pirfenidone, nintedanib and saracatinib on NOX4 and antioxidant gene expression. nintedanib reduced NOX4 expression in IPF patients $(0.68 \pm 0.16)$, although this effect was not statistically significant $(p=0.07)$ (Figure 8.5A). NRF2, HO-1, $y G C S$, SOD1, SOD2 and CAT expression did not change after treatment with pirfenidone, nintedanib or saracatinib (Figure 8.5B-G) Notably, in two patients who had higher expression of NOX4 (2.73 and 1.19 respectively) and lower expression of NRF2 ( 0.73 and 1.34 respectively), pirfenidone was more effective and did induce the gene expression of various antioxidant genes (see supplemental data E8.1). 
A

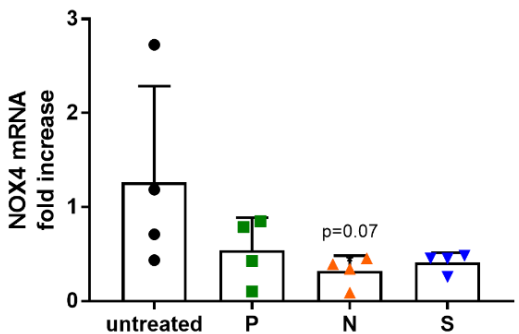

C

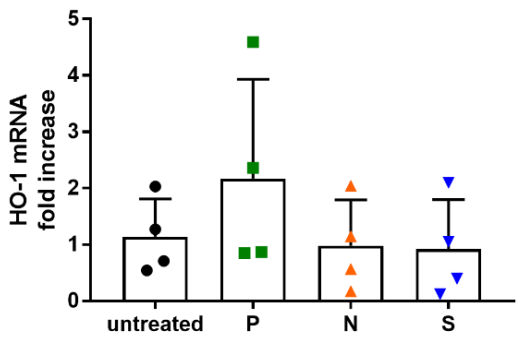

$\mathbf{E}$

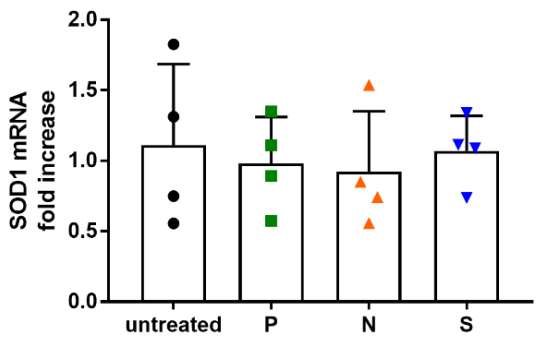

G

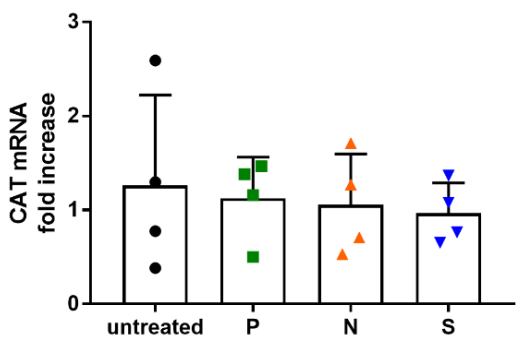

B

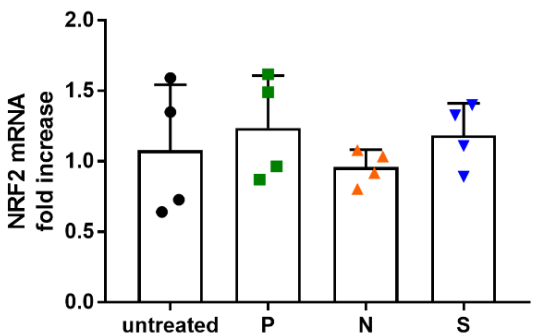

D

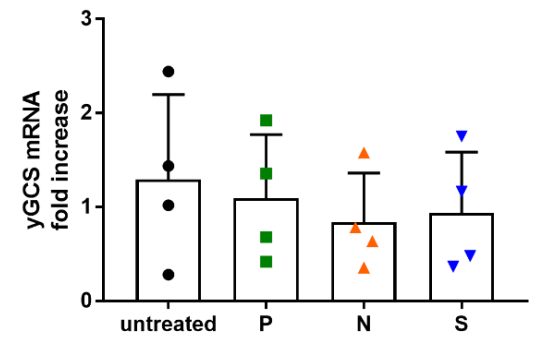

$\mathbf{F}$

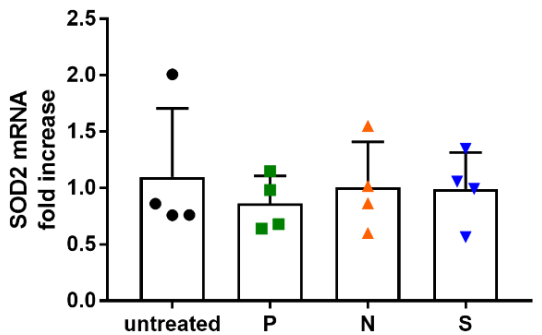

Figure 8.5: NOX4 and antioxidant gene expression in HBE cells from IPF patients are not significantly affected by stimulation with pirfenidone (P), nintedanib (N) and saracatinib (S) for 24 hours. (A) NOX4 expression, (B) NRF2 expression, (C) HO-1 expression, (D) $\mathrm{YGCS}$ expression, (E) CAT expression, (F) SOD1 and (G) SOD2 expression in HBE cells from IPF patients $(n=4)$ after stimulation with pirfenidone $(1 \mathrm{mM})$, nintedanib $(0.1 \mu \mathrm{M})$ and saracatinib $(1 \mu \mathrm{M})$ for $24 \mathrm{hrs}$. The change in gene expression was calculated for every individual IPF patient. Data are presented as mean \pm SD. 
IPF patients often suffer from chronic inflammation and therefore the secretion of interleukin (IL)- 6 and IL-8 was determined using ELISA. Intriguingly, most HBE cells isolated from IPF patients already secreted increased levels of IL-6 and IL-8 compared to HBE cells from non-IPF patients, $197.2 \mathrm{pg} / \mathrm{mL} \pm 145.3 \mathrm{pg} / \mathrm{mL}$ vs $20.62 \mathrm{pg} / \mathrm{mL} \pm 23.62 \mathrm{pg} / \mathrm{mL}$ ( $\mathrm{p}=$ 0.09 ) and $1321 \mathrm{pg} / \mathrm{mL} \pm 355.3 \mathrm{pg} / \mathrm{mL}$ vs $396.7 \mathrm{pg} / \mathrm{mL} \pm 278.8 \mathrm{pg} / \mathrm{mL}$ ( $\mathrm{p}=0.014)$ respectively (Figure $8.6 \mathrm{~A}$ and $\mathrm{B}$ ). Twenty four hours of stimulation with pirfenidone, nintedanib and saracatinib had various effects on HBE cells of IPF patients as IL-6 production was mostly unaffected (Figure $8.6 \mathrm{C}$ ), whereas IL-8 release was significantly reduced $(p=0.02)$ after treatment with saracatinib but unaltered by the two anti-fibrotic drugs (Figure 8.6D).

A

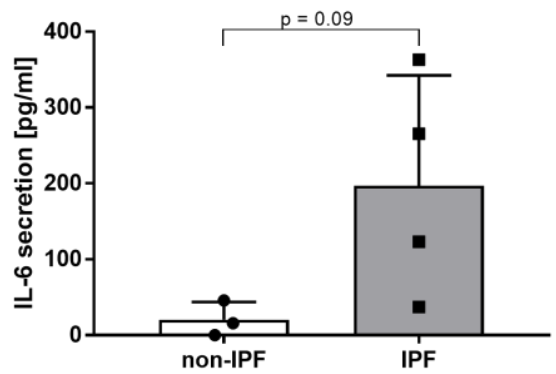

C

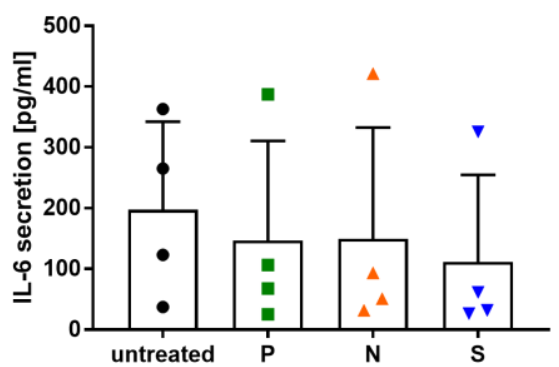

B

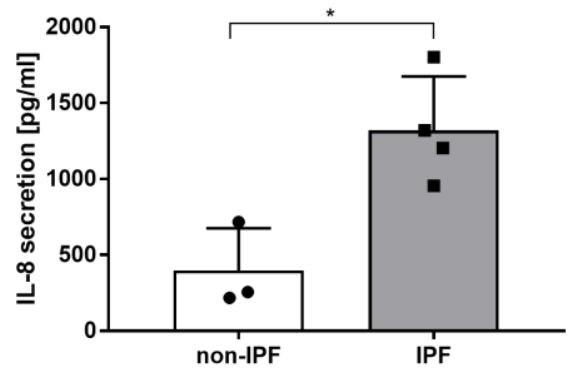

D

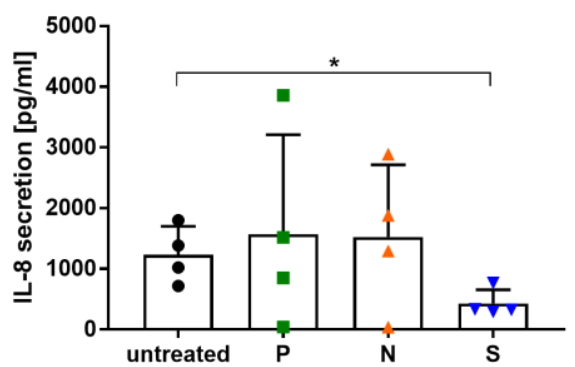

Figure 8.6: IL-6 (A) and IL-8 (B) secretion by HBE cells from IPF patients is increased and IL-8 can be reduced after incubation with saracatinib for 24 hours. (A) IL-6 and (B) IL-8 secretion in HBE cells from IPF patients $(n=4)$ and controls $(n=3)$. (C) IL-6 and (D) IL-8 secretion in HBE cells from IPF patients after stimulation with pirfenidone (1 $m M)$, nintedanib $(0.1 \mu M)$ or saracatinib $(1 \mu M)$ for 24 hrs $(n=4)$. Data are presented as mean \pm SD. $\left({ }^{*} P<0.05\right)$. 


\section{Discussion}

IPF is a progressive chronic interstitial lung disease, which leads to the remodeling of the lung architecture causing loss of lung function, subsequent respiratory failure and ultimately death. Repetitive injury to the lung epithelium and dysregulated repair represent important features of the development of IPF as they lead to increased apoptosis and abnormal regeneration of the lung epithelium (40). Recently, two drugs have been approved for IPF treatment, pirfenidone and nintedanib, which have shown to slow down the progression of the disease $(41,42)$. Moreover, SFK inhibition has been suggested as alternative treatment strategy for IPF (27) but its effect on IPF pathology remains to be elucidated.

In this pilot study, we demonstrated that cells from the ELF can be differentiated into a complete bronchial epithelium, consisting of ciliated epithelial, basal, goblet and club cells. Our preliminary results indicate that responses of important redox readouts to pirfenidone and nintedanib largely differ between individual IPF patients. Additionally, our data suggest that nintedanib as well as saracatinib can reduce NOX4 expression, although not significantly. Saracatinib also reduces inflammatory cytokine release in HBE cells from 3 of the 4 included IPF patients. This pilot study was designed to setup methodology for a larger scale study, ultimately designed to identify predictors for treatment efficacy, thereby supporting the prediction of treatment outcome in IPF patients in the future.

IPF is a complex heterogeneous disease and although there are two new drugs available, they are not fully effective in every patient. Recently, it has been suggested that a shift towards a personalized treatment approach in IPF could be a realistic goal to improve treatment of the affected patients (43-45). However, to make use of personalized medicine, subgroups of IPF patients have to be identified first to develop targeted therapies and maximize the outcome of treatment strategies. A possible trait for defining such subgroups is the redox status as oxidative stress has been implicated in the pathogenesis of IPF and various studies have demonstrated increased markers of oxidative damage in patients suffering from this disease (46-48). Moreover, antioxidants and detoxification enzymes show a diminished expression in patients with IPF (8-13). However, not all patients might have an increased oxidant burden, as can also be deducted from the spread in NOX4 and AOX expression observed in the present study, which might explain why antioxidant therapy has failed to be generally effective in IPF thus far. Instead, patients with increased NOX4 expression might benefit from antioxidant treatment whereas patients without an altered redox balance might rather benefit from a different treatment approach with for example one of the two antifibrotic drugs. An alternative way of phenotyping IPF patients could be by inflammatory status as this study has once more showed that some but not all patients present significantly elevated levels of pro-inflammatory cytokines. Anti-inflammatory treatment strategies failed and it is still 
not clear what function inflammation plays in the progression of the disease (49), but it can be anticipated that immunosuppressants may be helpful in a special subgroup of IPF patients with increased pulmonary proinflammatory cytokine production.

Our study is the first study that uses pulmonary bronchial epithelial cells obtained from yet untreated IPF patients to evaluate treatment effects on markers of antioxidant genes and pro-inflammatory cytokine release. One limitation associated with this study is the use of bronchial epithelial cells as IPF primarily impacts the alveolar epithelium (50). However, it has recently been suggested that injury to the bronchial epithelium also promotes the development of pulmonary fibrosis (51).

Our study has some limitations: as this was only a pilot study, the sample size was rather limited and needs to be enlarged in the future. Additionally, the working mechanisms of pirfenidone and nintedanib in epithelial cells remain to be elucidated. Combined with the fact that nintedanib also inhibits non-receptor tyrosine kinases including SFK (52) suggests that part of the working mechanism of this antifibrotic drug is indeed via SFK inhibition.

Since saracatinib is not yet approved for fibrotic diseases, its mechanism of action still needs to be investigated as well. We did not observe an effect of the drugs in every patient, which could be associated with adjusting concentrations and time points of stimulation. Most performed studies have used concentrations of nintedanib and pirfenidone which would not be achievable in humans as the concentrations found in the plasma of patients are approximately $0.1 \mu \mathrm{M}$ for nintedanib (53) and $100 \mu \mathrm{M}$ (54) for pirfenidone. We used the same concentration for nintedanib but chose a higher concentration ( $1 \mathrm{mM}$ ) for pirfenidone as this has commonly been used in in vitro studies thus far and did not induce cell death in the HBE cells applied in the present study $(38,55)$. However, the variable responses to the drugs could also indicate that individual patients have different responses to different drugs and further strengthen the call for patient stratifying, followed by tailored treatment based on specific disease progression characteristics.

In conclusion, there are still many open questions regarding the best treatment strategies for IPF patients. This pilot study suggests that responsiveness to treatment varies significantly between individual IPF patients, presumably due to a differently affected redox status, and that treatment should be personalized to maximize its benefit, thereby decreasing the burden of disease and mortality associated with the disease. 


\section{Acknowledgments}

CV greatly appreciates the research support from the Lung Foundation Netherlands (Project number: 9.2.17.214FE). 


\section{References}

1. Raghu G, Chen SY, Yeh WS, Maroni B, Li Q, Lee YC, et al. Idiopathic pulmonary fibrosis in US Medicare beneficiaries aged 65 years and older: incidence, prevalence, and survival, 2001-11. Lancet Respir Med. 2014;2(7):566-72.

2. Nalysnyk L, Cid-Ruzafa J, Rotella P, Esser D. Incidence and prevalence of idiopathic pulmonary fibrosis: review of the literature. Eur Respir Rev. 2012;21(126):355-61.

3. Navaratnam V, Fleming KM, West J, Smith CJ, Jenkins RG, Fogarty A, et al. The rising incidence of idiopathic pulmonary fibrosis in the U.K. Thorax. 2011;66(6):462-7.

4. Raghu G, Weycker D, Edelsberg J, Bradford WZ, Oster G. Incidence and prevalence of idiopathic pulmonary fibrosis. Am J Respir Crit Care Med. 2006;174(7):810-6.

5. Mora AL, Rojas M, Pardo A, Selman M. Emerging therapies for idiopathic pulmonary fibrosis, a progressive age-related disease. Nature reviews Drug discovery. 2017;16(11):810.

6. du Bois RM. Strategies for treating idiopathic pulmonary fibrosis. Nature reviews Drug discovery. 2010;9(2):129-40.

$7 . \quad H e c k e r \mathrm{~L}$, Cheng J, Thannickal VJ. Targeting NOX enzymes in pulmonary fibrosis. Cell Mol Life Sci. 2012;69(14):2365-71.

8. Veith C, Drent M, Bast A, van Schooten FJ, Boots AW. The disturbed redox-balance in pulmonary fibrosis is modulated by the plant flavonoid quercetin. Toxicol Appl Pharmacol. 2017;336:40-8.

9. Cantin AM, Hubbard RC, Crystal RG. Glutathione deficiency in the epithelial lining fluid of the lower respiratory tract in idiopathic pulmonary fibrosis. Am Rev Respir Dis. 1989;139(2):370-2.

10. Odajima N, Betsuyaku T, Nagai K, Moriyama C, Wang DH, Takigawa T, et al. The role of catalase in pulmonary fibrosis. Respir Res. 2010;11:183.

11. Kinnula VL, Hodgson UA, Lakari EK, Tan RJ, Sormunen RT, Soini YM, et al. Extracellular superoxide dismutase has a highly specific localization in idiopathic pulmonary fibrosis/usual interstitial pneumonia. Histopathology. 2006;49(1):66-74.

12. Anathy V, Lahue KG, Chapman DG, Chia SB, Casey DT, Aboushousha R, et al. Reducing protein oxidation reverses lung fibrosis. Nature medicine. 2018.

13. Vuorinen K, Ohlmeier S, Lepparanta O, Salmenkivi K, Myllarniemi M, Kinnula VL. Peroxiredoxin II expression and its association with oxidative stress and cell proliferation in human idiopathic pulmonary fibrosis. J Histochem Cytochem. 2008;56(10):951-9.

14. Hecker L, Vittal R, Jones T, Jagirdar R, Luckhardt TR, Horowitz JC, et al. NADPH oxidase-4 mediates myofibroblast activation and fibrogenic responses to lung injury. Nat Med. 2009;15(9):1077-81.

15. Jiang F, Liu GS, Dusting GJ, Chan EC. NADPH oxidase-dependent redox signaling in TGFbeta-mediated fibrotic responses. Redox Biol. 2014;2:267-72.

16. Kolb M, Bonella F, Wollin L. Therapeutic targets in idiopathic pulmonary fibrosis. Respiratory medicine. 2017;131:49-57.

17. Kwapiszewska G, Gungl A, Wilhelm J, Marsh LM, Thekkekara Puthenparampil H, Sinn K, et al. Transcriptome profiling reveals the complexity of pirfenidone effects in idiopathic pulmonary fibrosis. Eur Respir J. 2018;52(5).

18. Misra HP, Rabideau C. Pirfenidone inhibits NADPH-dependent microsomal lipid peroxidation and scavenges hydroxyl radicals. Mol Cell Biochem. 2000;204(1-2):119-26.

19. Giri SN, Leonard S, Shi X, Margolin SB, Vallyathan V. Effects of pirfenidone on the generation of reactive oxygen species in vitro. J Environ Pathol Toxicol Oncol. 1999;18(3):169-77. 20. Oku H, Shimizu T, Kawabata T, Nagira M, Hikita I, Ueyama A, et al. Antifibrotic action of pirfenidone and prednisolone: different effects on pulmonary cytokines and growth factors in bleomycin-induced murine pulmonary fibrosis. Eur J Pharmacol. 2008;590(1-3):400-8. 
21. Conte $\mathrm{E}$, Gili E, Fagone $\mathrm{E}$, Fruciano $\mathrm{M}$, lemmolo $\mathrm{M}$, Vancheri $\mathrm{C}$. Effect of pirfenidone on proliferation, TGF-beta-induced myofibroblast differentiation and fibrogenic activity of primary human lung fibroblasts. Eur J Pharm Sci. 2014;58:13-9.

22. Hostettler KE, Zhong J, Papakonstantinou E, Karakiulakis G, Tamm M, Seidel P, et al. Antifibrotic effects of nintedanib in lung fibroblasts derived from patients with idiopathic pulmonary fibrosis. Respir Res. 2014;15:157.

23. Rangarajan S, Kurundkar A, Kurundkar D, Bernard K, Sanders YY, Ding Q, et al. Novel Mechanisms for the Antifibrotic Action of Nintedanib. Am J Respir Cell Mol Biol. 2016;54(1):51-9.

24. Hostettler KE, Zhong J, Papakonstantinou E, Karakiulakis G, Tamm M, Seidel P, et al. Antifibrotic effects of nintedanib in lung fibroblasts derived from patients with idiopathic pulmonary fibrosis. Respir Res. 2014;15:157.

25. Sato S, Shinohara S, Hayashi S, Morizumi S, Abe S, Okazaki H, et al. Anti-fibrotic efficacy of nintedanib in pulmonary fibrosis via the inhibition of fibrocyte activity. Respir Res. 2017;18(1):172.

26. Lehmann M, Buhl L, Alsafadi HN, Klee S, Hermann S, Mutze K, et al. Differential effects of Nintedanib and Pirfenidone on lung alveolar epithelial cell function in ex vivo murine and human lung tissue cultures of pulmonary fibrosis. Respir Res. 2018;19(1):175.

27. Hu M, Che P, Han X, Cai GQ, Liu G, Antony V, et al. Therapeutic targeting of SRC kinase in myofibroblast differentiation and pulmonary fibrosis. J Pharmacol Exp Ther. 2014;351(1):87-95.

28. Roskoski R, Jr. Src protein-tyrosine kinase structure, mechanism, and small molecule inhibitors. Pharmacol Res. 2015;94:9-25.

29. Thomas SM, Brugge JS. Cellular functions regulated by Src family kinases. Annu Rev Cell Dev Biol. 1997;13:513-609.

30. Grimminger F, Gunther A, Vancheri C. The role of tyrosine kinases in the pathogenesis of idiopathic pulmonary fibrosis. Eur Respir J. 2015;45(5):1426-33.

31. Lu YY, Zhao XK, Yu L, Qi F, Zhai B, Gao CQ, et al. Interaction of Src and Alpha-V Integrin Regulates Fibroblast Migration and Modulates Lung Fibrosis in A Preclinical Model of Lung Fibrosis. Sci Rep. 2017;7:46357.

32. Selman M, King TE, Pardo A, American Thoracic S, European Respiratory S, American College of Chest $P$. Idiopathic pulmonary fibrosis: prevailing and evolving hypotheses about its pathogenesis and implications for therapy. Ann Intern Med. 2001;134(2):136-51.

33. Warsinske HC, Wheaton AK, Kim KK, Linderman JJ, Moore BB, Kirschner DE. Computational Modeling Predicts Simultaneous Targeting of Fibroblasts and Epithelial Cells Is Necessary for Treatment of Pulmonary Fibrosis. Front Pharmacol. 2016;7:183.

34. Kahn N, Kuner R, Eberhardt R, Meister M, Muley T, Winteroll S, et al. Gene expression analysis of endobronchial epithelial lining fluid in the evaluation of indeterminate pulmonary nodules. J Thorac Cardiovasc Surg. 2009;138(2):474-9.

35. Raghu G, Collard HR, Egan JJ, Martinez FJ, Behr J, Brown KK, et al. An official ATS/ERS/JRS/ALAT statement: idiopathic pulmonary fibrosis: evidence-based guidelines for diagnosis and management. Am J Respir Crit Care Med. 2011;183(6):788-824.

36. Wu R. Growth of human lung tumor cells in culture. Culture of Human Tumor Cells Pfragner R, Freshney RI (eds) Wiley-Liss Inc: Hoboken, NJ. 2004:1-21.

37. Carnesecchi S, Deffert C, Donati Y, Basset O, Hinz B, Preynat-Seauve O, et al. A key role for NOX4 in epithelial cell death during development of lung fibrosis. Antioxidants \& redox signaling. 2011;15(3):607-19.

38. Knuppel L, Ishikawa Y, Aichler M, Heinzelmann K, Hatz R, Behr J, et al. A Novel Antifibrotic Mechanism of Nintedanib and Pirfenidone. Inhibition of Collagen Fibril Assembly. Am J Respir Cell Mol Biol. 2017;57(1):77-90.

39. Nakayama S, Mukae H, Sakamoto N, Kakugawa T, Yoshioka S, Soda H, et al. Pirfenidone inhibits the expression of HSP47 in TGF-beta1-stimulated human lung fibroblasts. Life Sci. 2008;82(34):210-7. 
40. Camelo A, Dunmore R, Sleeman MA, Clarke DL. The epithelium in idiopathic pulmonary fibrosis: breaking the barrier. Front Pharmacol. 2014;4:173.

41. King TE, Jr., Bradford WZ, Castro-Bernardini S, Fagan EA, Glaspole I, Glassberg MK, et al. A phase 3 trial of pirfenidone in patients with idiopathic pulmonary fibrosis. $\mathrm{N}$ Engl J Med. 2014;370(22):2083-92.

42. Richeldi L, du Bois RM, Raghu G, Azuma A, Brown KK, Costabel U, et al. Efficacy and safety of nintedanib in idiopathic pulmonary fibrosis. N Engl J Med. 2014;370(22):2071-82.

43. Spagnolo $P$, Oldham JM, Jones MG, Lee JS. Personalized medicine in interstitial lung diseases. Curr Opin Pulm Med. 2017;23(3):231-6.

44. Sato S, Kolb MRJ. Personalised medicine for IPF: getting closer, but not there yet. Lancet Respir Med. 2017;5(11):836-7.

45. Thannickal VJ, Antony VB. Is personalized medicine a realistic goal in idiopathic pulmonary fibrosis? Expert Rev Respir Med. 2018;12(6):441-3.

46. Bargagli E, Olivieri C, Bennett D, Prasse A, Muller-Quernheim J, Rottoli P. Oxidative stress in the pathogenesis of diffuse lung diseases: a review. Respir Med. 2009;103(9):1245-56.

47. Psathakis K, Mermigkis D, Papatheodorou G, Loukides S, Panagou P, Polychronopoulos V, et al. Exhaled markers of oxidative stress in idiopathic pulmonary fibrosis. Eur J Clin Invest. 2006;36(5):362-7.

48. Lenz AG, Costabel U, Maier KL. Oxidized BAL fluid proteins in patients with interstitial lung diseases. The European respiratory journal. 1996;9(2):307-12.

49. Bringardner BD, Baran CP, Eubank TD, Marsh CB. The role of inflammation in the pathogenesis of idiopathic pulmonary fibrosis. Antioxidants \& redox signaling. 2008;10(2):287-301.

50. Martinez FJ, Collard HR, Pardo A, Raghu G, Richeldi L, Selman M, et al. Idiopathic pulmonary fibrosis. Nat Rev Dis Primers. 2017;3:17074.

51. Evans CM, Fingerlin TE, Schwarz MI, Lynch D, Kurche J, Warg L, et al. Idiopathic Pulmonary Fibrosis: A Genetic Disease That Involves Mucociliary Dysfunction of the Peripheral Airways. Physiol Rev. 2016;96(4):1567-91.

52. Wollin L, Wex E, Pautsch A, Schnapp G, Hostettler KE, Stowasser S, et al. Mode of action of nintedanib in the treatment of idiopathic pulmonary fibrosis. Eur Respir J. 2015;45(5):1434-45.

53. Schmid U, Liesenfeld KH, Fleury A, Dallinger C, Freiwald M. Population pharmacokinetics of nintedanib, an inhibitor of tyrosine kinases, in patients with non-small cell lung cancer or idiopathic pulmonary fibrosis. Cancer Chemother Pharmacol. 2018;81(1):89-101.

54. Shi S, Wu J, Chen H, Chen H, Wu J, Zeng F. Single- and multiple-dose pharmacokinetics of pirfenidone, an antifibrotic agent, in healthy Chinese volunteers. J Clin Pharmacol. 2007;47(10):1268-76.

55. Lehtonen ST, Veijola A, Karvonen H, Lappi-Blanco E, Sormunen R, Korpela S, et al. Pirfenidone and nintedanib modulate properties of fibroblasts and myofibroblasts in idiopathic pulmonary fibrosis. Respir Res. 2016;17:14. 


\section{Supplemental data}

Table E8.1: Gene expression of NOX4, NRF2, HO-1, үGCS, SOD1, SOD2 and CAT for individual IPF patients $(P)$ without and with treatment with pirfenidone $(1 \mathrm{mM})$, nintedanib $(0.1 \mu \mathrm{M})$ and saracatinib $(1 \mu \mathrm{M})$ for $24 \mathrm{hrs}$.

\begin{tabular}{|c|c|c|c|c|c|c|c|}
\hline & NOX4 & NRF2 & HO-1 & yGCS & SOD1 & SOD2 & CAT \\
\hline P1 & 2.73 & 0.73 & 0.55 & 0.28 & 0.75 & 2.01 & 0.78 \\
\hline $\mathbf{P}$ & 0.79 & 1.49 & 2.36 & 1.36 & 1.11 & 0.98 & 1.47 \\
\hline $\mathbf{N}$ & 0.46 & 0.92 & 1.15 & 0.78 & 0.74 & 0.86 & 0.71 \\
\hline $\mathbf{S}$ & 0.48 & 1.40 & 2.10 & 1.75 & 1.34 & 1.35 & 1.37 \\
\hline P2 & 0.71 & 1.59 & 1.27 & 2.44 & 1.83 & 0.76 & 2.59 \\
\hline $\mathbf{P}$ & 0.85 & 0.87 & 0.85 & 0.42 & 0.57 & 0.68 & 0.50 \\
\hline $\mathbf{N}$ & 0.39 & 0.80 & 0.57 & 0.36 & 0.56 & 1.02 & 0.53 \\
\hline $\mathbf{S}$ & 0.26 & 0.89 & 0.12 & 0.37 & 0.74 & 1.06 & 0.65 \\
\hline P3 & 1.19 & 1.35 & 0.71 & 1.02 & 1.31 & 0.86 & 1.30 \\
\hline $\mathbf{P}$ & 0.10 & 1.62 & 4.59 & 1.92 & 1.35 & 0.64 & 1.38 \\
\hline $\mathbf{N}$ & 0.09 & 1.03 & 2.04 & 1.58 & 1.54 & 0.60 & 1.71 \\
\hline$S$ & 0.46 & 1.11 & 1.05 & 1.16 & 1.11 & 0.57 & 0.76 \\
\hline P4 & 0.44 & 0.64 & 2.03 & 1.44 & 0.56 & 0.76 & 0.38 \\
\hline $\mathbf{P}$ & 0.43 & 0.97 & 0.87 & 0.68 & 0.89 & 1.15 & 1.16 \\
\hline $\mathbf{N}$ & 0.34 & 1.10 & 0.17 & 0.64 & 0.85 & 1.55 & 1.27 \\
\hline$S$ & 0.45 & 1.33 & 0.40 & 0.48 & 1.09 & 0.99 & 1.08 \\
\hline
\end{tabular}






\section{Chapter 9}

Summary and Discussion 


\section{Main findings: Just scavenging oxidants is not the solution in IPF}

Idiopathic pulmonary fibrosis (IPF) is characterized by the presence of oxidative stress and IPF patients demonstrate increased markers of oxidative damage $(1,2)$. Despite this, antioxidant therapies have not been proven effective in treating IPF and the role of oxidative stress is still not completely clear. The aim of this thesis was to explore the importance of oxidant regulation in pulmonary fibrosis. To this end, 1) the role of reactive oxygen species (ROS) was studied by scavenging ROS with the dietary antioxidant quercetin, 2) the role of oxidants specifically produced by the NADPH oxidase NOX4 in the activation of SRC family kinases was investigated and 3) the effect of SRC family kinase (SFK) inhibition was evaluated to mitigate specific features of IPF.

In Chapter $\mathbf{3}$ we examined the potentially protective effects of the antioxidant quercetin on oxidant production and pro-inflammatory cytokine secretion in human bronchial epithelial cells as well as ex vivo in blood of IPF patients. Quercetin reduced bleomycininduced ROS production, while also boosting Nrf2 activity, and the release of inflammatory cytokines in vitro and ex vivo in blood of IPF patients. One-week of dietary quercetin supplementation in mice increased the quercetin concentration in the plasma, however, not in the lungs, and slightly enhanced the pulmonary expression of Nrf2responsive genes (chapter 4). Upon bleomycin exposure, $\mathrm{Nrf2}^{+/+}$quercetin-fed mice displayed reduced expression of collagen and fibronectin and as well as reduced pulmonary inflammatory lesions. Furthermore, quercetin reduced pulmonary gene expression of TNF $\alpha$ and $\mathrm{KC}$, but not their plasma levels, and diminished malondialdehydedG DNA adducts. In Nrf2\% animals, no effect of the dietary antioxidant on either histology or inflammatory lesions was observed upon exposure to bleomycin suggesting that quercetin protects against bleomycin-induced pulmonary damage via the induction of Nrf2. However, quercetin supplementation could not reverse the bleomycin-induced damage indicating that quercetin is not sufficient to abrogate the development of pulmonary fibrosis.

One major pulmonary source of oxidants is the NADPH oxidase (NOX) 4, however, targets of NOX4-dependent ROS are largely unknown. We identified in chapter 5 that NOX4 oxidized the SFK member FYN thereby activating it in response to TGF- $\beta 1$ in human bronchial epithelial cells. When FYN was inhibited, it reduced mitochondrial ROS production leading to reduction of DNA damage and expression of fibronectin. Inhibition of SFK by saracatinib reduced fibroblast activation, ROS production as well as inflammatory cytokine release, as presented in chapter 6. Intriguingly, in fibroblasts TGF$\beta 1$ activated the SFK member YES which contributed to the expression of extracellular matrix genes in fibroblasts. Furthermore, the TGF-induced increase in NOX4 expression 
was diminished in YES-silenced cells indicating that the SFK member YES promoted fibroblast activation potentially through the induction of NOX4.

In chapter 7, we demonstrated that bronchial epithelial cells from IPF patients depict an increased activation of SFK, and inhibition of SFK by AZD reduced markers of mitochondrial dysfunction by increasing the expression of genes associated with mitochondrial biogenesis and mitophagy. Additionally, SFK inhibition reduced LDH release, indicating cell damage and expression of the pro-apoptotic gene BAX as well as NOX4. In differentiated bronchial epithelial cells, NOX4 expression is slightly increased in IPF patients compared to control epithelial cells but differs on individual level (chapter 8). Intriguingly, in patients with higher NOX4 expression, pirfenidone induced antioxidant genes. Furthermore, IL-8 was significantly increased in HBE cells derived from IPF patients and treatment with saracatinib reduced this elevated IL- 8 secretion significantly. After comparing the effects of the FDA-approved drugs pirfenidone and nintedanib to the SFK inhibitor saracatinib in differentiated bronchial epithelial cells, we propose that IPF patients might benefit from personalized treatment as responsiveness to the different drugs varied between individual patients and was dependent on their redox and inflammatory status. 


\section{Discussion: targeting oxidative stress or redox signaling?}

\section{Reactive oxygen species}

The oxidative stress theory

ROS are involved in the regulation of cellular homeostasis by controlling cell proliferation as well as differentiation. Additionally, ROS have various physiological functions in host defense and immune regulation. They are produced as by-products of cellular metabolism during oxidative phosphorylation in the electron transport chain in the mitochondria or by several enzyme systems such as the NADPH oxidases (NOX) (3). Furthermore, the lungs are also exposed to exogenous sources of ROS such as asbestos and cigarette smoke. To effectively regulate the biological actions of exogenous and endogenous ROS, various nonenzymatic and enzymatic antioxidant defense systems are present in lung cells to provide protection and to regulate cell signaling.

However, when oxidants are produced in excess, a condition called oxidative stress occurs. Oxidative stress is defined as ROS production exceeding the capacity of the antioxidant systems to scavenge ROS, resulting from either an increased ROS generation or a decrease in antioxidant systems. Oxidative stress can damage DNA, proteins and lipids thereby promoting cell death (4). In 1956, the "free radical theory of aging" was formulated suggesting that aging (and possibly age-related disease) develop because of oxidant-induced damage (5).

Oxidative stress and IPF

Oxidative stress is thought to be implicated in a variety of chronic lung diseases including idiopathic pulmonary fibrosis (IPF). IPF is associated with an increased oxidative burden and pulmonary inflammatory cells from of IPF patients generate higher levels of ROS compared to those of healthy controls (6) and IPF patients have increased levels of $\mathrm{H}_{2} \mathrm{O}_{2}$ within their exhaled breath condensates (EBC) (7) and increased markers of oxidative damage including enhanced 8-isoprostane (2), oxidized proteins (8-10) and 8-hydroxydeoxyguanosine (11). Next to damaging macromolecules, extracellular $\mathrm{H}_{2} \mathrm{O}_{2}$ may also mediate additional fibrogenic effects by inducing apoptosis of adjacent lung epithelial cells (12). Yet, the association between oxidative stress and IPF remains controversial mostly due to the lack of our understanding how and if oxidative stress promotes the development and progression of IPF.

Studies have shown that IPF patients not only have an oxidant-imbalance due to enhanced levels of ROS, but also have a compromised antioxidant system as lung tissue from IPF patients shows decreased levels of antioxidants such as glutathione (GSH) (13, 14), superoxide dismutase 3 (15) and catalase (16). Our data further indicate that the redox-balance is disturbed in IPF as GSH as well as the total antioxidant capacity are reduced in the blood of IPF patients (chapter 3 ). Since various lines of evidence support 
these data, antioxidant therapy to reduce the oxidative burden oxidative stress in IPF, has been suggested as a therapy option. Antioxidants that also activate the redox sensitive transcription factor nuclear factor erythroid 2 related factor 2 (Nrf2) are of special interest, since they also induce various Nrf2-responsive antioxidant enzymes (17) to further protect against oxidative damage.

Based on our studies with the dietary antioxidant quercetin (chapter 3, 4) it can be concluded that quercetin demonstrated antioxidant capacities in vitro and reduced fibrotic gene expression as well as inflammatory cytokine expression in the blood of IPF patients (chapter 3). However, its effects in a mouse model of bleomycin-induced pulmonary fibrosis were only moderate. Furthermore, our results indicated that the effects of quercetin are partly mediated through the induction of Nrf2 as Nrf2 knockout mice had more pulmonary damage in response to bleomycin compared to $\mathrm{Nrf2}^{+/+}$after dietary quercetin supplementation (chapter 4).

It has been suggested before that polyphenols such as quercetin not only scavenge ROS but also form electrophilic quinones (18) that can induce Nrf2 by alkylation of critical cysteine residues of the Nrf2-regulator Kelch-like ECH-associated protein 1, also called Keap1 $(19,20)$. When Keap1 is modified through oxidation or alkylation, Nrf2 can dissociate and translocate to the nucleus leading to the activation of Nrf2-responsive genes through the antioxidant response element. As a result, a lot of studies have been focused on oxidant scavengers that are also Nrf2 activators to treat diseases with an increased oxidant burden. Interestingly, a phase 3 clinical trial with the Nrf2 activator bardoxolone in patients with stage 4 chronic kidney disease was terminated because it was not associated with beneficial health effects (21).

There are various reasons why antioxidant supplementations has not been successful until now. The biggest limitation is the physiological concentration of an antioxidant to scavenge a significant amount of ROS. Unfortunately, no antioxidant has been developed so far that reacts faster with ROS than ROS react with biomolecules to induce oxidative damage (22). Consequently, it is suggested that quercetin does not exert its beneficial effects via the scavenging of oxidants but rather through the formation of quercetin quinones. However, in high concentrations, which can luckily not be archived in vivo, quinones are toxic $(23,24)$ as they can form adducts with proteins containing thiol groups (25) thereby changing their activity. Furthermore, only because an antioxidant demonstrates oxidant scavenging abilities in vitro, it does not necessarily protect against ROS-induced damage in vivo. One of the biggest drawbacks is that we still don't know where exactly oxidants are being produced inside the cells in patients with IPF. Consequently, "overloading" the patients with antioxidants might also interfere with physiological oxidant signaling without scavenging oxidants in the right places. 
It is also important to recognize that Nrf2 translocation to the nucleus does not necessarily lead to Nrf2 activation and induction of antioxidant gene expression. A study has shown that quercetin supplementation in older mice ( $>12$ months) does not affect Nrf2 activation (26) underlining the fact that quercetin alone cannot upregulate Nrf2 in this model. Studies have suggested that Nrf2-induced antioxidant responses are declined with increasing age $(27,28)$, which is associated with simultaneous upregulation of negative Nrf2 regulators such as c-MYC and BACH-1 (29). This finding highlights the fact that inducing Nrf2 might not be enough to treat IPF as negative Nrf2 regulators might also be increased in age-related diseases. It has been shown that bleomycin-induced pulmonary fibrosis in young mice ( 2 months) results in the resolution of fibrosis after a peak around 14-21 days which is impaired in old mice (18 months) which has been linked to impaired Nrf2 signaling (30).

A deficiency in Nrf2 induction combined with upregulation of the oxidant-generating NADPH oxidase (NOX)4 has been shown to result in a redox-imbalance thereby driving pulmonary fibrosis in elderly mice (30). Various NOXes are upregulated in IPF including NOX1 (31), NOX2 (32) and NOX4 (33). NOX4 is highly upregulated in IPF, and NOX4 deficient mice are protected from bleomycin-induced pulmonary fibrosis (33) indicating that NOX4 plays an important role in the development of IPF. Indeed, it has been shown that NOX4 promotes alveolar epithelial cell death (34) and myofibroblast activation (35), thereby promoting a fibrotic environment in the lungs. Although NOX4 expression correlated with IPF disease progression (Chapter 5), it can be deducted from our results that NOX4 expression varies between IPF patients. Noticeable, not every patient had an increased expression of NOX4 (Chapter 8) indicating that also other mechanisms drive the disease. Therefore, it should be evaluated beforehand if the patient has an increased NOX4 activity. Otherwise NOX4 inhibition might not be a beneficial target for treatment and it might even interfere with physiological cell signaling.

\section{Oxidative stress versus redox regulation: a role for oxidant crosstalk?}

Over the past decade, it has become clear that simply inhibiting ROS sources or scavenging ROS might not be the solution for treating IPF as its pathology may be mediated by dysregulated redox processes rather than oxidative stress (36). Redoxdependent signaling processes include alterations of the intracellular redox state and reversible redox-mediated post-translational modifications of redox-sensitive proteins $(37,38)$. A major target of ROS is the thiol side chain of cysteine residues which is important for the regulation of protein function and serves as redox sensor. However, the molecular mechanisms by which such dysregulated redox processes contribute to IPF pathology are not fully elucidated yet.

Our results demonstrate that NOX4 can oxidize the SRC family kinase (SFK) member FYN through oxidation of one or more of its cysteine residues in a time-dependent manner in 
response to TGF- $\beta 1$ contributing to its full activity (chapter 5). This NOX4-induced activation of FYN promoted mtROS production and epithelial DNA damage responses as well as induction of profibrotic genes in bronchial epithelial cells. This finding indicates that NOX4 may promote fibrosis, at least partly, by redox-dependent activation of FYN. Other studies have also shown that oxidation of cysteine residues promotes the activity of SRC (39). However, we still need to evaluate the exact cysteine that is oxidized by NOX4 to target it directly. The most likely candidate is Cys 488 as it is conserved among all SFKs and is homologous to C487 in SRC, which has been implicated in its redox-dependent activation (40). More detailed future studies will be needed to clarify the significance of FYN sulfenylation for regulating its activity.

Another source of intracellular oxidants are the mitochondria, and IPF is characterized by mitochondrial dysfunction. Mitochondrial dysfunction is thought to enhance pathological features of IPF, in part by increasing mitochondrial ROS production and altering cellular metabolism contributing to epithelial cell death as well as profibrotic gene expression (41, 42). IPF patient derived bronchial epithelial cells have a decreased expression of genes regulating mitochondrial biogenesis as well as mitophagy (chapter 7) which might contribute to increased mtROS generation through the accumulation of dysfunctional mitochondria. Our data show that TGF- $\beta 1$ enhances mtROS which is dependent on both NOX4 and FYN (chapter 5). Intriguingly, both NOX4 (43) and FYN may be present in mitochondria, as it has been shown before that NOX4 and FYN were found to colocalize in mitochondria of cardiomyocytes (44).

Recent findings indicate reciprocal interactions between NOX enzymes and mitochondria, which affect regulation of NOX activity as well as mitochondrial function and mtROS production, and collectively promote epithelial injury and profibrotic signaling, as reviewed in (chapter 2). It has been reported that NOX4 is able to interact with mitochondrial complex I, thereby negatively regulating its activity (45). Furthermore, studies have suggested that mtROS regulate NOX4 expression in fibroblasts of IPF patients (42). Overexpression of NOX4 might have a role in mediating mitochondrial dysfunction in lung fibroblasts as NOX4 inhibition results in a decrease if mitochondrial biogenesis (46).

It is possible that targeting one oxidant source might not have a beneficial outcome, as this could result in an increase of oxidant generation from the source as a compensatory mechanism as we still don't fully understand the impact on disease progression. Furthermore, based on the idea that low levels of ROS are actually beneficial and high levels are harmful, it is almost impossible to find the right dose of an antioxidant or ROS source inhibitor to only scavenge harmful ROS. Therefore, it might be more beneficial to target redox-sensitive proteins that have been found to be involved in downstream signaling pathways, such as the SRC family kinases. 


\section{Targeting redox-sensitive proteins: the SRC family}

Since we have shown that NOX4-induced activation of the SFK member FYN is involved in fibrotic responses, we evaluated the role of SFK further. SFK have been indicated to play a role in the development of pulmonary fibrosis before and studies have shown that inhibition of SFK attenuates fibrotic lesions in experimental models of IPF $(47,48)$.

In agreement with previous studies $(47,49)$, our data show that SFK play an important role in fibroblast proliferation and activation and inhibition of SFK by saractinib reduced collagen secretion by fibroblasts (chapter 6). Bronchial epithelial cells from IPF patients demonstrated higher levels of SFK activation compared to controls, shown by phosphorylation at tyrosine 416 (chapter 7). As inhibition of SFK also reduced NOX4 expression in fibroblasts (chapter 6) as well as in epithelial cells from IPF patients (chapter 7), it can be concluded that SFK and NOX4 influence the activity of one another. However, although NOX4 correlated with IPF disease severity, the expression of the SFK member FYN was found to be significantly downregulated in lung tissue of IPF patients (chapter 5). Interestingly, it has been suggested that FYN can negatively regulate NOX4 activity through phosphorylating of Tyrosine566 in its C-terminus (44). Hence, NOX4 and FYN show a reciprocal relationship, with NOX4 being able to activate FYN, whereas FYN can in turn inhibit NOX4 through a negative feedback mechanism. Since, our findings indicate significant downregulation of FYN in IPF this might suggest that such a negative feedback is impaired in the context of IPF, thereby promoting NOX4 activation and downstream profibrotic effects. Yet, FYN might not the only SFK that is involved in IPF, since knockdown of the SFK member YES reduced TGF- $\beta 1$ induced activation of SFK as well as gene expression of COL1A1 and FN1 in fibroblasts (chapter 6). These findings emphasize the idea that different members of the SRC family mediate different aspects of IPF and might be involved in different fibrotic pathways. The SFK member YES might regulate the expression of NOX4 whereas FYN is involved in the activation of NOX4 in response to TGF$\beta 1$. Consequently, it is of interest to further evaluate the role of individual SFK members in IPF, especially since inhibition of SFK also resulted in a decreased NOX4 expression which might be independent of FYN.

Interestingly, it has been suggested that quercetin also exerts its effect by interfering with cellular signaling through the inhibition of protein kinases $(50)$ including SFK $(51,52)$. Studies have demonstrated that flavonoids, such as quercetin, can interact with SFK on a molecular level. It has been shown that quercetin can directly inhibit the kinase activity of FYN, HCK and LYN whereas other flavonoids only elicited minor effects on SFK inhibition $(53,54)$. The inhibitory effects of quercetin on SFK are probably due to the planar C-ring allowing contact between the hydrogen bonds of quercetin with residues within the catalytic side of SFK (53). These findings suggest that the effects of quercetin are possible due to the inhibition of SFK. Quercetin reduced release of pro-inflammatory cytokines in 240 
vitro and in vivo (Chapter 3 and 4) which was also observed after inhibition of SFK in fibroblasts (Chapter 6) as well as in primary human bronchial epithelial cells (Chapter 7 and 8 ).

SRC family kinases are also recognized as important metabolic regulators in mitochondria (55). Our data suggest that inhibition of SFK increases gene expression of markers involved in mitochondrial biogenesis including NRF2, PGC1 $\beta$ and ERR $\alpha$ and the mitophagy marker PINK1. Furthermore, Inhibition of SFK also increased mitochondrial biogenesis markers which was accompanied by a decrease in LDH release and BAX expression in IPF patients (chapter 7) indicating that SFK are present in the mitochondria and involved in regulating mitochondrial dynamics. Intriguingly, NOX4 has also been suggested to play a crucial role in mitochondrial biogenesis and metabolism (56) and regulating NOX4 expression through inhibition of SFK could help increase mitochondrial health in IPF patients. In conclusion, our findings suggest that targeting SFK is a promising treatment option for pulmonary fibrosis.

\section{Redox-modulatory therapy only for IPF patients with increased NOX4 expression?}

Although the paradigm of IPF has shifted from a fibroblast-activated to an epithelialdriven disease during the last decade (56), most studies are still performed only in fibroblasts. However, to investigate dysregulated pathways and effectively treat patients with IPF, it is crucial to investigate the effects of anti-fibrotic drugs on the lung epithelium as well. Therefore, we studied the current FDA-approved IPF drugs pirfenidone and nintedanib in differentiated bronchial epithelial cells of IPF patients and compared their responses to the SFK inhibitor saracatinib. In this pilot study, we demonstrated that bronchial epithelial cells from the epithelial lining fluid can be differentiated into a complete bronchial epithelium, consisting of ciliated epithelial, basal, goblet and club cells. Recently, it has been suggested that a shift towards a more personalized approach in IPF could be a realistic goal to improve treatment of affected patients (57-59). Indeed, our pilot data support that individual IPF patients responded differently to the antifibrotic drugs, even only a small number of IPF patients was included in our study $(n=4)$ highlighting how heterogeneous the disease is.

To maximize the outcome of personalized medicine, subgroups of IPF patients have to be identified first to develop targeted therapies as IPF is a very heterogeneous disease. Possible traits for defining such subgroups could be the redox status and or the inflammatory status of patients with IPF. The redox status plays an important role in the disease, however, not all patients might have an increased oxidant burden, which can also be deducted from the spread in NOX4 and AOX expression observed in the present study. This might also explain why antioxidant therapy has failed to be generally effective in IPF 
thus far. Instead, patients with increased NOX4 expression might benefit from treatment redox-modulatory therapy whereas patients without an altered redox balance might rather benefit from an alternative treatment approach. Another way of phenotyping IPF patients could be by inflammatory status as our data have indicated that some but not all patients demonstrate significantly elevated levels of pro-inflammatory cytokines and that saracatinib can reduces IL-8 secretion in those patients.

In conclusion, this pilot study indicates that responsiveness to treatment varies considerably between individual IPF patients and that IPF patients would benefit from personalized treatment to maximize the therapy response (chapter 8). To achieve this goal, markers for the prediction of treatment need to be developed and such markers could include NOX4 activity, antioxidant gene expression, inflammatory status but also SRC family kinase activation. 


\section{Directions for further research}

The evidence for the involvement of ROS in IPF is growing, however, it is still unclear whether ROS actively promote the progression of the disease and/or dysregulate cellular signaling through oxidative protein modifications. It is not known which specific downstream targets are oxidized by NOX4 and mitochondrial ROS and if these contribute to $A E C$ apoptosis and fibroblast activation. Future research should therefore focus on redox signaling, e.g. reversible modifications of proteins, instead of solely targeting the redox-balance by inhibiting ROS sources or enhancing antioxidant scavenging potential. We have already identified that reversible oxidation of thiol groups is involved in TGF- $\beta 1$ induced oxidant production. However, we only focused on oxidation of thiol groups within cysteine residues, and it would also be of interest to investigate other modifications such as disulfide bonds and glutathionylation.

To this end, the location of oxidant sources has to be identified as well. For instance, it has been indicated that NOX4 may be localized to mitochondria (60) and that mitochondria and NOX4 exert reciprocal interactions (61). However, studies addressing these questions are limited mainly because no crystal structure of NOX4 exists and therefore specific antibodies targeting NOX4 are not commercially available. Secondly, it is still generally thought that NOX4 is constitutively active and regulated through its gene expression. Emerging evidence, however, suggests that NOX4 expression can be inhibited through scavenging of mitochondrial ROS (42), indicating crosstalk between these oxidant sources. Furthermore, our results indicate that NOX4 expression can be reduced by inhibiting SFK. Indeed, it has been suggested before that kinases can phosphorylate NOX subunits thereby affecting their activity (62). Therefore, future studies should also aim to unravel the molecular redox mechanisms underlying such interactions.

Furthermore, we have described that NOX4 plays an important role in the activation of SFK and that SFK are central in the induction of fibrotic genes in epithelial cells as well as fibroblasts. Inhibition of SFK in bronchial epithelial cells of IPF patients reduced NOX4 expression and inflammation indicating that SFK are a potential target in the treatment of pulmonary fibrosis but also suggesting a feed-forward-loop between NOX4 and SFK. For this purpose, research should also focus on finding specific targets of saracatinib. Our data also suggest that saracatinib targets different members in different cell types therefore it might be more effective to target one specific members of the SFK family in one cell type instead of targeting a class of proteins. However, on the other hand, an inhibitor of the whole family might be also beneficial as it targets various members but can thereby also exert unwanted side effects. Intriguingly, recently a preclinical and phase $1 \mathrm{~b} / 2 \mathrm{a}$ clinical trial investigating the benefits of saracatinib as treatment strategy for IPF, has been funded by the NIH (\#1UG3TR002445-01). 
Lastly, it is of importance to study pulmonary fibrosis in the right models. Most of our in vitro studies have been performed in bronchial epithelial cells but IPF primarily impacts the alveolar epithelium (63). However, primary alveolar epithelial cell lines are more difficult to obtain compared to bronchial epithelial cells. It is not yet achievable to obtain alveolar epithelial cells from patients with lung diseases in a non-invasive way. Furthermore, bronchial epithelial cells have also been indicated in fibrotic remodeling as it has been suggested that injury to the bronchial epithelium also promotes the development of pulmonary fibrosis (64) but studies are limited. A better way to study fibrotic responses would be by using three-dimensional tissue cultures which also allow cellular crosstalk. The use of organoids might provide a new way to study IPF and predict treatment responses in individual IPF patients. Bronchiolar and alveolar organoids grown from pluripotent stem cells isolated from blood of patients $(65,66)$ mimic the lung structure very accurately and might be used to identify changes to specific cell types early in the progression of IPF. Additionally, such specific organoid systems could be used to understand how antifibrotic drugs work and to identify new targets for personalized treatment.

In conclusion, new treatment strategies for IPF are desperately needed. Although the evidence regarding NOX4-mediated pathways is growing, we still know relatively little about the precise molecular mechanisms and its targets. Therefore, future research should mainly focus on the identification of such molecular redox mechanisms to develop more targeted redox-based therapies, especially for IPF patients with an increased oxidative burden. 


\section{Implications}

IPF is generally diagnosed in the sixth decade of life, and aging is now recognized as one of the strongest risk factors for IPF (67). It is suggested that the incidence of IPF is increasing every year by $11 \%$ (68) probably due to the increase of elderly people in our society as well as the improvements in diagnosis. IPF dramatically reduces the quality of life for the patients as the breathlessness they are experiencing limits their daily activities. Moreover, the oxygen shortage often leads to lethargy, concentration problems and sometimes depressive moods. However, only minimal advances have been made in the last years to improve IPF outcome and treatment. IPF is still a fatal disease with a median survival of less than 4 years (69). Currently, there are two IPF drugs available, but these drugs are not effective in all patients and there is limited information available regarding which patients should be treated with which drug. The only cure for IPF is lung transplantation. Consequently, there is an urgent need to improve the current and develop new therapy strategies.

Since IPF is such a devastating disease, more effort must be made towards the understanding of the underlying mechanisms of IPF and their contribution to disease pathogenesis. IPF has been associated with an increase in protein oxidation $(70,71)$. However, only a few studies have addressed the role of protein oxidation in fibrotic signaling, although the disease is associated with an increased oxidative burden. Our studies support the idea that reversible oxidation of proteins is important for fibrotic signaling which could provide a way for more targeted therapies that do not interfere with physiological oxidant signaling as oxidant sources are not being inhibited. Our data also highlight that IPF is a very heterogeneous disease and that not every IPF patient suffers from an increased oxidative burden associated with increased NOX4 expression.

In conclusion, this thesis contributes to the understanding of the importance of redox regulation in IPF. Our findings indicate that inhibition of redox-sensitive proteins such as the SRC family kinases could provide new directions for treatment for patients with IPF. Additionally, our data indicate the urge to work more towards personalized approaches as IPF is such a heterogeneous disease that the idea that one drugs fits all will not decrease the high mortality associated with the disease. To achieve this next step, we need stratification of patient subgroups as well as markers to predict therapy outcome in these different groups. Since biomarkers are difficult to obtain for such a heterogeneous disease, an alternative approach would be the testing of different drugs on e.g. 3-D cell cultures that can be obtained through isolation of pluripotent stem cells from blood of patients before therapy to maximize the treatment response through personalized medicine. 


\section{References}

1. Kinnula VL, Fattman CL, Tan RJ, Oury TD. Oxidative stress in pulmonary fibrosis: a possible role for redox modulatory therapy. Am J Respir Crit Care Med. 2005;172(4):417-22.

2. Montuschi P, Ciabattoni G, Paredi P, Pantelidis P, du Bois RM, Kharitonov SA, et al. 8Isoprostane as a biomarker of oxidative stress in interstitial lung diseases. American journal of respiratory and critical care medicine. 1998;158(5 Pt 1):1524-7.

3. Thannickal VJ, Fanburg BL. Reactive oxygen species in cell signaling. Am J Physiol Lung Cell Mol Physiol. 2000;279(6):L1005-28.

4. Freeman BA, Crapo JD. Biology of disease: free radicals and tissue injury. Lab Invest. 1982;47(5):412-26.

5. Harman D. Aging: a theory based on free radical and radiation chemistry. J Gerontol. 1956;11(3):298-300.

6. Cantin AM, North SL, Fells GA, Hubbard RC, Crystal RG. Oxidant-mediated epithelial cell injury in idiopathic pulmonary fibrosis. The Journal of clinical investigation. 1987;79(6):1665-73.

7. Psathakis K, Mermigkis D, Papatheodorou G, Loukides S, Panagou P, Polychronopoulos V, et al. Exhaled markers of oxidative stress in idiopathic pulmonary fibrosis. Eur J Clin Invest. 2006;36(5):362-7.

8. Lenz AG, Costabel U, Maier KL. Oxidized BAL fluid proteins in patients with interstitial lung diseases. The European respiratory journal. 1996;9(2):307-12.

9. Rottoli P, Magi B, Cianti R, Bargagli E, Vagaggini C, Nikiforakis N, et al. Carbonylated proteins in bronchoalveolar lavage of patients with sarcoidosis, pulmonary fibrosis associated with systemic sclerosis and idiopathic pulmonary fibrosis. Proteomics. 2005;5(10):2612-8.

10. Saleh D, Barnes PJ, Giaid A. Increased production of the potent oxidant peroxynitrite in the lungs of patients with idiopathic pulmonary fibrosis. American journal of respiratory and critical care medicine. 1997;155(5):1763-9.

11. Kuwano K, Nakashima N, Inoshima I, Hagimoto N, Fujita M, Yoshimi M, et al. Oxidative stress in lung epithelial cells from patients with idiopathic interstitial pneumonias. The European respiratory journal. 2003;21(2):232-40.

12. Waghray M, Cui Z, Horowitz JC, Subramanian IM, Martinez FJ, Toews GB, et al. Hydrogen peroxide is a diffusible paracrine signal for the induction of epithelial cell death by activated myofibroblasts. FASEB J. 2005;19(7):854-6.

13. Cantin AM, Hubbard RC, Crystal RG. Glutathione deficiency in the epithelial lining fluid of the lower respiratory tract in idiopathic pulmonary fibrosis. Am Rev Respir Dis. 1989;139(2):370-2.

14. Meyer A, Buhl R, Magnussen $\mathrm{H}$. The effect of oral N-acetylcysteine on lung glutathione levels in idiopathic pulmonary fibrosis. The European respiratory journal. 1994;7(3):431-6.

15. Kinnula VL, Hodgson UA, Lakari EK, Tan RJ, Sormunen RT, Soini YM, et al. Extracellular superoxide dismutase has a highly specific localization in idiopathic pulmonary fibrosis/usual interstitial pneumonia. Histopathology. 2006;49(1):66-74.

16. Odajima N, Betsuyaku T, Nagai K, Moriyama C, Wang DH, Takigawa T, et al. The role of catalase in pulmonary fibrosis. Respir Res. 2010;11:183.

17. Cheresh P, Kim SJ, Tulasiram S, Kamp DW. Oxidative stress and pulmonary fibrosis. Biochim Biophys Acta. 2013;1832(7):1028-40.

18. Boots AW, Haenen GR, Bast A. Health effects of quercetin: from antioxidant to nutraceutical. Eur J Pharmacol. 2008;585(2-3):325-37.

19. Luo Y, Eggler AL, Liu D, Liu G, Mesecar AD, van Breemen RB. Sites of alkylation of human Keap1 by natural chemoprevention agents. J Am Soc Mass Spectrom. 2007;18(12):2226-32.

20. Magesh S, Chen Y, Hu L. Small molecule modulators of Keap1-Nrf2-ARE pathway as potential preventive and therapeutic agents. Med Res Rev. 2012;32(4):687-726. 
21. de Zeeuw D, Akizawa T, Audhya P, Bakris GL, Chin M, Christ-Schmidt H, et al. Bardoxolone methyl in type 2 diabetes and stage 4 chronic kidney disease. N Engl J Med. 2013;369(26):2492-503. 22. Forman HJ, Davies KJ, Ursini F. How do nutritional antioxidants really work: nucleophilic tone and para-hormesis versus free radical scavenging in vivo. Free Radic Biol Med. 2014;66:24-35. 23. Metodiewa D, Jaiswal AK, Cenas N, Dickancaite E, Segura-Aguilar J. Quercetin may act as a cytotoxic prooxidant after its metabolic activation to semiquinone and quinoidal product. Free Radic Biol Med. 1999;26(1-2):107-16.

24. Wang X, Thomas B, Sachdeva R, Arterburn L, Frye L, Hatcher PG, et al. Mechanism of arylating quinone toxicity involving Michael adduct formation and induction of endoplasmic reticulum stress. Proc Natl Acad Sci U S A. 2006;103(10):3604-9.

25. Boots AW, Kubben N, Haenen GR, Bast A. Oxidized quercetin reacts with thiols rather than with ascorbate: implication for quercetin supplementation. Biochem Biophys Res Commun. 2003;308(3):560-5.

26. Hohmann MS, Habiel DM, Coelho AL, Verri WA, Jr., Hogaboam CM. Quercetin Enhances Ligand-induced Apoptosis in Senescent Idiopathic Pulmonary Fibrosis Fibroblasts and Reduces Lung Fibrosis In Vivo. Am J Respir Cell Mol Biol. 2019;60(1):28-40.

27. Zhang $\mathrm{H}$, Davies KJA, Forman HJ. Oxidative stress response and Nrf2 signaling in aging. Free Radic Biol Med. 2015;88(Pt B):314-36.

28. Swamy SM, Rajasekaran NS, Thannickal VJ. Nuclear Factor-Erythroid-2-Related Factor 2 in Aging and Lung Fibrosis. Am J Pathol. 2016;186(7):1712-23.

29. Zhou L, Zhang H, Davies KJA, Forman HJ. Aging-related decline in the induction of Nrf2regulated antioxidant genes in human bronchial epithelial cells. Redox biology. 2018;14:35-40.

30. Hecker L, Logsdon NJ, Kurundkar D, Kurundkar A, Bernard K, Hock T, et al. Reversal of persistent fibrosis in aging by targeting Nox4-Nrf2 redox imbalance. Sci Transl Med. 2014;6(231):231ra47.

31. Choi SH, Kim M, Lee HJ, Kim EH, Kim CH, Lee YJ. Effects of NOX1 on fibroblastic changes of endothelial cells in radiationinduced pulmonary fibrosis. Mol Med Rep. 2016;13(5):4135-42.

32. Manoury B, Nenan S, Leclerc O, Guenon I, Boichot E, Planquois JM, et al. The absence of reactive oxygen species production protects mice against bleomycin-induced pulmonary fibrosis. Respir Res. 2005;6:11.

33. Hecker L, Vittal R, Jones T, Jagirdar R, Luckhardt TR, Horowitz JC, et al. NADPH oxidase-4 mediates myofibroblast activation and fibrogenic responses to lung injury. Nat Med.

2009;15(9):1077-81.

34. Carnesecchi S, Deffert C, Donati Y, Basset O, Hinz B, Preynat-Seauve O, et al. A key role for NOX4 in epithelial cell death during development of lung fibrosis. Antioxidants \& redox signaling. 2011;15(3):607-19.

35. Amara N, Goven D, Prost F, Muloway R, Crestani B, Boczkowski J. NOX4/NADPH oxidase expression is increased in pulmonary fibroblasts from patients with idiopathic pulmonary fibrosis and mediates TGFbeta1-induced fibroblast differentiation into myofibroblasts. Thorax. 2010;65(8):733-8.

36. van der Vliet A, Janssen-Heininger YMW, Anathy V. Oxidative stress in chronic lung disease: From mitochondrial dysfunction to dysregulated redox signaling. Mol Aspects Med. 2018.

37. Ray PD, Huang BW, Tsuji Y. Reactive oxygen species (ROS) homeostasis and redox regulation in cellular signaling. Cell Signal. 2012;24(5):981-90.

38. Droge W. Free radicals in the physiological control of cell function. Physiological reviews. 2002;82(1):47-95.

39. Heppner DE, Dustin CM, Liao C, Hristova M, Veith C, Little AC, et al. Direct cysteine sulfenylation drives activation of the Src kinase. Nat Commun. 2018;9(1):4522. 
40. Giannoni E, Buricchi F, Raugei G, Ramponi G, Chiarugi P. Intracellular reactive oxygen species activate Src tyrosine kinase during cell adhesion and anchorage-dependent cell growth. Mol Cell Biol. 2005;25(15):6391-403.

41. Patel AS, Song JW, Chu SG, Mizumura K, Osorio JC, Shi Y, et al. Epithelial cell mitochondrial dysfunction and PINK1 are induced by transforming growth factor-beta1 in pulmonary fibrosis. PloS one. 2015;10(3):e0121246.

42. Jain M, Rivera S, Monclus EA, Synenki L, Zirk A, Eisenbart J, et al. Mitochondrial reactive oxygen species regulate transforming growth factor-beta signaling. J Biol Chem. 2013;288(2):770-7.

43. Bernard K, Hecker L, Luckhardt TR, Cheng G, Thannickal VJ. NADPH oxidases in lung health and disease. Antioxidants \& redox signaling. 2014;20(17):2838-53.

44. Matsushima S, Kuroda J, Zhai P, Liu T, Ikeda S, Nagarajan N, et al. Tyrosine kinase FYN negatively regulates NOX4 in cardiac remodeling. The Journal of clinical investigation. 2016;126(9):3403-16.

45. Koziel R, Pircher H, Kratochwil M, Lener B, Hermann M, Dencher NA, et al. Mitochondrial respiratory chain complex I is inactivated by NADPH oxidase Nox4. Biochem J. 2013;452(2):231-9.

46. Bernard K, Logsdon NJ, Miguel V, Benavides GA, Zhang J, Carter AB, et al. NADPH Oxidase 4 (Nox4) Suppresses Mitochondrial Biogenesis and Bioenergetics in Lung Fibroblasts via a Nuclear Factor Erythroid-derived 2-like 2 (Nrf2)-dependent Pathway. J Biol Chem. 2017;292(7):3029-38.

47. Hu M, Che P, Han X, Cai GQ, Liu G, Antony V, et al. Therapeutic targeting of SRC kinase in myofibroblast differentiation and pulmonary fibrosis. The Journal of pharmacology and experimental therapeutics. 2014;351(1):87-95.

48. Lu YY, Zhao XK, Yu L, Qi F, Zhai B, Gao CQ, et al. Interaction of Src and Alpha-V Integrin Regulates Fibroblast Migration and Modulates Lung Fibrosis in A Preclinical Model of Lung Fibrosis. Sci Rep. 2017;7:46357.

49. Abdalla M, Thompson L, Gurley E, Burke S, Ujjin J, Newsome R, et al. Dasatinib inhibits TGFbeta-induced myofibroblast differentiation through Src-SRF Pathway. Eur J Pharmacol. 2015;769:134-42.

50. Williams RJ, Spencer JP, Rice-Evans C. Flavonoids: antioxidants or signalling molecules? Free Radic Biol Med. 2004;36(7):838-49.

51. Houseman BT, Huh JH, Kron SJ, Mrksich M. Peptide chips for the quantitative evaluation of protein kinase activity. Nat Biotechnol. 2002;20(3):270-4.

52. Huang YT, Hwang JJ, Lee PP, Ke FC, Huang JH, Huang $\mathrm{CJ}$, et al. Effects of luteolin and quercetin, inhibitors of tyrosine kinase, on cell growth and metastasis-associated properties in A431 cells overexpressing epidermal growth factor receptor. Br J Pharmacol. 1999;128(5):999-1010.

53. Wright B, Watson KA, McGuffin LJ, Lovegrove JA, Gibbins JM. GRID and docking analyses reveal a molecular basis for flavonoid inhibition of Src family kinase activity. J Nutr Biochem. 2015;26(11):1156-65.

54. Wright B, Moraes LA, Kemp CF, Mullen W, Crozier A, Lovegrove JA, et al. A structural basis for the inhibition of collagen-stimulated platelet function by quercetin and structurally related flavonoids. Br J Pharmacol. 2010;159(6):1312-25.

55. Ogura M, Yamaki J, Homma MK, Homma Y. Mitochondrial c-Src regulates cell survival through phosphorylation of respiratory chain components. The Biochemical journal. 2012;447(2):281-9.

56. Bernard K, Logsdon NJ, Miguel V, Benavides GA, Zhang J, Carter AB, et al. NADPH Oxidase 4 (Nox4) Suppresses Mitochondrial Biogenesis and Bioenergetics in Lung Fibroblasts via a Nuclear Factor Erythroid-derived 2-like 2 (Nrf2)-dependent Pathway. J Biol Chem. 2017;292(7):3029-38. 57. Selman M, King TE, Pardo A, American Thoracic S, European Respiratory S, American College of Chest P. Idiopathic pulmonary fibrosis: prevailing and evolving hypotheses about its pathogenesis and implications for therapy. Ann Intern Med. 2001;134(2):136-51. 
58. Spagnolo $P$, Oldham JM, Jones MG, Lee JS. Personalized medicine in interstitial lung diseases. Curr Opin Pulm Med. 2017;23(3):231-6.

59. Sato S, Kolb MRJ. Personalised medicine for IPF: getting closer, but not there yet. Lancet Respir Med. 2017;5(11):836-7.

60. Thannickal VJ, Antony VB. Is personalized medicine a realistic goal in idiopathic pulmonary fibrosis? Expert Rev Respir Med. 2018;12(6):441-3.

61. Graham KA, Kulawiec M, Owens KM, Li X, Desouki MM, Chandra D, et al. NADPH oxidase 4 is an oncoprotein localized to mitochondria. Cancer Biol Ther. 2010;10(3):223-31.

62. Chowdhury AK, Watkins T, Parinandi NL, Saatian B, Kleinberg ME, Usatyuk PV, et al. Srcmediated tyrosine phosphorylation of p47phox in hyperoxia-induced activation of NADPH oxidase and generation of reactive oxygen species in lung endothelial cells. J Biol Chem.

2005;280(21):20700-11.

63. Martinez FJ, Collard HR, Pardo A, Raghu G, Richeldi L, Selman M, et al. Idiopathic pulmonary fibrosis. Nat Rev Dis Primers. 2017;3:17074.

64. Evans CM, Fingerlin TE, Schwarz MI, Lynch D, Kurche J, Warg L, et al. Idiopathic Pulmonary Fibrosis: A Genetic Disease That Involves Mucociliary Dysfunction of the Peripheral Airways. Physiol Rev. 2016;96(4):1567-91.

65. Barkauskas CE, Chung MI, Fioret B, Gao X, Katsura H, Hogan BL. Lung organoids: current uses and future promise. Development. 2017;144(6):986-97.

66. Yamamoto M, Wilting J, Abe H, Murakami G, Rodriguez-Vazquez JF, Abe SI. Development of the pulmonary pleura with special reference to the lung surface morphology: a study using human fetuses. Anat Cell Biol. 2018;51(3):150-7.

67. Pardo A, Selman M. Lung Fibroblasts, Aging, and Idiopathic Pulmonary Fibrosis. Annals of the American Thoracic Society. 2016;13(Supplement_5):S417-S21.

68. Gribbin J, Hubbard RB, Le Jeune I, Smith CJ, West J, Tata LJ. Incidence and mortality of idiopathic pulmonary fibrosis and sarcoidosis in the UK. Thorax. 2006;61(11):980-5.

69. Raghu G, Chen SY, Yeh WS, Maroni B, Li Q, Lee YC, et al. Idiopathic pulmonary fibrosis in US Medicare beneficiaries aged 65 years and older: incidence, prevalence, and survival, 2001-11. Lancet Respir Med. 2014;2(7):566-72.

70. Hipkiss AR. Accumulation of altered proteins and ageing: causes and effects. Exp Gerontol. 2006;41(5):464-73.

71. Pennathur S, Vivekanandan-Giri A, Locy ML, Kulkarni T, Zhi D, Zeng L, et al. Oxidative Modifications of Protein Tyrosyl Residues Are Increased in Plasma of Human Subjects with Interstitial Lung Disease. Am J Respir Crit Care Med. 2016;193(8):861-8. 



\section{Addendum}

Samenvatting

\section{Zusammenfassung}

Valorization

Acknowledgements/ Dankwoord/ Danksagungen

List of publications

Curriculum Vitae 

Samenvatting 
Idiopathische longfibrose (IPF) is een chronische longziekte met een gemiddelde levensverwachting van slechts drie jaar na diganose. Longfibroe wordt gekarakteriseerd door de vorming van littekenweefsel in de longen dat tot zogeheten long falen leidt. In Nederland wordt bij gemiddeld 1000 mensen per jaar longfibrose ontdekt. De ziekte wordt gekenmerkt door een overproductie van schadelijke reactieve zuurstof radicalen (RZS) en verhoogde markers van oxidatieve schade zijn aangetoond in de longen van IPF patienten. Desondanks zijn antioxidant-therapieën niet bewezen effectief bij de behandeling van IPF en de rol van oxidatieve stress in de ontwikkeling van de ziekte is nog steeds niet helemaal duidelijk. Het doel van dit proefschrift was om het belang van oxidant regulatie bij pulmonale fibrose te onderzoeken. Hiertoe werd 1) de rol van RZS bestudeerd door RZS weg te vangen met het antioxidant quercetine, 2) de rol van RZS geproduceerd door het enzym NADPH-oxidase NOX4 bij de activatie van SRC kinasen onderzocht en 3 ) het effect van SRC kinasen (SFK) inhibitie om specifieke kenmerken van IPF te verminderen geëvalueerd.

Hoofdstuk 2 omvat een literatuuroverzicht over oxidant regulatie in longfibrose. IPF wordt gekenmerkt door terugkerende beschadigingen van de epitheliale long cellen die resulteren in afwijkende wondgenezingreacties en uiteindelijk tot overmatige afzetting van collageen in de long. In dit hoffdstuk is de huidige kennis met betrekking tot verschillende aspecten van RZS-disbalans in de ontwikkeling van IPF samengevat. Speciale nadruk is gelegd op het belang van disregulatie van NOX-activering, mitochondriale disfunctie en de communicatie tussen NOX4 en mitochondriën. Wij concluderen dat selectieve remming van specifieke NOX-isovormen aangrijpspunt kan zijn voor de behandeling van IPF. Deze conclusie is met name relevant in het licht van recente studies die aantonen dat redoxveranderingen geïnduceerd kunnen worden door mitochondriale interacties met NOX enzymen.

In Hoofdstuk 3 hebben we de mogelijk beschermende effecten van het antioxidant quercetine op oxidant productie en pro-inflammatoire cytokine secretie in humane bronchiale epitheel cellen en ex vivo in het bloed van IPF patiënten onderzocht. Quercetine verlaagde de door bleomycine-geïnduceerde RZS-productie, terwijl dit antioxidant een verhoging veroorzaakte van de Nrf2 activiteit en de afgifte van inflammatoire cytokines in vitro en ex vivo in het bloed van IPF patiënten werd verminderd. Eén week met quercetine suppletie bij muizen verhoogdede concentratie van quercetine in het bloedplasma, maar niet in de longen, en verhoogde enigszins de pulmonale expressie van Nrf2-responsieve genen (hoofdstuk 4). Na blootstelling aan bleomycine vertoonden quercetine gevoede wildtype muizen verminderde expressie van collageen en fibronectine evenals verminderde pulmonaire inflammatoire laesies. Bovendien verminderde quercetine de pulmonaire genexpressie van TNFa en KC, maar niet hun plasma levels, en verminderde malondialdehyde-dG DNA-adducten in de long. In 
Nrf2 knockout dieren werd geen verbetering van quercetine op ontstekingslaesies in de long waargenomen bij blootstelling aan bleomycine, dat suggereert dat quercetine beschermt tegen bleomycine-geïnduceerde longschade in de wildtype muizen biedt via Nrf2. Quercetine suppletie kon de bleomycine-geïnduceerde schade echter niet volledig ongedaan maken, hetgeen erop wijst dat quercetine toevoeging aan het dieet niet voldoende is om de ontwikkeling van pulmonaire fibrosee tegen te gaan.

Een belangrijke bron van RZS in de longen is het enzym NADPH-oxidase (NOX) 4. De eiwitten die door de RZS geproduceerd door NOX4 worden gemodificeerd zijn grotendeels nog onbekend alsook het effect hiervan. In hoofdstuk $\mathbf{5}$ is de reactie van TGF- $\beta 1$ op humane bronchiale epitheelcelen bestudeerd en het bleek dat NOX4 het SFK-familielid FYN oxideerde en daardoor activeerde. Deze actiavie leidt tot een verhoodge RZS production, fibrotische gene expressie en DNA schade. Wanneer FYN werd geremd door de pharmacologische remmer PP2 of door het gebruik van siRNA, verminderde het de mitochondriale RZS-productie, wat leidde tot vermindering van DNA-schade en expressie van fibronectine. Inhibitie van SFK door de farmacologische remmer saracatinib verminderde de activatie van fibroblasten en de productie van RZS-en inflammatoire cytokines door deze cellen, zoals gepresenteerd in hoofdstuk 6. Opvallend is dat TGF- $\beta 1$ blootstelling in fibroblasten een ander SFK-familielid, te weten YES, activeerde dat bijdroeg aan de expressie van extracellulaire matrixgenen. Verder was de TGFgeïnduceerde toename in NOX4-expressie verminderd in cellen waarin YES was geblokkeerd. Deze observatie geeft aan dit SFK-familielid fibroblasten activatie bevordert, vermoedelijk via inductie van NOX4.

In hoofdstuk 7 hebben we aangetoond dat bronchiale epitheelcellen van IPF-patiënten een verhoogde activatie van SFK vertonen. Remming van deze SFK door saracatinib verlaagde markers van mitochondriale dysfunctie door het verhogen van de expressie van genen geassocieerd met mitochondriale biogenese en mitofagie. Bovendien verminderde SFK remming de afgifte van $L D H$, wat wijst op celbeschadiging, en de genexpressie van het pro-apoptotische BAX alsook het enzym NOX4. In gedifferentieerde bronchiale epitheelcellen van IPF-patienten was de expressie van NOX4 licht verhoogd in vergelijking met epitheelcellen van controles, maar deze verschillen op individueel niveau (hoofdstuk 8). Op dit moment zijn er geen volledig effectieve behandelingsmogelijkheden voor IPF. Er zijn wel twee medicijnen die de achteruitgang van de ziekte kunnen remmen, te weten pirfenidone en nintedanib, maar deze werken niet bij alle patiënten. Een intrigerende bevinding was dat bij patiënten die een hogere NOX4-expressie hadden in hun epitheelcellen, pirfenidon een verhoogde expressie van antioxidant genen induceerde. Een andere interessante vondst was de significante verhoging van het pro-inflammatoire IL-8 in bronchiale epitheel cellen afkomstig van IPF patiënten die significant verlaagd werd doorbehandeling met de farmacologische SFK remmer saracatinib. Na vergelijking van de 
effecten van de reguliere IPF geneesmiddelen pirfenidon en nintedanib met die van de SFK remmer saracatinib in gedifferentieerde bronchiale epitheelcellen van IPF patienten stellen we voor dat deze patiënten zouden kunnen profiteren van een gepersonaliseerde behandeling. Deze aanbeveling is gebaseerd op de bevinding dat de respons op de verschillende geneesmiddelen sterk varieerde tussen individuele patiënten en afhankelijk was van onder meer hun redox en inflammatoire status.

In het laatste hoofdstuk van dit proefschrift (hoofdstuk 9) worden de belangrijkste bevindingen samengevat en besproken. Concluderend kan er gesteld worden dat dit proefschrift bijdraagt aan meerbegrip van redox-regulatie in long fibrose. Onze bevindingen geven aan dat modulatie van redox-gevoelige eiwitten zoals de SRC kinasen nieuwe aanwijzingen kan geven voor de behandeling van patiënten met IPF. Bovendien geven onze resultaten aan dat er meer aandacht moet worden besteed aan gepersonaliseerde behandeling. Dit past bij het beelddat IPF een erg heterogene ziekte is waarbij één medicijn voor iedereen niet afdoende zal zijn om de hoge sterfte als gevolg van deze ziekte te verminderen. Om deze volgende stap te bereiken hebben we stratificatie van patiëntensubgroepen nodig als ook specifieke markers om vervolgens de therapieresultaten in deze verschillende groepen te voorspellen en op te volgen. Omdat algemeen voorspellende biomarkers moeilijk te verkrijgen zijn voor een dergelijke heterogene ziekte, zijn alternatieve benaderingen nodig. Een dergelijke beandering zou het testen van verschillende geneesmiddelen op bronchiale epitheelcellen van IPF patienten zijn., verkegen tijdens bronchoscopie, zodat vóór aanvang van de therapie de uitkomst van de behandeling geoptimalisserd kan worden. 


\section{Zusammenfassung}


Idiopathische Lungenfibrose (IPF) ist eine chronische Lungenerkrankung mit einer Lebenserwartung von nur drei Jahren nach der Diagnose. In den Niederlanden wird eine Lungenfibrose bei durchschnittlich 1.000 Menschen pro Jahr diagnostiziert. Wissenschaftler haben herausgefunden, dass Lungenfibrose durch eine Schädigung der Epithelzellen in der Lunge entsteht. Diese Schädigung sorgt für eine vermehrte Freisetzung von Entzündungsmediatoren und der Bildung von Bindegewebe durch Fibroblasten, was die Sauerstoffaufnahme zwischen den Lungenbläschen ins Blut erschwert. Diese Narbenbildung in der Lunge führt langfristig zu einer Verschlechterung der Lungenfunktion und letztendich zu Lungenversagen. Dieser Prozess ist durch eine Überproduktion von schädlichen freien Sauerstoffradikalen gekennzeichnet und IPF-Patienten weisen erhöhte Marker für oxidative Schäden in der Lunge auf. Reaktive Sauerstoffradikale entstehen in den Mitochondrien als Nebenprodukt der Zellatmung, werden aber auch von speziellen Enzymen im Körper, den sogenannten NADPH Oxidasen (NOX), produziert. Sauerstoffradikale regulieren wichtige Prozesse im Körper, können jedoch in zu hohen Konzentrationen die Zelle schädigen und zu „oxidativem Stress“ führen. Deshalb haben Antioxidantien eine große Bedeutung als Radikalfänger in der Lunge und regulieren die Konzentration von Sauerstoffradikalen. Trotz der Schlüsselrolle von Sauerstoffradikalen haben sich Therapien mit Antioxidantien bei der Behandlung von IPF nicht als wirksam erwiesen. Das hängt damit zusammen, dass die Rolle von oxidativem Stress bei der Entstehung der Krankheit noch nicht klar definiert und die Regulierung von Sauerstoffradikalen sehr schwierig ist, da sie im Körper auch eine wichtige Rolle als Signalmoleküle einnehmen.

Ziel dieser Doktorarbeit ist es, die Bedeutung von Sauerstoffradikalen bei der Entstehung von Lungenfibrose genauer zu untersuchen. Zu diesem Zweck wird 1) die Rolle von reaktiven Sauerstoffspezies (ROS) in einem Zell- und Mäusemodell für Lungenfibrose bestimmt, indem ROS mit dem Antioxidanten Quercetin neutralisiert wird. 2) Danach wird die Rolle von Sauerstoffradikalen, die spezifisch durch die NADPH-Oxidase NOX4 entstehen, und deren Rolle bei der Aktivierung von SRC-Kinasen in einem Zellmodell untersucht. 3) Die Rolle von SRC-Kinasen Inhibitoren wird ausgewertet, um spezifische Merkmale von IPF in primären Zellen von IPF-Patienten zu reduzieren.

Wie in Kapitel 2 beschrieben, zeichnet sich IPF durch eine wiederkehrende Verletzung der Lungen-Epithelzellen aus, die zu einer abnormalen Wundheilungsreaktionen führt und schließlich eine übermäßige Bindegewebeablagerung in der Lunge bewirkt. Darüber hinaus wird das aktuelle Wissen in Bezug auf die verschiedenen Aspekte des ROS-Ungleichgewichts in Zusammenhang mit IPF und den potentiellen Rollen bei der Krankheitsentwicklung zusammengefasst. Der Schwerpunkt dieser Literaturübersicht liegt jedoch auf der Bedeutung von unangemessener NOX-Aktivierung, dem Effekt von einer mitochondrialen Dysfunktion und der Rolle von Oxidantenkommunikation zwischen NOX4 
und den Mitochondrien. Insgesamt deuten viele Studien darauf hin, dass das selektive Targeting spezifischer NOX-Isoformen und/oder die Produktion von mitochondrialen ROS für die Bekämpfung von IPF vorteilhaft sein können.

In Kapitel 3 werden die möglichen schützenden Wirkungen des Antioxidanten Quercetin auf die Produktion von Sauerstoffradikalen und Entzündungsmediatoren in humanen bronchialen Epithelzellen und im Blut von IPF-Patienten untersucht. Quercetin gehört zur Familie der Polyphenole und Flavaniode, welche in hohen Konzentrationen in Obst und Gemüse gefunden werden können. In Zellen reduziert Quercetin die durch Bleomycin, ein häufig genutztes Modell für die Initiierung von Fibrose, induzierte ROS-Produktion und erhöht gleichzeitig die Nrf2-Aktivität. Nrf2 ist ein wichtiger Transkriptionsfaktor, der durch ROS sowie durch Quercetin aktiviert wird. Diese Aktivierung fördert die Abschreibung von Genen die zur Synthese von Enzymen, welche ROS neutralisieren, beitragen und daher auch ROS-induzierte Zellschäden reduzieren. Quercetin verringert auch die Freisetzung von Entzündungsmediatoren in den Zellen und im Blut von IPF-Patienten. Kapitel 4 beschreibt den Effekt von Quercetin in einem Bleomycin-induzierten Fibrose-Modell in Mäusen. Eine Woche nach der Quercetin-Einnahme durch die Nahrung erhöht sich die Quercetinkonzentration im Plasma, nicht aber in der Lunge, und die pulmonale Expression von Nrf2-abhängigen Genen erhöht sich (Kapitel 4). In wildtype Nrf2 Quercetingefütterten Mäusen zeigt sich eine verminderte Expression von Bindegewebeproteinen und es verringert entzündliche Läsionen in der Lunge. Zudem reduziert Quercetin die pulmonale Genexpression der Entzündungsproteine TNF $\alpha$ und KC, nicht jedoch deren Plasmakonzentration, und reduziert Malondialdehyd-dG-DNA-Addukte (oxidative DNASchädigung). Bei Mäusen ohne funktionierendem Nrf2 wird bei Einwirkung von Bleomycin kein positiver Effekt von Quercetin auf die entzündlichen Lungenläsionen beobachtet, was darauf schließen lässt, dass Quercetin durch die Induktion von Nrf2 vor Bleomycininduzierten Lungenschäden schützt. Die Quercetin-Supplementierung kann jedoch die durch Bleomycin verursachten Schäden nicht erheblich reduzieren, was darauf hindeutet, dass Quercetin nicht ausreicht, um die Entwicklung einer Lungenfibrose zu verhindern.

Die NADPH-Oxidase NOX4 ist eine wichtige Quelle für reaktive Sauerstoffradikale in der Lunge. Unter den NADPH-Oxidasen ist NOX4 einzigartig, da diese konstitutiv aktiv ist und Wasserstoffperoxid $\left(\mathrm{H}_{2} \mathrm{O}_{2}\right)$ produziert. NOX4 hat einen großen Einfluss auf eine Vielzahl von zellulären Prozessen und kann die Aktivität von Proteinen durch deren Oxidation beeinflussen. Jedoch sind Proteine, die durch NOX4 modifiziert werden können, weitgehend unbekannt. In Kapitel 5 wird erläutert, dass NOX4 das SRC-Kinase Mitglied FYN oxidiert und dadurch aktiviert in humanen bronchialen Epithelzellen. Die SRC-Familie der Tyrosinkinasen sind Proteine, deren Aktivität hauptsächlich durch Phosphorylierung und Dephosphorylierung reguliert werden. Diese Aktivierung von SRC-Kinasen spielt eine wichtige Rolle in der intrazellulären Signaltransduktion. Wissenschaftler haben 
herausgefunden, dass die Deaktivierung von SRC-Kinasen eine wichtige Rolle in der Entwicklung von Lungenfibrose spielt, es ist jedoch nicht bekannt wie sie dazu beitragen. Wenn FYN inhibiert wird, reduziert es die ROS-Produktion durch die Mitochondrien, was auch zu einer Verringerung von oxidativem DNA-Schaden und Expression von Bindegewebeproteinen führt. Der SRC-Inhibitor Saracatinib verringert die Aktivierung von Fibroblasten, die ROS-Produktion sowie die Freisetzung von Entzündungsmediatoren, wie in Kapitel 6 dargestellt wird. Interessanterweise aktiviert TGF- $\beta 1$ das SFK-Mitglied YES in Fibroblasten, was zur Expression von Bindegewebeproteinen in Fibroblasten beiträgt. Darüber hinaus ist der TGF-induzierte Anstieg der NOX4-Expression in YES-negativen Zellen reduziert, was darauf hindeutet, dass die Aktivierung von YES in Fibroblasten potentiell für die Induktion von NOX4 verantwortlich ist.

In Kapitel 7 wird gezeigt, dass bronchiale Epithelzellen von IPF-Patienten eine erhöhte Aktivierung von SRC-Kinasen aufweisen. Die Hemmung dieser Kinasen mit dem Inhibitor Saracatinib, reduziert Markers der mitochondrialen Dysfunktion, indem die Expression von Genen verstärkt wird, die mit der mitochondrialen Biogenese und Mitophagie assoziiert sind. Darüber hinaus verringert die SFK-Hemmung die Freisetzung von LDH, was auf eine Zellschädigung hinweist, und die Expression des pro-apoptotischen Gens BAX sowie das ROS-produzierende Enzym NOX4. In differenzierten bronchialen Epithelzellen ist die Expression von NOX4 bei IPF-Patienten im Vergleich zu Epithelzellen von Kontroll-Patienten leicht erhöht, unterscheidet sich jedoch erheblich zwischen individuellen IPF-Patienten (Kapitel 8). Derzeit gibt es keine voll wirksamen Behandlungsmöglichkeiten für IPF. Es gibt zwei Medikamente, die der Verschlechterung der Erkrankung entgegenwirken: Pirfenidon und Nintedanib. Diese wirken jedoch nicht bei allen Patienten. Interessanterweise induziert Pirfenidon bei Patienten mit höherer NOX4-Expression die Expression von antioxidanten Genen. Zusätzlich ist der Entzündungsmediator IL-8 in HBE-Zellen von IPF-Patienten signifikant erhöht und die Behandlung mit Saracatinib reduziert diese erhöhte IL-8-Sekretion. Nachdem die Wirkungen der zugelassenen Arzneimittel Pirfenidon und Nintedanib mit dem SFK-Inhibitor Saracatinib in differenzierten bronchialen Epithelzellen verglichen wurden, wird eine personalisierte Behandlung von IPF-Patienten empfohlen, da die Reaktion auf verschiedene Arzneimittel zwischen IPF Patienten sehr unterschiedlich ist und zusätzlich von ihrem Redox- und Entzündungsstatus abhängt.

Im letzten Kapitel dieser Arbeit (Kapitel 9) werden die wichtigsten Ergebnisse zusammengefasst und diskutiert. Zusammenfassend trägt diese Arbeit zum Verständnis der Bedeutung von Redoxregulation bei der Entstehung von Lungenfibrose bei. Die Ergebnisse zeigen, dass die Modulation von Redox-empfindlichen Proteinen, wie den SRC-Kinasen, neue Hinweise für die Behandlung von Patienten mit IPF liefern kann. Darüber hinaus deuten die Ergebnisse darauf hin, dass mehr Arbeit an der 
personalisierten Behandlung geleistet werden muss. Da IPF eine sehr heterogene Krankheit ist, werden neue Therapiemethoden benötigt, die speziell auf die Patienten angepasst werden. Um diesen nächsten Schritt zu erreichen, wird eine Stratifizierung von Patienten-Subgruppen und -Markern benötigt, um die Therapieergebnisse in diesen verschiedenen Gruppen besser vorherzusagen. Da Biomarker für solch eine heterogene Erkrankung nur schwer zu erhalten sind, besteht ein alternativer Ansatz darin, verschiedene Arzneimittel vor dem Behandlungsbeginn an bronchialen Epithelzellen von Patienten zu testen, um die individuelle Therapie für die Patienten zu verbessern und die hohe Sterblichkeit einzudämmen. 



\section{Valorization}


The incidence of age-related lung diseases, including idiopathic pulmonary fibrosis (IPF), lung cancer and chronic obstructive pulmonary disease, is increasing worldwide, warranting more treatment strategies to counteract the high morbidity and mortality associated with these diseases. The work presented in this thesis focused on different redox-modulatory strategies to alleviate features of the age-related disease pulmonary fibrosis.

The next pages will provide an overview of the valorization of the research described in this thesis. Knowledge valorization is "the process of creating value from knowledge by making knowledge suitable and/or societal useful and by translating that knowledge into products, services, processes and entrepreneurial activity" (1). The valorization potential of the work described in this thesis will be discussed with focus on social and economic relevance, the implications for specific target groups outside of academia and possible innovations that can be created from this research as well as their potential implantation.

\section{Social and economic relevance}

Since the research performed in this thesis aimed at identifying novel pathways involved in the development of pulmonary fibrosis, the relevance and impact for the society may not be seen immediately but rather in the near future.

During the last years, the average life expectancy increased worldwide. It is estimated that the number of people over 60 years of age will increase by almost $50 \%$ between 2015 and 2050 , thereby accounting for $22 \%$ of the total world population (2). Due to this increase, it is expected that the incidence of age-related lung diseases such as IPF will also increase at a rapid pace. However, the incidence of IPF is also increasing because of better options for diagnosis as well as the increased social awareness. The disease develops asymptomatically for months to years and normally the disease is diagnosed when the patient has already problems breathing which means that there is already significant damage to the lung tissue. Therefore, the survival of patients with IPF after diagnosis is estimated to be only 2-4 years (3). A diagnosis of IPF requires the exclusion of other forms of interstitial lung diseases such asbestos- or bleomycin-induced pulmonary fibrosis since the cause of the disease is not clear, leading to the diagnosis idiopathic pulmonary fibrosis. Therefore, no strategies for disease prevention can be developed.

Currently, there are approximately at least 5 million patient with IPF worldwide but studies have already suggested that the prevalence of IPF is increasing every year by $11 \%$ (4). Therefore, the socioeconomic burden related to IPF will also increase in the nearby feature. It has been indicated that once a patient is diagnosed with IPF, the health costs increase from approximately $€ 2.700$ to $€ 21.000$ per year without the cost of antifibrotic treatment (5). These costs derive from the fact that patients with IPF are often hospitalized also due to comorbidities such as pulmonary arterial hypertension, heart failure, pneumonia and lung cancer. Patients with IPF are admitted twice as often to the 
hospital compared to aged matched controls associated with an average cost per IPFrelated hospitalization of $\$ 16.812(6)$. Additionally, patients with IPF need continuous oxygen supply to increase the low levels of oxygen in the blood in a more severe stage due to the significant lung damage.

The impact of IPF is not only limited to a decline in lung function but is also strongly associated with a decrease in health associated quality of life because of the daily limitations in regard to everyday physical and social activities.

Although IPF is such a devastating disease, there are currently only two FDA-approved therapies available for its treatment which will not cure the already induced pulmonary damage. Pirfenidone and nintedanib each cost approximately $\$ 100.000$ per patient per year in the US (7) and they are not equally effective in all patients. The other costly therapy option is lung transplantation, which is not available for every individual patient. Consequently, there is an urgent need to perform more studies to identify novel leads for the development of new treatment options for pulmonary fibrosis. Our studies have indicated that the oxidant producing enzyme NOX4 oxidizes members of the SRC family kinases, thereby promoting features of pulmonary fibrosis. These observations suggest that targeting redox-sensitive proteins might be a lead for the treatment of pulmonary fibrosis. Moreover, our findings suggest that inhibition of SRC family kinases by their pharmacological inhibitor saracatinib might provide an alternative approach to reduce the progression of IPF.

Every individual is different in terms of their physiological and genetic makeup, which can also be deducted from our studies that show that the responsiveness to different drugs varies in epithelial cells derived from patients with IPF. Furthermore, these results have suggested that not all patient with IPF suffer from an increased oxidant burden, indicating even more the need to implement personalized treatment strategies to maximize the treatment benefits. One way of achieving personalized treatment could include the identification of lung damage biomarkers which would also improve diagnostics as IPF is usually diagnosed when it is already too late, and the lung is already severely damaged. Another way of achieving a more personalized treatment approach, could be testing individual responses to treatments in pulmonary epithelial cells of IPF patients isolated by non-invasive bronchoscopic microsampling directly upon diagnosis. In that way, treatment responses can be predicted on forehand, thereby personalizing and thus optimizing treatment. By using such an approach, costs can also be reduced as the treatment response is evaluated beforehand so that the patient does not have to be switched to a different drug in case of non-responsiveness. Altogether, the studies presented in this thesis may give rise to new therapy strategies to decrease the economic and health care burden associated with IPF. 


\section{Target groups}

The findings presented in this thesis are especially of interest to the academic community as well as the pharmaceutical industry. The results described in this thesis have been presented at various national and international meetings to discuss them with peers inside the research field. For instance, the work presented in this thesis has been presented at the annual meetings of the European Respiratory Society and the ERS Lung Science Conference as well as at the NOX NADPH Oxidases Gordon meetings. Moreover, the results described in this thesis are published or will be published in peer-reviewed journals to make it available to the community and to increase knowledge transfer.

However, it is also of great importance to present scientific work outside the field to the general public to increase social awareness of IPF. Scientific research is the basis for all progress and without research, treatment developments stand still. Research gives lung patients the prospect of improvement of their health status. Within the scientific community, we have teamed up with a specialized lung hospital to work with primary human lung cells directly derived from IPF patients. Part of this work was sponsored by the Longfonds which is a Dutch organization that promotes the communication form the lab bench to the general community and in specific to patients with a lung disease. Consequently, some results presented in chapter 7 and 8 have also been published in the newspaper and the homepage of this organization, making them accessible for everyone.

During the last decade, the pharmaceutical industry has become very interested in studying rare diseases and as a result, various new rare-disease treatments have entered the market. Since there are only two drugs available for IPF treatment and novel therapeutic interventions are necessary, it is a highly profitable industry. It has been suggested that the IPF drug market will increase from $\$ 900$ million in 2015 to $\$ 3,2$ billion by 2025 (8). Additionally, drugs that have the possibility to attain orphan drug status (when only a small percentage of the population is affected by the disease) are very attractive for the pharmaceutical industry as the status implies support for clinical trials, reduced fees as well as a 10-year patent protection to motivate the pharmaceutical companied to invest in these drugs. Currently, various IPF drugs are being evaluated in clinical trials (9) and our data also suggest that the SFK inhibition by saractinib would be a possible treatment option for IPF patients and would therefore be of interest to the pharmaceutical industry. Intriguingly, saracatinib has just received orphan drug status by the FDA in the US (March 2019) (10).

\section{Innovation}

Our understanding regarding the role of ROS in IPF is still very limited mainly due to the fact that detection of oxidant pathways in clinical specimens is difficult and methods to analyze them are limited. Especially reversible modifications of redox-sensitive proteins 
may represent an important mechanism in dysregulated cell signaling and might be more important than stable protein oxidation products. However, studies are very limited. Another problem with studies of ROS in disease models such as IPF is that they typically address the importance of a specific NOX enzyme or antioxidant system throughout the disease model, which may not necessarily translate into clinical practice where different pathways are involved. Consequently, it might be more important to address specific modifications of proteins as we did in chapter 5 , where we focused on one specific cysteine oxidation which might be a novel mechanism of SRC kinase activation.

Addressing the causality of specific ROS pathways requires the use of appropriate models, and currently used models of pulmonary fibrosis do not adequately model the pathophysiology of human IPF and its progression. In our studies, we have used different models (cell lines, bleomycin mouse model and primary cells derived from IPF patients) to study the effects of oxidant regulation in IPF development. Moreover, we have also utilized different cell types, i.e. epithelial cells and fibroblasts, to study the effects of SRC family kinase inhibition. It is of great importance to not only address treatment responses in fibroblasts, the effector cells, but also to investigate them in epithelial cells, which initiate and mediate fibrotic and inflammatory responses. The lung epithelium plays a crucial role in the development of IPF which makes it pivotal to investigate the effects of fibrotic drugs on the lung epithelium as well. Also, while IPF is well-known to be ageassociated, most studies are still performed only in cell lines which does not recapitulate the contribution of age-associated alterations. Therefore, we have also investigated the effects of SRC family kinase inhibition on primary epithelial cells isolated from patients with IPF. Although our knowledge about IPF is growing, we still know relatively little with respect to the precise molecular mechanisms. The results in this thesis contribute to our understanding regarding the effects of oxidant regulation and targeting of redox-sensitive proteins in the pathophysiology of IPF. However, to effectively treat every individual patient, better treatment options need to be developed and if possible individualized, also called personalized medicine.

\section{Planning and implantation}

Before personalized medicine with respect to IPF can be implemented into the clinic, more studies need to be performed on the use of bronchial epithelial cells isolated by bronchoscopic microsampling to predict therapy outcome. Based on our preliminary pilot data, it can be suggested that patients could be divided in subgroups possibly based on their redox or inflammatory status or the activity of SRC kinases. In the beginning, dividing patients into specific subgroups will increase the associated treatment costs but, in the future, this will decrease costs associated with false treatments in addition to increasing treatment effectiveness. This thesis provides a first lead to study the importance of the redox-sensitive proteins SRC kinases and their modifications by the oxidant producing 
enzyme NOX4 and subsequently investigated the effects of their inhibition. Additionally, this thesis provides first ideas to implement a more personalized approach in the treatment of pulmonary fibrosis. 


\section{References}

1. Rathenau Instituut. Valorisation: researchers already do much more than they realise 2016 [cited $20194 \mathrm{Mar}$. Available from: https://www.rathenau.nl/en/knowledge-policy/valorisationresearchers-already-do-much-more-they-realise.

2. World Health Organization. Ageing and health 2018 [cited 201826 Nov]. Available from: https://www.who.int/news-room/fact-sheets/detail/ageing-and-health.

3. Raghu G, Collard HR, Egan JJ, Martinez FJ, Behr J, Brown KK, et al. An official ATS/ERS/JRS/ALAT statement: idiopathic pulmonary fibrosis: evidence-based guidelines for diagnosis and management. Am J Respir Crit Care Med. 2011;183(6):788-824.

4. Gribbin J, Hubbard RB, Le Jeune I, Smith CJ, West J, Tata LJ. Incidence and mortality of idiopathic pulmonary fibrosis and sarcoidosis in the UK. Thorax. 2006;61(11):980-5.

5. Hilberg O, Bendstrup E, Ibsen R, Lokke A, Hyldgaard C. Economic consequences of idiopathic pulmonary fibrosis in Denmark. ERJ Open Res. 2018;4(2).

6. Yu YF, Wu N, Chuang CC, Wang R, Pan X, Benjamin NN, et al. Patterns and Economic Burden of Hospitalizations and Exacerbations Among Patients Diagnosed with Idiopathic Pulmonary Fibrosis. J Manag Care Spec Pharm. 2016;22(4):414-23.

7. Owens GM. Strategies to manage costs in idiopathic pulmonary fibrosis. Am J Manag Care. 2017;23(11 Suppl):S191-S6.

8. White V. IPF market will grow to $\$ 3.2$ billion by 2025 , says GlobalData: European Pharmaceutical Review; 2016 [cited 201912 Apr]. Available from:

https://www.europeanpharmaceuticalreview.com/news/41884/ipf-market-globaldata/.

9. Mora AL, Rojas M, Pardo A, Selman M. Emerging therapies for idiopathic pulmonary fibrosis, a progressive age-related disease. Nat Rev Drug Discov. 2017;16(11):755-72.

10. AstraZeneca. US FDA grants saracatinib Orphan Drug Designation for idiopathic pulmonary fibrosis 2019 [cited 201912 Apr]. Available from: https://www.astrazeneca.com/mediacentre/press-releases/2019/us-fda-grants-saracatinib-orphan-drug-designation-for-idiopathicpulmonary-fibrosis-18032019.html. 

Acknowledgements

\author{
Dankwoord
}

Danksagungen 
This acknowledgement section is the last piece of the puzzle and the most difficult one to write. Probably also because it is the end of a very important chapter in my life. During the last years I had to face disappointments and frustration combined with a lot of happy moments. It is impossible to identify every single person that has helped me through this time, but a lot of wonderful people played crucial roles along the way. I feel it only appropriate to address them in random chronological order and will already apologize for jumping between languages.

First of all, a big Dankeschön to the people I probably drove crazy during the last three months of thesis writing, I couldn't have wished for more supportive friends and colleagues!!!

Mama und Papa, danke, dass ich mich immer unterstützt habt egal was für eine verrückte Idee ich hatte wie als ich von Bühl in die Niederlande oder von den Niederlanden nach Amerika bin. Ich habe von euch nie die Worte „du kannst das nicht oder du schaffst das nicht" gehört. Ohne euch wäre das alles nie möglich gewesen! Anja, du hast mich immer unterstützt egal was für eine verrückte Idee mir mal wieder im Kopf rumschwirrt oder mich auf den Boden der Tatsachen zurück geholt. Danke dass ich dich ab und zu in den Wahnsinn treiben darf!

To my girls: Kügele alias Jasmin, was hätte ich nur ohne dich getan. Du bist immer da egal was für ein Tag oder Uhrzeit es ist um dich von mir voll heulen zu lassen. Ohne deine Unterstützung wären die letzten Jahren kaum möglich gewesen! Tanja, danke dass du immer ein offenes Ohr hast und dir immer Zeit für uns nimmst wenn ich mal in der Nähe bin. Birgit, du bist nicht nur ein unglaublich kreatives Talent, sondern auch eine ganz wunderbare Person mit der ich über alles quasseln kann. Danke euch für die unzähligen Erlebnisse und Momente die wir schon zusammen geteilt haben. Es ist unschätzbar wertvoll für mich, dass wir nach so vielen Jahren immer noch füreinander da sind. Franz von Assisi sagte einmal „Alte Freunde sind wie alter Wein, er wird immer besser, und je älter man wird, desto mehr lernt man dieses unendliche Gut zu schätzen“.

To the friends that are family: Johanna, this year we already celebrate our 10-year anniversary! Eine Million gemeinsame Erlebnisse, tausend peinliche Bilder und zahlreiche Insider Jokes weiter, sagst du mir immernoch die knallharte Wahrheit. Thank you for always being there and for your critical input on big scientific and life decisions! En de rest van de MLW-crew: Niels, Camiel, Joël, Max, Claudia, Alex, Svenja, Thomas, Leslie, Mandy en Mick, bedankt voor de gezellige weekenden, festivals, borrels en feestjes. Bedankt voor jullie ondersteuning en de ontzettend leuke momenten! 
To my Vermont Family: Milena, a big thanks for all the support in the lab and for all your general input ranging from which jeans to buy to which pipet to use! To the UVM crew: Matt, Dave, Andrew, Chris, Caspar and Cheryl, I had an awesome time in Burlington, also outside the lab, because of you!

To all the (Phar)TOX (Ex)PhD-Colleagues: Kristien, Erik, Agnieszka, Mireille, Gesiele, Kevin, Quan, Kim, Sven, Christy, George, Wenbo, Ming, Ellen, Stefan, Takashi, Shan, thank you for all the help, discussions, encouraging and kind words, and of course all the fun we had! Misha, thank you for being my roommate and always listening to me! Philippe, bedankt voor al de gezellige avonden op het lab, met jou was het nooit saai. Ik zal onze lange babbeltjes missen! In het bijzonder, Robert, bedankt voor de korte maar gezellige tijd op ons kantoor. De relax momentjes waren de highlights van mijn dag tijdens deze drukke tijd! Charlotte, Rianne en Alie, bedankt voor jullie kritische input en al de leuke momenten die wij samen hadden en nog zullen hebben! I can always count on you!

In het bijzonder, Timme, we hadden meteen een klik, en ik ben blij dat we meer zijn dan alleen maar collega's. Bedankt voor je vriendschap, ook over een oceaan heen, kon ik altijd bij jou terecht. I already miss your face (en ook de rest)! Ik ben super blij dat je mijn paranymphe bent, en achter mij staat tijdens dit belangrijk moment!

En de hele afdeling Pharmacology and Toxicology, bedankt voor al jullie hulp tijdens de laatste jaren, voor de leuke activiteiten en natuurlijk de borrels. Het was altijd gezellig!

Lou en Marie-José in het bijzonder, bedankt voor all jullie hulp. Vooraal tijdens de laatste maanden, als ik het soms niet meer zag zitten en jullie altijd een hand (en oor of soms ook beide) vrij hadden voor mij.

Eddie, wat had ik soms zonder jou gedaan, als ik iets had besteld en snel nodig had, kwam je het meteen brengen. Bedankt voor de gezellige gespreken en je happy face. Alex en Will, bedankt voor al de gezellige tijden op de vrijdag middag en jullie heerlijke humor.

Marie-Claire, bedankt voor al je hulp en waardevolle gespreken tijdens de laatste jaren! Als ik soms niet wist wat ik moest doen, was je er altijd om te luisteren en advies te geven.

Thank you, Agnes, for your guidance, patience, mentorship, all the coffee, and all the discussions we had about science and life. Thank you for always looking out for me and calming me down when I was getting too obsessed about small stuff. I will always remember our long email conversations before big deadlines, I couldn't have imagined a more supportive supervisor! 
Frederik-Jan, you have given me freedom in my projects and were always there to discuss new exciting results and always had new input. You always kept your sense of humor especially in times when I lost mine. Thank you for believing in me!

Albert, thank you for introducing me to the world of NOXes during my Masters. When I first arrived in your lab in 2013, I wasn't even sure if I wanted to become a scientist. You have taught me more than I could have imagined and your enthusiasm and dedication to science is more than inspiring. Thank you for being a great mentor even when I wasn't in your lab. Thank you for your patience, motivation, guidance and perfectionism.

I am immensely grateful, that I could give my research the translational link through the collaboration with the Thoraxklinik Heidelberg. Thank you, Nicolas, for giving me the chance to work with these precious cells from IPF patients and all your input on the grant proposals! We have some great preliminary results and these studies turned into great chapters in my thesis! Liz, thank you for all your help in the lab and critical input on the setup of all the experiments, without you it wouldn't have been possible! Marc, thank you for your time and helping me with the imaging experiments!

Last but the least aan de leden van de leescomissie: Annemie Schols, Matthijs Blankesteijn, Wilfred Germeraad, Jan Grutters en Pieter Hiemstra, bedankt dat jullie de tijd hebben genomen om mijn proefschrift door te lezen en te beoordelen.

The last years went by so fast and sometimes so slow at the same time. I am ultimately grateful that I have met so many wonderful people during the last 4.5 years in 3 different countries, which makes the world feel a bit smaller: "How lucky I am to have something that makes saying Goodbye so hard" (Winnie the Pooh). 
List of publications 


\section{Published/ accepted for publication}

Veith C, Boots AW, Idris M, van Schooten FJ, van der Vliet A. Oxidant crosstalk in IPF - Are NADPH oxidases involved in mitochondrial dysfunction? Antioxidant Redox signaling. 2019, Epub Ahead of Print (doi: 10.1089/ars.2019.7742).

Veith C, Drent M, Bast A, Schooten FJ, Boots AW. The disturbed redox-balance in pulmonary fibrosis is modulated by the plant flavonoid quercetin. Toxicology and Applied Pharmacology. 2017; 336: p. 40-48.

Shi Q, Boots AW, Maas L, Veith C, van Kuijk K, Haenen GR, Godschalk RW, Van Schooten FJ. Effect of interleukin (IL)-8 on benzo[a]pyrene metabolism and DNA damage in human lung epithelial cells. Toxicology. 2017; 381: p. 64-74.

Shi Q, Maas L, Veith C, Van Schooten FJ, Godschalk RW. Acidic cellular microenvironment modifies carcinogen-induced DNA damage and repair. Archives of toxicology. 2017; 91(6): p. 2425-2441.

Hristova M, Habibovic A, Veith C, Janssen-Heininger YM, Dixon AE, Geiszt $M$, van der Vliet A. Airway epithelial dual oxidase 1 mediates allergen-induced IL-33 secretion and activation of type 2 immune responses. The Journal of allergy and clinical immunology. 2016; 137(5): p. 1545-1556.

Shi Q, Haenen GR, Maas L, Arlt VM, Spina D, Vasquez YR, Moonen E, Veith C, Van Schooten FJ, Godschalk RW. Inflammation-associated extracellular beta-glucuronidase alters cellular responses to the chemical carcinogen benzo[a]pyrene. Archives of toxicology. 2016; 90(9): p. 2261-73.

Hristova M, Veith C, Habibovic A, Lam YW, Deng B, Geiszt M, Janssen-Heininger YM, van der Vliet A. Identification of DUOX1-dependent redox signaling through protein Sglutathionylation in airway epithelial cells. Redox biology. 2014; 2: p. 436-46. 


\section{Submitted/ in preparation}

Veith C*, Boots AW*, Albrecht CA, Bartholome R, Drittij MJ, Claessen SMH, Bast A, Rosenbruch $\mathrm{M}$, Jonkers K, van Schooten FJ, Schins RPE. The dietary antioxidant quercetin reduces hallmarks of bleomycin-induced fibrogenesis in mice. Submitted.

Veith C, Hristova M, Danyal K, Habibovic A, van Schooten FJ, Boots AW, van der Vliet A. Profibrotic signaling by TGF- $\beta 1$ involved NADPH oxidase 4 dependent activation of the tyrosine kinase FYN. Submitted.

Veith C, Boots AW, Drittij MJ, Kreuter M, Schneider M, Meister M, Schooten FJ, Kahn N. Differences in treatment responses in bronchial epithelial cells from Idiopathic pulmonary fibrosis patients: a first step towards personalized medicine? In preparation.

Veith C, Kahn N, Remels A, Maas L, Kreuter M, Schneider M, Meister M, van $\operatorname{der}$ Vliet A, van Schooten FJ, Boots AW. Inhibition of SRC family kinases alleviates impaired expression levels of key regulators of mitochondrial biogenesis and mitophagy in bronchial epithelial cells of IPF patients. In preparation.

Veith C, Idris M, Drittij MJ, van der Vliet A, van Schooten FJ, Boots AW. SRC family kinases are mediating fibroblast activation through TGF- $\beta 1$ induced activation of NOX4. In preparation. 


\section{Conference abstracts}

Veith C, Kahn N, Maas L, van der Vliet A, van Schooten FJ, Kreuter M, Schneider M, Meister $M$, Boots AW. SRC family kinase inhibition reduces NOX4 expression in primary bronchial epithelial cells form IPF patients. Annual meeting DZL 2019, Mannheim, Germany (Poster presentation).

Veith C, Hristova M, Danyal K, Habibovic A, van Schooten FJ, Boots AW, van der Vliet A. Profibrotic signaling by TGF- $\beta 1$ involved NADPH oxidase 4 dependent activation of the tyrosine kinase FYN. Gordon Research seminar and conference NOX family NADPH oxidases 2018, Les Diablerets, Switzerland (oral presentation and poster presentation).

Veith C, Hristova M, van Schooten FJ, Boots AW, van der Vliet A. Profibrotic signaling by TGF- $\beta 1$ involves NADPH oxidase 4 dependent activation of tyrosine kinase SRC and mitochondrial ROS. ERS Lung Science Conference 2017, Estoril, Portugal (Poster presentation).

Veith C, van Schooten FJ, van der Vliet A, Maas K, Boots AW. NADPH oxidases induce oxidative damage in an in vitro model of pulmonary fibrosis. Gordon Research seminar and conference NOX family NADPH oxidases 2016, Waterville Valley, NH, USA (oral presentation and poster presentation)

Veith C, van Schooten FJ, Maas L, Boots AW. The role of Nox4 in the induction of profibrotic effects by TGF- $\beta 1$ in vitro. Annual Meeting Nutrim Research School 2015, Maastricht, NL (Poster presentation).

Veith C, van Schooten FJ, Jonkers L, Schins R, Albrecht C, Boots AW. Bleomycin-induced pro-fibrotic lung damage is accompanied by genotoxicity. Annual Meeting Nutrim Research School 2014, Maastricht, NL (Poster presentation).

Veith C, Jonkers L, van Schooten FJ, Boots AW. In vitro modulation of the disturbed redoxbalance in pulmonary fibrosis by quercetin. Annual Meeting Dutch Society for Toxicology (NVT) 2014, Veldhoven, NL. 


Curriculum Vitae 


\section{About the author}

Carmen Veith was born on the $3^{\text {rd }}$ of February 1990 in Buehl, Germany. After finishing high school in 2009, she studied Molecular Life Sciences at Maastricht University. After graduating in 2009, she continued with the Master program Biomedical Sciences with specialization Clinical Molecular Life Sciences. During her Master, she worked on redox-dependent mechanisms in the pathophysiology of asthma at the University of Vermont. For her Master thesis she studied the effects of the antioxidant quercetin

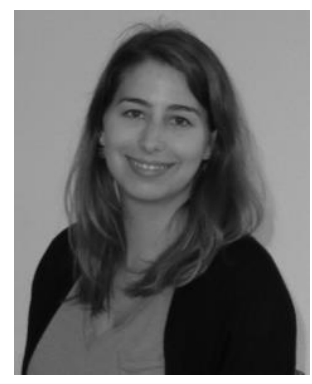
in the context of pulmonary fibrosis at Maastricht University which was awarded with the Unilever Research Prize in 2014.

After graduation in 2014, she was awarded a Kootstra and Nutrim GP grant which allowed her to start as a PhD student at the department of Pharmacology \& Toxicology at Maastricht University in collaboration with the department of Pathology and Laboratory Medicine at the University of Vermont. From September 2014 until February 2019, she worked on the role of redox regulation in the development of pulmonary fibrosis and conducted the studies presented in this thesis under supervision of Prof Dr. Frederik-Jan van Schooten, Prof. Dr. Albert van der Vliet and Dr. Agnes Boots. She worked at the University of Vermont to study NOX4-dependent redox mechanisms and investigated the effects of different antifibrotic drugs on lung cells from pulmonary fibrosis patients at the Thoraxklinik University hospital Heidelberg, which was funded by grants from the Longfonds and the European Respiratory Society (ERS). She attended several courses and conferences and was awarded several prizes and grants during that period. After finishing her PhD, she started working as a Postdoctoral Fellow at the German Cancer Research Center to further study NOX enzymes and their interaction with receptor tyrosine kinases. 